\title{
PROCEEDINGS
}

OF THE

American Mathematical Society

\author{
EDITED BY \\ FRED G. BRAUER \\ ERNEST A. MICHAEL \\ W. H. J. FUCHS \\ JOSEPH J. ROTMAN \\ IRVING GLICKSBERG \\ GEORGE B. SELIGMAN \\ EMERY THOMAS
}

WITH THE COOPERATION OF

Joshua Chover

Allen L. Shields

Robert I. Soare

VOLUME 30, NUMBER 4

DECEMBER, 1971

CODEN: PAMYAR

PUBLISHED BY THE AMERICAN MATHEMATICAL SOCIETY PROVIDENCE, RHODE ISLAND 


\section{Proceedings of the American Mathematical Society}

This Journal is devoted entirely to research in pure and applied mathematics, and the publication of original papers of moderate length. The maximum length of an acceptable paper is about 8 printed pages. Since a page of the PrOCEEDINGs contains about 400 words, a rule of thumb is that under 10 typed pages is probably within the limit, but that over 12 typed pages is probably too long.

SHORTER NOTES. Very short notes not to exceed 1 printed page of an unusual nature are also accepted, and appear under the heading SHORTER NOTES. Items deemed suitable include an elegant new proof of an important and well-known theorem, an illuminating example or counterexample, or a new viewpoint on familiar results. New results, if of a brief and striking character, might also be acceptable, though in general a paper which is merely very short will not be suitable for the SHORTER NOTES department.

Preparation of THe MANUSCRIPT. Articles for insertion should be typewritten and double spaced. Ditto is not generally satisfactory, although other modes of multiple reproduction may be. The Manual for Authors, available from the Society, should be consulted for symbols and style conventions. Authors should take the greatest possible care in preparing the original manuscript. Hand drawn symbols are satisfactory, if clearly done; directions to the printer should be included where necessary on a separate sheet, not in the accompanying letter. Authors must keep a complete copy of their manuscript, and editors will acknowledge receipt; manuscripts can therefore be sent by ordinary mail and any other kind (registered, certified) is entirely unnecessary. Submission of two copies of the manuscript is helpful, but by no means necessary.

FORM OF MANUSCRIPT. The first page should consist of a descriptive title, followed by an abstract which summarizes the article in language suitable for workers in the general field (algebra, analysis, etc.). The descriptive title should be short, but informative; useless or vague phrases such as "some remarks about" or "concerning" should be avoided. Also avoid proper names unless mathematical usage associates them with the work. The abstract should be at least one complete sentence, and at most 150 words. Included with the footnotes to your paper, but placed before the first footnote, there should be first the AMS (MOS) subject classification numbers representing the primary and secondary subjects of the article. This may be followed by a list of key words and phrases describing the subject matter of the article and taken from it. The AMS (MOS) Subject Classification Scheme (1970) with instructions for its use can be found as an appendix to Mathematical Reviews, Index to Volume 39 (June 1970). See the June 1970 Notices for more details, as well as illustrative examples.

Submission of manuscripts, Reprints and AdDress Changes. See the last page of this issue.

SubSCRIPTION INFORMATION. Four volumes are planned for 1971. The subscription price is $\$ 72$. Back issues of volumes $1-16$ are available at a price of $\$ 14$ each. Volumes $17-19$ at a price of $\$ 18$ each, and Volumes 20 -30 at a price of $\$ 30$ each.

The Proceedings of the American Mathematical Society is published monthly. Subscriptions, orders for back numbers and inquiries in regard to nondelivery of current numbers should be addressed to the American Mathematical Society, P.O. Box 6248, Providence, R.I. 02904.

Second-class postage paid at Providence, Rhode Island, and additional mailing offices. 


\section{PROCEEDINGS \\ OF THE \\ AMERICAN MATHEMATICAL SOCIETY}

INDEX

VOLUMES 21-30

April 1969 - December 1971

\section{CONTENTS}

\begin{tabular}{|c|c|}
\hline Editorial Staff & $\begin{array}{l}\text { Page } \\
616\end{array}$ \\
\hline Author Index & 617 \\
\hline Subject Index & .713 \\
\hline
\end{tabular}




\section{PROCEEDINGS EDITORIAL COMMITTEE}

\section{April 1969-December 1971}

Fred G. Brauer, 1969-1971

W. H. J. FuCHS, 1969-1971

IRVING GLICKSBERG, 1969-1971

ARTHUR MATTUCK, 1969-1970

ERNeST A. Michael, 1969-1971
IRVING REINER, 1969

JOSEPH J. ROTMAN, 1970-1971

George B. Seligman, 1970-1971

EMERY THOMAS, 1969-1971

WolfGang R. Wasow, 1969

\section{ASSOCIATE EDITORS}

W. W. BOONE, 1969-1971

Joshua Chover, 1969-1971

S. M. SHAH, 1969

Allen L. Shields, 1970-1971

ROBERT I. SOARE, 1971

HANS Weinberger, 1969

This 10-VOLUME INDEX includes both an author and a subject listing. In the author index each entry gives complete information including title, volume, page, and subject classifications; the AMS(MOS) subject classification scheme (1970) is used. Primary subject classification numbers are given for all articles; if there are secondary subject classifications, they are separated from the primary classifications by a semicolon.

In the subject index the papers are identified by author's name and sequence number. Each entry is followed by a $P$ or an $S$, indicating whether the subject classification is Primary or Secondary. 


\section{AUTHOR INDEX}

Aarnes, Johan F.

1 (with Kadison, Richard V.) Pure states and approximate identities, 21 (1969), 749-752.

Aberth, Oliver

1 A chain of inclusion relations in computable analysis, 22 (1969), 539-548.

$02 \mathrm{E} 15$

2 The failure in computable analysis of a classical existence theorem for differential $\begin{array}{ll}\text { equations, } 30 \text { (1971), 151-156. } & 02 \mathrm{E} 15 ; 26 \mathrm{A03}\end{array}$

Abian, Alexander

1 Direct product decomposition of commutative semisimple rings, 24 (1970), 502-507.

13A99; 06A40 06A70 13A10

Abramowich, John

1 Stability of solutions of linear systems with retarded arguments, 26 (1970), 60-64.

Accola, Robert D. M.

$34 \mathrm{~K} 20$

1 Riemann surfaces with automorphism groups admitting partitions, 21 (1969), 477 482.

30A48 30A58

2 Two theorems on Riemann surfaces with noncyclic automorphism groups, 25 (1970), 598-602.

$30 \mathrm{~A} 48$

3 Strongly branched coverings of closed Riemann surfaces, 26 (1970), 315-322.

Adams, Robert A.

1 Equivalent norms for Sobolev spaces, 24 (1970), 63-66.

$12 \mathrm{~F} 10$

Adler, Andrew

1 Some recursively unsolvable problems in analysis, 22 (1969), 523-526.

$02 \mathrm{E} 9902 \mathrm{G} 05$

2 The cardinality of ultrapowers - An example, 28 (1971), 311-312.

Agoston, Max $\mathrm{K}$.

1 A classification of immersed knots, 24 (1970), 710-715. 57D40 57D60 57F20 Agrawal, Bhagwan Das

1 (with Khanna, I. K.) A general differential equation for classical polynomials, 22 (1969), 646-649. $\quad 33 A 4533 \mathrm{A65}$

Aizley, Paul

1 Multiplicative linear functionals on convolution algebras, 28 (1971), 65-66.

Akcoglu, M. A.

$10 \mathrm{~A} 20$

1 (with Cunsolo, J.) An ergodic theorem for semigroups, 24 (1970), 161-170.

2 (with Chacon, Rafael V. and Schwartzbauer, T.) Commuting transformations and $\begin{array}{ll}\text { mixing, } 24 \text { (1970), 637-642. } & \text { 28A65 }\end{array}$

Akemann, Charles A.

1 Separable representations of a $W^{*}$-algebra, 24 (1970), 354-355. 46L10 Al-Amiri, Hassoon S.

1 On p-close-to-star functions of order $\alpha, 29$ (1971), 103-108.

$30 \mathrm{~A} 32$

Alder, Henry $L$.

1 Proof of Andrew's conjecture on partition identities, 22 (1969), 688-689. 
Alexander, Charles C.

1 An extension of Morita's metrization theorem, 30 (1971), 578-582.

Alin, J. S.

54E35; 54D20 54E30

1 A primary decomposition for torsion modules, 27 (1971), 43-48.

Allan, G. R.

16A64; 16A46 16A62

1 A note on the holomorphic functional calculus in a Banach algebra, 22 (1969), 77-81.

\section{Allegretto, Walter}

46J05; 32B05

1 A generalization of Sturm's separation theorem, 25 (1970), 151-154.

35B05 $35 \mathrm{~J} 70$

2 (with Swanson, C. A.) Oscillation criteria for elliptic systems, 27 (1971), 325-330.

Allen, Paul J.

35B05 35J60

1 A fundamental theorem of homomorphisms for semirings, 21 (1969), 412-416.

Al-Salam, Waleed A.

$16 \mathrm{~A} 78$

1 (with Verma, Arun) Some orthogonality preserving operators, 23 (1969), 136-139.

Amberg, Bernhard

42A52; 34A35

1 (with Scott, W. R.) Products of Abelian subgroups, 26 (1970), 541-547.

$20 \mathrm{~F} 25$

Amoroso, $\mathbf{S}$.

1 (with Cooper, G.) The Garden-of-Eden theorem for finite configurations, 26 (1970), 158-164.

Andersen, G. $\mathbf{R}$.

05B30 68A25

1 Large deviation probabilities for positive random variables, 24 (1970), 382-384. 60F10 60G50; 26A12 60E05 62E20

Andersen, Tage Bai

1 On multipliers and order-bounded operators in $C^{*}$-algebras, 25 (1970), 896-899.

Anderson, Bruce A.

46L05; 47C10

1 (with Stewart, D. G.) $T_{1}$-complements of $T_{1}$ topologies, 23 (1969), 77-81.

54A10; 06A25

2 Families of mutually complementary topologies, 29 (1971), 362-368.

Anderson, R. D.

$54 \mathrm{~A} 05$ 54A 10

1 (with McCharen, John D.) On extending homeomorphisms to Fréchet manifolds, 25 (1970), 283-289.

58B05

Andrews, George E.

1 On Ramanujan's summation of ${ }_{1} \psi_{1}(a ; b ; z), 22$ (1969), 552-553. 33A30

2 On a transformation of bilateral series with applications, 25 (1970), 554-558.

$10 \mathrm{~J} 2033 \mathrm{~A} 30$

Antonelli, Peter L.

1 Montgomery-Samelson singular fiberings of spheres, 22 (1969), 247-250. 
Apostol, Tom M.

1 Resultants of cyclotomic polynomials, 24 (1970), 457-462. 12E10

2 Euler's $\phi$-function and separable Gauss sums, 24 (1970), 482-485. $\quad 10 \mathrm{G05}$ Appel, K. I.

1 On two variable equations in free groups, 21 (1969), 179-184. 20F10 Appling, William D. L.

1 Addendum to: Some integral characterizations of absolute continuity, 24 (1970), 788-793.

$28 \mathrm{~A} 10$

Arkowitz, Martin

1 Associative, abelian $H$-spaces have trivial Postnikov invariants, 25 (1970), 460-461.

55D45 55G45

Armacost, David Lee

1 Well-known LCA groups characterized by their closed subgroups, 25 (1970), 625-629.

20K45 22B05; 06A05

2 Can an LCA group be anti-self-dual, 27 (1971), 186-188. 22B05 43A40 Armendariz, Efraim P.

1 On finite-dimensional torsion-free modules and rings, 24 (1970), 566-571.

16A52; 16A12 16A46

Arnold, Jimmy T.

1 (with Gilmer, Robert W., Jr.) On the contents of polynomials, 24 (1970), 556-562.

13B25 13F20

2 (with Brewer, J. W.) Kronecker function rings and flat $D[X]$-modules, 27 (1971), 483-485.

13B25 13F05

Arora, Kasturi L.

1 (with Kulshreshtha, S. K.) An infinite integral involving Meijer G-function, 26 (1970), 121-125.

30A86 33A35 44A45; 33A15 33A30

Arsove, Maynard G.

1 A correction to 'Some boundary properties of the Riemann mapping function', 22 (1969), 711-712.

$30 \mathrm{~A} 30$

Asimow, Leonard

1 Decomposable compact convex sets and peak sets for function spaces, 25 (1970), 75-79.

$46 \mathrm{E} 15$ 46J20

Atalla, Robert E.

1 (with Bustoz, Joaquin) On sequential cores and a theorem of $R$. $R$. Phelps, 21 (1969), 36-42. 40C05 40H05 46A45 54A25 54C10 54C15 54E50; 46E10

2 An example in the Weil theory of measurable groups, 25 (1970), 816-819.

22A05 28A70; 22A10 43A05

3 On the multiplicative behavior of regular matrices, 26 (1970), 437-446.

40C05 47B99; 40D20 46E15 54D35

Athreya, Krishna Balasundaram

1 On the absolute continuity of the limit random variable in the supercritical GaltonWatson branching process, 30 (1971), 563-565.

Aull, C. E.

60E05 60J80 62E10; 39A15 60F15

1 Topological spaces with a $\sigma$-point finite base, 29 (1971), 411-416.

Auslander, Bernice

54D15 54D20 54E30 54E35 
1 Central separable algebras which are locally endomorphism rings of free modules, 30 (1971), 395-404.

13A20

Au-yeung, Yik-hoi

1 Some theorems on the real pencil and simultaneous diagonalization of two hermitian bilinear functions, 23 (1969), 246-253.

15A57 15A63

2 On matrices whose nontrivial real linear combinations are nonsingular, 29 (1971), 17-22.

15A30 15A57; 15A33

Bachelis, Gregory F.

1 On the ideal of unconditionally convergent Fourier series in $L_{p}(G), 27$ (1971), 309-312.

Bacon, Philip

43A15 43A20 43A50

1 Compact means in the plane, 22 (1969), 242-246.

Baggett, Larry

1 Hilbert-Schmidt representations of groups, 21 (1969), 502-506.

Bagley, Robert W.

22D10; 22D25 22E30

1 (with Weddington, D. D.) Products of $k^{\prime}$-spaces, 22 (1969), 392-394. 54D50

2 (with Lau, K. K.) Semidirect products of topological groups with equal uniformities, 29 (1971), 179-182.

22A05 22D05

Baisnab, A. P.

1 On a theorem of Goffman and Neugebauer, 23 (1969), 573-579.

Baker, J. M.

26A24 26A48

1 Weak sequential completeness in spaces of operators, 25 (1970), 193-198.

Baker, James D.

47D15; 46M05

1 (with Wright, Fred M.) On integration-by-parts for weighted integrals, 22 (1969), 42-52.

26A42

Baker, John Warren

1 (with Rothman, Neal J.) Separating points by semicharacters in topological semigroups, 21 (1969), 235-239.

22A15 22A20

2 Convolution measure algebras with involution, 27 (1971), 91-96.

Balbes, Raymond

43A10; 43A20

1 The center of the free product of distributive lattices, 29 (1971), 434-436.

Ballantine, Charles S.

06A35; 08A10

1 Stabilization by a diagonal matrix, 25 (1970), 728-734.

15A 18

Banchoff, Thomas F.

1 High codimensional 0-tight maps on spheres, 29 (1971), 133-137.

Bang, Chang Mo

53C40 53C45; 52A25 57C35 57D40

1 Direct sums of countably generated modules over complete discrete valuation rings, 28 (1971), 381-388.

13C05 16A64 20K25

Bank, Steven

1 On the growth of certain meromorphic solutions of arbitrary second order algebraic differential equations, 25 (1970), 791-797.

30A70 34A20 
Banks, Dallas O.

1 Inequalities for the eigenvalues of powers of functions, 23 (1969), 356-358.

Barnes, Bruce Alan

1 Irreducible algebras of operators which contain a minimal idempotent, 30 (1971), 337-342.

46L05 46L20

Barr, Michael

1 (with Knus, Max-Albert) Extensions of derivations, 28 (1971), 313-314.

1 On the existence of fundamental solutions of boundary problems, 24 (1970), 75-78.

\section{Barshay, Jacob}

1 A characterization of regular local rings, 29 (1971), 437-439. 13 H05 15A78 Barth, K. F.

1 (with Schneider, W. J.) An asymptotic analog of the F. and M. Riesz radial uniqueness theorem, 22 (1969), 53-54.

2 (with Schneider, W. J.) A short proof of a lemma of G. R. MacLane, 20(1969), 604-605.

Barth, Theodore J.

1 Taut and tight complex manifolds, 24 (1970), 429-431.

$32 \mathrm{C} 10$ Başgöze, Türkân

1 (with Keogh, F. R.) The Hardy class of a spiral-like function and its derivative, 26 (1970), 266-269.

30A30 30A32; 30A78

Basmaji, B. G.

1 On the isomorphisms of two metacyclic groups, 22 (1969), 175-182. 20D10 Bauman, Steven F.

1 A note on cover and avoidance properties in solvable groups, 21 (1969), 173-174.

Baumslag, Gilbert

20D10

1 (with Karrass, Abraham and Solitar, Donald) Torsion-free groups and amalgamated products, 24 (1970), 688-690.

$20 \mathrm{E} 30$

Baxter, J. R.

1 A class of ergodic transformations having simple spectrum, 27 (1971), 275-279.

28A65; 47A35

Beaumont, Ross A.

1 A note on products of homogeneous torsion free abelian groups, 22 (1969), 434-436.

Beauregard, Raymond A.

20K20

1 (with Johnson, R. E.) Primary factorization in a weak Bezout domain, 25 (1970), 662-665.

16A02; $13 \mathrm{G} 05$

2 Right LCM Domains, 30 (1971), 1-7.

16A02

Bebernes, J. W.

1 (with Fraker, Ross) A priori bounds for boundary sets, 29 (1971), 313-318.

Becker, James C.

1 (with Glover, H. H.) Note on the embedding of manifolds in Euclidean space, 27 (1971), 405-410. 
Bedford, Eric

1 Path maps and continuity, 24 (1970), 112-115.

$54 \mathrm{C05}$

Bednarek, Alexander R.

1 (with Whaley, Thomas P.) Binary relations on sets of regular cardinality, 23 (1969), 455-457.

Behzad, Mehdi

04A05 04A10 06A05 06A10 06A20; 02F10 04A20 20M35 68A25 94A30

1 A characterization of total graphs, 26 (1970), 383-389.

$05 C 99$

Beineke, Lowell W.

1 (with Schwartz, Benjamin L.) Locally finite self-interchange graphs, 27 (1971), 8-12.

Bellamy, David P.

$05 C 99$

1 (with Davis, Harvey S.) Continuum neighborhoods and filterbases, 27 (1971) 371-374.

$54 \mathrm{~F} 15$

2 Mappings of indecomposable continua, 30 (1971), 179-180.

Beller, E.

$54 \mathrm{C} 05$ 54F $20 ; 54 \mathrm{C} 15$

1 (with Newman, Donald J.) An $l_{1}$ extremal problem for polynomials, 29 (1971), 474-481. 30A06 42A04

2 Polynomial extremal problems in $L^{p}, 30$ (1971), 249-259.

30A06 42A04; 30A40

Belna, Charles L.

1 A necessary condition for principal cluster sets to be void, 24 (1970), 90-91.

$30 \mathrm{~A} 72$

2 The n-separated-arc property for homeomorphisms, 24 (1970), 98-99. 30A72 Benard, Mark

1 Quaternion constituents of group algebras, 30 (1971), 217-219.

16A26 20C15; 16A40 20C05

Bennett, Harold R.

1 (with Lutzer, David J.) Separability, the countable chain condition and the Lindelöf property in linearly orderable spaces, 23 (1969), 664-667.

54A25 54F05; 06A45 54D20

2 On Arhangel'skii's class MOBI, 26 (1970), 178-180. 54C10 54D20; 54E35

3 A note on point-countability in linearly ordered spaces, 28 (1971), 598-606.

54D15 54D20 54E35 54F05; 54D35 54F65

4 (with Berney, E. S.) Subparacompactness and $G_{\delta}$-diagonals in Arhangel'skiīs class MOBI, 30 (1971), 573-577. 54C10 54D20; 54E30

Bennett, Ralph

1 (with Transue, William R. R.) On embedding cones over circularly chainable continua, 21 (1969), 275-276.

Benson, Clark T.

1 (with Grove, Larry C.) Generators and relations for Coxeter groups, 24 (1970), 545-547.

20F05 20H 15

Benson, Donald C.

1 (with Kreith, Kurt) On abstract Pruefer transformations, 26 (1970), 137-140. 
Berberian, Sterling K.

1 Trace and the convex hull of the spectrum in a von Neumann algebra of finite class, 23 (1969), 211-212.

2 Some conditions on an operator implying normality. II, 26 (1970), 277-281.

Bergman, George M.

1 A weak Nullstellensatz for valuations, 28 (1971), 32-38.

Bernardi, S. D.

1 The radius of univalence of certain analytic functions, 24 (1970), 312-318.

$13 \mathrm{~A} 15 ; 12 \mathrm{~J} 20$

Berndt, Bruce C.

1 On the zeros of the Riemann zeta-function, 22 (1969), 183-188; Erratum: 24 (1970), 839.

2 The functional equation of some Dirichlet series, 29 (1971), 457-460. $\quad 10 \mathrm{H} 10$ Berney, E. S.

1 A regular Lindelöf semimetric space which has no countable network, 26 (1970), 361-364.

54D20 54E25 54G20; 54B10 54D15

2 (with Bennett, Harold R.) Subparacompactness and $G_{8}$-diagonals in Arhangel'skii's class MOBI, 30 (1971), 573-577. 54C10 54D20; 54E30

Bernfeld, Stephen R.

1 Liapunov functions and global existence without uniqueness, 25 (1970), 571-577. 34A 15 34D20

2 (with Lasota, Andrzej) Quickly oscillating solutions of autonomous ordinary differential equations, 30 (1971), 519-526.

34C15 34D05

Bernhardt, Robert L.

1 Splitting hereditary torsion theories over semiperfect rings, 22 (1969), 681-687.

Bernstein, Herbert $\mathbf{J}$. $18 \mathrm{E} 40 ; 16 \mathrm{~A} 36$

1 CCN-groups of order divisible by three primes, 22 (1969), 202-205. 20D99 Bernstein, Leon

1 An explicit summation formula and its application, 25 (1970), 323-334. 10F20 Bhattacharya, P. B.

1 (with Jain, S. K.) Rings having solvable adjoint groups, 25 (1970), 563-565.

\section{Białynicki-Birula, Andrzej}

16A22; 16A12 16A42

1 On the equivalence of integral representation of groups, 26 (1970), 371-377.

Biles, Charles M.

16A50 18H15 20E35; 16A64 16A74 20C10

1 Wallman-type compactifications, 25 (1970), 363-368. 54D35; 54F05 54G05 Billis, M. J.

1 A note on a theorem of B. H. Neumann and S. Yamamuro, 22 (1969), 439-440.

Bilyeu, Russell, G.

20E30; 02H15

1 Metric definition of the linear structure, 25 (1970), 205-206.

46B99

Bing, R. H.

1 Retractions onto ANR's, 21 (1969), 618-620.

54C55 57A15

Birman, Joan S. 
1 Automorphisms of the fundamental group of a closed, orientable 2-manifold, 21 (1969), 351-354.

20E40 55A05 57A05

Blair, David E.

1 (with Stone, Anthony P.) A note on the holonomy group of manifolds with certain structures, 21 (1969), 73-76.

53B20

Blau, Harvey I.

1 An inequality for complex linear groups of small degree, 28 (1971), 405-408.

Block, Henry David

$20 \mathrm{C} 1520 \mathrm{G} 20$

1 (with Levin, Simon A.) On the boundedness of an iterative procedure for solving a system of linear inequalities, 26 (1970), 229-235.

Blum, Peter

15A39 65F10; 65K05 93C40 94A30

1 Extending differential specializations, 24 (1970), 471-474.

$12 \mathrm{H05}$

Blumenthal, Robert George

1 The spectrum of a function algebra, 25 (1970), 343-346.

$46 \mathrm{~J} 1046 \mathrm{~J} 20$

Boardman, John

1 Stable homotopy theory is not self-dual, 26 (1970), 369-370.

55B20 55D25 55D99; 55E05

Bobisud, Larry E.

1 Oscillation of nonlinear second-order equations, 23 (1969), 501-505. 34C15

Boehme, T. $\mathbf{K}$.

1 (with Weiss, Max L.) One-sided boundary behavior for certain harmonic functions, 27 (1971), 280-288.

$31 \mathrm{~A} 20$

2 (with Weiss, Max L.) Extensions of Fatou's theorem to tangential asymptotic values, 27 (1971), 289-298.

30 A98 31A20

Bogar, Gary A.

1 Properties of two point boundary value functions, 23 (1969), 335-339. 34B05 Bogart, Kenneth P.

1 Idempotent Noether lattices, 22 (1969), 127-128.

06A50; 06A40

2 Nonimbeddable Noether lattices, 22 (1969), 129-133. O6A30 06A50; 13C05

3 Small regular local Noether lattices. I, 25 (1970), 423-428. O6A50; 13H05 Bogdanowicz, Witold M.

1 Analytic continuation of holomorphic functions with values in a locally convex space, 22 (1969), 660-666.

30A14 30A96

Bookhout, Glenn A.

1 Metric dimension of complete metric spaces, 24 (1970), 754-759.

Borges, Carlos J. R.

54F45 54G20; 54E50

1 On continuously semimetrizable and stratifiable spaces, 24 (1970), 193-196.

54G20 54G99

2 Metrizability of adjunction spaces, 24 (1970), 446-451. 54B17 54E35; 54E60

Borrego, Joseph T.

1 Point-transitive actions by a standard metric thread, 23 (1969), 261-265.

Borwein, David

$22 \mathrm{~A} 15$

1 On absolute Borel-type methods of summability, 24 (1970), 85-89.

40F05 40G10 
Bosch, W.

1 (with Krajkiewicz, P.) Polyanalytic functions with equal modulus, 23 (1969), 127132. $30 \mathrm{~A} 94$

2 (with Krajkiewicz, P.) The big Picard theorem for polyanalytic functions, 26 (1970), 145-150.

Bose, Anil Kumar

30A96; 30A02 30A70

1 A note on entire functions of bounded index, 21 (1969), 257-262.

\section{Bouldin, Richard}

$30 \mathrm{~A} 64 ; 30 \mathrm{~A} 62$ 30A66

1 The Weyl essential spectrum, 28 (1971), 531-536.

47A10 47B30

Bownds, John M.

1 (with Metcalf, Frederic T.) An extension of the Nagumo uniqueness theorem, 27 (1971), 313-316.

Boyd, David W.

1 Transcendental numbers with badly distributed powers, 23 (1969), 424-427.

Brady, Michael M.

$10 \mathrm{~F} 40$

1 Meromorphic solutions of a system of functional equations involving the modular group, 30 (1971), 271-277.

Bragg, Louis $\mathbf{R}$.

1 (with Dettman, John W.) A class of related Dirichlet and initial value problems, 21 (1969), 50-56.

35A22 35G15

2 (with Dettman, John W.) Multinomial representation of solutions of a class of Brand, Louis singular initial value problems, 21 (1969), 629-634. $35 \mathrm{~A} 20$ 35C10 35Q05

1 On the product of singular symmetric matrices, 22 (1969), 377. 15A18 15A57 Brandstein, A. G.

1 A class of hypo-Dirichlet algebras, 28 (1971), 501-504. $46 \mathrm{~J} 1046 \mathrm{~J} 20$ Brasher, Russell G.

1 A separation theorem for manifolds, 23 (1969), 242-245.

55B05 55C99

2 The homology sequence of the double covering; Betti numbers and duality, 23 (1969), 714-717.

Bredon, Glen E.

55A 10 55B25 55C99

1 The set of nonprincipal orbits of an action on $E^{n}, 23$ (1969), 254-255.

57E 15 57E25

2 Open, finite to one maps of contractible polyhedra onto a sphere, 24 (1970), 251-257.

$54 \mathrm{C} 10$ 55C $99 ; 55 \mathrm{C} 3555 \mathrm{~F} 25$

3 Another exotic action on $E^{n}, 25$ (1970), 437-438.

$55 \mathrm{C} 35$ 57E 15 57E25

4 Counterexamples on the rank of a manifold, 27 (1971), 592-594.

Brender, Allan

55C99 57D25 57E15 58A30

1 Inertial and bordism properties of spheres, 27 (1971), 209-212. $\quad$ 57D60 57D90

Brenner, Jöel Lee

1 (with Brown, William G.) On a hierarchy of generalized diagonal dominance properties for complex matrices, 25 (1970), 906-911.

$15 \mathrm{~A} 15$

2 Bounds for classical polynomials derivable by matrix methods, 30 (1971), 353-362. 


\section{Breuer, Shlomo}

1 (with Gottlieb, David) Explicit characterization of spherical curves, 27 (1971), 126-127.

34A05 53A05

2 A characterization of order topologies by means of minimal $T_{O^{-t o p o l o g i e s,}} 27$ (1971), 161-167.

06A45 54F05 54F65; 54D05 54D10

3 (with Gottlieb, David) Separation of roots and oscillation in ordinary linear differential equations of second order, 29 (1971), 487-493.

Brewer, J. W.

1 (with Arnold, Jimmy T.) Kronecker function rings and flat $D[X]$-modules, 27 (1971), 483-485.

13B25 13F05

\section{Brillhart, John}

1 (with Carlitz, Leonard) Note on the Shapiro polynomials, 25 (1970), 114-118.

Brooks, James K.

33A70; 42A 16

1 Decomposition theorems for vector measures, 21 (1969), 27-29.

$28 \mathrm{~A} 45$

Brown, Arlen

1 A remark on set theory, 25 (1970), 920-921.

02K20 04A25

Brown, 3. M.

1 A general three-series theorem, 28 (1971), 573-577.

Brown, Edward M.

60F15 60G45; 60E05 60G50

1 A note on punctured disks in a 2-manifold, 22 (1969), 471-472.

Brown, Ezra

$1 A$ theorem on biquadratic reciprocity, 30 (1971), 220-222.

55A05 57A05

Brown, Herbert I.

1 (with Kerr, Donald R., Jr. and Stratton, Howard H., Jr.) The structure of $B[c]$ and extensions of the concept of conull matrix, 22 (1969), 7-14. 46L20; 40H05

2 (with Stratton, Howard H., Jr.) Conullity of operators on some FK-spaces, 25 (1970), 717-727.

40H05 40J05 46A05 46A45

Brown, J. W.

1 New generating functions for classical polynomials, 21 (1969), 263-268.

2 (with Goldberg, J. L.) Index-dependent parameters of Laguerre and related polynomial sets, 25 (1970), 852-855.

$33 \mathrm{~A} 65 ; 33 \mathrm{~A} 30$

Brown, Jack B.

1 Negligible sets for real connectivity functions, 24 (1970), 263-269. 26A15

Brown, James Ward

1 On the Sheffer A-type of certain modified polynomial sets, 23 (1969), 718-722.

$33 \mathrm{~A} 30$

Brown, Leon

1 Subalgebras of $L_{\infty}$ of the circle group, 25 (1970), 585-587. $43 \mathrm{~A} 1546 \mathrm{~J} 30$

Brown, Robert F.

1 A fixed point theorem for open Q-acyclic n-manifolds, 21 (1969), 621-622.

55C20 57A15; 54F40 54H 25

2 H-manifolds have no nontrival idempotents, 24 (1970), 37-40. 55D45 57C99

3 Divisible H-spaces, 26 (1970), 185-189. 22A15 54H25 55C20; 55D45 57A65 


\section{Brown, Terrence $\mathbf{J}$.}

1 A recursion formula for finite partition lattices, 22 (1969), 124-126.

05A05; 04A20 10A20

Brown, William C.

1 (with Ingraham, Edward C.) A characterization of semilocal inertial coefficient rings, 26 (1970), 10-14. 13H99 13J15 16A16

Brown, William G.

1 (with Brenner, Joel Lee) On a hierarchy of generalized diagonal dominance properties for complex matrices, 25 (1970), 906-911.

15A 15

Brualdi, Richard A.

1 Induced matroids, 29 (1971), 213-221.

05B35 05C20; 05A05

Brungs, Hans-Heinrich

1 Overrings of principal ideal domains, 28 (1971), 44-46. 16A04; 16A08

Bruning, L. M.

1 (with Leavitt, William G.) Minimal generating sets for free modules, 27 (1971), 441-445.

$16 \mathrm{~A} 64 ; 16 \mathrm{~A} 48$

Brunk, Hugh D.

1 Correction to "On an extension of the concept conditional expectation", 23 (1969), 727.

60A05

Bryan, Robert Neff

1 A nonhomogeneous linear differential system with interface conditions, 22 (1969), 270-276.

$34 \mathrm{~B} 10$

Bryant, John L.

1 On embeddings of compacta in euclidean space, 23 (1969), 46-51.

Brydak, Dobieslaw

57A 15 57A35; 57A 30

1 On the stability of the functional equation $\phi[f(x)]=g(x) \phi(x)+F(x), 26(1970)$, 455-460.

39A25

Buchman, E. $O$.

1 Property $P_{3}$ and the union of two convex sets, 25 (1970), 642-645.

Bunce, John

$52 \mathrm{~A} 2052 \mathrm{~A} 30$

1 Characters on singly generated $C^{*}$-algebras, 25 (1970), 297-303.

46L05 47A10; 47B20

2 A note on two-sided ideals in $C^{*}$-algebras, 28 (1971), 635.

46L05

3 The joint spectrum of commuting nonnormal operators, 29 (1971), 499-505.

Burgess, C. E.

47A10 47B20

1 (with Cannon, J. W.) Tame subsets of spheres in $E^{3}, 22$ (1969), 395-401.

Burke, Dennis $\mathrm{K}$.

1 On subparacompact spaces, 23 (1969), 655-663. 54D20; 54G20

Burns, R. G.

1 A note on free groups, 23 (1969), 14-17.

20E05

Burris, $\mathbf{S}$.

1 (with Nelson, Evelyn) Embedding the dual of $\Pi_{m}$ in the lattice of equational classes of commutative semigroups, 30 (1971), 37-39.

Burton, T. A.

08A15 20M05 
1 (with Grimmer, R. C.) On continuability of solutions of second order differential equations, 29 (1971), 277-283. 34A15 34C15

Busby, Robert C.

1 On a theorem of Fell, 30 (1971), 133-140. 22D30 46L25

Buschman, R. G.

1 Identities involving products of number-theoretic functions, 25 (1970), 307-309.

$10 \mathrm{~A} 20$

Busenberg, Stavros

1 Iterative solution of a Wiener-Hopf problem in several variables, 29 (1971), 39-46. 32A10 45E10; 22A07 32A25 42A68

Bustoz, Joaquin

1 (with Atalla, Robert E.) On sequential cores and a theorem of $R$. R. Phelps, 21 (1969), 36-42. 40C05 40H05 46A45 54A25 54C10 54C15 54E50; 46E10

2 On regular matrices that induce the Gibbs phenomenon, 25 (1970), 481-487.

Butler, G. J.

1 An extremal property of simplices, 30 (1971), 556-560.

Butt, Shu-shih

1 On maximal nondetermining subalgebras of group algebras, 21 (1969), 427-436.

Byers, William P.

43A20 43A25

1 On a theorem of Preissmann, 24 (1970), 50-51. 53C20 55A05

Byrnes, J. S.

1 (with Newman, Donald J.) Completeness preserving multipliers, 21 (1969), 445-450.

$42 \mathrm{~A} 60$ 46E30

2 (with Newman, Donald J.) A lower Jackson bound on $(-\infty, \infty), 26$ (1970), 71-72.

Cain, George L., Jr.

1 Compactification of mappings, 23 (1969), 298-303. 54D35; 54C05 54C10

Cambanis, Stamatis

1 Bases in $L_{2}$ spaces with applications to stochastic processes with orthogonal increments, 29 (1971), 284-290. 42A56 46E20 60G10 60G15 60G17; 42A64

Canfell, M. J.

1 Uniqueness of generators of principal ideals in rings of continuous functions, 26 (1970), 574-578.

$13 \mathrm{~A} 05$ 54F45

Cannon, J. W.

1 (with Burgess, C. E.) Tame subsets of spheres in $E^{3}, 22$ (1969), 395-401.

57A10; 55A30

2 (with Wayment, S. G.) An imbedding problem, 25 (1970), 566-570.

54A20 54C25 54F15 57A10 57A15; 54C35 57A05

Cantrell, James C.

1 (with Price, Thomas and Rushing, T. B.) A class of embeddings of $S^{n-1}$ and $B^{n}$ in $R^{n}, 29$ (1971), 208-210.

57A15 57A45

Caradus, S. R.

1 Universal operators and invariant subspaces, 23 (1969), 526-527.

\section{Cardoso, Fernando}

47A 15 47A45

1 The identity of weak and strong extensions of pseudo-differential operators, 29 
(1971), 118-122.

$35 \mathrm{D} 1035 \mathrm{~S} 05$

Carlitz, Leonard .

1 (with Brillhart, John) Note on the Shapiro polynomials, 25 (1970), 114-118.

Carlson, B. C.

1 Inequalities for a symmetric elliptic integral, 25 (1970), 698-703.

Carns, Gail L.

33A25 41A30 65N99

1 A functor to ringed spaces, 29 (1971), 222-228.

06A70 12J15

Carpenter, R. L.

1 Uniqueness of topology for commutative semisimple F-algebras, 29 (1971), 113-117.

$46 \mathrm{H} 05$

2 Continuity of systems of derivations on F-algebras, 30 (1971), 141-146.

46H99; 30A98

Carruth, J. H.

1 (with Clark, C. E.) Compact totally ordered semigroups, 27 (1971), 199-204.

Catlin, Donald E.

1 Cyclic atoms in orthomodular lattices, 30 (1971), 412-418.

06A25 06A35; 06A23 06A40 46L99 47D99

Caughran, James G.

1 Zeros of analytic functions with infinitely differentiable boundary values, 24 (1970), 700-704.

30A72; 30A08 30A76

Causey, W. M.

1 The univalence of an integral, 27 (1971), 500-502. 30A34

Caveny, D. J.

1 (with Novinger, W. P.) Boundary zeros of functions with derivative in $H^{p}, 25$ (1970), 776-780.

30A78 30A80

Chacon, Rafael V.

1 Weakly mixing transformations which are not strongly mixing, 22 (1969), 559-562.

$28 \mathrm{~A} 65$

2 (with Akcoglu, M. A. and Schwartzbauer, T.) Commuting transformations and mixing, 24 (1970), 637-642.

$28 \mathrm{~A} 65$

Chacron, $\mathbf{M}$.

1 Direct product of division rings and a paper of Abian, 29 (1971), 259-262.

06A70 16A40; 16A48

Chambers, Graham A.

1 On the conjugacy of injectors, 28 (1971), 358-360. 20D10 Chambless, Donald A.

1 Representations of l-groups by almost-finite quotient maps, 28 (1971), 59-62.

06A60 06A65

Chandy, A. John

1 Rings generated by the inner-automorphisms of nonabelian groups, 30 (1971), 59-60.

20E15 20F55; 16A76

Chaney, Robin W.

1 On uniformly approximable Sidon sets, 21 (1969), 245-249.

43A25

Chang, John S. M.

1 Rotational approximation, 29 (1971), 623-624.

30A18 30A82 42A64

Chang, K. W. 
1 Remarks on a certain hypothesis in singular perturbations, 23 (1969), 41-45.

Chao, Chong-yun

1 Infinitely many nonisomorphic nilpotent algebras, 24 (1970), 126-133.

\section{Chatfield, J. A.}

16A22 17B30

1 (with Davis, Wilbor P.) Concerning product integrals and exponentials, 25 (1970), 743-747.

26A39 26A42 26A45; 45D05

Chazin, R. L.

1 Stable thickenings in the topological category, 29 (1971), 175-178.

55F35 57A15 57C10 57A99; 57C40

Chen, Bang-yen

1 On an inequality of T. J. Willmore, 26 (1970), 473-479; Erratum: 29 (1971), 627.

52A40 53A05; 53C45 53C65

2 Submanifolds in a euclidean hypersphere, 27 (1971), 627-628. 53A05 53C40

3 On an integral formula of Gauss-Bonnet-Grotemeyer, 28 (1971), 208-212.

$53 \mathrm{C} 4053 \mathrm{C} 65 ; 53 \mathrm{C} 20$

4 Minimal hypersurfaces in an m-sphere, 29 (1971), 375-380.

\section{Chen, Kuo-tsai}

53A10 53C40; 53A05 53B25

1 A sufficient condition for nonabelianness of fundamental groups of differentiable manifolds, 26 (1970), 196-198.

55A05 55B45; 55A 10

Chen, Su-shing

1 Theorems of Accola type for higher dimensional manifolds, 30 (1971), 479-483.

Cherkas, Barry M.

32C10 32M05

1 Compactness in $L_{\infty}$ spaces, 25 (1970), 347-350. 46E30

Chernoff, Paul R.

1 Elements of a normed algebra whose $2^{n}$ th powers lie close to the identity, 23 (1969), 386-387.

Chew, Kim-peu

1 A characterization of $N$-compact spaces, 26 (1970), 679-682.

54C25 54D60; 06A40

Chewning, W. C.

1 Can a 2-coherent Peano continuum spearate $E^{3}, 30$ (1971), 185-188.

Choe, Tae Ho

1 The breadth and dimension of a topological lattice, 23 (1969), 82-84.

\section{Chou, Ching}

06A35 54F05 54F45; 55C10 57A 15

1 On the size of the set of left invariant means on a semigroup, 23 (1969), 199-205.

2 On a conjecture of $E$. Granirer concerning the range of an invariant mean, 26 (1970), 105-107.

43A07; 28A70

3 On a geometric property of the set of invariant means on a group, 30 (1971), 296-302. 28A70 43A07 46A05; 46N05

Chow, Kwang-nan

1 (with Glasner, Moses) Bounded in the mean solutions of $\Delta u=P u$ on Riemannian manifolds, 26 (1970), 261-265. 
Chow, T. R.

1 The spectral radius of a direct integral of operators, 26 (1970), 593-597.

47A10 47A60

2 (with Gilfeather, Frank) Functions of direct integrals of operators, 29 (1971), 325-330.

47A20 47C15

Chrislock, J. L.

1 A certain class of identities on semigroups, 21 (1969), 189-190. 20M99

Chui, Charles Kam-tai

1 Measures of $N$-fold symmetry for convex sets, 26 (1970), 480-486. $\quad 52 A 10$

Chvátal, Václav

1 Hypergraphs and Ramseyian theorems, 27 (1971), 434-440.

05A05 05C15 05C30

Chwe, Byoung-song

1 (with Neggers, Joseph) On the extension of linearly independent subsets of free modules to bases, 24 (1970), 466-470. 16A64; 16A08

Cima, Joseph A.

1 On the dual of Hornich's space, 22 (1969), 102-103. 30A98 46E15

Clancey, Kevin F.

1 Examples of nonnormal seminormal operators whose spectra are not spectral sets, 24 (1970), 797-800.

$47 \mathrm{~A} 25$ 47B20

2 Seminormal operators with compact self-commutators, 26 (1970), 447-454.

Clark, C. E.

47B 10

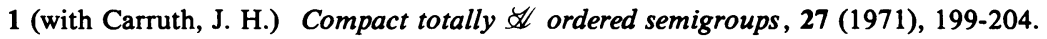

20M10 22A15

Clements, G. F.

1 Sets of lattice points which contain a maximal number of edges, 27 (1971), 13-15.

Closs, Mike P.

$05 \mathrm{~A} 05$ 05A 15

1 Homogeneous almost tangent structures, 23 (1969), 237-241. 53C15 53C30

Clough, Robert R.

1 Calculation of $H^{*}\left(B B O[k] ; Z_{2}\right), 24(1970), 32-36 . \quad 55 F 4055 G 1055 G 45$ Cobb, John

1 On ordering infinitely many small homeomorphisms, 23 (1969), 64-67.

Cochran, Allan C.

54B99 57A15; 06A05 54F45

1 Weak A-convex algebras, 26 (1970), 73-77.

46H20; 46A05

2 Topological algebras and Mackey topologies, 30 (1971), 115-119.

Cohen, Marshall $\mathbf{M}$.

46H05 46H20; 46A09

1 Simplicial and piecewise linear collapsibility, 24 (1970), 649-650.

Cohn, John H. E.

1 Sums of cubes of Gaussian integers, 29 (1971), 426.

57C05 57C99 Coleman, Donald B.

1 (with Passman, D. S.) Units in modular group rings, 25 (1970), 510-512.

$20 \mathrm{C05}$

2 (with Enochs, Edgar E.) Isomorphic polynomial rings, 27 (1971), 247-252. 


\section{Collins, Heron Sherwood}

1 (with Summers, W. H.) Some applications of Hewitt's factorization theorem, 21 (1969), 727-733.

$46 \mathrm{H} 05$ 46H25; 47B05

\section{Collins, P. J.}

1 Concordant mappings and the concordant-dissonant factorization of an arbitrary continuous function, 27 (1971), 587-591.

54B15 54C10 54D05; 54E45

Comer, Stephen D.

1 (with Le Tourneau, J. J.) Isomorphism types of infinite algebras, 21 (1969), 635-639.

Comfort, W. W.

$08 \mathrm{~A} 15$

1 Closed Baire sets are (sometimes) zero-sets, 25 (1970), 870-875.

28A05 54A99 54H05; 54D20 54D35

Conlon, Lawrence

1 Remarks on commuting involutions, 22 (1969), 255-257.

20F55 22E10; $53 \mathrm{C} 35$

Connelly, Robert

1 A new proof of Brown's collaring theorem, 27 (1971), 180-182.

57A35; 57C35

\section{Connors, Edward A.}

1 The structure of $O^{\prime} / \Omega$ over local fields of characteristic 2,22 (1969), 596-599.

$10 \mathrm{C05}$

\section{Conrad, Bruce}

1 Extending free circle actions on spheres to $S^{3}$ actions, 27 (1971), 168-174. 57C25 57D10 57D20 57D60 57E25 57E99; 57C20 57E10 57E15

Constantin, Gheorghe

1 (with Istrăţescu, Ioana) Some remarks on structure of Riesz operators, 21 (1969), 455-458.

47A65 47B05; 47B20

Conway, John B.

1 On algebras of operators with totally ordered lattice of invariant subspaces, 28 (1971), 163-168.

46C15 47A 15

Cook, Thurlow A.

1 Weakly equicontinuous Schauder bases, 23 (1969), 536-537.

$46 \mathrm{A35}$

Cooke, Roger

1 A Cantor-Lebesgue theorem in two dimensions, 30 (1971), 547-550.

Cooper, G.

1 (with Amoroso, S.) The Garden-of-Eden theorem for finite configurations, 26 (1970), 158-164.

05B30 68A25

Cooper, J. B.

1 The strict topology and spaces with mixed topologies, 30 (1971), 583-592.

Cornelius, E. F., Jr.

46A05; 46A09 46E 10

1 Note on quasi-decompositions of irreducible groups, 26 (1970), 33-36. 20K20

Cossey, John

1 (with Whittemore, Alice) On the Frattini subgroup, 21 (1969), 699-702; Addendum: 27. (1971), 63-64.

20F15 20F30

Costich, O. L.

1 (with Doyle, Patrick H. and Galewski, D. E.) A characterization of punctured open 
3-cells, 28 (1971), 295-298.

57A10; 55A40

Coury, John E.

1 On the measure of zero sets of coordinate functions, 25 (1970), 16-20.

22C05 22D10; 20C15 28A70

Coven, Ethan M.

1 Compactness of limit sets and semiorbit closures, 23 (1969), 120-122. 54H20

2 (with Reddy, William L.) Limit set equivalences of replete semigroups, 23 (1969), 625-630.

54H15 54H20; 22B99

Cox, Raymond $\mathrm{H}$.

1 (with Powell, Robert E.) Regularity of net summability transforms on certain linear topological spaces, 21 (1969), 471-476.

$40 \mathrm{~J} 05$

Cox, S. H., Jr.

1 Determinantal rank and flat modules, 22 (1969), 104-108. 13C10 18G05

Crabtree, Douglas E.

1 (with Haynsworth, Emilie V.) An identity for the Schur complement of a matrix, 22 (1969), 364-366.

15A24

Crandall, Michael G.

1 Norm preserving extensions of linear transformations on Hilbert spaces, 21 (1969), 335-340.

47A20; 47B44

Crawford, Albert L.

1 (with Jobe, John) A characterization of hereditarily indecomposable continua, 27 (1971), 205-208.

54E30 54F20; 54D05 54E35

Creede, Geoffrey D.

1 Embedding of complete Moore spaces, 28 (1971), 609-612.

54C25 54E30; 54D30 54D35

Crittenden, Richard B.

1 (with Vanden Eynden, C. L.) Any $n$ arithmetic progressions covering the first $2^{n}$ integers cover all integers, 24 (1970), 475-481.

10A 10

Cronin-Scanlon, Jane

1 Quasilinear systems with several periodic solutions, 30 (1971), 107-111.

$34 \mathrm{C} 15$ 34C25

Cullen, Michael R.

1 Meromorphic functions which cluster on the boundary, 23 (1969), 588-589.

30A72; 30A68 30A76

Cunsolo, J.

1 (with Akcoglu, M. A.) An ergodic theorem for semigroups, 24 (1970), 161-170.

Cuppens, Roger

28A65

1 On the decomposition of infinitely divisible characteristic functions with continuous Poisson spectrum, 21 (1969), 145-152.

60B15 60E05 62E10

Curtis, Douglas W.

1 Deficient subsets in locally convex spaces, 21 (1969), 289-295.

46A05 57A17; 55D99

2 (with McCoy, R. A.) Stable homeomorphisms on infinite-dimensional normed linear spaces, 28 (1971), 496-500.

58B05

Curtis, Morton L.

1 (with Dugundji, John) Groups which are cogroups, 22 (1969), 235-237.

Cusick, T. W.

22C05; 54F60 
1 Sums and products of continued fractions, 27 (1971), 35-38.

$10 \mathrm{~A} 30$

2 (with Lee, R. A.) Sums of sets of continued fractions, 30 (1971), 241-246.

Cutler, Doyle 0.

$10 \mathrm{~F} 20$

1 (with Winthrop, Joel) A note on a paper of Paul Hill and Charles Megibben, 22 (1969), 428-429.

$20 \mathrm{~K} 10$

2 Another summable $C_{\Omega}$-group, 26 (1970), 43-44. 20K10

Cutler, William H.

1 Negligible subsets of infinite dimensional Fréchet manifolds, 23 (1969), 668-675. 57A20 58B05 58B20; 54E35

Darst, Richard B.

1 Properties of vector valued finitely additive set functions, 23 (1969), 528-535

$28 \mathrm{~A} 45$

2 (with Pollard, Harry) An inequality for the Riemann-Stieltjes integral, 25 (1970), 912-913.

26A42 26A84 26A86

3 On the 1-1 sum of two Borel sets, 25 (1970), 914.

04A15 28A05 54H05; 02K30 54H05

$4 C^{\infty}$-functions need not be bimeasurable, 27 (1971), 128-132.

02K30 04A15 26A93 28A05 54H05; 28A20

5 A characterization of bimeasurable functions in terms of universally measurable sets, 27 (1971), 566-571.

02K25 02K30 04A15 04A30 26A21 28A05 28A10 28A25 28A60 54H05,

26A93 28A35

Das, K. M.

1 An inequality similar to Opial's inequality, 22 (1969), 258-261.

26A84

Das, Minaketan

1 Real zeros of a random sum of orthogonal polynomials, 27 (1971), 147-153.

26A78 33A65 42A52 60F99 62E20; 60E05

Daverman, Robert J.

1 A new proof for the Hosay-Lininger theorem about crumpled cubes, 23 (1969), 52-54.

Davie, Alexander $\mathbf{M}$

$57 \mathrm{~A} 10$

1 (with $\emptyset \mathrm{ksendal}$, B. K.) Rational approximation on the union of sets, 29 (1971), 581-584.

$30 \mathrm{~A} 82$ 46J15

Davies, Roy 0.

1 (with Hayes, Allan and Rousseau, George) Complete lattices and the generalized Cantor theorem, 27 (1971), 253-258.

06A10; 06A20

Davis, Harvey S.

1 (with Bellamy, David P.) Continuum neighborhoods and filterbases, 27 (1971), 371-374.

54F15

Davis, R. D.

1 (with Wishart, E.) Galois extensions and the ramification sequence of some wildly ramified $\pi$-adic fields, 30 (1971), 212-216. 12B10 12B15

Davis, Robert

1 Component functors, 24 (1970), 396-400.

$18 \mathrm{C} 15 ; 18 \mathrm{~B} 05$

2 Free coalgebras in a category of rings, 25 (1970), 155-158; Erratum: 25 (1970), 922.

13D99 18C10; 08A10 
3 Semiadjoint functors and quintuples, 27 (1971), 477-482.

18A40; $14 \mathrm{~K} 99$

Davis, Wilbor P.

1 (with Chatfield, J. A.) Concerning product integrals and exponentials, 25 (1970), 743-747. 26A39 26A42 26A45; 45D05

Davis, William J.

1 Basis preserving maps, 22 (1969), 34-36.

46B 15

Davitt, Richard M.

1 The automorphism group of a finite metacyclic p-group, 25 (1970), 876-879.

20D15 20D45

2 (with Otto, Albert D.) On the automorphism group of a finite p-group with the central quotient metacyclic, 30 (1971), 467-472. 20D45

Daykin, David E.

1 Generalisation of the Muirhead-Rado inequality, 30 (1971), 84-86. 26A86

Dean, Richard A.

1 (with Evans, Trevor) A remark on varieties of lattices and semigroups, 21 (1969), 394-396.

06A20 08A15 20M05

Deddens, James A.

1 Commuting unilateral shifts, 25 (1970), 96-99.

$47 \mathrm{~A} 45$

2 Every isometry is reflexive, 28 (1971), 509-512.

$47 \mathrm{~A} 15 ; 47 \mathrm{~A} 50$

De Marco, Giuseppe

1 (with Wilson, R. G.) Realcompactness and purtitions of unity, 30 (1971), 189-194. 54C40 54D60; 54A25 54D20 54E15

2 (with Orsatti, Adalberto) Commutative rings in which every prime ideal is contained in a unique maximal ideal, 30 (1971), 459-466. 13A10 13A15; 54C40

DeMarr, Ralph E.

1 Convergence of a sequence of powers, 23 (1969), 401-403.

DeMeyer, Frank R.

06A10 06A70 15A51

1 Groups with an irreducible character of large degree are solvable, 25 (1970), 615-617.

Deo, S. G.

20C15 20D10

1 (with Murdeshwar, M. G.) On a system of integral inequaliţies, 26 (1970), 141-144.

$34 \mathrm{~A} 40$

2 On vector Lyapunov functions, 29 (1971), 575-580. 34D20; 34A40

Dettman, John W.

1 (with Bragg, Louis R.) A class of related Dirichlet and initial value problems, 21 (1969), 50-56.

35A22 35G15

2 (with Bragg, Louis R.) Multinomial representation of solutions of a class of singular initial value problems, 21 (1969), 629-634.

35A20 35C10 35Q05

Deutsch, Emeric

1 On vectorial norms and pseudonorms, 28 (1971), 18-24.

$15 \mathrm{~A} 60 ; 46 \mathrm{~B} 10$

Diaz, Joaquin B.

1 (with Young, Eutiquio C.) Uniqueness of solutions of certain boundary value problems for ultrahyperbolic equations, 29 (1971), 569-574.

Dickey, R. W.

$35 \mathrm{G} 15 ; 35 \mathrm{~A} 05$ 35L20

1 Infinite systems of nonlinear oscillation equations related to the string, 23 (1969), 459-468. 
Dickman, R. F., Jr.

1 A theorem on one-to-one mappings onto the plane, 21 (1969), 119-120.

57A05; 54F25 54F65

Dickson, Spencer E.

1 (with Fuller, Kent R.) Commutative $Q F-1$ Artinian rings are $Q F, 24$ (1970), 667 670.

13E10 16A36

Dietrich, William E., Jr.

$1 A$ note on the ideal structure of $C(X), 23$ (1969), 174-178. 46J10 46J20

Dikshit, H. P.

1 Summability of a sequence of Fourier coefficients by a triangular matrix transformation, 21 (1969), 10-20.

42A24; $42 \mathrm{~A} 40$

2 Absolute summability of a series associated with a Fourier series, 22 (1969), 316-318.

42A28

3 A Tauberian theorem for the $(C, 1)(N, 1 /(n+1))$ summability method, 25 (1970), 391-392.

40E05; 40G05

Dinculeanu, Nicolae

1 (with Lewis, Paul W.) Regularity of Baire measures, 26 (1970), 92-94.

28A45; 26A21 28A10

Ditzian, Zeev

1 On Hille's first_exponential formula, 22 (1969), 351-355. 47D05

2 Note on Hille's exponential formula, 25 (1970), 351-352. 47D05

Dixon, John D.

1 A brief proof of Cauchy's integral theorem, 29 (1971), 625-626.

Dokovic, Dragomir ̌̌.

1 Note on nonnegative matrices, 25 (1970), 80-82.

$30 \mathrm{~A} 86$

2 A determinantal inequality for projectors in a unitary space, 27 (1971), 19-23.

Dollinger, Michael B.

1 Nuclear topologies consistent with a duality, 23 (1969), 565-568.

Dornhoff, Larry

1 Jordan's theorem for solvable groups, 24 (1970), 533-537. 20D10 20H20

Doss, Raouf

1 Elementary proof of a theorem of Helson, 27 (1971), 418-420. 43A05 43A30

Dotson, W. G., Jr.

1 On the solution of linear functional equations by averaging iteration, 25 (1970), 504-506.

47A50

Douglas, Ronald George

1 (with Sarason, Donald) Fredholm Toeplitz operators, 26 (1970), 117-120.

47B30 47B35

\section{Downing, J. Scott}

1 Decomposing compact 3-manifolds into homeomorphic handlebodies, 24 (1970), 241-244.

57A10 57C99 57D65

Doyle, Patrick H.

1 (with Costich, O. L. and Galewski, D. E.) A characterization of punctured open 3-cells, 28 (1971), 295-298.

57A10; $55 \mathrm{~A} 40$

Drobot, Vladimir 
1 Overconvergence and $(C, 1)$ summability, 25 (1970), 13-15. 30A10 30A12 Dubuc, Serge

1 Positive harmonic functions of a branching process, 21 (1969), 324-326.

$31 \mathrm{C} 2060 \mathrm{~J} 05$ 60J80; 60J45

Dudley, R. M.

1 Convergence of sequences of distributions, 27 (1971), 531-534. 46F05

Dugundji, John

1 (with Curtis, Morton L.) Groups which are cogroups, 22 (1969), 235-237.

$22 \mathrm{C} 05 ; 54 \mathrm{~F} 60$

Duncan, R. L.

1 On the factorization of integers, 25 (1970), 191-192. 10A25

2 Some applications of the Turán-Kubilius inequality, 30 (1971), 69-72. $\quad$ 10K20 Dunkl, Charles F.

1 Functions that operate in the Fourier algebra of a compact group, 21 (1969), 540-544. 43A15 43A20 43A75

2 (with Ramirez, Donald E.) Multipliers on compact groups, 28 (1971), 456-460.

Duren, Peter $\mathbf{L}$.

1 On the multipliers of $H^{p}$ spaces, 22 (1969), 24-27. 30A78

2 Coefficients of meromorphic schlicht functions, 28 (1971), 169-172. $\quad 30$ A34 Dyer, James A.

1 (with Johnson, W. B.) Isomorphisms generated by fundamental and total sets, 22 (1969), 330-334.

$46 \mathrm{~A} 35$

Dykstra, Richard L.

1 A note on a theorem by H. D. Brunk, 24 (1970), 171-174. 47B55

Eastham, M. S. P.

1 On the gaps in the spectrum associated with Hill's equation, 21 (1969), 643-647.

$34 \mathrm{~B} 25$

Eaton, William T.

1 Taming a surface by piercing with disks, 22 (1969), 724-727.

Eaves, B. Curtis

57A10; 54F35 55A35 57A05

1 An odd theorem, 26 (1970), 509-513.

$54 \mathrm{~A} 25$ 55C20; 54H25

Ebin, David G.

1 Completeness of Hamiltonian vector fields, 26 (1970), 632-634.

Edelson, Allan L.

$34 \mathrm{~A} 10$ 34A35 34C35

1 Real line bundles on spheres, 27 (1971), 579-583. 55E20 55E99 55F25 55F50 Edmondson, Don E.

1 Modularity in topological lattices, 21 (1969), 81-82. $\quad$ 06A30 54F05; 22A15 Edwards, J. R.

1 A sufficient condition that the limit of a sequence of continuous functions be an embedding, 26 (1970), 224-225.

54A20 54C25 54E35 54E50; 54C05

2 (with Wayment, S. G.) A v-integral representation for linear operators on a space of continuous vector valued functions, 30 (1971), 260-262. 28A45 46E10

3 (with Wayment, S. G.) A v-integral representation for the continuous linear operators on spaces of continuously differentiable vector-valued functions, 30 (1971), 263 270.

28A45 46E10 


\section{Efroymson, Gustave}

1 The cohomology ring of a finite group scheme, 26 (1970), 567-570. 14L20 Eifler, Larry

1 The slice product of function algebras, 23 (1969), 559-564. 46J10; 30A98 Einhorn, Sheldon J.

1 Functions positive definite in $C[0,1], 22$ (1969), 702-703. 26A96; 42A88

Eisenberg, Murray

1 Embedding a transformation group in an automorphism group, 23 (1969), 276-281.

$54 \mathrm{H} 15 ; 54 \mathrm{H} 20$

Eklof, Paul C.

1 On the existence of $L_{\infty \mathrm{K}}$-indiscernibles, 25 (1970), 798-800.

02B25 02C05 02H10

Eldridge, Klaus E.

1 On ring structures determined by groups, 23 (1969), 472-477.

16A46

2 Correction to: On ring structures determined by groups, 25 (1970), 202.

16A46

Elliott, George A.

1 Ideal preserving automorphisms of postliminary $C^{*}$-algebras, 27 (1971), 107-109.

2 A characterization of compact groups, 29 (1971), 621.

$22 \mathrm{C} 05$

Ellis, Robert L.

1 Extending uniformly continuous pseudo-ultrametrics and uniform retracts, 30 (1971), 599-602.

$54 \mathrm{C} 20$ 54E35; 54C15 54F50

Emerson, William R.

1 Groups defined by permutations of a single word, 21 (1969), 386-390.

20F05 32C25 32C30

\section{Engelking, Ryszard}

1 On closed images of the space of irrationals, 21 (1969), 583-586.

54E35

Enochs, Edgar E.

1 (with Coleman, Donald B.) Isomorphic polynomial rings, 27 (1971), 247-252.

$10 \mathrm{M} 05$ 13F20 16A40

Erbe, Lynn H.

1 Oscillation theorems for second order nonlinear differential equations, 24 (1970), 811-814.

$34 \mathrm{C} 15$

Erd8s, Paul

1 (with Stone, A. H.) On the sum of two Borel sets, 25 (1970), 304-306.

04A15 26A21 28A05 54C50

2 (with Kleitman, Daniel J.) On collections of subsets containing no 4-member Boolean algebra, 28 (1971), 87-90.

05A05 05A20

\section{Etgen, Garret J.}

1 Oscillation criteria for nonlinear second order matrix differential equations, 27 (1971), 259-267.

$34 \mathrm{C} 10$

2 (with Scott, J. B.) On the conjugate points of fourth order, selfadjoint linear differential equations, 29 (1971), 349-350.

$34 \mathrm{C} 10$

Etter, Daniel O., Jr.

1 The algebra of log-summable functions, 25 (1970), 1-7.

46E30 46H05

Evans, B. D. 
1 (with Kenelly, John W., Jr.; Hare, William R. and Ludescher, W. H.) Convex components, extreme points, and the convex kernel, 21 (1969), 83-87. $\quad 52$ A30 Evans, E. Graham, Jr.

1 A generalization of Zariski's main theorem, 26 (1970), 45-48.

13B20 14A05 14E05

2 Projective modules as fiber bundles, 27 (1971), 623-626. 16A80 18F25; 13J20 Evans, Trevor

1 (with Dean, Richard A.) A remark on varieties of lattices and semigroups, 21 (1969), 394-396.

\section{Evyatar, Asriel}

06A20 08A15 20M05

1 (with Zaks, Abraham) Purely transcendental subfields of $k\left(x_{1}, \ldots, x_{n}\right), 22(1969)$, 582-586.

12F20 12F99 13F99; 13B99

2 (with Zaks, Abraham) Rings of polynomials, 25 (1970), 559-562.

Fabian, R. J.

$13 \mathrm{~B} 25 ; 13 \mathrm{C} 15$

1 (with Kent, C. F.) Recursive functions defined by ordinal recursions, 23 (1969), 206-210.

Farkas, Hershel M.

1 Abelian differentials with double zeros, 28 (1971), 155-162. 30A52

Faudree, R. J.

1 Locally finite and solvable subgroups of sfields, 22 (1969), 407-413.

16A40 20C05 20E25 20E35; 16A30 16A72 20D10 20E15

2 Groups in which each element commutes with its endomorphic images, 27 (1971), 236-240.

20D15; 20D10 20F45

Fein, Burton

1 Extensions of group representations over fields of prime characteristic, 23 (1969), 11-13.

$20 \mathrm{C} 20$

2 A note on the Brauer-Speiser theorem, 25 (1970), 620-621. 20C15

Ferguson, Edward N.

1 Commutative rims in clans with zero, 23 (1969), 304-305. 22A15; 20M10 Fernholz, E. R.

1 Bounds for holomorphic vector fields, 22 (1969), 344-350.

$32 \mathrm{C} 1532 \mathrm{E} 05$

Feuer, R. D.

1 Torsion-free subgroups of triangle groups, 30 (1971), 235-240. $20 \mathrm{H} 10$ Feustel, C. D.

$1 \mathscr{M}^{3}$ admitting a certain embedding of $P^{2}$ is a pseudo $P^{3}, 26$ (1970), 215-216.

Fiedler, Miroslav

55A99 57C35 57C99; 55A05 55A10 55D15

1 Bounds for the determinant of the sum of hermitian matrices, 30 (1971), 27-31.

Fields, David E.

$15 \mathrm{~A} 15$ 15A 18

1 Zero divisors and nilpotent elements in power series rings, 27 (1971), 427-433.

Fields, K. L.

13A15 13B99; 13E05 13J05

1 Two remarks on the group algebra of a finite group, 30 (1971), 247-248.

Figá-Talamanca, Alessandro

$20 \mathrm{C} 0520 \mathrm{C} 15$

1 Bounded and continuous random Fourier series on noncommutative groups, 22 
(1969), 573-578.

43A15 43A25 43A30

Fillmore, Jay P.

1 Barbier's theorem in the Lobachevski plane, 24 (1970), 705-709.

$50 \mathrm{C} 20$ 53A35

Fink, A. M.

1 (with St. Mary, Donald F.) On an inequality of Nehari, 21 (1969), 640-642.

$34 \mathrm{C} 10$

2 Almost periodicity of the inverse of a fundamental matrix, 27 (1971), 527-528.

Fink, James P.

1 Complete continuity of the inverse of a positive symmetric operator, 25 (1970), 147-150.

47B25; 47A10

Finkelstein, $M$.

1 (with Lebow, Arnold) A note on 'Nth roots of operators', 21 (1969), 250.

Fisher, J. L.

$47 \mathrm{~A} 60$

1 Embedding free algebras in skew fields, 30 (1971), 453-458.

Fitzgerald, Carl $\mathbf{H}$.

16A08 16A40; 16A06

1 Analyticity and continuation of certain functions of two complex variables, 25 (1970), 536-540.

$32 \mathrm{D} 1532 \mathrm{H} 10$

Fitzpatrick, Ben, Jr.

1 (with Transue, William R. R. and Hinrichsen, J. W.) Concerning upper semicontinuous decompositions of irreducible continua., 30 (1971), 157-163.

54F20; 54B 15

2 Concerning upper semicontinuous decompositions of irreducible continua, 30 (1971), 157-163.

54B15 54F15

\section{Flaschka, Hermann}

1 An asymptotic property of the roots of polynomials, 27 (1971), 451-456.

Flatto, Leopold

30A08; 12D10

1 A new proof of the transposition theorem, 24 (1970), 29-31.

$15 \mathrm{~A} 39$

Fleischman, W. M.

1 (with Gulden, Samuel L. and Weston, J. H.) Linearly ordered topological spaces, 24 (1970), 197-203.

54D20 54F05; 06A45 54A25 54D30

Fleming, D. J.

1 (with Jessup, P. G.) Perfect matrix methods, 29 (1971), 319-324.

$40 \mathrm{CO5} 40 \mathrm{D} 20 \mathrm{40H} 05$

Fletcher, Peter

1 (with McCoy, R. A. and Slover, Rebecca Ellen) On boundedly metacompact and boundedly paracompact spaces, 25 (1970), 335-342. 54D15 54D20 54F45

Fleury, Patrick J.

1 Splittings of Hochschild's complex for commutative algebras, 30 (1971), 405-411.

Flory, Volker

$18 \mathrm{G} 35$ 18H20; $20 \mathrm{CO5}$

1 On the Fourier-algebra of a locally compact amenable group, 29 (1971), 603-606.

22D15 22D20 22D25

Foguel, Shaul Reuven

1 Positive operators on $C(X), 22$ (1969), 295-297. 28A30 47A65 54C40 54D30 


\section{Formanek, Edward}

1 A short proof of a theorem of Jennings, 26 (1970), 405-407.

$18 \mathrm{H} 10$ 20J05; $20 \mathrm{~F} 05$

2 The conjugation representation and fusionless extensions, 30 (1971), 73-74.

Fort, Tomlinson

$20 \mathrm{C} 15$ 20D45

1 The nonhomogenous linear difference equation with variable difference interval, 22 (1969), 262-269.

Fossum, Timothy V.

1 Projective representations and induced linear characters, 24 (1970), 106-111.

Fournier, Gilles

$20 \mathrm{C} 15$

1 On a problem of S. Ulam, 29 (1971), 622.

54E40; 54E35

Fox, Charles

1 Solving integral equations by $L$ and $L^{-1}$ operators, 29 (1971), 299-306.

44A15 44H05 45H05

\section{Fraker, Ross}

1 A uniqueness theorem for certain two-point boundary value problems: A correction, 28 (1971), 631-632.

34B15; 34A40

2 (with Bebernes, J. W.) A priori bounds for boundary sets, 29 (1971), 313-318.

Francis, George K.

34B15; 34A40

1 Titus' homotopies of normal curves, 30 (1971), 511-519.

Franke, Charles $\mathbf{H}$.

30A90 57A05 57D10 57D40

1 The Galois correspondence for linear homogeneous difference equations, 21 (1969), 397-401.

12H10 13B05

Franklin, Lawrence $\mathbf{M}$.

1 On the nonexistence of slices for p-adic transformation groups, 27 (1971), 183-185.

Franklin, Stanley P.

57E10

1 On two questions of Moore and Mrowka, 21 (1969), 597-599.

Franks, R. L.

54D30 54D55; 54A25

1 (with Marzec, R. P.) A theorem on mean-value iterations, 30 (1971), 324-326.

$26 \mathrm{~A} 18$ 47H10 54H25; 40A05 65Q05

Fraser, Grant A.

1 (with Horn, Alfred) Congruence relations in direct products, 26 (1970), 390-394.

Fraser, Robert B., Jr.

08A05 08A15

1 A new metric for a metric space, 21 (1969), 755-761. 54E35; 54D05 54E15 French, James A.

1 A characterization of covering dimension for collection wise normal spaces, 25 (1970), 646-649.

54D15 54F45

Fridy, J. A.

1 Properties of absolute summability matrices, 24 (1970), 583-585.

\section{Friedberg, Stephen}

$40 \mathrm{C05} 40 \mathrm{~F} 05$ 
1 The Fourier transform is onto only when the group is finite, 27 (1971), 421-422.

43A25; 42A72

2 Functions which are Fourier-Stieltjes transforms, 28 (1971), 451-452. 43A25

Friedell, J. C.

1 (with Saworotnow, Parfeny P.) Trace-class for an arbitrary $H^{*}$-algebra, 26 (1970), 95-100.

$46 \mathrm{~K} 15$ 47B 10

\section{Friedland, Schmuel}

1 (with Nehari, Zeev) Univalence conditions and Sturm-Liouville eigenvalues, 24 (1970), 595-603.

34B25

Friedman, Neal

1 (with Katz, Melvin L, Jr.) On additive functionals, 21 (1969), 557-561.

\section{Friesen, Donald $\mathrm{K}$.}

1 Products of normal supersolvable subgroups, 30 (1971), 46-48.

$46 \mathrm{E} 15$

Frolik, Zdeněk

1 Stone-Weierstrass theorems for $C(X)$ with the sequential topology, 27 (1971), 486494.

54D20 54H05

Fuller, Kent R.

1 (with Dickson, Spencer E.) Commutative $Q F-1$ Artinian rings are $Q F, 24$ (1970), 667-670.

$13 \mathrm{E} 1016 \mathrm{~A} 36$

Fuller, R. V.

1 Semiuniform spaces and topological homeomorphism groups, 26 (1970), 365-368.

Fulp, Ronald $O$.

$54 \mathrm{C} 35 ; 54 \mathrm{E} 15$ 54H 15

1 Character semigroups of locally compact inverse semigroups, 27 (1971), 613-618.

Fulton, Curtis M.

$22 \mathrm{~A} 15$

1 Ruled surfaces in elliptic space, 24 (1970), 617-620.

$53 \mathrm{~A} 35$

Furuta, Takayuki

1 (with Nakamoto, Ritsuo) Certain numerical radius contraction operators, 29 (1971), 521-524.

47A 10 47A30

Galbraith, A. S.

1 Lower bounds to the zeros of solutions of $y^{\prime \prime}+p(x) y=0,26$ (1970), 111-116.

Galewski, D. E.

$34 \mathrm{C} 10$

1 (with Costich, O. L. and Doyle, Patrick H.) A characterization of punctured open 3-cells, 28 (1971), 295-298.

57A $10 ; 55 \mathrm{~A} 40$

Galvin, Fred

1 (with Horn, Alfred) Operations preserving all equivalence relations, 24 (1970), 521-523.

$08 \mathrm{~A} 05$

Garabedian, Paul R.

1 An unsolvable equation, 25 (1970), 207-208.

35A05 35F05

García Maynez, Adalberto

1 (with Hunt, John) The separation theorem for quasi-closed sets, 27 (1971), 399-404.

54D05 54D10

Garling, D. J. H.

1 Some remarks on injective envelopes, 27 (1971), 503-505.

46B99 54E35

Garnett, John

1 Positive length but zero analytic capacity, 24 (1970), 696-699; Erratum: 26 (1970), 
701.

30A44; 28A75

Gauld, David

1 The canonical Schoenflies theorem, 27 (1971), 603-612.

57A50; 57A45

Gelbaum, Bernard R.

1 Fibre bundles and measure, 21 (1969), 603-607.

55F10; $28 \mathrm{~A} 05$

2 Cantor sets in metric measure spaces, 24 (1970), 341-343.

28A10; 54E35

3 Q-uniform Banach algebras, 24 (1970), 344-353.

$46 \mathrm{HOS}$

4 Tensor products of Banach algebras. II, 25 (1970), 470-474. 46H10 46M05 Gellar, Ralph

1 Operators commuting with a weighted shift, 23 (1969), 538-545.

46H15 47B99; 46A35 46A45

2 Spectrum of $X$ satisfying $0 \leqq X^{p} \leqq X, 30$ (1971), 32-36. $\quad$ 06A70 15A42 15A48 Gentile, Enzo R.

1 A note on injective group rings, 23 (1969), 431-432. 16A26 16A52; 20C05 George, John $\mathbf{H}$.

1 (with Sutton, W. G.) Application of Liapunov theory to boundary value problems, 25 (1970), 666-671.

34B15

2 (with Sehgal, V. M. and Smithson, Raymond E.) Application of Liapunov's direct method to fixed point theorems, 28 (1971), 613-620.

$54 \mathrm{C} 60$ 54H25; 54E35 54E45

Geroch, Robert P.

1 (with Kronheimer, E. H. and McCarty, G. S., Jr.) No topologies characterize differentiability as continuity, 28 (1971), 273-274. 54C05 54C30; 26A27

Gersten, Stephen $M$.

1 On the K-theory of Laurent polynomials, 30 (1971), 223-228.

Geveci, Tunc

13D15 16A54 18F25

1 On differentiability of minimal surfaces at a boundary point, 28 (1971), 213-218.

Gibson, Peter M.

30A72 53A10; 30A30 31A20

1 Conversion of the permanent into the determinant, 27 (1971), 471-476.

Giellis, George R.

15A $15 ; 05 B 20$

1 Trace-class for a Hilbert module, 29 (1971), 63-68. $\quad 46 \mathrm{H} 25$ 46K15; 46C10 Gilbert, Richard C.

1 Symmetric operators with singular spectral functions, 21 (1969), 43-49.

47A45 47B25

2 Symmetric operators with twice continuously differentiable spectral functions, 23 (1969), 347-355.

$47 \mathrm{~A} 20$

Gilfeather, Frank

1 Asymptotic convergence of operators in Hilbert space, 22 (1969), 69-76.

46L99 47B05; 47A50

2 The structure and asymptotic behavior of polynomially compact operators, 25 (1970), 127-134.

47B05; 47A10 47A65

3 (with Chow, T. R.) Functions of direct integrals of operators, 29 (1971), 325-330.

Gillam, J. D. 
1 A five sphere decomposition of $E^{2 n-1}, 24$ (1970), 747-753. 57A15; 57A10 Gilman, Robert

1 Complements to solvable Hall subgroups, 27 (1971), 241-243. 20D10 20D40 Gilmer, Robert W., Jr.

1 A note on generating sets for invertible ideals, 22 (1969), 426-427.

$13 \mathrm{C} 10 ; 13 \mathrm{G} 05$

2 (with Arnold, Jimmy T.) On the contents of polynomials, 24 (1970), 556-562.

13B25 13F20

3 (with Mott, Joe Leonard) An algebraic proof of a theorem of A. Robinson, 29 (1971), 461-466.

14A25; 13L05

Ginsberg, Jonathan I.

1 Generators of $S_{\alpha}, 24$ (1970), 784-787.

30A82; 46E15

2 Completeness of $\{\sin n x+K i \cos n x\}, 29$ (1971), 291-293.

$42 \mathrm{~A} 64$ Gitler, Samuel

1 (with Mahowald, Mark E. and Milgram, R. James) Secondary cohomology operations and complex vector bundles, 22 (1969), 223-229.

55F25 55F40 55G10 55G20; 55G45 57F15

Glaser, Leslie C.

1 Monotone noncompact mappings of $E^{r}$ onto $E^{k}$ for $r \geqq 4$ and $k \geqq 3,23$ (1969), 282-286.

54C10 57A15; 54F15

Glasner, Moses

1 (with Chow, Kwang-nan) Bounded in the mean solutions of $\Delta u=P u$ on Riemannian manifolds, 26 (1970), 261-265.

31B05 31B35; 31C25 31D05 35J20 53B20 53C20

2 Energy-finite solutions of $\Delta u=P u$ and Dirichlet mappings, 29 (1971), 553-556. 30A50 30A60 31B99 35J15; 46J10 53C99

Glover, H. H.

1 (with Becker, James C.) Note on the embedding of manifolds in Euclidean space, 27 (1971), 405-410.

57D40 57D99; 55F25 55G40

2 (with Huneke, John Philip) Some spaces that do not have the common fixed point property, 29 (1971), 190-196.

55C20 57A99

Golan, Jonathan S.

1 Characterization of rings using quasiprojective modules. II, 28 (1971), 337-343.

Goldberg, J. L.

16A42 16A50 16A60; 18G20

1 (with Brown, J. W.) Index-dependent parameters of Laguerre and related polynomial sets, 25 (1970), 852-855.

$33 \mathrm{~A} 65 ; 33 \mathrm{~A} 30$

Goldberg, Samuel Irving

1 Integrability of almost Kaehler manifolds, 21 (1969), 96-100. 53B35 53C15

Goldman, Jerry I.

1 Nodal algebras of dimension $p^{3}, 24$ (1970), 156-160.

17A15 17A25

Goldman, Lawrence

1 Algebraic structure of the manifold of solutions of the $N$-body problem, 25 (1970), 417-422.

$12 \mathrm{H} 05$ 70F 10

Goldstein, Jerome A.

1 Some remarks on infinitesimal generators of analytic semigroups, 22 (1969), 91-93. 
2 An asymptotic property of solutions of wave equations, 23 (1969), 359-363.

Goldstein, Myron

$34 \mathrm{G} 05$ 35B40

1 The converse of Cauchy's theorem for arbitrary Riemann surfaces, 25 (1970), 177 178.

30A88; $31 \mathrm{~A} 25$

2 (with Ow, Wellington $\mathrm{H}$.) On the mean-value property of harmonic functions, 29 (1971), 341-344.

31 A05; 30A31

Gollwitzer, H. E.

1 A note on a functional inequality, 23 (1969), 642-647.

26A84; 26A51

2 Nonoscillation theorems for a nonlinear differential equation, 26 (1970), 78-84.

$34 \mathrm{C} 15$

3 (with Hager, R. A.) The nonexistence of maximum solutions of Volterra integral equations, 26 (1970), 301-304.

45D05 45G99

\section{Gonshor, Harry}

1 Injective hulls of $C^{*}$ algebras. II, 24 (1970), 486-491. 4 46J10 46M10 Goodman, Adolph W.

1 (with Rahman, Qazi Ibadur and Ratti, Jogindar S.) On the zeros of a polynomial and its derivative, 21 (1969), 273-274.

30A08

\section{Goodman, Victor}

1 Quasi-differentiable functions on Banach spaces, 30 (1971), 367-370.

26A96 46G05; 58B10 58C20

Goodrich, Robert K.

1 A Riesz representation theorem, 24 (1970), 629-636.

$46 \mathrm{E} 4046 \mathrm{G} 10$

Goodrick, Richard E.

1 A note on Seifert circles, 21 (1969), 615-617.

$55 \mathrm{~A} 25$

Goodwyn, Lew Wayne

1 Topological entropy bounds measure-theoretic entropy, 23 (1969), 679-688.

Gordon, William B.

$28 \mathrm{~A} 65$

1 On the completeness of Hamiltonian vector fields, 26 (1970), 329-331. 34C35

Goto, Morikuni

1 Products of two one-parameter subgroups, 22 (1969), 554.

22E15

Gottlieb, David

1 (with Breuer, Shlomo) Explicit characterization of spherical curves, 27 (1971), 126-127.

34A05 53A05

2 (with Breuer, Shlomo) Separation of roots and oscillation in ordinary linear differential equations of second order, 29 (1971), 487-493. 34C10

Gowrisankaran, Chandra

1 Radon measures on groups, 25 (1970), 381-384. 22A05 28A70

Grabiner, Sandy

1 The nilpotency of Banach nil algebras, 21 (1969), $510 . \quad 46 \mathrm{H} 05$ Graham, Colin C.

1 Symbolic calculus for algebras of Fourier-Stieltjes transforms, 23 (1969), 311-314.

$43 \mathrm{~A} 25$

$2 M_{0}(G)$ is not a prime L-ideal of measures, 27 (1971), 557-562.

Grätzer, György

43A10 43A70; 22B10 28A10 
1 (with Padmanabhan, R.) On idempotent, commutative, and nonassociative groupoids, 28 (1971), 75-80.

08A05 08A25; 20L05

Gray, William J.

1 (with Roberson, Fred A.) On the near equicontinuity of transformation groups, 23 (1969), 59-63.

$34 \mathrm{~A} 10$ 54H15; 54H20 54H 25

2 (with Vaughan, Loy Ottis, Jr.) The almost fixed point property for hereditarily unicoherent continua, 27 (1971), 381-386. 54C60 54H25; 54F50

\section{Greenfield, Stephen J.}

1 Upper bounds on the dimension of extendibility of submanifolds in $C^{n}, 23$ (1969), 185-189.

$32 \mathrm{~A} 10$ 32C05 58C10; 32E05

\section{Greenleaf, Newcomb}

1 Watts cohomology of field extensions, 21 (1969), 208-210.

$18 \mathrm{H} 20$

2 Fields in which varieties have rational points: A note on a problem of Ax, 27 (1971), 139-140.

12F10; 13L05

Greenlee, W. M.

1 A two parameter perturbation estimate, 24 (1970), 67-74. 35B25 35B30

2 On two parameter singular perturbation of linear boundary value problems, 27 (1971), 268-274.

35B25 47A55; 35J40

Greenwell, D. L.

1 Reconstructing graphs, 30 (1971), 431-433.

$05 C 99$ Greenwood, Priscilla E.

1 An asymptotic estimate of Brownian path variation, 21 (1969), 134-138.

60G17 60J65

Gregorac, Robert J.

1 On residually finite generalized free products, 24 (1970), 553-555.

$20 \mathrm{E} 3020 \mathrm{~F} 15$

Gregory, D. A.

1 (with Shapiro, J. H.) Nonconvex linear topologies with the Hahn Banach extension property, 25 (1970), 902-905.

46A15

\section{Griffith, Phillip A.}

1 Extensions of free groups by torsion groups, 24 (1970), 677-679.

20K10 20K35

2 (with Robson, J. C.) A theorem of Asano and Michler, 24 (1970), 837-838.

Grimm, L. J.

16A12 16A46

1 Existence and continuous dependence for a class of nonlinear neutral-differential equations, 29 (1971), 467-473.

34K05; 34A10

Grimmer, R. C.

1 (with Burton, T. A.) On continuability of solutions of second order differential equations, 29 (1971), 277-283.

$34 \mathrm{~A} 15$ 34C15

Gross, Fletcher

1 p-solvable groups with few automorphism classes of subgroups of order $p, 30$ (1971), 437-444.

20D45

Gross, Jonathan L.

1 An infinite class of irreducible homology 3-spheres, 25 (1970), 173-176.

55A05 55A25 57A10

2 Prime 3-manifolds and the doubling operation, 27 (1971), 375-380. 


\section{Grosshans, Frank}

1 Representations of algebraic groups preserving quaternion skew-hermitian forms, 24 (1970), 497-501.

14L10 16A40

Grove, Larry $C$.

1 Real representations of metacyclic groups, 21 (1969), 417-421. 20C10

2 (with Benson, Clark T.) Generators and relations for Coxeter groups, 24 (1970), 545-547.

Gudder, Stanley P.

1 Quantum probability spaces, 21 (1969), 296-302. 81A12

2 (with Schelp, R. H.) Coordinatization of orthocomplemented and orthomodular posets, 25 (1970), 229-237. 06A10 20M15; 06A25 06A30

3 (with Haskins, L.) Semimodular posets and the Jordan-Dedekind chain condition, 28 (1971), 395-396.

Guggenheimer, Heinrich W.

1 Geometric theory of differential equations. II: Analytic interpretation of a geometric theorem of Blaschke, 29 (1971), 87-90. 34A30 34C10

\section{Gulden, Samuel L.}

1 (with Fleischman, W. M. and Weston, J. H.) Linearly ordered topological spaces, 24 (1970), 197-203.

54D20 54F05; 06A45 54A25 54D30

Gunderson, R. W.

1 A comparison lemma for higher order trajectory derivatives, 27 (1971), 543-548.

\section{Gupta, Narain Datt}

34A40; 34D20

1 The free metabelian group of exponent $p^{2}, 22$ (1969), 375-376. $20 \mathrm{~F} 50$

Gupta, Ram Niwas

1 Nilpotent matrices with invertible transpose, 24 (1970), 572-575.

$15 \mathrm{~A} 33$

Guseman, Lawrence F., Jr.

1 Fixed point theorems for mappings with a contractive iterate at a point, 26 (1970), 615-618.

Gustafson, G. B.

1 The nonequivalence of oscillation and nondisconjugacy, 25 (1970), 254-260.

\section{Gustafson, Karl}

1 The Toeplitz-Hausdorff theorem for linear operators, 25 (1970), 203-204.

\section{Gutiérrez-Novoa, Lino}

1 Independence of a certain axiomatic system, 22 (1969), 470.

Hadlock, E. H.

1 (with Moore, Theral O.) A new definition of a reduced form, 25 (1970), 105-113.

Haeuslein, G. $\mathbf{K}$.

$10 \mathrm{C} 05$

1 On the algebraic independence of symmetric functions, 25 (1971), 178-182.

\section{Hag, Kari}

$12 \mathrm{D} 99$

1 A theorem on T-fractions corresponding to a rational function, 25 (1970), 247-253.

\section{Hagan, Melvin R.}


1 A note on connected and peripherally continuous functions, 26 (1970), 219-223.

54C10; 54D05 54F55

2 Conditions for continuity of certain open monotone functions, 30 (1971), 175-178.

Hager, Anthony W.

54C10 54D05; 54F20

1 Approximation of real continuous functions on Lindelöf spaces, 22 (1969), 156-163.

54C40; 54A25

2 The projective resolution of a compact space, 28 (1971), 262-266.

54D30 54D35; 18B99 54C05 54C10 54C15

Hager, R. A.

1 (with Gollwitzer, H. E.) The nonexistence of maximum solutions of Volterra integral equations, 26 (1970), 301-304. 45D05 45G99

Hagis, Peter, Jr.

1 A root of unity occurring in partition theory, 26 (1970), 579-582. 10A45

Hagopian, Charles L.

1 Mutual aposyndesis, 23 (1969), 615-622.

54F15 54F20; 54B10 54E45

2 A class of arcwise connected continua, 30 (1971), 164-168.

54F20 57A05; 54F25

Hahn, Liang-shin

1 A theorem on multipliers of type ( $p, q), 21$ (1969), 493-495.

43A22

Haimo, Deborah Tepper

1 An integral representation for generalized temperatures in two space variables, 30 (1971), 533-538.

$35 \mathrm{C} 15$ 35K05

Haimo, Franklin

1 Radicals with integrity and row-finite matrices, 24 (1970), 144-147.

Hájek, Otomar

1 Parallelizability revisted, 27 (1971), 77-85. 34C35; 58F10

Hale, Jack K.

1 (with Izé, Antonio F.) On the uniform asymptotic stability of functional differential equations of the neutral type, 28 (1971), 100-106.

$34 \mathrm{~K} 20$

Hales, A. W.

1 Partition representations of free lattices, 24 (1970), 517-520. $\quad$ 06A20; 08A10

Hall, C. E.

1 F-projective objects, 26 (1970), 193-195.

$18 \mathrm{G} 05$ 22A05; 18D35

Hall, Michael H.

1 On the topological classification of the floors of certain Hilbert fundamental domains, 28 (1971), 67-70.

10D05 12A25 32N99; 55D10

Hallam, Thomas G.

1 An asymptotic expansion for a nonhomogeneous linear system, 22 (1969), 489-497.

34E05

2 An asymptotic solution of a nonhomogeneous linear system of differential equations, 29 (1971), 529-534.

$34 \mathrm{D} 05$

Halpern, Benjamin R.

1 The kernel of a starshaped subset of the plane, 23 (1969), 692-696. $\quad 52$ A30

2 Mean convergence and compact subsets of $L_{1}, 28$ (1971), 122-126.

28A20 46E30

3 On the immersion of an n-dimensional manifold in $n+1$-dimensional Euclidean 
space, 30 (1971), 181-184.

57D40

Halpern, James D.

1 (with Howard, Paul E.) Cardinals $m$ such that $2 m=m, 26$ (1970), 487-490.

02K20 04A25

Hammett, Michael E.

1 Nonoscillation properties of a nonlinear differential equation, 30 (1971), 92-96.

34C15 34D05

Hamstrom, Mary-Elizabeth

1 (with Jerrard, R. P.) Collapsing a triangulation of a 'knotted' cell, 21 (1969), 327-331.

55A10 55A25 55C99; 55C10

Hansell, Roger W.

1 General embedding properties of absolute Borel and Souslin spaces, 27 (1971), 343-352.

02K30 04A15 28A05 54D35 54H05; 54E35

Hansen, Wolfhard

1 (with Klee, Victor L.) Intersection theorems for positive sets, 22 (1969), 450-457.

Hanson, T. H. McH.

1 Some semigroups on a manifold with boundary, 25 (1970), 830-835.

22A15 54H15 57E99; 22E15 22E99 57A15

Hare, William R.

1 (with Kenelly, John W., Jr.; Evans, B. D. and Ludescher, W. H.) Convex components, extreme points, and the convex kernel, 21 (1969), 83-87.

52A 30

2 (with Kenelly, John W., Jr.) Sets expressible as unions of two convex sets, 25 (1970), 379-380.

52A05 53A 30

3 (with Stavrakas, Nick M. and Kennelly, John ) Two cells with $N$ points of local nonconvexity, 27 (1971), 331-336.

$52 \mathrm{~A} 10$

Harrell, C. E.

1 Riesz matrices that are also Hausdorff matrices, 22 (1969), 303-304.

Harrison, K. J.

40G05 40G99

1 (with Radjavi, Heydar and Rosenthal, Peter) A transitive medial subspace lattice, 28 (1971), 119-121. 47A15; 47C05

Hartfiel, D. J.

1 A simplified form for nearly reducible and nearly decomposable matrices, 24 (1970), 388-393.

$15 \mathrm{~A} 21$

2 On constructing nearly decomposable matrices, 27 (1971), 222-228.

15A21; 15A15 15A36 15A48

3 Concerning diagonal similarity of irreducible matrices, 30 (1971), 419-425.

Hartman, Philip

15A21 15A30; $15 \mathrm{~A} 60$

1 On disconjugacy criteria, 24 (1970), 374-381.

Hartmann, Frederick

1 Inclusion theorems for Sonnenschein matrices, 21 (1969), 513-519.

Harvey, Charles

40C15 40D25; $40 \mathrm{G} 99$

1 (with Harvey, Reese) Open mappings and the lack of fully completeness of $\mathscr{D}^{\prime}(\Omega)$, 25 (1970), 786-790.

35E10 46A30; 46E35

Harvey, Reese 
1 (with Harvey, Charles) Open mappings and the lack of fully completeness of $\mathscr{Q}^{\prime}(\Omega)$, 25 (1970), 786-790. 35E10 46A30; 46E35

\section{Hasegawa, Minoru}

1 On quasi-analytic vectors for dissipative operators, 29 (1971), 81-84.

47B44; 47D05

\section{Haskins, $\mathbf{L}$.}

1 (with Gudder, Stanley P.) Semimodular posets and the Jordan-Dedekind chain condition, 28 (1971), 395-396.

06A 10 Haslam, H. B.

1 On $H$-spaces mod $\mathfrak{C}_{p}, 30$ (1971), 383-387.

Haslam, Harold B.

1 H-spaces and the suspension homeomorphism, 26 (1970), 689-694.

55D40 55D45 55G45; 55E15

2 A Whitehead type theorem, 29 (1971), 599-602.

55D45 55E05

Hawley, Douglas

1 Compact group topologies for $R, 30$ (1971), 566-572. 22B05; 28A20 28A70

Hayes, Allan

1 (with Davies, Roy O. and Rousseau, George) Complete lattices and the generalized Cantor theorem, 27 (1971), 253-258.

06A10; 06A20

Haynsworth, Emilie V.

1 (with Crabtree, Douglas E.) An identity for the Schur complement of a matrix, 22 (1969), 364-366.

2 Applications of an inequality for the Schur complement, 24 (1970), 512-516.

Headley, V. B.

1 Some oscillation properties of selfadjoint elliptic equations, 25 (1970), 824-829.

Hebert, D. J., Jr.

1 Generalized balayage and a Radon-Nikodym theorem, 26 (1970), 165-167.

Hedlund, James $\mathbf{H}$.

$31 \mathrm{C} 15$ 60G45 60J45; 46A40

1 Multipliers of $H^{1}$ and Hankel matrices, 22 (1969), 20-23. 30A78

Heidel, Jon W.

1 A nonoscillation theorem. for a nonlinear second order differential equation, 22 (1969), 485-488.

$34 \mathrm{C} 15$

Heil, Wolfgang

1 On the existence of incompressible surfaces in certain 3-manifolds, 23 (1969), 704707.

55A25 57A10; 55A05

2 On the existence of incompressible surfaces in certain 3-manifolds. II, 25 (1970), 429-432.

55A05 55A25

Heinicke, A. G.

1 Subdirect sums, hereditary radicals, and structure spaces, 25 (1970), 29-33.

Heinzer, Martin N.

16A21 16A48; 54H10

1 Higher derivations of Wildly ramified v-rings, 23 (1969), 94-100.

Heinzer, William $\mathbf{J}$.

$13 \mathrm{~B} 1013 \mathrm{~B} 15$

1 On Krull overrings of a noetherian domain, 22 (1969), 217-222. 
2 Integral ring extensions and prime ideals of infinite rank, 28 (1970), 344-346.

Helton, Burrell, W.

1 A product integral representation for a Gronwall inequality, 23 (1969), 493-500.

26A39 26A84

2 Solutions of $f(x)=f(a)+(R L) \int_{a}^{x}(f H+f G)$ for rings, 25 (1970), 735-742. 26A39 45A05; 46L99

Henderson, George W.

1 On the hyperspace of subcontinua of an arc-like continuum, 27 (1971), 416-417. 54B20 54C25 54F20 54F50; 57A10

\section{Hengartner, Walter}

1 (with Schober, G. E.) Analytic functions close to mappings convex in one direction, 28 (1971), 519-524.

30A32 30A34

Henry, Myron S.

1 (with Stein, F. Max) An approximate solution of the Riccati matrix equation, 25 (1970), 8-12.

$34 \mathrm{~A} 45$

\section{Hentzel, Irvin Roy}

$1(-1,1)$ rings, 22 (1969), 367-374.

17A30 17D05 17E05

$2(-1,1)$ Algebras, 24 (1970), 24-28.

17A30 17D05 17E05

Hermes, Henry

1 On continuous and measurable selections and the existence of solutions of generalized differential equations, 29 (1971), 535-542. 34A10

Herod, J. V.

1 Multiplicative inverses of solutions for Volterra-Stieltjes integral equations, 22 (1969), 650-656.

45D05

2 A Gronwall inequality for linear Stieltjes integrals, 23 (1969), 34-36.

26A30 26A86

3 A product integral representation for an evolution system, 27 (1971), 549-556.

Herrlich, Horst

47D05 47H15

1 Regular-closed, Urysohn-closed and completely Hausdorff-closed spaces, 26 (1970), 695-698.

54D25 54G20; 54B10

Herzog, Marcel

1 On centralizers of involutions, 22 (1969), 170-174.

$20 \mathrm{D05}$

2 On groups of order $2^{a} 3^{\beta} p^{\gamma}$ with a cyclic Sylow 3-subgroup, 24 (1970), 116-118.

$20 \mathrm{D} 05$

3 On Burnside's lemma, 28 (1971), 379-380.

$20 \mathrm{C} 15$

Hess, Peter

1 A variational approach to a class of nonlinear eigenvalue problems, 29 (1971), 272-276.

47H99; 47H05 49G05

2 On nonlinear equations of Hammerstein type in Banach spaces, 30 (1971), 308-312. 45G99 47A50 47H15

Hethcote, Herbert W.

1 Bounds for zeros of some special functions, 25 (1970), 72-74.

$34 \mathrm{C} 10$

Heyde, C. C.

1 A note concerning behaviour of iterated logarithm type, 23 (1969), 85-90. 


\section{Hickin, Kenneth $\mathbf{K}$.}

1 A class of groups whose local sequence is nonstationary, 21 (1969), 402-408.

\section{Hildebrant, John A.}

20E10 20E15

1 The universal compact subunithetic semigroup, 23 (1969), 220-224.

$22 \mathrm{~A} 15$

Hill, E. T.

1 The annihilator of radical powers in the modular group ring of a p-group, 25 (1970), 811-815.

2 Ideals in the modular group ring of a p-group, 28 (1971), 389-390.

Hill, Paul D.

$20 \mathrm{C05} 20 \mathrm{C} 20$

1 On transitive and fully transitive primary groups, 22 (1969), 414-417. 20K30

2 A summable $C_{\Omega}$-group, 23 (1969), 428-430.

3 Automorphisms of countable primary abelian groups, 25 (1970), 135-140.

$20 \mathrm{~K} 30$

4 A note on extensions of free groups by torsion groups, 27 (1971), 24-28.

Hilliker, D. L.

$20 \mathrm{~K} 1020 \mathrm{~K} 35$

1 (with Straus, Ernst G.) Some p-adic versions of Polya's theorem on integer valued analytic functions, 26 (1970), 395-400.

$12 \mathrm{~B} 05$

Himmelberg, Charles J.

1 (with Porter, Jack R. and Van Vleck, F. S.) Fixed point theorems for condensing multifunctions, 23 (1969), 635-641.

$47 \mathrm{H} 10$

Hindley, Roger

1 (with Lercher, Bruce) A short proof of Curry's normal form theorem, 24 (1970), 808-810.

$02 \mathrm{C} 20$

Hindman, Neil

1 On the existence of $c$-points in $\beta N \backslash N, 21$ (1969), 277-280.

54A25 54D35 54D40; 02K25 04A30

Hinrichsen, J. W.

1 (with Transue, William R. R. and Fitzpatrick, Ben, Jr.) Concerning upper semicontinuous decompositions of irreducible continua., 30 (1971), 157-163.

$54 \mathrm{~F} 20 ; 54 \mathrm{~B} 15$

Hirschfelder, John J.

$1 \mathrm{On} W u$ 's form of the first main theorem of value distribution, 23 (1969), 548-554.

$32 \mathrm{H} 25 ; 53 \mathrm{C} 5558 \mathrm{C} 10$

Hirshon, $\mathbf{R}$.

1 Cancellation of groups with maximal condition, 24 (1969), 401-403. $20 \mathrm{E} 30$ Hochschild, G.

1 Note on algebraic Lie algebras, 29 (1971), 10-16.

17B45 20G15 20G20; 16A24 17B40

\section{Hochstadt, Harry}

1 On an inequality of Lyapunov, 22 (1969), 282-284. 34C10; 34B05

Hochster, $M$.

1 Rings with nondiscrete ideal topologies, 21 (1969), 357-362. 13J99; 13E05

2 Subsemigroups of amenable groups, 21 (1969), 363-364. 20F05 20M05

3 Rings of continuous functions and totally integrally closed rings, 25 (1970), 439-442. 
Hodel, Richard E.

$54 \mathrm{C} 40$ 54G05

1 A note on subparacompact spaces, 25 (1970), 842-845.

$54 \mathrm{D} 20$

Hoffiman, Alan J.

1 On the covering of polyhedra by polyhedra, 23 (1969), 123-126.

Hofimann, Laurence D.

1 Pseudo-uniform convexity of $H^{1}$ in several variables, 26 (1970), 609-614.

30A98 46E 15 ; 41A50

Hofler, John T.

1 Continuous lattice ordering by Schauder basis cones, 30 (1971), 527-532.

46A35 46A40

Holbrook, John A. R.

1 The iterates of a contraction and its adjoint, 29 (1971), 543-546.

Holland, Samuel S., Jr.

47A05; 46C05 47A10

1 An m-orthocomplete orthomodular lattice is m-complete, 24 (1970), 716-718. 06A23 06A25 06A30; 06A40

Holsztyński, W.

1 Approximation by homeomorphisms and solution of $P$. Blass problem on pseudoisotopy, 27 (1971), 598-602.

57A05 57A35; 55A 10 55C25

Holub, J. R.

1 Some problems concerning bases in Banach spaces, 23 (1969), 521-525. 46B15

2 Bases of type $P$ and reflexivity of Banach spaces, 25 (1970), 357-362.

46B10 46B15

3 Integral operators in Banach spaces, 29 (1971), 75-80. 47B10; 46B99

Hooper, R. C.

1 Many topological abelian groups have dense divisible subgroups, 23 (1969), 555-558.

Horn, Alfred

22B05

1 (with Galvin, Fred) Operations preserving all equivalence relations, 24 (1970), 521-523.

$08 \mathrm{~A} 05$

2 (with Fraser, Grant A.) Congruence relations in direct products, 26 (1970), 390-394.

08A05 08A15

Horowitz, S.

1 Strong ergodic theorems for Markov processes, 23 (1969), 328-334.

47A35 47D05 60F99 60J05; 28A70

Hosack, John M.

1 The spectrum of a selfadjoint compression of a selfadjoint operator, 25 (1970), 83-85.

47A10 47A20

2 Semicontinuity of nullity or deficiency implies normability of the space, 30 (1971), 321-323.

46A05 47B30

Howard, F. T.

1 A property of the Rayleigh function, 24 (1970), 719-723.

$10 \mathrm{~A} 40$

2 A combinatorial problem and congruences for the Rayleigh function, 26 (1970), 574-578.

05A15 10A10 10A20

3 The number of binomial coefficients divisible by a fixed power of 2, 29 (1971), 236-242.

05A10; 10A25 
Howard, Paul E.

1 (with Halpern, James D.) Cardinals $m$ such that $2 m=m, 26$ (1970), 487-490. $02 \mathrm{~K} 20$ 04A25

Howland, James S.

1 Analytic continuation of the S-matrix for potential scattering, 21 (1969), 381-385.

2 Analyticity of determinants of operators on a Banach space, 28 (1971), 177-180. 46B99 46F15; 46M05 47A55

Hubbuck, John

1 Associative $H$-spaces with small ranks, 30 (1971), 375-382.

55D35 55D45 55F40 55G10; 57F05

Huckaba, James A.

1 A class of non-noetherian domains, 24 (1970), 659-666.

$13 E 99$

Hudson, Sigmund N.

1 On connectivity properties of finite-dimensional groups, 23 (1969), 68-72.

Huff, B. W.

22A05 22D05

1 Comments on the continuity of distribution functions obtained by superposition, 27 (1971), 141-146.

60E05; 60B10 60J10

Huff, Robert E.

1 Common eigenvectors for commutative positive linear operators, 25 (1970), 51-55.

Hughes, David K.

47B55

1 Linear differential-difference operators and their adjoints, 26 (1970), 408-414.

Hughes, Robert B.

34B05 34J10

1 Zero sets of functions from non-quasi-analytic classes, 27 (1971), 539-542.

Hummel, James A. ,

26A93; 35K05

1 The coefficients of starlike functions, 22 (1969), 311-315.

30A32 30A34 Huneke, John Philip

1 Extending commuting functions, 24 (1970), 206-208. 26A15 26A18 26A30

2 (with Glover, H. H.) Some spaces that do not have the common fixed point property, 29 (1971), 190-196.

55C20 57A99

Hunt, John

1 (with García Máynez, Adalberto) The separation theorem for quasi-closed sets, 27 (1971), 399-404.

54D05 54D10

Hunt, L. R.

1 Real-analytic submanifolds of complex manifolds, 29 (1971), 69-74.

32C05 32D10; 32E10

Huq, Syed A.

1 An interpolation theorem for adjoint functors, 25 (1970), 880-883.

15A69 18B10; 08A10 15A72 18C05 18C10

Hursey, R. J., Jr.

1 On ordered polycyclic groups, 28 (1971), 391-394.

06A20 06A55 06A55 20B25 20B25; 20E15 20E25

Husch, Lawrence S.

1 Finding a boundary for a 3-manifold, 21 (1969), 64-68. 
55A05 57A10; 55A35

2 Unknotting in codimension one, 23 (1969), 215-219.

57C35 57C99 57D80; 55A05 57C45

3 A topological characterization of the dilation in $E^{n}, 28$ (1971), 234-236.

Hutchinson, John J.

1 Quotient full linear rings, 28 (1971), 375-378. 16A08 16A42

Hyman, D. M.

1 A note on closed maps and metrizability, 21 (1969), 109-112. 54E35; 54C10 Inagaki, Nobuo

1 On $\mathfrak{F}$-normalizers and $\mathfrak{F}$-hypercenter, 26 (1970), 21-22. 20D10 20D20

Ingraham, Edward C.

1 (with Brown, William C.) A characterization of semilocal inertial coefficient rings, 26 (1970), 10-14.

13H99 13J15 16A16

Insley, R. B.

1 (with Riddell, R. C.) Commuting operator solutions of algebraic equations, 28 (1971), 461-463.

47A50

Isaacs, G. L.

1 A limitation theorem for absolute summability, 29 (1971), 47-54.

40F05 40G05; 40C10 40D99

Isaacs, I. M.

1 Groups having at most three irreducible character degrees, 21 (1969), 185-188. 20C15 20D10

2 Degrees of sums in a separable field extension, 25 (1970), 638-641. $\quad 12 \mathrm{~F} 10$ Isbell, John R.

$1 \mathrm{~s}$ admits an injective metric, 28 (1971), 259-261.

54E35 54E40; 57A 17

Istrăţescu, Ioana

1 (with Constantin, Gheorghe) Some remarks on structure of Riesz operators, 21 (1969), 455-458.

47A65 47B05; 47B20

Itô, Takashi

1 Multiplicative properties of Jensen measures, 26 (1970), 305-306. 46J10 Iwata, Kôichi

1 Span of lens spaces, 26 (1970), 687-688. 55G40 55H25 57D25

Iyengar, H. R. Krishna

1 Semilattice of bisimple regular semigroups, 28 (1971), 361-365. 20M10 Izé, Antonio F.

1 On an asymptotic property of a Volterra integral equation, 28 (1971), 93-99.

45D05 45G99 45M05

2 (with Hale, Jack K.) On the uniform asymptotic stability of functional differential equations of the neutral type, 28 (1971), 100-106.

$34 \mathrm{~K} 20$ Jackson, Robert E.

1 Quasi-Jordanian continua, 27 (1971), 387-390.

54F20; 54E30 57A05 Jacobinski, $\mathbf{H}$.

1 On embedding of lattices belonging to the same genus, 24 (1970), 134-136.

2 Two remarks about hereditary orders, 28 (1971), 1-8. 16A18; 13F05 16A60 Jain, N. C.

1 (with Kallianpur, Gopinath) Norm convergent expansions for Gaussian processes in 
Banach spaces, 25 (1970), 890-895.

20D15 20D45 28A40 46B15 60B05 60F15 60G15 60G20; 46A05 46B05

2 A zero-one law for Gaussian processes, 29 (1971), 585-587.

60F20 60G15 60G20 60G30; 46E10 62M15

Jain, S. K.

1 (with Bhattacharya, P. B.) Rings having solvable adjoint groups, 25 (1970), 563-565.

\section{Jakimovski, Amnon}

1 (with Skerry, Herbert) Some regularity conditions for the $\left(f, d_{n}, z_{1}\right)$ summability method, 24 (1970), 281-287. 40C05 40D05 40G99 James, D. G.

1 Indefinite quadratic forms of determinant $\pm 2 p, 21$ (1969), 214-218.

$10 \mathrm{C05} 15 \mathrm{~A} 63$

James, Ioan M.

1 On the homotopy type of Stiefel manifolds, 29 (1971), 151-158.

55D15 55F10 55F99 55G10 55G40 57F20; 55E15 55F20 55G20 57F15

Jamison, Benton

1 Irreducible Markov operators on $C(S), 24$ (1970), 366-370.

2 (with Orey, Steven) Subgroups of sequences and paths, 24 (1970), 739-744.

Janoš, Ludvík 22A99 28A35 28A40 43A05 60F20 60G15 60G30; 60G17 62E10

1 Topological homotheties on compact Hausdorff spaces, 21 (1969), 562-568.

54C10 54D30 54E40; 54E15 54E45 54H25

2 On representations of selfmappings, 26 (1970), 529-533.

$54 \mathrm{C} 05$ 54H15; 22A 15 54D30

3 On maximal groups of isometries, 28 (1971), 584-586. 54E35 54H15; 54E45 Jaworowski, Jan W.

1 A fixed point theorem for manifolds, 28 (1971), 275-278.

54C10 54C55 54H25 55C15 55C20 57A15; 57A10

Jenkins, James A.

1 A uniqueness result in conformal mapping, 22 (1969), 324-325. 30A30 Jenkins, Joe W.

1 Amenable subsemigroups of a locally compact group, 25 (1970), 766-770.

2 Subsemigroups of an amenable group, 26 (1970), 226-227.

3 Sigma-amenable locally compact groups, 28 (1971), 621-626.

Jenkins, Terry L.

22A20 22D05; 20M05 20M99

1 (with Kreiling, Daryl) Semisimple classes and upper-type radical classes of narings, 26 (1970), 378-382.

16A21 17D05; 17E05 Jensen, G. A.

1 A note on complete separation in the Stone topology, 21 (1969), 113-116.

Jerome, Joseph W.

$06 \mathrm{~A} 2354 \mathrm{C} 40$

1 (with Schumaker, Larry L.) Applications of E-entropy to the computation of $n$ widths, 22 (1969), 719-722.

41A45 46B99

Jerrard, R. P. 
1 (with Hamstrom, Mary-Elizabeth) Collapsing a triangulation of a 'knotted' cell, 21 (1969), 327-331.

55A10 55A25 55C99; 55C10 Jessup, $\mathbf{P}$. G.

1 (with Fleming, D. J.) Perfect matrix methods, 29 (1971), 319-324.

40C05 40D20 40H05

Jobe, John

1 The intersection of indecomposable continua, 23 (1969), 623-624.

54E30 54F20; 54F15

2 (with Crawford, Albert L.) A characterization of hereditarily indecomposable continua, 27 (1971), 205-208. 54E30 54F20; 54D05 54E35 Jockusch, Carl G., Jr.

1 (with Soare, Robert I.) Minimal covers and arithmetical sets, 25 (1970), 856-859.

Jodeit, Max, Jr.

1 A note on Fourier multipliers, 27 (1971), 423-424. Joffe, A. D.

1 On a sequence of almost deterministic pairwise independent random variables, 29 (1971), 381-382.

\section{Johnson, Dudley Paul}

22C05 28A70 43A05 43A75 60A05 60G50; 22D45 43A40 60B15

1 Markov process representations of general stochastic processes, 24 (1970), 735-738.

Johnson, E. W.

60G05 60J35; 46A05 46B99 46G10

1 (with Lediaev, John P.) A new characterization of Dedekind domains, 28 (1971), 63-64. 13F05; 13A15 13E05 13H99 Johnson, Gerald W.

1 Integral representation of multiplicative, involution preserving operators in $\mathscr{L}(C(S)$, E), 23 (1969), 373-377.

$46 \mathrm{G} 1046 \mathrm{H} 15$

2 (with Skoug, D. L.) Operator-valued Feynman integrals of certain finite-dimensional functionals, 24 (1970), 774-780.

$28 \mathrm{~A} 40$

3 (with Skoug, D. L.) An operator valued function space integral: a sequel to Cameron and Storvick's paper, 27 (1971), 514-518. 28A40 Johnson, Gordon G.

1 A crinkled arc, 25 (1970), 375-376. 46C05

Johnson, J. A.

1 Quotients in Noetherian lattice modules, 28 (1971), 71-74. 06A30; 13C05

Johnson, Norman Lloyd

$1 A$ note on the construction of quasifields, 29 (1971), 138-142.

Johnson, R. E.

50D05; 05B25 25B25

1 Extended Malcev domains, 21 (1969), 211-213.

06A50 20M15

2 (with Beauregard, Raymond A.) Primary factorization in a weak Bezout domain, 25 (1970), 662-665.

16A02; $13 \mathrm{G} 05$

3 Unique factorization monoids and domains, 28 (1971), 397-404.

\section{Johnson, Roy A.}

16A02 20M25; 06A50

1 Some types of Borel measures, 22 (1969), 94-99.

28A 10

2 Atomic and nonatomic measures, 25 (1970), 650-655.

28A 10 
Johnson, W. B.

1 (with Dyer, James A.) Isomorphisms generated by fundamental and total sets, 22 (1969), 330-334.

2 No infinite dimensional P space admits a Markuschevich basis, 26 (1970), 467-468.

Jondrup, S.

$46 \mathrm{~B} 15$

1 p.p. rings and finitely generated flat ideals, 28 (1971), 431-435.

Jones, Gary D.

13C10 16A50; 13F20

1 An embedding theorem for homeomorphisms of the closed disc, 26 (1970), 352-354.

54H 20; 54H15 57E25

Jones, John P.

1 Independent recursive axiomatizability in arithmetic, 23 (1969), 107-113.

Jones, Lee

$02 \mathrm{G} 10$

1 (with Kuftinec, Velimir) A note on the Blum-Hanson theorem, 30 (1971), 202-203. 28A65 47A20 47A35

Jonker, $\mathbf{L}$.

i A iote on a paper of Palais, 27 (1971), 337-340.

58A05 58A 10 Jorgensen, Murray

1 An equivalent form of Lév'y's axiom schema, 26 (1970), 651-654.

02K35 04A 10

Kaczynski, T. J.

1 The set of curvilinear convergence of a continuous function defined in the interior of a cube, 23 (1969), 323-327.

26A15 26A54

Kadison, Richard V.

1 (with Aarnes, Johan F.) Pure states and approximate identities, 21 (1969), 749-752.

46L05

Kagiwada, Harriet $\mathbf{H}$.

1 (with Kalaba, Robert E. and Schumitzky, Alan) A representation for the solution of Fredholm integral equations, 23 (1969), 37-40.

45B05

Kahn, Donald W.

1 A note on stable homotopy modules, 26 (1970), 683-686.

Kalaba, Robert E.

1 (with Kagiwada, Harriet $\mathrm{H}$. and Schumitzky, Alan) A representation for the solution of Fredholm integral equations, 23 (1969), 37-40.

45B05

\section{Kallianpur, Gopinath}

1 (with Jain, N. C.) Norm convergent expansions for Gaussian processes in Banach spaces, 25 (1970), 890-895.

20D15 20D45 28A40 46B15 60B05 60F15 60G15 60G20; 46A05 46B05

$60 \mathrm{G} 17$

\section{Kallman, Robert R.}

1 The strong-bounded topology on groups of automorphisms of a von Neumann algebra, 23 (1969), 367-372.

$46 \mathrm{~L} 10$

2 One-parameter groups of *-Automorphisms of II von Neumann algebras, 24 (1970), 336-340.

$46 \mathrm{~L} 10$

Kalmbach, Gudrun 
1 On smooth bounded manifolds, 22 (1969), 466-469.

$32 \mathrm{E} 1058 \mathrm{E} 05$

Kalton, N. J.

1 A barrelled space without a basis, 26 (1970), 465-466.

46A35

Kantorovitz, Shmuel

1 On the operational calculus for groups of operators, 26 (1970), 603-608.

Karcher, Hermann

1 A short proof of Berger's curvature tensor estimates, 26 (1970), 642-644.

$47 \mathrm{~A} 60$ 47D10

Karrass, Abraham

1 (with Solitar, Donald) On finitely generated subgroups of a free group, 22 (1969), $\begin{array}{ll}209-213 . & \text { 20E05 }\end{array}$

2 (with Solitar, Donald) On groups with one defining relation having an abelian normal subgroup, 23 (1969), 5-10. 20K05

3 (with Baumslag, Gilbert and Solitar, Donald) Torsion-free groups and amalgamated $\begin{array}{ll}\text { products, } 24 \text { (1970), 688-690. 20E30 } & \end{array}$

4 (with Solitar, Donald) On the free product of two groups with an amalgamated subgroup of finite index in each factor, 26 (1970), 28-32. 20E05 20E30

Kartsatos, A. G.

1 Oscillation of nonlinear systems of matrix differential equations, 30 (1971), 97-101.

$34 \mathrm{C} 15$

Kascic, Michael J., Jr.

1 (with Roth, B.) A closed subspace of $\mathscr{L}(\Omega)$ which is not an LF-space, 24 (1970), 801-802.

35E99 46A15

Kass, Seymour

1 (with Witthoft, William G.) Irreducible polynomial identities in anticommutative algebras, 26 (1970), 1-9.

17A05 17A30; 17A10

Kato, Tosio

1 A characterization of holomorphic semigroups, 25 (1970), 495-498. 47D05

Katz, I. Norman

1 Asymptotic behavior of solutions to some nth order linear differential equations, 21 (1969), 657-662.

34D05

Katz, Melvin L, Jr.

1 (with Friedman, Neal) On additive functionals, 21 (1969), 557-561. 46E15 Kauffman, Robert M.

1 Completely continuous inverses of ordinary differential operators, 22 (1969), 657 659.

47E05; 47B05

2 Unitary groups and differential operators, 30 (1971), 102-106.

\section{Kaufman, Robert P.}

34B25 47E05; 47D10

1 Representation of linear sets as critical sets, 25 (1970), 884-889.

Kaul, S. K.

26A16 26A24 26A30 26A45 28A75; 26A33 28A75

1 On pointwise periodic transformation groups, 27 (1971), 391-394.

54H15 54H20; 57A15

\section{Kazdan, Jerry L.}

1 Perturbation of complete orthonormal sets and eigenfunction expansions, 27 (1971), 506-510. 


\section{Ke, William Oo Kian}

1 (with Leighton, Walter) A comparison theorem, 28 (1970), 185-188. $34 \mathrm{C} 10$ Keane, Michael

1 Contractibility of the automorphism group of a nonatomic measure space, 26 (1970), 420-422.

47D 10

Keesling, James Edgar

1 Normality and properties related to compactness in hyperspaces, 24 (1970), 760-766. 54B20; 54D15 54D20

2 Proper mappings and dimension, 29 (1971), 202-204. 54F45; 54C10 54D45

3 Locally compact full homeomorphism groups are zero-dimensional, 29 (1971), 390396.

57E05 57E10; 54C35

4 Proper mappings and the minimum dimension of a compactification of a space, 30 (1971), 593-598.

54C10 54D35 54F45

Kellogg, C. $\mathbf{N}$.

1 Pseudo-uniform convexity in $H^{1}, 23$ (1969), 190-192.

$46 \mathrm{E} 15$

Kelly, Patrick H.

1 (with Larsen, Max D.) Valuation rings with zero divisors, 30 (1971), 426-430.

$13 \mathrm{~A} 15$

Kemperman, J. H. B.

1 (with Maharam, Dorothy) $R^{c}$ is not almost Lindelöf, 24 (1970), 772-773.

Kenelly, John W., Jr.

28A10 54D20

1 (with Hare, William R.; Evans, B. D. and Ludescher, W. H.) Convex components, extreme points, and the convex kernel, 21 (1969), 83-87.

$52 \mathrm{~A} 30$

2 (with Hare, William R.) Sets expressible as unions of two convex sets, 25 (1970), 379-380.

52A05 53A30

3 (with Stavrakas, Nick M. and Hare, William R.) Two cells with $N$ points of local nonconvexity, 27 (1971), 331-336.

Kennebeck, D. R.

1 (with Wright, Fred M. and Klasi, M. L.) The Gronwall inequality for weighted integrals, 30 (1971), 504-510.

26A42 26A86; 34A10 34H05

Kent, C. F.

1 Reducing ordinal recursion, 22 (1969), 690-696.

$02 \mathrm{~F} 27$

2 (with Fabian, R. J.) Recursive functions defined by ordinal recursions, 23 (1969), 206-210.

$02 \mathrm{~F} 27$

Keogh, F. R.

1 (with Başgöze, Türkân) The Hardy class of a spiral-like function and its derivative, 26 (1970), 266-269. 30A30 30A32; 30A78

2 (with Miller, Sanford S.) On the coefficients of Bazilevič functions, 30 (1971), 492-496.

Kerr, Donald R., Jr.

30A32 30A34

1 (with Brown, Herbert I. and Stratton, Howard H., Jr.) The structure of $B[c]$ and extensions of the concept of conull matrix, 22 (1969), 7-14. 46L20; 40H05

Keynes, Harvey B.

1 Lifting of topological entropy, 24 (1970), 440-445.

Khanna, I. $\mathbf{K}$.

54H20; 54H10 
1 (with Agrawal, Bhagwan Das) A general differential equation for classical polynomials, 22 (1969), 646-649.

33A45 33A65

Kibler, Dennis

1 Isotopy and homeomorphism, 26 (1970), 499-502.

$54 \mathrm{C} 1054 \mathrm{C} 2557 \mathrm{C} 05$ Kiernan, Peter

1 Hyperbolic submanifolds of complex projective space, 22 (1969), 603-606.

$32 \mathrm{C} 2532 \mathrm{H} 15$

2 Some results concerning hyperbolic manifolds, 25 (1970), 588-592. 32H 20 Kiltinen, John $\mathbf{O}$.

1 Inverses of polynomial functions in topological fields, 24 (1970), 9-17. $12 \mathrm{~J} 99$ Kim, Woo Jong

1 On a theorem of Pokornyi, 23 (1969), 343-346.

$34 \mathrm{C} 10$

2 Oscillatory properties of linear third-order differential equations, 26 (1970), 286-293.

$34 \mathrm{C} 10$

3 Simple zeros of solutions of nth-order linear differential equations, 28 (1971), 557 561.

$34 \mathrm{C} 10$

Kirk, W. A.

1 Remarks on pseudo-contractive mappings, 25 (1970), 820-823. 47H10

2 A fixed point theorem for mappings with a nonexpansive iterate, 29 (1971), 294-298.

Klasi, M. L.

$47 \mathrm{H} 10$

1 (with Wright, Fred M. and Kennebeck, D. R.) The Gronwall inequality for weighted integrals, 30 (1971), 504-510.

26A42 26A86; 34A10 34H05

Klee, Victor $\mathbf{L}$.

1 (with Hansen, Wolfhard) Intersection theorems for positive sets, 22 (1969), 450-457.

52A20 52A35

Kleiman, Steven $\mathbf{L}$.

1 Ample vector bundles on algebraic surfaces, 21 (1969), 673-676. 14F05; 55F25 Klein, Aaron

1 On categories of quotients, 30 (1971), 205-211.

18A99; 18A20 18E99

Klein, Abraham

1 Three sets of conditions on rings, 25 (1970), 393-398. 16A06 16A22 16A48 Kleinberg, E. M.

1 Weak partition properties for infinite cardinals. I, 30 (1971), 371-374.

Kleinfeld, Erwin

04A20; 02K35

1 (with Kleinfeld, Margaret Humm) A nonidentity for right alternative rings, 22 (1969), 109-110.

17D05

Kleinfeld, Margaret Humm

1 (with Kleinfeld, Erwin) A nonidentity for right alternative rings, 22 (1969), 109-110.

Kleitman, Daniel J.

$17 \mathrm{D} 05$

1 On Dedekind's problem: the number of monotone Boolean functions, 21 (1969), 677-682.

$05 \mathrm{C} 30$ 06A35

2 (with Rothschild, B. L) The number of finite topologies, 25 (1970), 276-282.

05B30; 54A05

3 (with ErdôS, Paul) On collections of subsets containing no 4-member Boolean algebra, 28 (1971), 87-90.

05A05 05A20 
Knighten, Carol M.

1 Some consequences of dim proj $\Omega(A)<\infty, 28$ (1971), 411-414.

13H10 13H99 14B99; 13D99

\section{Knopfmacher, J.}

1 (with Sinclair, Allan M.) Fields with few extensions, 29 (1971), 255-258.

Knus, Max-Albert

1 (with Barr, Michael) Extensions of derivations, 28 (1971), 313-314. $\quad 16$ A72 Knutson, Gerhard W.

1 A characterization of closed 3-manifolds with spines containing no wild arcs, 21 (1969), 483-489.

57A10; 57A05

\section{Koehler, Anne}

1 Quasi-projective covers and direct sums, 24 (1970), 655-658.

\section{Koehler, Donald O.}

16A50; 16A46 16A52

1 A note on some operator theory in certain semi-inner-product spaces, 30 (1971), 363-366.

46B99 47A10 47B99

\section{Koh, Kwangil}

1 On almost maximal right ideals, 25 (1970), 266-272.

16A66; 16A12 16A30 16A40 16A46

2 (with Luh, Jiang) On a finite dimensional quasi-simple module, 25 (1970), 801-807. 16A64; 16A12 16A42

3 On one sided ideals of a prime type, 28 (1971), 321-329.

\section{Kohls, Carl W.}

16A30 16A46 16A66; 16A48

1 Representation of abelian groups and rings by families of real-valued functions, 25 (1970), 86-92.

06A60 06A70 20K20

2 Extensions of a ring by a ring with a bimodule structure, 25 (1970), 846-851.

Kohn, S.

13B99 16A56; 13G05

1 (with Newman, Donald J.) Multiplication from other operations, 27 (1971), 244246.

12E99; 05A05

König, Heinz

1 On the Gleason and Harnack metrics for uniform algebras, 22 (1969), 100-101.

Kost, Frank

$46 \mathrm{~J} 10$

1 Wallman-type compactifications and products, 29 (1971), 607-612.

\section{Kotzen, Marshall}

54B10 54D35; 54C25 54C50

1 On the structure of a finite solvable K-group, 27 (1971), 16-18. 20D10 20D30 Kozlowski, George

1 Factorization of certain maps up to homotopy, 21 (1969), 88-92.

54B15 54C10 57B05; 54D05

Kra, Irwin

1 A generalization of a theorem of Poincaré, 27 (1971), 299-302. 30A58; 30A46 Kraines, David

1 Rational cohomology operations and Massey products, 22 (1969), 238-241. 
Krajkiewicz, P.

$55 \mathrm{G} 2055 \mathrm{G} 3055 \mathrm{G} 45$

1 (with Bosch, W.) Polyanalytic functions with equal modulus, 23 (1969), 127-132.

30A94

2 (with Bosch, W.) The big Picard theorem for polyanalytic functions, 26 (1970), 145-150.

30A96; 30A02 30A70

Krause, Eugene F.

1 (with Weston, Kenneth W.) On the Lie algebra of a Burnside group of exponent 5 , 27 (1971), 463-470. 20F40 20F45; 17B99 20E10

Kraut, Edgar A.

1 On equations of the Wiener-Hopf type in several complex variables, 23 (1969), 24-26.

45E $10 ; 32 \mathrm{~A} 07$

Kreiling, Daryl

1 (with Jenkins, Terry L.) Semisimple classes and upper-type radical classes of narings, 26 (1970), 378-382. 16A21 17D05; 17E05

Kreith, Kurt

1 Sturmian theorems for hyperbolic equations, 22 (1969), 277-281.

35B05 35L05

2 A comparison theorem for conjugate points of general selfadjoint differential equations, 25 (1970), 656-661.

$34 \mathrm{C} 10$

3 (with Benson, Donald C.) On abstract Pruefer transformations, 26 (1970), 137-140.

$34 \mathrm{~A} 05$ 34J05

4 Oscillation criteria for nonlinear matrix differential equations, 26 (1970), 270-272.

$34 \mathrm{C} 1034 \mathrm{C} 15$

5 A class of comparison theorems for nonselfadjoint elliptic equations, 29 (1971), 547-552.

$35 \mathrm{BO} 35 \mathrm{~J} 15$

Kripke, Bernard R.

1 Finitely generated coherent analytic sheaves, 21 (1969), 530-534.

$32 \mathrm{C} 35$

Krishnasastry, M. S. R.

1 A model of Euclidean 2-space, 28 (1971), 114-118.

30A92; 30A96

Kritt, B.

1 Generalized pseudo-hermitian operators, 30 (1971), 343-348.

47A60 47A65 47B15 47B40; 46F99

Kronheimer, E. H.

1 (with Geroch, Robert P. and McCarty, G. S., Jr.) No topologies characterize differentiability as continuity, 28 (1971), 273-274.

54C05 54C30; 26A27

Kronk, Hudson V.

1 Generalization of a theorem of Pósa, 21 (1969), 77-78.

$05 C 35$

Krueger, Warren $M$.

1 Higher order homology operations and the Adams spectral sequence, 27 (1971), 395-398.

55E45 55G05 55G10 55G20 55H15; 55B20

\section{Kruskal, Joseph B.}

1 Two convex counterexamples: A discontinuous envelope function and a nondifferentiable nearest-point mapping, 23 (1969), 697-703. 41A50 46C05 52A20

Ku, Hsu-tung

1 (with $\mathrm{Ku}, \mathrm{Mei}-\mathrm{chin}) \mathrm{On}$ the nonexistence of free differentiable $S^{3}$-actions on homotopy spheres, 21 (1969), 101-103.

57D60 57E15

2 (with $\mathrm{Ku}, \mathrm{Mei}-\mathrm{chin}) \quad A$ note on the index of a G-manifold, 22 (1969), 600-602. 
3 A note on semifree actions of $S^{1}$ on homotopy spheres, 22 (1969), 614-617.

4 (with Ku, Mei-chin) Free differentiable actions of $S^{1}$ and $S^{3}$ on homotopy spheres, 25 (1970), 864-869.

57D20 57D60 57D90 57E15 57E25

Ku, Mei-chin

1 (with $\mathrm{Ku}$, Hsu-tung) On the nonexistence of free differentiable $\mathrm{S}^{3}$-actions on homotopy spheres, 21 (1969), 101-103.

57D60 57E15

2 (with Ku, Hsu-tung) $A$ note on the index of a G-manifold, 22 (1969), 600-602. 55C35 57D20 57E15

3 (with Ku, Hsu-tung) Free differentiable actions of $S^{1}$ and $S^{3}$ on homotopy spheres; 25 (1970), 864-869.

57D20 57D60 57D90 57E15 57E25

Kuczkowski, Joseph E.

1 On roots and subsemigroups of nilpotent groups, 28 (1971), 50-52.

Kuelbs, J. D.

20E15 20M10

1 Expansions of vectors in a Banach space related to Gaussian measures, 27 (1971), 364-370.

Kuftinec, Velimir 28A40 46C10 47B10 60G15 60G17; 47A70 62M10 62M15

1 (with Jones, Lee) A note on the Blum-Hanson theorem, 30 (1971), 202-203.

Kugler, Lawrence D.

1 Nonstandard almost periodic functions on a group, 22 (1969), 527-533.

\section{Kullman, David E.}

1 Developable spaces and p-spaces, 27 (1971), 154-160.

Kulshreshtha, S. K.

54D20 54E20 54E30; 54D35 54E35

1 (with Arora, Kasturi L.) An infinite integral involving Meijer G-function, 26 (1970), 121-125. 30A86 33A35 44A45; 33A15 33A30

\section{Kunz, Ernst}

1 The value-semigroup of a one-dimensional Gorenstein ring, 25 (1970), 748-751.

\section{Kwee, Bun-kung}

$13 \mathrm{H} 10 ; 13 \mathrm{~A} 1514 \mathrm{M} 10$

1 The Hausdorff summability of Fourier series, 24 (1970), 586-592. $\quad 42$ A24

Kwun, Kyung Whan

1 Nonexistence of orientation reversing involutions, on some manifolds, 23 (1969), 725-726.

$55 C 3555 C 99$

Lacey, H. Elton

1 (with Morris, Peter D.) On spaces of type $A(K)$ and their duals, 23 (1969), 151-157.

46E05

2 A note concerning $A^{*}=L_{1}(\mu), 29$ (1971), 525-528. 46E30; 46E15 Lacher, R. C.

1 Suspending homotopy 3-spheres and embedding mapping cylinders in $S^{4}, 27$ (1971), 584-586.

55A40 55A99 57A15; 57A35 57A60

Lachlan, A. H. 
1 (with Madison, Eugene W.) Computable fields and arithmetically definable ordered fields, 24 (1970), 803-807.

12J15 12L99

\section{Ladas, Gerasimos}

1 (with Lakshmikantham, V. and Leela, S.) On the perturbability of the asymptotic manifold of a perturbed system of differential equations, 27 (1971), 65-71.

\section{Lahiri, Maya}

34D05 34E 10

1 On a generalisation of Hermite polynomials, 27 (1971), 117-121.

Lakser, $\mathbf{H}$.

1 Injective hulls of Stone algebras, 24 (1970), 524-529.

Lakshmikantham, $\mathbf{V}$.

1 (with Ladas, Gerasimos and Leela, S.) On the perturbability of the asymptotic manifold of a perturbed system of differential equations, 27 (1971), 65-71.

Lallement, Gérard

34D05 34E 10

1 On a theorem of Malcev, 30 (1971), 49-54.

20M05 20M10

Lam, Kee Yuen

1 Cup product in projective spaces, 24 (1970), 832-833. 55-02 55B45 57F15

Lam, Tsit-yuen

1 (with Reiner, Irving) Finite generation of Grothendieck rings relative to cyclic subgroups, 23 (1969), 481-489.

$20 \mathrm{C05}$

Lambert, Alan

1 Strictly cyclic weighted shifts, 29 (1971), 331-336. $\quad$ 46L15 47B20; 47C05

Lambert, H. W.

1 Replacing certain maps of 3-manifolds by homeomorphisms, 23 (1969), 676-678.

Lancaster, G. M.

57A10; 54C05

1 A characterization of certain conformally Euclidean spaces of class one, 21 (1969), 623-628.

53B25

Landau, Martin

1 Strong transfinite ordinal dimension, 21 (1969), 591-596. 54D15 54F45

Landweber, Peter S.

1 Complex bordism of classifying spaces, 27 (1971), 175-179.

$18 \mathrm{G} 20$ 18H10 20J05 55B15 55B20 55F40 55H25 57D90; 20D15 20D20

Lappan, Peter A.

1 (with Piranian, George) Holomorphic functions with dense sets of Plessner points, 21 (1969), 555-556.

Larsen, Max D.

1 Harrison primes in a ring with few zero divisors, 22 (1969), 111-116.

13A15; $13 \mathrm{~F} 05$

2 (with Kelly, Patrick H.) Valuation rings with zero divisors, 30 (1971), 426-430.

Larson, Loren C.

$13 \mathrm{~A} 15$

1 Nonstandard theory of Zariski rings, 29 (1971), 23-29. $13 \mathrm{C} 15$ 13H10; 02H25 Larson, Richard Gustavus

1 The order of the antipode of a Hopf algebra, 21 (1969), 167-170.

$16 \mathrm{~A} 24$ Lasota, Andrzej

1 (with Bernfeld, Stephen R.) Quickly oscillating solutions of autonomous ordinary 
LaTorre, D. $\mathbf{R}$.

1 A note on quotient semirings, 24 (1970), 463-465. 16A78; 16A66

2 Modular congruences and the Brown-McCoy radical for semigroups, 29 (1971), 427-433.

20M10; 16A21

Lau, K. K.

1 (with Bagley, Robert W.) Semidirect products of topological groups with equal uniformities, 29 (1971), 179-182. 22A05 22D05

Laursen, Kjeld B.

1 Symmetry of generalized group algebras, 25 (1970), 318-322. $\quad$ 43A20 46H99

2 Maximal two-sided ideals in tensor products of Banach algebras, 25 (1970), 475-480.

Lavine, Richard B.

$46 \mathrm{H} 10$

1 Absolute continuity of Hamiltonian operators with repulsive potential, 22 (1969), 55-60.

47A55; 47B47

Layman, J. W.

1 Expansion of analytic functions in exponential polynomials, 22 (1969), 519-522.

Lazarov, Connor

30A 16; 30A82

1 (with Vasquez, Alphonse T.) The difference construction in $K$-theory, 21 (1969), 315-317.

22C05 55B15; 22D12 81A78

Leadbetter, M. R.

1 (with Weissner, Edward W.) On continuity and other analytic properties of stochastic process sample functions, 22 (1969), 291-294.

60G15 60G17

Leavitt, William G.

1 Strongly hereditary radicals, 21 (1969), 703-705.

17A99 17E05; 16A21

2 Radical and semisimple classes with specified properties, 24 (1970), 680-687; Erratum: 25 (1970), 922.

16A21 17A99

3 (with Bruning, L. M.) Minimal generating sets for free modules, 27 (1971), 441-445.

Lebow, Arnold

$16 \mathrm{~A} 64 ; 16 \mathrm{~A} 48$

1 (with Finkelstein, M.) A note on 'Nth roots of operators', 21 (1969), 250.

Ledden, P. J.

1 Nonstable homotopy groups of Thom complexes, 29 (1971), 404-410.

Lediaev, John P.

55E99 55G05 55G10 55G45 57F99

1 (with Johnson, E. W.) A new characterization of Dedekind domains, 28 (1971), 63-64.

13F05; 13A15 13E05 13H99

\section{Lee, Dong Hoon}

1 (with $\mathrm{Wu}, \mathrm{Ta}$-sun) On existence of compact open normal subgroups of 0 -dimensional groups, 26 (1970), 526-528.

$22 \mathrm{DO5}$

2 On the centralizer of a subgroup of a Lie group, 30 (1971), 195-198.

22E15 22E40

Lee, R. A.

1 (with Cusick, T. W.) Sums of sets of continued fractions, 30 (1971), 241-246.

Lee, Wuhan

$10 \mathrm{~F} 20$ 
1 On the compactness of the structure space of a ring, 21 (1969), 191-193.

Lee, Yu-lee

1 On a class of finer topologies with the same class of homeomorphisms, 21 (1969), 129-133.

Leech, Robert B.

1 On the characterization of $\mathscr{\&}(B)$ spaces, 23 (1969), 518-520. $\quad$ 46E40 47A45

Leela, $\mathbf{S}$.

1 (with Ladas, Gerasimos and Lakshmikantham, V.) On the perturbability of the asymptotic manifold of a perturbed system of differential equations, 27 (1971), 65-71.

34D05 34E10

Leighton, Walter

1 The conjugacy function, 24 (1970), 820-823. 34C10

2 (with Ke, William Oo Kian) A comparison theorem, 28 (1970), 185-188.

Leitzel, J. R. C.

$34 \mathrm{C} 10$

1 Galois cohomology and class number in constant extension of algebraic function fields, 22 (1969), 206-208.

$12 \mathrm{G} 05$

2 Class number in constant extensions of elliptic function fields, 25 (1970), 183-188.

Lemire, F. W.

$14 \mathrm{G} 15$

1 Weight spaces and irreducible representations of simple Lie algebras, 22 (1969), 192-197.

17B10 17B20

\section{Lenzing, Helmut}

1 A homological characterization of Steinitz rings, 29 (1971), 269-271.

16A10 16A50

Lercher, Bruce

1 (with Hindley, Roger) A short proof of Curry's normal form theorem, 24 (1970), 808-810.

$02 \mathrm{C} 20$

Letac, Gérard

1 A note about Wiener-Hopf sets, 22 (1969), 298-300.

60B15 60J15

Le Tourneau, J. J.

1 (with Comer, Stephen D.) Isomorphism types of infinite algebras, 21 (1969), 635639.

$08 \mathrm{~A} 15$

Leviatan, Dany

1 (with Lorch, Lee) A characterization of totally regular $[J, f(x)]$ transforms, 23 (1969), 315-319.

40C15 40D99

2 Remarks on some Tauberian theorems of Meyer-König, Tietz and Stieglitz , 29 (1971), 126-132.

40E05

\section{Levin, Mark}

1 (with Saxon, Stephen) Every countable-codimensional subspace of a barrelled space is barrelled, 29 (1971), 91-96.

46A07; 46A30

2 (with Saxon, Stephen) A note on the inheritance of properties of locally convex spaces by subspaces of countable codimension, 29 (1971), 97-102.

46A05 46A15

Levin, Simon A.

1 (with Block, Henry David) On the boundedness of an iterative procedure for solving a system of linear inequalities, 26 (1970), 229-235.

15A39 65F10; 65K05 93C40 94A30 
Lewis, Paul W.

1 Extension of operator valued set functions with finite semivariation, 22 (1969), 563-569.

2 (with Dinculeanu, Nicolae) Regularity of Baire measures, 26 (1970), 92-94.

\section{Libera, Richard J.}

1 (with Livingston, Arthur E.) On the univalence of some classes of regular functions, Lin, C.-S. 30 (1971), 327-336.

$30 \mathrm{~A} 32$

1 A note of the Kleinecke-Shirokov theorem and the Wintner-Wielandt-Halmos theorem, 27 (1970), 529-530. 47B05 47B05 47B47 47B47

Lin, You-feng

1 A problem of Bosák concerning the graphs of semigroups, 21 (1969), 343-346. $20 \mathrm{M} 99$

2 (with Ratti, Jogindar S.) Connectivity of the graphs of semirings: Lifting and product, 24 (1970), 411-414.

05C99 16A78

3 (with Ratti, Jogindar S.) The graphs of semirings. II, 30 (1971), 473-478.

Lindahl, R. J.

$05 \mathrm{C} 25 \quad 16 \mathrm{~A} 78$

1 A differentiation theorem for functions defined on the dyadic rationals, 30 (1971), 349-352.

26A24 26A48 42A56 42A62

Lindberg, John A., Jr.

1 A class of commutative Banach algebras with unique complete norm topology and continuous derivations, 29 (1971), 516-520.

46J05; 13B25

Lindner, Charles $\mathbf{C}$.

1 Extending partial permutation matrices, 24 (1970), 834.

2 Construction of quasigroups using the singular direct product, 29 (1971), 263-266.

05B30 20N05

Lipman, Joseph

1 On the Jacobian ideal of the module of differentials, 21 (1969), 422-426.

Lipschutz, Seymour

$13 \mathrm{H} 05 ; 14 \mathrm{~B} 05$ 14E 15

1 . On the conjugacy problem and Greendlinger's eighth-groups, 23 (1969), 101-106.

Lister, F. M.

20F10

1 Tame boundary sets of crumpled cubes in $E^{3}, 25$ (1970), 377-378.

Liu, Chen-tung

$57 \mathrm{~A} 10 ; 54 \mathrm{C} 25$

1 The a-closure $a X$ of a topological space $X, 22$ (1969), 620-624.

54D35; 54D25 54D60

2 An equivalent condition for the existence of a measurable cardinal, 23 (1969), 605-607.

Liu, Ming-chit

54A20 54A25; 54D60

1 On a problem of Erdös, 21 (1969), 706-710.

$10 \mathrm{~K} 15$

2 On functions of bounded boundary rotation, 29 (1971), 345-348. 30A32

Livingston, Albert E.

1 The coefficients of multivalent close-to-convex functions, 21 (1969), 545-552. 
Livingston, Arthur E.

30A32 30A34

1 (with Libera, Richard J.) On the univalence of some classes of regular functions, 30

(1971), 327-336.

$30 \mathrm{~A} 32$

Locke, Phil

1 On the asymptotic behavior of linear systems, 25 (1970), 93-95.

$34 \mathrm{D} 05$

Loeb, Peter Adolf

1 Compactifications of Hausdorff spaces, 22 (1969), 627-634. 54D35; 54D10 Lonergan, Francis D.

1 A characterization of cellular arcs in euclidean 3-space, 21 (1969), 153-154.

Long, Paul E.

57A 10

1 Concerning semiconnected maps, 21 (1969), 117-118.

54C10; 54D05

2 (with McGehee, Earl E., Jr.) Properties of almost continuous functions, 24 (1970), 175-180.

54C10; 54D05 54D45

Lorch, Lee

1 (with Leviatan, Dany) A characterization of totally regular $[J, f(x)]$ transforms, 23 (1969), 315-319.

40C15 40D99

Lorenz, Falko

1 A remark on real characters of compact groups, 21 (1969), 391-393.

Lorimer, P. J.

$20 \mathrm{C} 2022 \mathrm{C} 05$

1 A class of projective planes of cubic order, 21 (1969), 93-95.

17A30 50D35 Lovelady, David Lowell

1 A variation-of-parameters inequality, 26 (1970), 598-602.

34A40 34G05

2 Bounded solutions of Stieltjes integral equations, 28 (1971), 127-133.

45A05 45M10

Loveland, L. D.

1 Tameness implied by extending a homeomorphism to a point, 23 (1969), 287-293.

57A $10 ; 55 \mathrm{~A} 30$ 57A 50

2 A 2-sphere of vertical order 5 bounds a 3-cell, 26 (1970), 674-678.

55A30 55A40 57A50; 57A10 57A35

Loynes, R. M.

1 An invariance principle for reversed martingales, 25 (1970), 56-64.

60B10 60G45 60J65 62E20; 60B05 60F05 60G50 62E15

Luchins, Edith $\mathbf{H}$.

1 Completion of norms for $C(X, Q), 28$ (1971), 478-480.

$46 \mathrm{~J} 10 ; 46 \mathrm{~J} 99$

Ludescher, W. H.

1 (with Kenelly, John W., Jr.; Hare, William R. and Evans, B. D.) Convex components, extreme points, and the convex kernel, 21 (1969), 83-87. 52A30 Luh, Jiang

1 (with Koh, Kwangil) On a finite dimensional quasi-simple module, 25 (1970), 801-807.

16A64; 16A12 16A42

Luther, Norman Y.

1 Locally compact spaces of measures, 25 (1970), 541-547.

28A 10

Lutzer, David J.

1 A metrization theorem for linearly orderable spaces, 22 (1969), 557-558.

54E35 54F05; 06A45 
2 (with Bennett, Harold R.) Separability, the countable chain condition and the Lindelöf property in linearly orderable spaces, 23 (1969), 664-667.

54A25 54F05; 06A45 54D20

Macdonald, Ian D.

1 Solution of the Hughes problem for finite p-groups of class $2 p-2,27$ (1971), 39-42.

MacLachlan, C.

20D15 20D25

1 Modulus space is simply-connected, 29 (1971), 85-86.

30A46 30A58 40A58; 14K10 20H10 30A60 53C60

MacNerney, J. S.

1 Dense embeddings of Hilbert spaces, 24 (1970), 92-94. $46 \mathrm{C10}$ Madell, Robert L.

1 Chains which are coset spaces of tl-groups, 25 (1970), 755-759.

06A45 06A55 54F05; 06A45 22A25 22A99 54F05

Madison, Eugene W.

1 (with Lachlan, A. H.) Computable fields and arithmetically definable ordered fields, 24 (1970), 803-807.

$12 \mathrm{~J} 15$ 12L99

Magid, Andy $\mathbf{R}$.

1 Commutative algebras of Hochschild dimension one, 24 (1970), 530-532.

$13 \mathrm{~B} 0518 \mathrm{H} 20$

Magnus, Arne

1 The connection between P-fractions and associated fractions, 25 (1970), 676-679.

40A 15

Maharam, Dorothy

1 (with Kemperman, J. H. B.) $R^{c}$ is not almost Lindelöf, 24 (1970), 772-773.

Mahavier, William S.

1 Arcs in inverse limits on [0, 1] with only one bonding map, 21 (1969), 587-590.

Mahowald, Mark E.

26A48 54F20; 54F15

1 (with Gitler, Samuel and Milgram, R. James) Secondary cohomology operations and complex vector bundles, 22 (1969), 223-229.

55F25 55F40 55G10 55G20; 55G45 57F15

Mann, Avino'am

1 On subgroups of finite solvable groups, 22 (1969), 214-216. 20D10 Manougian, Manoug N.

1 On the convergence of a sequence of Perron integrals, 23 (1969), 320-322.

2 The Perron integral and existence and uniqueness theorems for a first order nonlinear differential equation, 25 (1970), 34-38. 34A10

\section{Mansfield, Richard}

1 The solution to one of Ulam's problems concerning analytic sets. II, 26 (1970), 539-540.

02K30 04A15 28A05 54H05

Marden, Morris

1 On composite abstract homogeneous polynomials, 22 (1969), 28-33.

15A24; 12D10

2 Logarithmic derivative of an entire function, 28 (1971), 513-518.

30A62 30A64; 30A08 
Markley, Nelson G.

1 On the number of recurrent orbit closures, 25 (1970), 413-416. 54H20 57A05 Marsden, Jerrold E.

1 (with Weinstein, Alan D.) A comparison theorem for Hamiltonian vector fields, 26 (1970), 629-631.

34C35

Martin, John C.

1 (with Yap, Leonard Y. H.) The algebra of functions with Fourier transforms in $L^{p}$, 24 (1970), 217-219.

43A15

Martin, Robert H., Jr.

1 A bound for solutions of Volterra-Stieltjes integral equations, 23 (1969), 506-512.

45D05

2 A global existence theorem for autonomous differential equations in a Banach space, 26 (1970), 307-314.

34A 10 34G05

Martindale, Wallace S., III

1 When are multiplicative mappings additive ?, 21 (1969), 695-698.

16A32; 16A 12

2 Primitive rings with involution whose symmetric elements satisfy a generalized polynomial identity, 24 (1970), 508-511. 16A20 16A28 16A42; 16A38

Marx, Morris L.

1 The Gauss realizability problem, 22 (1969), 610-613. 05C10; 55A15

2 (with Verhey, Roger F.) Interior and polynomial extensions of immersed circles, 24 $\begin{array}{ll}\text { (1970), 41-49. } & \text { 30A90 }\end{array}$

Marzec, R. P.

1 (with Franks, R. L.) $A$ theorem on mean-value iterations, 30 (1971), 324-326.

Mason, A. W.

26A18 47H10 54H25; 40A05 65Q05

1 On a theorem by Leon Greenberg, 23 (1969), 18-23.

Mason, J. H.

1 Geometrical realization of combinatorial geometries, 30 (1971), 15-21.

Mathews, J. H.

$05 \mathrm{~B} 2505 \mathrm{~B} 35$

1 Asymptotic behavior of light interior functions defined in the unit disk, 24 (1970), 79-81.

30A72

2 Asymptotic values of normal light interior functions defined in the unit disk, 24 (1970), 691-695.

30A72 30A90

Mattson, Don A.

1 Extensions of proximity functions, 26 (1970), 347-351.

54E05 54E10; 54C20 54C45

Mattuck, Arthur

1 On the symmetric product of a rational surface, 21 (1969), 683-688.

$14 \mathrm{C} 25 \quad 14 \mathrm{~J} 25$

2 Complete ideals and monoidal transforms, 26 (1970), 555-560.

13B20 14A05 14A25 14J15; $13 \mathrm{C} 99$

May, J. Peter

1 Some remarks on the structure of Hopf algebras, 23 (1969), 708-713.

May, Warren L. 
1 Unit groups of infinite abelian extensions, 25 (1970), 680-683.

Mayes, Vivienne

1 Some steady state properties of $\left(\int_{0}^{x} f(t) d t\right) / f(x), 22$ (1969), 672-677. 34D05 McCarthy, Paul J.

1 Principal elements of lattices of ideals, 30 (1971), 43-45.

$13 \mathrm{~A} 15 ; 13 \mathrm{~F} 05$

McCarty, G. S., Jr.

1 (with Geroch, Robert P. and Kronheimer, E. H.) No topologies characterize differentiability as continuity, 28 (1971), 273-274.

54C05 54C30; 26A27

McCharen, John D.

1 (with Anderson, R. D.) On extending homeomorphisms to Fréchet manifolds, 25 (1970), 283-289.

58B05

McCool, James

1 The power problem for groups with one defining relator, 28 (1971), 427-430.

McCoy, R. A.

$20 \mathrm{~F} 10$

1 Annulus conjecture and stability of homeomorphisms in infinite-dimensional normed linear spaces, 24 (1970), 272-277.

15A60 46B05

2 (with Fletcher, Peter and Slover, Rebecca Ellen) On boundedly metacompact and boundedly paracompact spaces, 25 (1970), 335-342. $\quad$ 54D15 54D20 54F45

3 (with Curtis, Douglas W.) Stable homeomorphisms on infinite-dimensional normed $\begin{array}{ll}\text { linear spaces, } 28 \text { (1971), 496-500. 58B05 } & \end{array}$

McCrimmon, Kevin

1 A note on finite division rings, 23 (1969), 598-600. 17A05

2 Koecher's principle for quadratic Jordan algebras, 28 (1971), 39-43. $\quad$ 17C05

McGehee, Earl E., Jr.

1 (with Long, Paul E.) Properties of almost continuous functions, 24 (1970), 175-180.

McIntosh, Alan G. R.

54C10; 54D05 54D45

1 Counterexample to a question on commutators, 29 (1971), 337-340. 47B47

McLaughlin, James R.

1 Rademacher series with nondifferentiable sums, 23 (1969), 140-143. $\quad 42$ A56

McLaughlin, Renate

1 An exceptional set for inner functions, 30 (1971), 545-546. $\quad 30 \mathrm{A76}$; 30A72

McMillan, D. R., Jr.

1 (with Row, Harry) Tangled embeddings of one-dimensional continua, 22 (1969), 378-385.

57A10 57A35

McMillan, J. E.

1 Cluster sets of meromorphic functions, 23 (1969), 148-150.

30A72

McPherson, James M.

1 A sufficient condition for an arc to be nearly polyhedral, 28 (1971), 229-233.

McShane, Edward James

55A30; 57A 10

1 (with Warfield, R. B., Jr.) Addenda and corrigenda to 'On Filippov's implicit functions lemma', 21 (1969), 496-498.

26A57

Meek, James L.

1 Subharmonic versions of Fatou's theorem, 30 (1971), 313-317. 31 A20 31C05 Megibben, Charles K.

1 Absolutely pure modules, 26 (1970), 561-566.

16A52 16A60; 18G05 
Menon, K. V.

1 An inequality of Schur and an inequality of Newton, 22 (1969), 441-449.

2 On the convolution of logarithmically concave sequences, 23 (1969), 439-441.

Merkes, E. P.

1 (with Wright, D. J.) On the univalence of a certain integral, 27 (1971), 97-100.

$40 A 99$

Merryman, Emory Hughes

1 An arcwise connected dense Hamel basis for Hilbert space, 26 (1970), 126-128.

Metcalf, Frederic T.

$46 \mathrm{~B} 15$

1 (with Bownds, John M.) An extension of the Nagumo uniqueness theorem, 27 (1971), 313-316.

$34 \mathrm{~A} 10$

Métivier, Michel

1 On strong measurability of Banach valued functions, 21 (1969), 747-748.

Metzger, Thomas A.

28A45

1 (with Rao, K. V. R.) On integrable and bounded automorphic forms, 28 (1971), 562-566.

30A58; 30A46

Meyer, Jean-Pierre

1 The Künneth formula and abelian monoids, 26 (1970), 699-700.

55B20 55D15 55D20 55E05 55H25 55H99 55J25; 55D45 55G45

Michael, Ernest A.

1 A theorem on perfect maps, 28 (1971), 633-634.

Mielke, M. V.

1 Cobordism properties of manifolds of small category, 21 (1969), 332-334.

Miles, Joseph

55C30 57D20 57D65 57D75

1 A note on Ahlfors' theory of covering surfaces, 21 (1969), 30-32.

$30 \mathrm{~A} 68$

Milgram, R. James

1 (with Gitler, Samuel and Mahowald, Mark E.) Secondary cohomology operations and complex vector bundles, 22 (1969), 223-229.

Miller, Donald J.

55F25 55F40 55G10 55G20; 55G45 57F15

1 The automorphism group of a product of graphs, 25 (1970), 24-28.

Miller, Gary Glenn

$05 \mathrm{C} 25 ; 20 \mathrm{~B} 25$

1 Countable connected spaces, 26 (1970), 355-360.

Miller, James

54A25 54D05 54G15; 54D10 54E25

1 Convex meromorphic mappings and related functions, 25 (1970), 220-228.

Miller, Richard K.

$30 \mathrm{~A} 32$

1 Admissibility and nonlinear Volterra integral equations, 25 (1970), 65-71.

Miller, Sanford S.

$45 \mathrm{D} 05$

1 The Hardy class of a Bazilevič function and its derivative, 30 (1971), 125-132. 
30A32 30A34 30A78

2 (with Keogh, F. R.) On the coefficients of Bazilevič functions, 30 (1971), 492-496.

30A32 30A34

Millman, R. S.

1 Geodesics in metrical connections, 30 (1971), 551-555.

53B05 53B20; 53C05 53C20

Mills, W. H.

1 The degrees of the factors of certain polynomials over finite fields, 25 (1970), 860-863.

$10 \mathrm{C05}$

Milnes, Paul

1 Identities of group algebras, 29 (1971), 421-422.

22D25; 22D05 22D35

Minassian, Donald P.

1 On the direct product of V-groups, 30 (1971), 434-436.

06A55; 06A60 Minc, Henryk

1 On lower bounds for permanents of $(0,1)$ matrices, 22 (1969), 117-123.

$15 \mathrm{~A} 1515 \mathrm{~A} 36$

Miranda, Guillermo

1 Regularization of singular systems of integral equations with kernels of finite doublenorm on $L_{\infty}, 26$ (1970), 423-427.

45E99 47G05; 45E05

Mitchell, George E.

1 The image of $\mathscr{U} *(X) \rightarrow N_{*}(X), 26(1970), 505-508 . \quad$ 55H99 57D75; 57D20 Miyadera, Isao

1 Note on nonlinear contraction semigroups, 21 (1969), 219-225.

47H99.

Monk, J. Donald

1 Provability with finitely many variables, 27 (1971), 353-358. $\quad$ 02B10 02J18

Montgomery, Susan

1 Polynomial identity algebras with involution, 27 (1971), 53-56.

$16 \mathrm{~A} 28$ 16A38; $16 \mathrm{~A} 48$

2 A generalization of a theorem of Jacobson, 28 (1971), 366-370.

16A28 16A68 16A70; 16A38

Moore, Berrien, III

1 The Szegö infimum, 29 (1971), 55-62.

47B35; 46C10

Moore, Theral $\mathbf{O}$.

1 (with Hadlock, E. H.) A new definition of a reduced form, 25 (1970), 105-113.

$10 \mathrm{C} 05$

Moran, Daniel A.

1 Approximating residual sets by strongly residual sets, 25 (1970), 752-754.

54C55 57A15; 54C10 54F40 57A10

Morash, Ronald P.

1 The orthomodular identity and metric completeness of the coordinatizing division ring, 27 (1971), 446-448; Erratum: 29 (1971), 627.

06A30; 81A12

Mordell, Louis Joel

1 On some sextic diophantine equations of genus 2,21 (1969), 347-350.

10B15 14G05

Mordeson, John N.

1 (with Vinograde, Bernard) Note on relative P-bases of purely inseparable extensions, 22 (1969), 587-590.

12F 15

Morris, Peter D. 
1 (with Lacey, H. Elton) On spaces of type $A(K)$ and their duals, 23 (1969), 151-157.

Morrow, James A.

1 The tangent bundle of the long line, 23 (1969), $458 . \quad 55 \mathrm{~F} 25$

Morse, Anthony P.

1 Subfunction structure, 21 (1969), 321-323.

46E05

Mosak, Richard D.

1 Central functions in group algebras, 29 (1971), 613-616.

\section{Mott, Joe Leonard}

22D05 22D15 43A20

1 (with Gilmer, Robert W., Jr.) An algebraic proof of a theorem of A. Robinson, 29 (1971), 461-466.

14A25; $13 \mathrm{LO} 05$

Mozzochi, C. J.

1 On a Riemann sum construction of Rudin, 22 (1969), 718.

Mrówka, Stanislaw

$52 \mathrm{~A} 45 ; 26 \mathrm{~A} 4250 \mathrm{~B} 30$

1 Some strengthenings of the Ulam nonmeasurability condition, 25 (1970), 704-711.

04A15

Muckenhoupt, Benjamin

1 Mean convergence of Jacobi series, 23 (1969), 306-310.

$33 \mathrm{~A} 65$ 42A52

2 Asymptotic forms for Laguerre polynomials, 24 (1970), 288-292.

Mukherjea, Arunava

33A65 34E05

1 (with Tserpes, Nicholas) Idempotent measures on locally compact semigroups, 29 (1971), 143-150.

Mukherjee, N. P.

1 The hyperquasicenter of a finite group. I, 26 (1970), 239-243.

20D25 Mumford, David

1 A remark on Mahler's compactness theorem, 28 (1971), 289-294.

22E40 32N15 32N99; 14H10 14H15 57E30

Murasugi, Kunio

1 The Arf invariant for knot types, 21 (1969), 69-72.

$55 \mathrm{~A} 25$

2 The commutator subgroups of the alternating knot groups, 28 (1971), 237-241.

55A05 55A25

Murdeshwar, M. G.

1 (with Deo, S. G.) On a system of integral inequalities, 26 (1970), 141-144.

Myers, Donald E.

$34 \mathrm{~A} 40$

1 An additive decomposition theorem for analytic functions, 27 (1971), 525-526.

Nadler, Sam B., Jr.

20D15 20D30

1 (with Ward, L. E., Jr.) Concerning continuous selections, 25 (1970), 369-374.

$54 \mathrm{C} 65$; 54F50

Nakamoto, Ritsuo

1 (with Furuta, Takayuki) Certain numerical radius contraction operators, 29 (1971), 521-524.

47A 10 47A30

Nandakumar, N. R.

1 A note on derivation pairs, 21 (1969), 535-539.

Narayana Rao, M. L.

30A98 46E25 
1 (with Zemmer, Joseph L., Jr.) A question of Foulser on $\lambda$-systems of characteristic two, 21 (1969), 373-378.

12K05; 50D35

2 (with Wilke, F. W.) A necessary condition that two finite quasi-fields coordinatize isomorphic translation planes, 24 (1970), 124-125.

$12 \mathrm{~K} 0550 \mathrm{D} 35$

3 A note on Moufang Veblen-Wedderburn systems, 24 (1970), 409-410.

$17 \mathrm{~A} 30$ 50D35; $12 \mathrm{~K} 05$

4 Characterization of Foulser's $\lambda$-systems, 24 (1970), 538-544.

Nassif, $\mathbf{M}$.

17A30 50D35; $12 \mathrm{~K} 05$

1 On the transpose of simple sets of polynomials effective in Faber regions, 25 (1970), 209-219.

Nath, B.

1 A general differential equation for classical polynomials, 27 (1971), 522-524.

Natzitz, Boaz

33A45 33A50 33A65 33A70

1 A note on interpolation, 25 (1970), 918. $46 \mathrm{~J} 10$

Neggers, Joseph

1 (with Chwe, Byoung-song) On the extension of linearly independent subsets of free modules to bases, 24 (1970), 466-470. 16A64; 16A08

Nehari, Zeev

1 (with Friedland, Schmuel) Univalence conditions and Sturm-Liouville eigenvalues, 24 (1970), 595-603.

34B25

\section{Nelson, Evelyn}

1 (with Burris, S.) Embedding the dual of $\Pi_{m}$ in the lattice of equational classes of commutative semigroups, 30 (1971), 37-39.

$08 \mathrm{~A} 15$ 20M05

Nelson, Stuart

$1 L^{2}$ asymptotes for the Klein-Gordon equation, 27 (1971), 110-116.

35B40 35L05; 81A33

$2 L^{2}$ asymptotes for Fourier transforms of surface-carried measures, 28 (1971), 134136.

41A60 42A72 42A92

Neuberger, J. W.

1 Analyticity and quasi-analyticity for one-parameter semigroups, 25 (1970), 488-494.

Neubrunn, Tibor

1 A note on quantum probability spaces, 25 (1970), 672-675. 28A05 Newman, Donald J.

1 (with Byrnes, J. S.) Completeness preserving multipliers, 21 (1969), 445-450.

$42 \mathrm{~A} 60$ 46E30

2 Translates are always dense on the half line, 21 (1969), 511-512.

$43 \mathrm{~A} 15$

3 On the number of binary digits in a multiple of three, 21 (1969), 719-721.

4 (with Byrnes, J. S.) A lower Jackson bound on $(-\infty, \infty), 26$ (1970), 71-72.

41 A 10 41A50

5 (with Kohn, S.) Multiplication from other operations, 27 (1971), 244-246.

12E99; $05 \mathrm{A05}$

6 (with Beller, E.) An $l_{1}$ extremal problem for polynomials, 29 (1971), 474-481. 
Newton, D.

1 On the entropy of certain classes of skew-product transformations, 21 (1969), 722726.

Newton, M. E.

1 The differential ideals $\left[y^{p}\right], 30$ (1971), 229-234. $12 \mathrm{H} 05$

Nichols, J. C.

1 Equivalent metrics giving different values to metric-dependent dimension functions, 23 (1969), 648-652.

54E35 54F45

Niederreiter, Harald

1 Orthogonal systems of polynomials in finite fields, 28 (1971), 415-422.

$12 \mathrm{C} 05 ; 12 \mathrm{C} 25$

Niven, Ivan

1 Averages of exponents in factoring integers, 22 (1969), 356-360. $\quad 10 \mathrm{H} 25$ Noble, Norman

1 A note on z-closed projections, 23 (1969), 73-76. 54B10 54C10; 54C30

Nobusawa, Nobuo

1 Crossed products of simple rings, 24 (1970), 18-21. 16A40 16A56; 16A42

Noll, Walter

1 Quasi-invertibility in a staircase diagram, 23 (1969), 1-4. 18A20 18E99 Noonan, James W.

1 Coefficients of functions with bounded boundary rotation, 29 (1971), 307-312.

30A32 30A34

Nordgren, Eric A.

1 Closed operators commuting with a weighted shift, 24 (1970), 424-428.

Nosal, Miloslav

1 Series convergence on Boolean algebras, 29 (1971), 211-212.

Novinger, W. $\mathbf{P}$.

1 (with Caveny, D. J.) Boundary zeros of functions with derivative in $H^{p}, 25$ (1970), 776-780.

$30 \mathrm{~A} 78$ 30A 80

Nunokawa, Mamoru

1 A note on convex and Bazilevič functions, 24 (1970), 332-335.

30A32

O'Brien, Thomas

1 Expansive homeomorphisms on compact manifolds, 24 (1970), 767-771.

54H20 57A15; 57A05 57E25

Ogg, A. P.

1 On product expansions of theta-functions, 21 (1969), 365-368. 30A16 33A25 O'Hara, P. J.

1 Divergence of interpolation polynomials in the complex domain, 25 (1970), 690-697.

Oksendal, B. $\mathbf{K}$.

$30 \mathrm{~A} 82$

1 (with Davie, Alexander M.) Rational approximation on the union of sets, 29 (1971), 581-584.

30A82 46J15

2 A short proof of the F. and M. Riesz Theorem, 30 (1971), 204. 28A10 30A06 Okuyama, Akihiro

1 A characterization of a space with countable infinity, 28 (1971), 595-597.

Olson, Milton Philip

54D40; 54D35 
1 The selfadjoint operators of a von Neumann algebra form a conditionally complete lattice, 28 (1971), 537-544.

46L10; 06A23 46A40

\section{O'Meara, Paul}

1 On paracompactness in function spaces with the compact-open topology, 29 (1971), 183-189.

54C35 54D20; 54E35

O'Neil, Patrick Eugene

1 A new criterion for uniform distribution, 24 (1970), 1-5.

$10 \mathrm{~F} 40$

2 Asymptotics in random (0, 1)-matrices, 25 (1970), 39-45.

05B20 05C35 15A15; 05C30 60C05

Onneweer, C. W.

1 On moduli of continuity and divergence of Fourier series on groups, 29 (1971), 109-112.

43A50 43A75

Ono, Tamio

1 A real analogue of the Gelfand-Neumark theorem, 25 (1970), 159-160. 46L05

Onose, Hiroshi

1 Oscillation theorems for nonlinear second order differential equations, 26 (1970), 461-464.

$34 \mathrm{C} 15$

Orey, Steven

1 (with Jamison, Benton) Subgroups of sequences and paths, 24 (1970), 739-744. 22A99 28A35 28A40 43A05 60F20 60G15 60G30; 60G17 62E10

Orlik-Pflugfelder, Hala

1 A special class of Moufang loops, 26 (1970), 583-586.

20N05; 50A99

Ornstein, Donald S.

1 A new proof of the paracompactness of metric spaces, 21 (1969), 341-342.

54D20 54E35

2 On a theorem of Orey, 22 (1969), 549-551.

26A86 28A65 47A65 60J10

Orsatti, Adalberto

1 (with De Marco, Giuseppe) Commutative rings in which every prime ideal is contained in a unique maximal ideal, 30 (1971), 459-466. 13A10 13A15; 54C40

Osofsky, B. L.

1 Loewy length of perfect rings, 28 (1971), 352-354.

16A46 16A48; 16A22 16A42

Otto, Albert D.

1 (with Davitt, Richard M.) On the automorphism group of a finite p-group with the central quotient metacyclic, 30 (1971), 467-472. 20D45

Ow, Wellington $\mathrm{H}$.

1 (with Goldstein, Myron) On the mean-value property of harmonic functions, 29 (1971), 341-344.

31 A05; 30A31

Owings, James C., Jr.

1 Commutativity and common fixed points in recursion theory, 24 (1970), 385-387.

Oxtoby, John C.

1 Homeomorphic measures in metric spaces, 24 (1970), 419-423.

Packel, Edward W.

26A42 28A10; 54E35

1 A semigroup analogue of Foguel's counterexample, 21 (1969), 240-244.

47B44 47D05

Padmanabhan, R.

1 (with Grätzer, György) On idempotent, commutative, and nonassociative groupoids, 
28 (1971), 75-80.

08A05 08A25; 20L05

Palais, Richard S.

1 When proper maps are closed, 24 (1969), 835-836.

$54 \mathrm{C} 10 ; 54 \mathrm{D} 50$

Palis, J.

1 S-explosions, 27 (1971), 85-90.

34C35

Park, Chul

1 A generalized Paley-Wiener-Zygmund integral and its applications, 23 (1969), 388400.

28A40 42A20 42A60

Parker, Donald B.

1 Wreath products and formations of groups, 24 (1970), 404-408. 20D10 20D40 Parnes, Milton N.

1 A distortion theorem for doubly connected regions, 26 (1970), 85-91. 30A30 Parthasarathy, T.

1 Product solutions for simple games. III, 23 (1969), 412-420. $90 \mathrm{D} 12$ Passman, D. S.

1 Central idempotents in group rings, 22 (1969), 555-556.

$20 \mathrm{C05}$

2 On the semisimplicity of twisted group algebras, 25 (1970), 161-166.

16A26 20C05

3 (with Coleman, Donald B.) Units in modular group rings, 25 (1970), 510-512.

$20 \mathrm{C} 05$

4 Idempotents in group rings, 28 (1971), 371-374. 20C05 Passow, E.

1 (with Raymon, L.) Polynomial approximation on $y=x^{a}, 24$ (1970), 781-783.

Patel, S. M.

$41 \mathrm{~A} 10$ 41A25

1 (with Ramanujan, P. B.) Operators whose ascent is 0 or 1, 29 (1971), 557-560.

Paterson, Alan L. T.

47B99; 47B05 47B47

1 Isometries between $B^{*}$-algebras, 22 (1969), 570-572.

46L05 47A10

Payne, Stanley E.

1 Generalized relative difference sets, 25 (1970), 46-50. Payne, T. H.

1 Effectively minimizing effective fixed-points, 30 (1971), 561-562.

$02 \mathrm{~F} 20$

Pazy, Amnon

1 Approximations of the identity operator by semigroups of linear operators, 30 (1971), 147-150.

47D05

Peek, Darwin E.

1 Baire functions and their restrictions to special sets, 30 (1971), 303-307.

26A15 26A21 41A30 54C30 54C50; 26A30 28A05 54A25 54B05 54B10

$54 \mathrm{C} 10$ 54E35 54E50

Pellicciaro, E. J.

1 A noncontractive fixed point theorem, 24 (1970), 627-628. $47 \mathrm{H} 10$

Perlis, Sam

1 Cyclicity of division algebras of prime degree, 21 (1969), 409-411.

Petersen, Bent E.

$16 \mathrm{~A} 40 ; 12 \mathrm{~F} 10$

1 The total symbol of a pseudo-differential operator, 30 (1971), 388-392.

$58 \mathrm{G} 15 ; 35 \mathrm{~S} 05$ 
Petersen, K. E.

1 Disjointness and weak mixing of minimal sets, 24 (1970), 278-280.

Peterson, Allan C.

$54 \mathrm{H} 15 ; 54 \mathrm{H} 20$

1 A theorem of Aliev, 23 (1969), 364-366. 34C10

Peterson, H. LeRoy

1 Irregular invariant measures related to Haar measure, 24 (1970), 356-361.

22A10 28A70

Petrich, Mario

1 On a class of completely semisimple inverse semigroups, 24 (1970), 671-676.

Pettis, B. J.

1 Cluster sets of nets, 22 (1969), 386-391. 54A20

Petty, C. M.

1 Equilateral sets in Minkowski spaces, 29 (1971), 369-374.

$50 \mathrm{C} 25$ 52A20 52A50; 52A25

Peyser, Gideon

1 On hyperbolic polynomials with multiple roots, 21 (1969), 667-670.

Pfaffenberger, William

$12 \mathrm{D} 1035 \mathrm{~L} 25$

1 On the ideals of strictly singular and inessential operators, 25 (1970), 603-607.

Pfaltz, John L.

47D99; 47B30

1 Semihomomorphisms of semimodular lattices, 22 (1969), 418-425. $\quad$ 06A30

Pfeffer, W.

1 (with Wilbur, W. John) A note on cluster points of a semihereditary stable system of sets, 21 (1969), 121-125.

54A20; 28A15

Phillips, R. G.

1 On the structure of nonstandard models of arithmetic, 27 (1971), 359-363.

Piech, M. Ann

$02 \mathrm{H} 20$

1 Regularity properties for families of measures on a metric space, 24 (1970), 307-311.

Pigno, Louis

28A 10; 54E35

1 Restrictions of $L^{p}$ transforms, 29 (1971), 511-515.

42A18 43A15

Pincus, Joel David

1 (with Rovnyak, James) A representation theorem for determining functions, 22 (1969), 498-502.

47A65 47B47

Pinsky, Mark A.

1 An elementary derivation of Khintchine's estimate for large deviations, 22 (1969), 288-290.

$60 \mathrm{~F} 10 ; 60 \mathrm{G} 50$

Piranian, George

1 (with Lappan, Peter A.) Holomorphic functions with dense sets of Plessner points, 21 (1969), 555-556.

30A72

Pittman, C. R.

1 An elementary proof of the triod theorem, 25 (1970), 919.

Plafker, Stephen M.

57A05; 54A25 54F20 
1 On decomposable operators, 24 (1970), 215-216.

Platek, Richard A.

$1 A$ note on the failure of the relativized enumeration theorem in recursive function theory, 25 (1970), 915-916.

2 A note on the cardinality of the Medvedev lattice, 25 (1970), $917 . \quad 02$ F30 Plemmons, Robert J.

1 Graphs associated with a group, 25 (1970), 273-275. $\quad 05 \mathrm{C} 20$ 05C25; 20B25 Poguntke, Detlev

1 Epimorphisms of compact groups are onto, 26 (1970), 503-504. 22C05 Pollard, Harry

1 (with Saari, Donald G.) An elementary Tauberian theorem of nonlinear type, 24 (1970), 593-594.

40E05

2 (with Darst, Richard B.) An inequality for the Riemann-Stieltjes integral, 25 (1970), 912-913.

26A42 26A84 26A86

Porter, Jack R.

1 (with Himmelberg, Charles J. and Van Vleck, F. S.) Fixed point theorems for condensing multifunctions, 23 (1969), 635-641.

$47 \mathrm{H} 10$

2 Not all semiregular Urysohn-closed spaces are Katětov-Urysohn, 25 (1970), 518-520.

Pothoven, Kenneth

54D10 54D25

1 Projective and injective objects in the category of Banach spaces, 22 (1969), 437-438.

\section{Poulsen, Ebbe Thue}

1 The minimax principle and uniqueness of the Friedrichs extension, 21 (1969), 508509.

Powell, Robert E.

1 (with Cox, Raymond H.) Regularity of net summability transforms on certain linear topological spaces, 21 (1969), 471-476. 40J05

Preston, C. J.

1 On the convergence of multiplicatively orthogonal series, 28 (1971), 453-455.

42A60; 40A05 42A20

Price, Thomas

1 (with Cantrell, James C. and Rushing, T. B.) A class of embeddings of $S^{n-1}$ and $B^{n}$ in $R^{n}, 29$ (1971), 208-210.

57A15 57A45

Priddy, Stewart B.

1 On the homotopy theory of simplicial Lie algebras, 25 (1970), 513-517.

55E05 55E25 55E40 55G10 55H15

Priestley, W. M.

1 A sequentially closed countable dense subset of $I^{I}, 24$ (1970), 270-271.

54B10;10E10 54A20 54A25

Proctor, Thomas Gilmer

1 Characteristic multipliers for some periodic differential equations, 22 (1969), 503508.

2 Periodic solutions for perturbed nonlinear differential equations, 24 (1970), 815-819.

Prosser, Reese T.

$34 \mathrm{C} 25$

1 Note on metric dimension, 25 (1970), 763-765. 
$2 A$ brief derivation of the Heisenberg commutation relations, 26 (1970), 640-641.

$81 \mathrm{~A} 20$

3 A comparison theorem for operators with compact resolvent, 27 (1971), 519-521.

Pugh, W. J.

47A10 47A55 47B05

1 Sums of functions of bounded index, 22 (1969), 319-323.

$30 A 64$

Putcha, Mohan S.

1 On Lie rings satisfying the fourth Engel condition, 28 (1971), 355-357.

Putnam, Calvin R.

17B05 17B30 17B40; 20F40 20F45

1 The spectra of subnormal operators, 28 (1971), 473-477.

47A10 47B20

Putz, H.

1 Transverse field implies normal microbundle, 23 (1969), 232-236.

Pym, John S.

$57 C 3557 C 50$

1 Idempotent probability measures on compact semitopological semigroups, 21 (1969), 499-501.

43A10; $22 \mathrm{~A} 10$

Quillen, Daniel G.

1 On the endomorphism ring of a simple module over an enveloping algebra, 21 (1969), 171-172.

16A64; 17B30 17B35

Radford, David E.

1 A free rank 4 Hopf algebra with antipode of order 4, 30 (1971), 55-58.

Radjavi, Heydar

1 Every operator is the sum of two irreducible ones, 21 (1969), 251-252. $\quad 47$ A15

2 (with Rosenthal, Peter) The set of irreducible operators is dense, 21 (1969), 256.

$47 \mathrm{~A} 15$

3 Products of Hermitian matrices and symmetries, 21 (1969), 369-372; Erratum: 26 (1970), 701.

15A57

4 (with Harrison, K. J. and Rosenthal, Peter) A transitive medial subspace lattice, 28 (1971), 119-121.

47A 15; 47C05

Rahman, Qazi Ibadur

1 (with Goodman, Adolph W. and Ratti, Jogindar S.) On the zeros of a polynomial and its derivative, 21 (1969), 273-274.

30A08

Rainwater, John

1 Local uniform convexity of Day's norm on $c_{0}(\Gamma), 22$ (1969), 335-339.

46B10 46E15

2 Regular matrices with nowhere dense support, 29 (1971), 361. 40C05 40D20

Ramanujan, P. B.

1 (with Patel, S. M.) Operators whose ascent is 0 or 1,29 (1971), 557-560.

47B99; 47B05 47B47

Ramirez, Donald E.

1 (with Dunkl, Charles F.) Multipliers on compact groups, 28 (1971), 456-460.

Ramras, Mark

$43 \mathrm{~A} 22 ; 46 \mathrm{H} 25$

1 On the vanishing of Ext, 27 (1971), 457-462.

Randall, Charles $\mathbf{H}$.

$13 \mathrm{C} 10$ 13D05; $13 \mathrm{H} 10$

1 A complete and countable orthomodular lattice is atomic, 21 (1969), 253. 
Randolph, J. W.

06А23 06A25 06А30

1 Finite groups with solvable maximal subgroups, 23 (1969), 490-492.

Rao, K. V. R.

20D10 20D25

1 (with Metzger, Thomas A.) On integrable and bounded automorphic forms, 28 (1971), 562-566.

\section{Ratti, Jogindar S.}

1 On a relation between absolute Abel and absolute Riesz summability, 21 (1969), 57-62.

40D25 40G10; 40C10

2 (with Goodman, Adolph W. and Rahman, Qazi Ibadur) On the zeros of a polynomial and its derivative, 21 (1969), 273-274.

30A08

3 Correction to "On strong Riesz summability factors of infinite series", 22 (1969), 723.

$40 \mathrm{~F} 05$

4 (with Lin, You-feng) Connectivity of the graphs of semirings: Lifting and product, 24 (1970), 411-414.

$05 \mathrm{C} 99$ 16A78

5 (with Lin, You-feng) The graphs of semirings. II, 30 (1971), 473-478.

Ravisankar, T. S.

$05 \mathrm{C} 25 \quad 16 \mathrm{~A} 78$

1 A note on a theorem of Kokoris, 21 (1969), 355-356.

17A15 17A25

2 A note on a theorem of Jacobson, 21 (1969), 753-754.

17A99 17B05 17B20 17B40 17E05

Raymon, L.

1 (with Passow, E.) Polynomial approximation on $y=x^{a}, 24$ (1970), 781-783.

Reade, Maxwell 0.

41A10 41A25

1 (with Zlotkiewicz, Eligiusz) On univalent functions with two preassigned values, 30 (1971), 539-544.

30A32

Reddy, William L.

1 (with Coven, Ethan M.) Limit set equivalences of replete semigroups, 23 (1969), 625-630.

Reeder, John

1 On the invertibility of general Wiener-Hopf operators, 27 (1971), 72-76. $47 \mathrm{~B} 35$

Rees, Elmer

1 An example on embedding up to homotopy type, 26 (1970), 217-218.

55D99 57C05 57C35; 55D10 57F99

Reid, James L.

1 An exact solution of the nonlinear differential equation $\ddot{y}+p(t) y$

$=q_{m}(t) / y^{2 m-1}, 27$ (1971), 61-62.

34A05 46L05

Reilly, Norman $\mathbf{R}$.

1 The number of permutational products of two finite groups, 25 (1970), 507-509.

Reilly, Robert C.

20D40

1 Applications of stereographic projections to submanifolds in $E^{m}$ and $S^{m}, 25$ (1970), 119-123.

53C40; 53A 10

Reiner, Irving

1 (with Lam, Tsit-yuen) Finite generation of Grothendieck rings relative to cyclic subgroups, 23 (1969), 481-489.

20C05 
Reis, Clive M.

1 (with Viswanathan, T. M.) A compactness property for prime ideals in Noetherian rings, 25 (1970), 353-356.

13A15; $13 \mathrm{C} 99$

Reneke, James A.

1 A product integral solution of a Stieltjes-Volterra integral equation, 24 (1970), 621-626.

Resnikoff, H. L.

1 Differential equations for automorphic forms in several complex variables, 24 (1970), 492-496.

$17 \mathrm{C} 3532 \mathrm{~N} 15$

Restrepo, Guillermo

1 An infinite dimensional version of a theorem of Bernstein, 23 (1971), 193-198.

Rhoads, Donald

$41 \mathrm{~A} 65 ; 46 \mathrm{~J} 10$

1 Quasi-compact operators in topological linear spaces, 25 (1970), 261-265.

Ribes, Luis

1 On a cohomology theory for pairs of groups, 21 (1969), 230-234.

Rice, Bart Francis

$18 \mathrm{H} 10 ; 20 \mathrm{~J} 99$

1 Quarternions and binary quadratic forms, 27 (1971), 1-7.

$10 \mathrm{C05}$

Rich, Michael

1 On nearly commutative nodal algebras in characteristic zero, 24 (1970), 563-565.

17A25 17A30 17E05

2 Some radical properties of s-rings, 30 (1971), 40-42. 17A99 17E05; 16A21 Richards, James W.

1 Abelian f.p.f. operator groups of type ( $p, p), 29$ (1971), 1-9.

20B25 20D10 20D45; 20K05

Richardson, G. D.

1 A class of uniform convergence structures, 25 (1970), 399-402.

54A20 54D10 54E15; 54D30

2 A Stone-Čech compactification for limit spaces, 25 (1970), 403-404.

54A20 54D35; 54A05

3 Completions of uniform convergence spaces, 29 (1971), 159-164. 54A20 54E15 Richen, Forrest

1 Decomposition numbers of p-solvable groups, 25 (1970), 100-104.

20D20 20D30

Richman, Fred

1 (with Walker, Elbert A.) Extending Ulm's theorem without group theory, 21 (1969), 194-196.

04A20 20K25

Riddell, R. C.

1 A note on Palais' axioms for section functors, 25 (1970), 808-810.

55F25 58A99 58D15; 58B10

2 (with Insley, R. B.) Commuting operator solutions of algebraic equations, 28 (1971), 461-463.

47A50

Rider, D. G.

1 Functions which operate in the Fourier algebra of a compact group, 28 (1971), 525-530.

43A20 43A30; 46J99 


\section{Rigelhof, Roger}

1 Subalgebras of group algebras, 23 (1969), 404-408.

2 Invariant measures on locally compact semigroups, 28 (1971), 173-176. 20M10 22B05 28A70

Riley, John A.

1 The maximal ideals in quaternion orders, 28 (1971), 436-438.

16A18 16A66; 13H99

\section{Rinehart, George S.}

1 Note on the homology of a fiber product of groups, 24 (1970), 548-552.

Roach, F. A.

$18 \mathrm{H} 1020 \mathrm{~J} 05$

1 Continued fractions over an inner product space, 24 (1970), 576-582. $\quad 40 \mathrm{C05}$

2 The parabola theorem for continued fractions over a vector space, 28 (1971), 137-146.

$40 \mathrm{~A} 15$

Roberson, Fred A.

1 (with Gray, William J.) On the near equicontinuity of transformation groups, 23 (1969), 59-63.

$34 \mathrm{~A} 10$ 54H15; 54H20 54H25

2 A theorem on near equicontinuity of transformation groups., 27 (1971), 189-191.

Roberts, Leslie G.

54H15 54H20; 54D45 54E35

$1 K_{1}$ of projective r-space, 26 (1970), 587-592.

$14 \mathrm{~F} 15$

Robertson, Jack $M$.

1 Retracting diffeomorphisms of n-spheres, 24 (1970), 57-59.

Robertson, M. S.

57D50 57E05 57E25 58D99

1 A distortion theorem for analytic functions, 28 (1971), 551-556.

\section{Robinson, Julia}

30A42 30A76; 30A36

1 Finitely generated classes of sets of natural numbers, 21 (1969), 608-614.

2 Unsolvable diophantine problems, 22 (1969), 534-538.

$02 \mathrm{~F} 25$

Robson, J. C.

1 (with Griffith, Phillip A.) A theorem of Asano and Michler, 24 (1970), 837-838.

Rogers, J. W., Jr.

$16 \mathrm{~A} 12$ 16A46

1 Continua not an inverse limit with a single bonding map on a polyhedron, 21 (1969), 281-283.

2 On mapping indecomposable continua onto certain chainable indecomposable continua, 25 (1970), 449-456.

54C10 54F20; 54B25

Rogers, James Ted, Jr.

1 (with Tollefson, Jefferey L.) Homeomorphism groups of weak solenoidal spaces, 28 (1971), 242-246.

54B25 54H15; 57A 15

2 Embedding the hyperspaces of circle-like plane continua, 29 (1971), 165-168.

54F20; 54B20 54B25 57A05 57A10 57D12

3 (with Tollefson, Jefferey L.) Homogeneous inverse limit spaces with nonregular covering maps as bonding maps, 29 (1971), 417-420. $\quad$ 54B25 57A15; 54G20

Rosen, Ronald H. 
Rosencrans, S. I.

1 An extremal property of stochastic integrals. , 28 (1971), 223-228.

\section{Rosenfeld, $\mathbf{M}$.}

$60 \mathrm{H} 0560 \mathrm{~J} 65 ; 35 \mathrm{~K} 1535 \mathrm{~L} 15$

1 Graphs with a large capacity, 26 (1970), 57-59.

05C99; 94A20

\section{Rosenthal, Aaron}

1 Riemannian manifolds of constant k-nullity, 22 (1969), 473-475.

\section{Rosenthal, Paul}

1 On the zeros of the Bergman function in doubly-connected domains, 21 (1969), 33-35.

30A30 30A31

\section{Rosenthal, Peter}

1 (with Radjavi, Heydar) The set of irreducible operators is dense, 21 (1969), 256.

$47 \mathrm{~A} 15$

2 Weakly closed maximal triangular algebras are hyperreducible, 24 (1970), 220.

3 (with Harrison, K. J. and Radjavi, Heydar) A transitive medial subspace lattice, 28 (1971), 119-121.

47A15; 47C05

Roth, B.

1 (with Kascic, Michael J., Jr.) A closed subspace of $\mathscr{Z}(\Omega)$ which is not an LF-space, 24 (1970), 801-802.

35E99 46A15

Roth, Emile Boyd

1 Quasi-reflexitivity and dual norms, 23 (1969), 164-166. 46B10

Rothman, Neal J.

1 (with Baker, John Warren) Separating points by semicharacters in topological semigroups, 21 (1969), 235-239.

22A15 22A20

Rothschild, B. L.

1 (with Kleitman, Daniel J.) The number of finite topologies, 25 (1970), 276-282.

\section{Rothschild, Linda Preiss}

05B30; 54A05

1 On uniqueness of quasi-split real semisimple Lie algebras, 24 (1970), 6-8.

\section{Rousseau, George}

$17 \mathrm{~B} 20$

1 (with Davies, Roy O. and Hayes, Allan) Complete lattices and the generalized Cantor theorem, 27 (1971), 253-258. 06 A 10; 06A20

Rovnyak, James

1 (with Pincus, Joel David) A representation theorem for determining functions, 22 (1969), 498-502.

47A65 47B47

\section{Row, Harry}

1 (with McMillan, D. R., Jr.) Tangled embeddings of one-dimensional continua, 22 (1969), 378-385.

57A10 57A35

Rubin, Leonard $\mathbf{R}$.

1 Recognizing certain factors of $E^{4}, 26$ (1970), 199-200. 57A10 57A15; 54B15 Rubinstein, Żalman

1 (with Saff, E. B.) Bounded approximation by polynomials whose zeros lie on a circle, 29 (1971), 482-486.

30A82 41A10; 30A06

Ruh, Ernst Alfred

1 Minimal immersions of 2 -spheres in $S^{4}, 28$ (1971), 219-222. 
Rupp, Russell D., Jr.

$53 \mathrm{~A} 10$ 53C40; 53B20 53C20

1 Uniqueness for second order linear parabolic operators, 22 (1969), 285-288.

Rushing, T. B.

$35 \mathrm{~K} 20 ; 35 \mathrm{~A} 05$

1 Realizing homeomorphisms by ambient isotopies, 23 (1969), 723-724.

$54 \mathrm{C} 05$ 55D99

2 Adjustment of topological concordances and extensions of homeomorphisms over pinched collars, 26 (1970), 174-177. 57A15 57A35 57A45 57A70; 57C99

3 (with Cantrell, James C. and Price, Thomas) A class of embeddings of $S^{n-1}$ and $B^{n}$ in $R^{n}, 29$ (1971), 208-210.

57A15 57A45

Russo, Bernard

1 Isometries of the trace class, 23 (1969), 213.

47D15; 46L10

Ryder, Gerald $\mathbf{H}$.

1 (with Wend, David V. V.) Oscillation of solutions of certain ordinary differential equations of nth order, 25 (1970), 463-469. $34 \mathrm{C} 10$

Saari, Donald G.

1 Some large $O$ nonlinear Tauberian theorems, 21 (1969), 459-462. 40E05

2 (with Pollard, Harry) An elementary Tauberian theorem of nonlinear type, 24 (1970), 593-594.

$40 \mathrm{E} 05$

Sabbagh, Gabriel

1 Endomorphisms of finitely presented modules, 30 (1971), 75-78. $13 \mathrm{C} 10$ 13E99 Saeki, Sadahiro

1 A characterization of $S H$-sets, 30 (1971), 497-503. 43A45; 43A20 43A25

Saff, E. B.

1 (with Twomey, J. B.) A note on the location of critical points of polynomials, 27 (1971), 303-308.

$30 \mathrm{~A} 08$

2 Regions of meromorphy determined by the degree of best rational approximation, 29 (1971), 30-38.

30A82 41A20

3 (with Rubinstein, Zalman) Bounded approximation by polynomials whose zeros lie on a circle, 29 (1971), 482-486.

30A82 41A10; 30A06

Saks, Victor

1 (with Stephenson, Robert M., Jr.) Products of M-compact spaces, 28 (1971), 279288.

54B10 54D30; 54A25 54D35

Salehi, Habib

1 (with Taylor, Gerald D.) Positive matrix $H^{1 / 2}$ and Hermitian matrix $H^{1}$ functions are constant, 26 (1970), 469-470.

30A78; $15 \mathrm{~A} 48$

2 On determination of the optimal factor of a nonnegative matrix-valued function, 29 (1971), 383-389.

15A15 15A24 15A42 15A48 15A54 30A96 39A25 60G25; 15A60 47A50

Samelson, Hans

1 Orientability of hypersurfaces in $R^{n}, 22$ (1969), 301-302.

55C99 57-01 57D40 57D99

Sandomierski, Francis L.

1 On semiperfect and perfect rings, 21 (1969), 205-207.

16A50

2 A note on the global dimension of subrings, 23 (1969), 478-480.

16A32 16A60; 16A50 
3 Some examples of right self-injective rings which are not left self-injective, 26 (1970), 244-245.

16A36 16A52; 16A64

Santos, Eugene S.

1 Probabilistic Turing machines and computability, 22 (1969), 704-710.

\section{Sarason, Donald}

02F10 94A35

1 (with Douglas, Ronald George) Fredholm Toeplitz operators, 26 (1970), 117-120.

\section{Satô, Shizuka}

1 On rings with a higher derivation, 30 (1971), 63-68.

47B30 47B35

Saworotnow, Parfeny P.

1 (with Friedell, J. C.) Trace-class for an arbitrary $H^{*}$-algebra, 26 (1970), 95-100.

46K $1547 \mathrm{~B} 10$

2 Trace-class and centralizers of an $H^{*}$-algebra, 26 (1970), 101-104.

\section{Saxon, Stephen}

$46 \mathrm{~K} 1547 \mathrm{~B} 10$

1 (with Levin, Mark) Every countable-codimensional subspace of a barrelled space is barrelled, 29 (1971), 91-96.

46A07; 46A30

2 (with Levin, Mark) A note on the inheritance of properties of locally convex spaces by subspaces of countable codimension, 29 (1971), 97-102.

46A05 46A15 Schacher, Murray M.

1 Cyclotomic splitting fields, 25 (1970), 630-633.

12B10 16A40 16A46

Schaefer, Helmut $\mathbf{H}$.

1 On the characteristic roots of real matrices, 28 (1971), 91-92. 15A18; 15A48 Schaeffer, David G.

1 A note on the representation of a solution of an elliptic differential equation near an isolated singularity, 23 (1969), 450-454.

$35 \mathrm{C} 9935 \mathrm{~J} 30$

2 An extension of Hartogs' theorem for domains whose boundary is not smooth, 25 (1970), 714-715.

32D10

\section{Scheinberg, Stephen}

1 A convergence question in $H^{p}, 30$ (1971), 120-124.

30A78; 60G25

Schelp, R. H.

1 (with Gudder, Stanley P.) Coordinatization of orthocomplemented and orthomodular posets, 25 (1970), 229-237. $\quad$ 06A10 20M15; 06A25 06A30 Schenkman, Eugene

1 The general product of two finitely generated abelian groups, 21 (1969), 202-204.

20D40

2 Some criteria for nilpotency in groups and Lie algebras, 21 (1969), 714-718.

17B05 17B40 20F35 20F40 20F55

3 The tower theorem for finite groups, 22 (1969), 458-459.

20D45; 20D35

Schmeidler, David

1 Fatou's lemma in several dimensions, 24 (1970), 300-306.

28A25 28A45

Schmidt, H. J., Jr.

1 On normal complements of $\mathscr{F}$-covering subgroups, 25 (1970), 457-459.

Schmidt, Wolfgang M.

20D10

1 Irregularities of distribution. V, 25 (1970), 608-614.

$52 \mathrm{~A} 45$

Schmitt, Klaus 
1 Periodic solutions of linear second order differential equations with deviating argument, 26 (1970), 282-285.

$34 \mathrm{C} 25$ 34K 15

Schneeberger, $\mathbf{C}$.

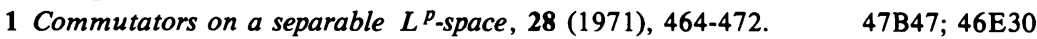
Schneider, Leo J.

1 Oscillation properties of the 2-2 disconjugate fourth order selfadjoint differential equation, 28 (1971), 545-550. 34C10

Schneider, W. J.

1 (with Barth, K. F.) An asymptotic analog of the F. and M. Riesz radial uniqueness theorem, 22 (1969), 53-54.

30A76; 30A88

2 (with Barth, K. F.) A short proof of a lemma of G. R. MacLane, 22 (1969),

Schober, G. E.

30A72

1 (with Hengartner, Walter) Analytic functions close to mappings convex in one direction, 28 (1971), 519-524. 30A32 30A34

Schochetman, Irwin

1 Dimensionality and the duals of certain locally compact groups, 26 (1970), 514-520.

22D30; 43A60

2 Nets of subgroups and amenability, 29 (1971), 397-403.

Schrader, Keith W.

22D05 22D40 22D99; 22D10 22D30

1 (with Waltman, Paul E.) An existence theorem for nonlinear boundary value problems, 21 (1969), 653-656.

34B15

Schreiber, Michel

1 (with Targonski, György I.) Carleman and semi-Carleman operators, 24 (1970), 293-299.

Schubert, C. F.

1 Invariant subspaces with invariant complements, 22 (1969), 85-90.

Schultz, Reinhard E.

30A78 46E20

1 The nonexistence of free $S^{1}$ actions on some homotopy spheres, 27 (1971), 595-597.

57D60 57E15 57E25

Schumaker, Larry L.

1 (with Jerome, Joseph W.) Applications of $\epsilon$-entropy to the computation of n-widths, 22 (1969), 719-722.

41A45 46B99

Schumitzky, Alan

1 (with Kagiwada, Harriet $\mathrm{H}$. and Kalaba, Robert E.) A representation for the solution of Fredholm integral equations, 23 (1969), 37-40. 45B05

Schupp, Paul E.

1 On the substitution problem for free groups, 23 (1969), 421-423. $20 \mathrm{~F} 10$

Schwabauer, Robert

1 Commutative semigroup laws, 22 (1969), 591-595.

20M99; 20E10

Schwabhäuser, Wolfram

1 The connection between two geometrical axioms of $H$. N. Gupta, 22 (1969), 233-234.

Schwartz, Alan L.

50A05 50A20; 12D15

1 An inversion theorem for Hankel transforms, 22 (1969), 713-717.

$44 \mathrm{~A} 15$

2 On the ideal structure of the algebra of radial functions, 26 (1970), 621-624. 
Schwartz, Benjamin L.

1 (with Beineke, Lowell W.) Locally finite self-interchange graphs, 27 (1971), 8-12.

05C99

Schwartzbauer, T.

1 (with Akcoglu, M. A. and Chacon, Rafael V.) Commuting transformations and mixing, 24 (1970), 637-642.

2 A general method for approximating measure preserving transformations, 24 (1970), 643-648.

$28 \mathrm{~A} 65$

\section{Scott, J. B.}

1 (with Etgen, Garret J.) On the conjugate points of fourth order, selfadjoint linear differential equations, 29 (1971), 349-350.

$34 \mathrm{C} 10$

Scott, W. R.

1 Semi-isomorphisms of certain infinite permutation groups, 21 (1969), 711-713.

$20 \mathrm{E} 99$

2 (with Amberg, Bernhard) Products of Abelian subgroups, 26 (1970), 541-547.

20F25

\section{Seebeck, Charles L. III}

1 Trme arcs on wild cells, 29 (1971), 197-201.

57A15 57A35; 57D40 Seeley, Robert T.

1 Eigenfunction expansions of analytic functions, 21 (1969), 734-738.

32L99 35P10; $42 \mathrm{~A} 60$

\section{Segal, David M.}

1 On the stable homotopy of quaternionic and complex projective spaces, 25 (1970), 838-841.

55E10 55H15 57F15 57F20

2 Divisibility conditions on characteristic numbers of stably symplectic manifolds, 27 (1971), 411-415.

57D20 57D90; 55H15

Sehgal, V. M.

1 A fixed point theorem for mappings with a contractive iterate, 23 (1969), 631-634.

54C10 54E40 54E50 54H25

2 (with George, John H. and Smithson, Raymond E.) Application of Liapunov's direct method to fixed point theorems, 28 (1971), 613-620.

$54 \mathrm{C} 60$ 54H25; 54E35 54E45

3 (with Thomas, James W.) A fixed point theorem for semigroups of mappings with a contractive iterate, 29 (1971), 565-568.

47D05 47H10

Seidenberg, A.

1 Abstract differential algebra and the analytic case. II, 23 (1969), 689-691.

$12 \mathrm{H} 05$

2 On the length of a Hilbert ascending chain, 29 (1971), 443-450.

Sen, Rabindra Nath

1 Correction to a theorem of mine, 27 (1971), 341-342. 53B20

Sentilles, F. Dennis

1 Existence of regular finite invariant measures for Markov processes, 21 (1969), 318-320.

$28 \mathrm{~A} 65$ 60J 25

Severo, Norman C.

1 (with Slivka, John) On the strong law of large numbers, 24 (1970), 729-734.

60F10 60F15 62E20; 60E05 60F05 60F20 


\section{Shafaat, Ahmad}

1 Subcartesian products of finitely many finite algebras, 26 (1970), 401-404.

Shaffer, Dorothy Browne

1 On the convexity of lemniscates, 26 (1970), 619-620. 30A06 Shannon, Richard T.

1 The rank of a flat module, 24 (1970), 452-456.

Shapiro, J. H.

1 (with Gregory, D. A.) Nonconvex linear topologies with the Hahn Banach extension property, 25 (1970), 902-905.

46A15

Shapiro, Leonard D.

1 Proximality in minimal transformation groups, 26 (1970), 521-525.

$54 \mathrm{H} 20$; 54G20 54H15

Sharp, Henry S.

1 The permanent of a transitive relation, 26 (1970), 153-157.

Shatz, Stephen S.

04A05 05A10 05B20; 04A15 28A05 54F99 54H05

1 Principal homogeneous spaces for finite group schemes, 22 (1969), 678-680.

Shawyer, Bruce Lockhart Robertson

$14 \mathrm{~L} 20$

1 On the relation between the Abel and Borel-type methods of summability, 22 (1969), 15-19.

40D25 40G10

2 (with Yang, Gou-sheng) On the relation between the Abel-type and Borel-type methods of summability, 26 (1970), 323-328. 40D25 40G10

Shea, Daniel F.

1 On a complement to Valiron's tauberian theorem for the Stieltjes transform, 21 (1969), 1-9.

40E05 44A 15

Sher, R. B.

1 Determining the cellularity of a 1-complex by properties of its arcs, 26 (1970), 491-498.

57A15 57A60; 55A15

2 Tame polyhedra in wild cells and spheres, 30 (1971), 169-174.

57A15 57A45 57C05 57C55; 55A30 57A60

Sherman, Thomas $\mathbf{L}$.

1 On a theorem of Azbelev and Caljuk, 21 (1969), 63. Shiffman, Bernard

1 Local complex analytic curves in an analytic variety, 24 (1970), 432-437.

Shore, Samuel D.

1 Decomposition of function-lattices, 28 (1970), 189-190.

Shores, T. S.

1 Decompositions of finitely generated modules, 30 (1971), 445-450.

$13 \mathrm{C} 05$ 13C10; $13 \mathrm{~F} 05$

Shub, Michael

1 (with Williams, Robert F.) Future stability is not generic, 22 (1969), 483-484.

Sibuya, Yasutaka

$58 \mathrm{~F} 10$

1 Almost periodic solutions of Poisson's equation, 28 (1971), 195-198.

31B35 35B25 35J05 42A84; 31B05 35D99 46H99 
Sidney, S. J.

1 An example concerning core measures, 26 (1970), 428-430. 46J10

2 Peak points for hypo-Dirichlet algebras, 26 (1970), 431-436. $46 \mathrm{~J} 10$ Siegel, Jerrold

$1 k$-invariants in local coefficient theory, 29 (1971), 169-174.

55B25 55F15 55F35 55G45; 55H 10

Sieradski, Allan J.

1 Generalized theories for k-spaces, 24 (1970), 52-56.

54D50 55B20

2 An example of Hilton and Roitberg, 28 (1971), 247-253.

55D10 55D15 55F25; 55D45

Sigillito, Vincent G.

1 On the uniqueness of solutions of certain improperly posed problems, 24 (1970), 828-831.

35L05 35R25; 35A05

Sikkema, Carl D.

1 A duality between spheres and spheres with arcs, 25 (1970), 781-785.

57A10; 54A05

Silberger, Allan J.

1 All algebras of spherical functions defined on the two-by-two general linear group with entries in a locally compact p-adic field are commutative, 21 (1969), 437-440.

22E35

Simon, Hermann

1 Extensions of torsionfree groups by torsion groups, 23 (1969), 433-438. 20F25 Simons, F. H.

1 Recurrence and preservation of measure, 24 (1970), $221 . \quad 28 \mathrm{~A} 65$ Sinclair, Allan M.

1 Jordan automorphisms on a semisimple Banach algebra, 25 (1970), 526-528.

2 The norm of a hermitian element in a Banach algebra, 28 (1971), 446-450.

46H99; 30A42 46K99 47A10

3 (with Knopfmacher, J.) Fields with few extensions, 29 (1971), 255-258. $10 \mathrm{~J} 10$ Sine, R. C.

1 Smoothing in $C(X), 21$ (1969), 490-492.

$46 \mathrm{E} 15$

$2 A$ note on rays at the identity operator, 23 (1969), 546-547. 47D20

3 A mean ergodic theorem, 24 (1970), 438-439.

$28 \mathrm{~A} 65$

Singer, Ivan

1 Remark on a paper of Y. Ikebe, 21 (1969), 24-26. 41A65

Singh, Sheo Ram

1 On the absolute Nörlund summability factors of infinite series, 25 (1970), 684-689. 40F05 40G05

Sinkhorn, Richard

1 Concerning a conjecture of Marshall Hall, 21 (1969), 197-201. 15A15 15A51

2 Linear transformations under which the doubly stochastic matrices are invariant, 27 (1971), 213-221.

15A51 15A72; 15A21

Siromoney, Rani

1 A characterization of semilinear sets, 21 (1969), 689-694.

94A30

Sitaramachandra Rao, $\mathbf{R}$.

1 (with Suryanarayana, D.) On the order of the error function of the $k$-free integers, 
28 (1971), 53-58.

$10 \mathrm{H} 15$

Siu, Yum-tong

1 Sheaf cohomology with bounds and bounded holomorphic functions, 21 (1969), 226-229.

$32 \mathrm{C} 35 ; 32 \mathrm{~A} 07$

2 Noetherianness of rings of holomorphic functions on Stein compact subsets, 21 (1969), 483-492.

$32 \mathrm{C} 15$

\section{Skerry, Herbert}

1 (with Jakimovski, Amnon) Some regularity conditions for the $\left(f, d_{n}, z_{1}\right)$ summability method, 24 (1970), 281-287. 40C05 40D05 40G99

Skoug, D. L.

1 (with Johnson, Gerald W.) Operator-valued Feynman integrals of certain finite dimensional functionals, 24 (1970), 774-780.

28A40

2 (with Johnson, Gerald W.) An operator valued function space integral: a sequel to Cameron and Storvick's paper, 27 (1971), 514-518.

28A40

Skwarczyński, M.

1 The invariant distance in the theory of pseudo-conformal transformations and the $L u$ Qi-Keng conjecture, 22 (1969), 305-310. 30A30 30A31 32H10 32H15

\section{Slivka, John}

1 (with Severo, Norman C.) On the strong law of large numbers, 24 (1970), 729-734. 60F10 60F15 62E20; 60E05 60F05 60F20

\section{Slover, Rebecca Ellen}

1 (with Fletcher, Peter and McCoy, R. A.) On boundedly metacompact and boundedly paracompact spaces, 25 (1970), 335-342.

54D15 54D20 54F45

Smith, James C., Jr.

1 Lebesgue characterizations of uniformity-dimension functions, 22 (1969), 164-169.

Smith, John H.

54F45; 54E15 54E35

1 A result of Bass on cyclotomic extension fields, 24 (1970), 394-395. $\quad$ 12A35

Smith, Larry

1 (with Smith, Mi-Soo Bae) On the cohomology Chern classes of the K-theory Chern classes, 26 (1970), 209-214. 55B15 55F25 55F50 57D20; 55F40

Smith, Mi-Soo Bae

1 (with Smith, Larry) On the cohomology Chern classes of the $K$-theory Chern classes, 26 (1970), 209-214. 55B15 55F25 55F50 57D20; 55F40

Smith, William W.

1 A covering condition for prime ideals, 30 (1971), 451-452.

$13 \mathrm{~A} 15$

Smithson, Raymond E.

$1 A$ note on $\delta$-continuity and proximate fixed points for multi-valued functions, 23 (1969), 256-260.

$54 \mathrm{C} 60$ 54H25; 54F50

2 Fixed points for contractive multifunctions, 27 (1971), 192-194.

$54 \mathrm{C} 30$ 54H25; 54B20 54E35

3 Fixed points of order preserving multifunctions, 28 (1971), 304-310.

06A10 54C60 54F05 54H25; 54C65

4 (with George, John H. and Sehgal, V. M.) Application of Liapunov's direct method to fixed point theorems, 28 (1971), 613-620. 54C60 54H25; 54E35 54E45 Smythe, N.

1 Handlebodies in 3-manifolds, 26 (1970), 534-538. 
Soare, Robert I.

1 (with Jockusch, Carl G., Jr.) Minimal covers and arithmetical sets, 25 (1970), 856-859.

Solitar, Donald

1 (with Karrass, Abraham) On finitely generated subgroups of a free group, 22 (1969), 209-213.

2 (with Karrass, Abraham) On groups with one defining relation having an abelian normal subgroup, 23 (1969), 5-10.

20K05

3 (with Baumslag, Gilbert and Karrass, Abraham) Torsion-free groups and amalgamated products, 24 (1970), 688-690.

4 (with Karrass, Abraham) On the free product of two groups with an amalgamated subgroup of finite index in each factor, 26 (1970), 28-32. 20E05 20E30

Sommese, Joseph E.

1 On a maximal ideal space separated by a peak point, 26 (1970), 471-472.

Sparks, Arthur G.

1 Intersections of maximal $L_{n}$ sets, 24 (1970), 245-250. Spatz, I. N.

1 Smooth Banach algebras, 22 (1969), 328-329. 46 H99

Speers, Richard

1 Jordan structures in simple graded rings, 24 (1970), 22-23. 16A68

Sperry, P. L.

1 On generating systems for abelian groups, 24 (1970), 148-153. 20K99

Spira, Robert

1 Another zero-free region for $\zeta^{(k)}(s), 26$ (1970), 246-247.

$10 \mathrm{HOS}$

Srivastava, H. M.

1 Generating functions for Jacobi and Laguerre polynomials, 23 (1969), 590-595.

Stackelberg, Olaf P.

$33 \mathrm{~A} 65$

1 An upper asymptotic estimate of Brownian path variation, 26 (1970), 168-173.

Stanford, David P.

60F15 60J65

1 Boundedness and dimension for weighted average functions, 24 (1970), 82-84.

Stark, H. M.

1 A historical note on complex quadratic fields with class-number one, 21 (1969), 254-255.

12A25 12A50

Staudte, Robert G., Jr.

1 (with Tata, Mahabanoo N.) Complex roots of real characteristic functions, 25 (1970), 238-246.

60E05 62E10; 60B 15

Stavrakas, Nick M.

1 (with Hare, William R. and Kenelly, J. W.) Two cells with $N$ points of local nonconvexity, 27 (1971), 331-336.

52A10

Stebe, Peter

1 A residual property of certain groups, 26 (1970), 37-42.

20E25 20F 10 Steen, Lynn A. 
1 A direct proof that a linearly ordered space is hereditarily collection wise normal, 24 (1970), 727-728.

54F05; 54D15

Stein, F. Max

1 (with Henry, Myron S.) An approximate solution of the Riccati matrix equation, 25 (1970), 8-12.

$34 \mathrm{~A} 45$

Stein, George $\mathbf{H}$.

1 Entropy and density, 28 (1971), 505-508. 28A70

Stein, James D., Jr.

1 Several theorems on boundedness and equicontinuity, 26 (1970), 415-419.

Steiner, A. $\mathbf{K}$.

46B05

1 (with Steiner, Eugene F.) Graph closures and metric compactifications of $N, 25$ (1970), 593-597.

54D35 54D40; 54D45

2 On the topological completion of M-space products, 29 (1971), 617-620.

54D20 54G20; 54B10 54C10 54E15

Steiner, Eugene F.

1 (with Steiner, A. K.) Graph closures and metric compactifications of N, 25 (1970), 593-597.

54D35 54D40; 54D45

Stephenson, Robert M., Jr.

1 Product spaces for which the Stone-Weierstrass theorem holds, 21 (1969), 284-288. 54C40 54D10 54D15 54D30; 54D10 54D25 54D50

2 A countable minimal Urysohn space is compact, 22 (1969), 625-626.

54D25; 54D30

3 Noncut points and modified compactness conditions, 23 (1969), 266-272.

$54 \mathrm{~F} 15 ; 54 \mathrm{D} 25$

4 (with Saks, Victor) Products of $\mathfrak{M}$-compact spaces, 28 (1971), 279-288.

Stepp, James W.

54B10 54D30; 54A25 54D35

1 D-semigroups, 22 (1969), 402-406.

22A 15

2 Semilattices which are embeddable in a product of min intervals, 28 (1971), 81-86.

Stewart, D. G.

06A20; 20M10 22A15

1 (with Anderson, Bruce A.) $T_{1}$-complements of $T_{1}$ topologies, 23 (1969), 77-81.

Stitzinger, Ernest L.

54A10; 06A25

1 A nonembedding theorem for finite groups, 25 (1970), 124-126. 20D25

2 On elementary groups, 26 (1970), 236-238.

20D25

3 Minimal nonnilpotent solvable Lie algebras, 28 (1971), 47-49.

St. Mary, Donald F.

17B05 17B30; 20F40

1 (with Fink, A. M.) On an inequality of Nehari, 21 (1969), 640-642. 34C10 Stoddart, A. W. J.

1 An extension of the Thomson principle, 29 (1971), 561-564. 26A84; 31A15 Stoll, Wilhelm

1 About the universal covering of the complement of a complete quadrilateral, 22 (1969), 326-327.

$32 \mathrm{C} 25$

2 The construction of a $\bar{\partial}$-simple covering, 27 (1971), 101-106. 
Stoltenberg, Ronald A.

$32 \mathrm{C} 10$ 32L10; 55B30

1 A note on stratifiable spaces, 23 (1969), 294-297.

54E20; 54A25 54C10

Stone, A. H.

1 (with ErdõS, Paul) On the sum of two Borel sets, 25 (1970), 304-306.

04A15 26A21 28A05 54C50

Stone, Anthony $\mathbf{P}$.

1 (with Blair, David E.) A note on the holonomy group of manifolds with certain $\begin{array}{ll}\text { structures, } 21 \text { (1969), 73-76. 53B20 } & \end{array}$

Stout, E. L.

1 Riesz and Laurent decompositions on Riemann surfaces, 24 (1970), 324-331.

30A76; 30A46 30A88

Strait, Peggy Tang

1 On Berman's version of the Lévy-Baxter theorem, 23 (1969), 91-93.

60F15 60J65; 60C05 60G15 60J30

2 A flat integral for functionals defined on sample paths of a Brownian process with the parameter in N-dimensional space, 25 (1970), 21-23. $60 \mathrm{H05} 60 \mathrm{J65}$; 47B10

Stratton, Howard H., Jr.

1 (with Brown, Herbert I. and Kerr, Donald R., Jr.) The structure of $B[c]$ and extensions of the concept of conull matrix, 22 (1969), 7-14. 46L20; 40H05

2 (with Brown, Herbert I.) Conullity of operators on some FK-spaces, 25 (1970), 717-727.

40H05 40J05 46A05 46A45

Straus, Ernst G.

1 (with Hilliker, D. L.) Some p-adic versions of Polya's theorem on integer valued analytic functions, 26 (1970), 395-400.

12B05

Strauss, Aaron

1 (with Yorke, James A.) Identifying perturbations which preserve asymptotic stability, 22 (1969), 513-518.

34D10

2 (with Yorke, James A.) Linear perturbations of ordinary differential equations, 26 (1970), 255-260.

34D10

Stecker, G. E.

1 (with Viglino, Giovanni) Cotopology and minimal Hausdorff spaces, 21 (1969), 569-574.

54A10 54D25; 54D10 54D35

Strichartz, Robert S.

1 A multilinear version of the Marcinkiewicz interpolation theorem, 21 (1969), 441444.

$46 \mathrm{E} 35$

2 A note on Sobolev algebras, 29 (1971), 205-207.

$46 \mathrm{E} 35 ; 46 \mathrm{~J} 10$

Stringall, Robert $\mathbf{W}$.

1 Decompositions of Abelian p-groups, 28 (1971), 409-410.

06A40 20K10

2 The categories of p-rings are equivalent, 29 (1971), 229-235.

06A40; 02J05 16A30

Subbarao, M. V.

1 (with Vidyasagar, M.) On Watson's quintuple product identity, 26 (1970), 23-27;

Erratum: 29 (1971), 627.

05A 19; 05A 15

2 On a partition theorem of MacMahon-Andrews, 27 (1971), 449-450. 10 A45

3 Partition theorems for Euler pairs, 28 (1971), 330-336. 10A45

Suffiridge, Ted J.

1 On the kernel function for the intersection of two simply connected domains, 22 
(1969), 37-41.

$30 \mathrm{~A} 31$

Summerhill, Ralph R.

1 Tree-like continua and cellularity, 26 (1970), 201-205.

54C25 54F20 54F50; 54F15 54F60

Sumners, D. W.

$1 \mathrm{H}_{2}$ of the commutator subgroup of a knot group, 28 (1971), 319-320.

Summers, W. H.

$18 \mathrm{H} 10$ 55A05 55A25

1 (with Collins, Heron Sherwood) Some applications of Hewitt's factorization theorem, 21 (1969), 727-733.

$46 \mathrm{H} 05$ 46H25; 47B05

Summers, D. W. L.

1 Homotopy torsion in codimension two knots, 24 (1970), 229-240.

55A05 57C45

\section{Sundaresan, Kondagunta}

1 Extreme points of the unit cell in Lebesgue-Bochner function spaces. I, 23 (1969), 179-184; Erratum: 25 (1970), 922.

46E30 46E40

Suryanarayana, $\mathbf{D}$.

1 (with Sitaramachandra Rao, R.) On the order of the error function of the $k$-free integers, 28 (1971), 53-58. $10 \mathrm{H} 15$

Sutton, W. G.

1 (with George, John H.) Application of Liapunov theory to boundary value problems, 25 (1970), 666-671.

34B 15

Suzuki, Noboru

1 The structure of spectral operators with completely continuous imaginary part, 22 (1969), 82-84.

47A65 47B40

2 On the irreducibility of weighted shifts, 22 (1969), 579-581.

47B20

3 On a weakly convergent sequence of normal functionals on a von Neumann algebra, 22 (1969), 697-701.

$46 \mathrm{~L} 10$

Swan, Richard G.

1 Correction to 'An application of graph theory to algebra', 21 (1969), 379-380.

$05 \mathrm{C} 3015 \mathrm{~A} 06$

\section{Swanson, C. A.}

1 Oscillation criteria for nonlinear matrix differential inequalities, 24 (1970), 824-827.

$34 \mathrm{C} 15$

2 (with Allegretto, Walter) Oscillation criteria for elliptic systems, 27 (1971), 325-330.

35B05 $35 \mathrm{~J} 60$

Swetits, John

1 Summability of a Cauchy product series, 23 (1969), 144-146. $\quad$ 40C15 40D99

Tam, K. W.

1 On measures with separable orbit, 23 (1969), 409-411.

43A05

Tamano, Hisahiro

1 (with Vaughan, J. E.) Paracompactness and elastic spaces, 28 (9171), 299-303.

Targonski, György I.

1 (with Schreiber, Michel) Carleman and semi-Carleman operators, 24 (1970), 293 299.

$47 \mathrm{G05}$

Tarsy, Richard B.

1 Global dimension of triangular orders, 28 (1971), 423-426. 18G20; 16A62 
Tarwater, J. Dalton

1 Homogeneous primary abelian groups, 24 (1970), 154-155. 20K30 Tata, Mahabanoo N.

1 (with Staudte, Robert G., Jr.) Complex roots of real characteristic functions, 25 (1970), 238-246. 60E05 62E10; 60B15

Taylor, B. A.

1 (with Williams, D. L.) The peak sets of $A^{m}, 24$ (1970), 604-606. 30A42 Taylor, Gerald D.

1 (with Salehi, Habib) Positive matrix $H^{1 / 2}$ and Hermitian matrix $H^{1}$ functions are $\begin{array}{ll}\text { constant, } 26 \text { (1970), 469-470. 30A78; } 15 \mathrm{~A} 48 & \end{array}$

Taylor, Michael E.

1 Analytic properties of elliptic and conditionally elliptic operators, 28 (1971), 317-318.

Teply, Mark L.

$35 \mathrm{~B} 4535 \mathrm{~J} 30$

1 Torsionfree projective modules, 27 (1971), 29-34.

16A50; 16A08 16A62 18G05

2 A note on modules over a commutative regular ring, 29 (1971), 267-268.

Terkelsen, Frode

13C10 16A30; $18 \mathrm{E} 40$

1 On semigroups of operators in locally convex spaces, 22 (1969), 340-343.

Thedy, Armin

47D05

1 On rings satisfying $[(a, b, c), d]=0,29(1971), 250-254 . \quad$ 17A30 17E05

Thie, Paul R.

1 The area of an analytic set in complex projective space, 21 (1969), 553-554.

$32 \mathrm{C} 2532 \mathrm{C} 30$

2 The Lelong number of a complete intersection, 24 (1970), 319-323. 32C25 Thomas, C. B.

1 On periodic maps which respect a symplectic structure, 22 (1969), 251-254.

Thomas, James W.

55B15 55C35 57D90

1 (with Sehgal, V. M.) A fixed point theorem for semigroups of mappings with a contractive iterate, 29 (1971), 565-568.

47D05 47H10

Thomason, S. K.

1 A proof of Whitman's representation theorem for finite lattices, 25 (1970), 618-619.

Thompson, Gerald L.

06A20; 08A05

1 (with Weil, Roman L., Jr.) Reducing the rank of $(A-\lambda B), 26$ (1970), 548-554.

Tillman, Stephen J.

15A03; 15A 18 15A 30

1 The multiplicative group of absolutely algebraic fields in characteristic p, 23 (1969), 601-604.

$12 C 30$

Tindell, Ralph S.

1 Extending homeomorphisms of $S^{p} \times S^{q}, 22$ (1969), 230-232. 57C99; 57C25

2 Noncombinatorial triangulations and the Poincaré conjecture, 24 (1970), 60-62.

Ting, Wei-lung 
1 On cohomology groups of Banach algebras, 21 (1969), 175-178. 46J99 46M99 Tirao, Juan A.

1 Selfadjoint function spaces on Riemannian symmetric manifolds, 24 (1970), 223228.

To, Ting-on

1 The equivalence of the least upper bound property and the Hahn-Banach extension property in ordered linear spaces, 30 (1971), 287-295. 46A40; 06A20

\section{Tollefson, Jefferey $\mathbf{L}$.}

1 3-manifolds fibering over $S^{1}$ with nonunique connected fiber, 21 (1969), 79-80.

55F55 57A10; 20E40 55A05

2 Imbedding free cyclic group actions in circle group actions, 26 (1970), 671-673. 57E10 57E30; 57A10

3 (with Rogers, James Ted, Jr.) Homeomorphism groups of weak solenoidal spaces, 28 (1971), 242-246.

54B25 54H15; 57A 15

4 (with Rogers, James Ted, Jr.) Homogeneous inverse limit spaces with nonregular covering maps as bonding maps, 29 (1971), 417-420. $\quad$ 54B25 57A15; 54G20

Tomiuk, Bohdan J.

1 (with Wong, Pak-ken) The Arens product and duality in $B^{*}$-algebras, 25 (1970), 529-535.

46H99 46L05

Tondra, Richard J.

1 Characterization of connected 2-manifolds without boundary which have finite domain rank, 22 (1969), 479-482.

57A05

2 Surfaces of finite domain rank, 26 (1970), 181-184.

55A20 55A99

3 The domain rank of open surfaces of infinite genus, 28 (1971), 581-583.

Tong, Alfred E.

55A30 55A99 57A05

1 Projecting the space of bounded operators onto the space of compact operators, 24 (1970), 362-365.

46A45; 47B05

Tonne, Philip C.

1 On the convergence of Bernstein polynomials for some unbounded analytic functions, 22 (1969), 1-6.

41A10; 40A05

Torrance, Ellen

1 Maximal C*-subalgebras of a Banach algebra, 25 (1970), 622-624.

46H10 46LO5

2 Strictly convex spaces via semi-inner-product space orthogonality, 26 (1970), 108110.

46B99

Transue, William R. R.

1 (with Bennett, Ralph) On embedding cones over circularly chainable continua, 21 (1969), 275-276.

54C20 54F20; 54F55 57A05 57A10 57A15 57A35

2 (with Hinrichsen, J. W. and Fitzpatrick, Ben, Jr.) Concerning upper semicontinuous decompositions of irreducible continua., 30 (1971), 157-163. 54F20; 54B15

Trotter, Hale F.

1 On the norms of units in quadratic fields, 22 (1969), 198-201. 12A25 12A45

Trotter, William T., Jr.

1 Characterization of the finite partition property for a collection of universal subcontinua, 25 (1970), 760-762.

Tsagas, Grigorios

54F15; 54F 20

1 A relation between killing tensor fields and negative pinched Riemannian manifolds, 
22 (1969), 476-478.

53C20 57D25

Tsai, Chester E.

1 The Levitzki radical in Jordan rings, 24 (1970), 119-123. 17C10

Tse, Kam-fook

1 An analog of the Lusin-Privaloff radial uniqueness theorem, 25 (1970), 310-312.

$30 \mathrm{~A} 72$

2 Nontangential interpolating sequences and interpolation by normal functions, 29 (1971), 351-354.

30A74 30A80; 30A76

Tserpes, Nicholas

1 (with Mukherjea, Arunava) Idempotent measures on locally compact semigroups, 29 (1971), 143-150.

28A70 43A05 60B15; 43A07 60B05

Turakainen, Paavo

1 Generalized automata and stochastic languages, 21 (1969), 303-309.

Turdza, Erwin

94A30 94A35

1 On the stability of the functional equation $\phi[f(x)]=g(x) \phi(x)+F(x), 30(1971)$, 484-486.

39A 15

Turnidge, Darrell R.

1 Torsion theories and semihereditary rings, 24 (1970), 137-143. 16A50; 16A30 Twomey, J. B.

1 On starlike functions, 24 (1970), 95-97.

$30 \mathrm{~A} 32$

2 (with Saff, E. B.) A note on the location of critical points of polynomials, 27 (1971), 303-308.

30A08

Tzafriri, $\mathbf{L}$.

1 Reflexivity of cyclic Banach spaces, 22 (1969), 61-68.

46B 10; 46A40

2 Conditional expectations and an isomorphic characterization of $L_{1}$-spaces, 27 (1971), 317-324. 46B99 46E30

Uchiyama, Saburô

1 On some products involving primes, 28 (1971), 629-630. 10H25 57A99 Uhl, J. J., Jr.

1 The Radon-Nikodym theorem and the mean convergence of Banach space valued martingales, 21 (1969), 139-144.

28A10 28A25 28A45 46G10 60G45; 28A15 46B10 46G05 47D99

2 The range of a vector-valued measure, 21 (1969), 158-163.

28A45

3 Abstract martingales in Banach spaces, 28 (1971), 191-194.

46G10; 60G45

Ulrich, Dolph

1 Solution to a problem posed by Kalicki, 22 (1969), 728-729.

$02 \mathrm{C05}$

Unger, Gerald S.

1 Completely regular maps, fiber maps and local n-connectivity, 21 (1969), 104108.

54D05 55F05

Utz, W. Roy

1 Oscillating solutions of third order differential equations, 26 (1970), 273-276.

Valentine, Joseph E.

1 Hyperbolic spaces and quadratic forms, 24 (1970), 607-610. 50C05 Vanden Eynden, C. L.

1 (with Crittenden, Richard B.) Any $n$ arithmetic progressions covering the first $2^{n}$ integers cover all integers, 24 (1970), 475-481.

$10 \mathrm{~A} 10$ 
Van der Poorten, A. J.

1 On the arithmetic nature of definite integrals of rational functions, 29 (1971), 451-456.

Van de Water, Arthur

1 A property of torsion-free modules over left Ore domains, 25 (1970), 199-201.

Van Vleck, F. S.

16A02 16A52

1 (with Himmelberg, Charles J. and Porter, Jack R.) Fixed point theorems for condensing multifunctions, 23 (1969), 635-641.

$47 \mathrm{H} 10$ Varadarajan, $\mathbf{K}$.

1 A note on topological parallelizability, 22 (1969), 607-609. $\quad$ 57A55 57E05 Vasconcelos, Wolmer V.

1 On projective modules of finite rank, 22 (1969), 430-433.

13C10; 14A99

2 Injective endomorphisms of finitely generated modules, 25 (1970), 900-901.

13C05 13C15 13C99 13E10; 13A99 13D99

3 Annihilators of modules with a finite free resolution, 29 (1971), 440-442.

Vasquez, Alphonse T.

13C10; 13D05

1 (with Lazarov, Connor) The difference construction in K-theory, 21 (1969), 315 317.

Vaughan, J. E.

22C05 55B15; 22D12 81A78

1 Linearly ordered collections and paracompactness, 24 (1970), 186-192.

54D20; 54D10 54D15 54E35

2 (with Tamano, Hisahiro) Paracompactness and elastic spaces, 28 (9171), 299-303.

Vaughan, Loy Ottis, Jr.

54D20 54E20 54E35

1 (with Gray, William J.) The almost fixed point property for hereditarily unicoherent continua, 27 (1971), 381-386. 54C60 54H25; 54F50

Veech, William A.

1 Short proof of Sobczyk's theorem, 28 (1971), 627-628.

46B99; 46E15

Venkatanarasimhan, P. V.

1 Pseudo-complements in posets, 28 (1971), 9-17. 06 A10; 06A20 06A40 Verhey, Roger F.

1 (with Marx, Morris L.) Interior and polynomial extensions of immersed circles, 24 (1970), 41-49.

30A90

Verma, Arun

1 (with Al-Salam, Waleed A.) Some orthogonality preserving operators, 23 (1969), 136-139.

Vick, James W.

42A52; 34A35

1 Pontryagin duality in K-theory, 24 (1970), 611-616.

Vidossich, Giovanni

55B15 55F40; $18 \mathrm{H} 1020 \mathrm{C} 15$

1 A remark on the density character of function spaces, 22 (1969), 618-619.

54A25 54C $35 ; 46 \mathrm{~A} 05$

2 A note on cardinal reflections in the category of uniform spaces, 23 (1969), 55-58.

54E15

3 Uniform spaces of countable type, 25 (1970), 551-553.

54D20 54E15 
4 Topological characterizations of pseudo- $\boldsymbol{N}$-compact spaces, 27 (1971), 195-198. 54D20 54D30 54E15; 54D35

Vidyasagar, $\mathbf{M}$.

1 (with Subbarao, M. V.) On Watson's quintuple product identity, 26 (1970), 23-27; Erratum: 29 (1971), 627.

05A $19 ; 05 \mathrm{~A} 15$

Viglino, Giovanni

1 (with Stecker, G. E.) Cotopology and minimal Hausdorff spaces, 21 (1969), 569-574.

Vilms, Jaak

54A10 54D25; 54D 10 54D35

1 Nonlinear and direction connections, 28 (1971), 567-572.

53B15 53B40

Vinograde, Bernard

1 (with Mordeson, John N.) Note on relative P-bases of purely inseparable extensions, 22 (1969), 587-590.

Viswanathan, T. M.

1 (with Reis, Clive M.) A compactness property for prime ideals in Noetherian rings, 25 (1970), 353-356.

$13 \mathrm{~A} 15 ; 13 \mathrm{C} 99$

Vučković, Vladeta

1 Almost recursive sets, 23 (1969), 114-119.

$02 \mathrm{~F} 25$

Wade, William $\mathbf{R}$.

1 Summing closed U-sets for Walsh series, 29 (1971), 123-125. $\quad$ 42A52 42A56

Waelbroeck, Lucien

1 Differentiability of Hölder-continuous semigroups, 21 (1969), 451-454. 47D05

Wagoner, Ronald $\mathbf{L}$.

1 Cogenerator endomorphism rings, 28 (1971), 347-351.

\section{Walker, Elbert A.}

16A50 16A52; 16A64 18G05

1 (with Richman, Fred) Extending Ulm's theorem without group theory, 21 (1969), 194-196.

04A20 20K25

Walker, Homer E.

1 On the null-spaces of first-order elliptic partial differential operators in $R^{n}, 30$ (1971), 278-286.

Walkup, David W.

1 (with Wets, Roger J.-B.) A Lipschitzian characterization of convex polyhedra, 23 (1969), 167-173.

$52 \mathrm{~A} 25$

Wallach, Nolan $R$.

1 Induced representations of Lie algebras. II, 21 (1969), 161-166. 17B10 17B20

2 (with Warner, Frank W.) Curvature forms for 2-manifolds, 25 (1970), 712-713.

$53 \mathrm{C} 20 ; 58 \mathrm{~A} 10$

Wallen, Lawrence J.

1 Fourier-Stieltjes transforms tending to zero, 24 (1970), 651-652. 42A16 42A68 Walsh, B. J.

1 Mutual absolute continuity of sets of measures, 29 (1971), 506-510.

28A10 28A45 46G10; 46A05 47B99

Walsh, $\mathbf{T}$.

1 On the existence of double singular integrals for kernels without smoothness, 28 (1971), 439-445.

44A25; 47G05

Waltman, Paul E.

1 (with Schrader, Keith W.) An existence theorem for nonlinear boundary value problems, 21 (1969), 653-656. 
Wamsley, J. W.

1 The deficiency of metacyclic groups, 24 (1970), 724-726.

20D10 20F05

Wang, Ju-kwei

1 Note on a theorem of Nehari on Hankel forms, 24 (1970), 103-105.

Wang, S. P.

43A25; 22B99

1 On the centralizer of a lattice, 21 (1969), 21-23.

22E40

2 On a conjecture of Chabauty, 23 (1969), 569-572.

22E40; $10 \mathrm{E} 05$

3 On a theorem of representation of lattices, 23 (1969), 583-587.

22E40 22E45

Ward, L. E., Jr.

1 (with Nadler, Sam B., Jr.) Concerning continuous selections, 25 (1970), 369-374. 54C65; 54F50

2 Arcs in hyperspaces which are not compact, 28 (1971), 254-258.

54B20 54B20 54F05 54F05; 54D05

Ware, Roger

1 (with Zelmanowitz, Julius Martin) The Jacobson radical of the endomorphism ring of a projective module, 26 (1970), 15-20. 16A21 16A42;16A50

Warfield, R. B., Jr.

1 (with McShane, Edward James) Addenda and corrigenda to 'On Filippov's implicit functions lemma', 21 (1969), 496-498.

26A57

2 A Krull-Schmidt theorem for infinite sums of modules, 22 (1969), 460-465.

16A64; 16A10 16A46 16A50

3 Decomposability of finitely presented modules, 25 (1970), 167-172.

Warner, Frank W.

$13 \mathrm{C} 05 ; 13 \mathrm{~A} 15$

1 (with Wallach, Nolan R.) Curvature forms for 2-manifolds, 25 (1970), 712-713.

Warner, Kenneth K.

53C20; 58A 10

1 The spectrum of a linear operator under perturbation by certain compact operators, 22 (1969), 667-671.

47A10 47A55

2 A note on a theorem of Weyl, 23 (1969), 469-471.

47A55; 47B20

Warner, Seth

1 Metrizability of locally compact vector spaces, 27 (1971), 511-513.

$16 \mathrm{~A} 8022 \mathrm{~A} 30$

2 Sheltered modules and rings, 30 (1971), 8-14.

13A15 16A80; 13J99 16A64

Warren, Hugh $E$.

1 A construction of certain nonlinear approximating families, 21 (1969), 467-470.

2 A special basis for $C([0,1]), 27$ (1971), 495-499.

$41 \mathrm{~A} 1041 \mathrm{~A} 63$

3 A Riemann mapping theorem for $C(X), 28$ (1971), 147-154.

46B $15 ; 46 \mathrm{E} 15$

ashenberger, James $\mathrm{K}$.

$46 \mathrm{~J} 10 ; 30 \mathrm{~A} 30$

1 Fredholm operators: a counterexample, 21 (1969), 739-740. 47B30; 46A05

Waterman, Alan G.

1 The normal completions of certain partially ordered vector spaces, 25 (1970), 141- 
Wayment, S. G.

1 (with Cannon, J. W.) An imbedding problem, 25 (1970), 566-570.

54A20 54C25 54F15 57A10 57A15; 54C35 57A05

2 On congruence indices for simple closed curves, 28 (1971), 199-207.

52A10 52A50

3 (with Edwards, J. R.) A v-integral representation for linear operators on a space of continuous vector valued functions, 30 (1971), 260-262.

28A45 46E 10

4 (with Edwards, J. R.) $A$ v-integral representation for the continuous linear operators on spaces of continuously differentiable vector-valued functions, 30 (1971), 263270.

$28 \mathrm{~A} 45$ 46E10

Weaver, J. R.

1 Solvable automorphism groups and an upper bound for $|A(G)|, 27$ (1971), 229-235.

Webb, William A.

1 On $^{\circ} 4 / n=1 / x+1 / y+1 / z, 25(1970), 578-584 . \quad 10 \mathrm{~B} 10$

Weddington, D. D.

1 (with Bagley, Robert W.) Products of $k^{\prime}$-spaces, 22 (1969), 392-394. $\quad$ 54D50

2 On $k$-spaces, 22 (1969), 635-638. 54D50

Weil, Roman L., Jr.

1 (with Thompson, Gerald L.) Reducing the rank of $(A-\lambda B), 26$ (1970), 548-554.

Weinbaum, C. $\mathbf{M}$.

15A03; 15A 18 15A 30

1 The word and conjugacy problems for the knot group of any tame, prime, alternating knot, 30 (1971), 22-26.

20E40 20F05 20F10 55A25; 55A05

Weinstein, Alan D.

1 Positively curved deformations of invariant Riemannian metrics, 26 (1970), 151-152.

53C20; 53C 30

2 (with Marsden, Jerrold E.) A comparison theorem for Hamiltonian vector fields, 26 (1970), 629-631.

$34 \mathrm{C} 35$

Weinstock, Barnet $M$.

1 Continuous boundary values of analytic functions of several complex variables, 21 (1969), 463-466.

32D10 32F 15

2 An approximation theorem for $\bar{\partial}$-closed forms of type ( $n, n-1), 26$ (1970), 625-628.

Weisman, Carl S.

1 On the connected identity component of the Adèle-class group of an algebraic torus, 21 (1969), 155-160.

20G30 20G35

\section{Weiss, Max L.}

1 (with Boehme, T. K.) One-sided boundary behavior for certain harmonic functions, 27 (1971), 280-288.

$31 \mathrm{~A} 20$

2 (with Boehme, T. K.) Extensions of Fatou's theorem to tangential asymptotic values, 27 (1971), 289-298.

30A98 31A20

Weissglass, Julian

1 Regularity of semigroup rings, 25 (1970), 499-503. 16A26 16A30 20M30

Weissner, Edward W.

1 (with Leadbetter, M. R.) On continuity and other analytic properties of stochastic process sample functions, 22 (1969), 291-294.

60G15 60G17 


\section{Weitsman, Allen}

1 A growth property of the Nevanlinna characteristic, 26 (1970), 65-70.

30A68 30A70

Wells, Benjamin B., Jr.

1 A random $L^{1}$ function with divergent Walsh series, 24 (1970), 794-796.

Wend, David V. V.

$42 A 56$

1 Existence and uniqueness of solutions of ordinary differential equations, 23 (1969), 27-33.

$34 \mathrm{~A} 10$

2 (with Ryder, Gerald H.) Oscillation of solutions of certain ordinary differential equations of nth order, 25 (1970), 463-469. $34 \mathrm{C10}$

Wenner, B. R.

1 Dimension-theoretic properties of completions, 28 (1971), 590-594.

West, James E.

54F45; 54C20 54D35 54E35

1 Fixed-point sets of transformation groups on infinite-product spaces, 21 (1969), 575-582.

54H15 54H25; 54B 10

Westman, Joel J.

1 Cohomology for the ergodic actions of countable groups, 30 (1971), 318-320.

$18 \mathrm{H} 40$ 22D40 28A65

Weston, J. H.

1 (with Gulden, Samuel L. and Fleischman, W. M.) Linearly ordered topological spaces, 24 (1970), 197-203.

54D20 54F05; 06A45 54A25 54D30

Weston, Kenneth W.

1 (with Krause, Eugene F.) On the Lie algebra of a Burnside group of exponent 5 , 27 (1971), 463-470.

20F40 20F45; 17B99 20E10

Wets, Roger J.-B.

1 (with Walkup, David W.) A Lipschitzian characterization of convex polyhedra, 23 (1969), 167-173.

$52 \mathrm{~A} 25$

Whaley, Thomas P.

1 (with Bednarek, Alexander R.) Binary relations on sets of regular cardinality, 23 (1969), 455-457.

04A05 04A10 06A05 06A10 06A20; 02F10 04A20 20M35 68A25 94A30

Whitman, David G.

1 On the multiplicity of an integral extension of a local ring, 25 (1970), 145-146.

13B20 13H15

Whittemore, Alice

1 (with Cossey, John) On the Frattini subgroup, 21 (1969), 699-702; Addendum: 27 (1971), 63-64.

20F15 20F30

Whitten, W. C., Jr.

1 On noninvertible links with invertible proper sublinks, 26 (1970), 341-346.

55A25; 55A05 57A35

Whyburn, Gordon Thomas

1 Accessibility spaces, 24 (1970), 181-185. 54B15 54D50; 54C10 54D55

Wicke, Howard H.

1 On the Hausdorff open continuous images of Hausdorff paracompact p-spaces, 22 (1969), 136-140.

54C10 54D20; 54A25 54C60

2 (with Worrell, John M., Jr.) Extension of a result of Dieudonné, 25 (1970), 634-637. 
Wickless, William J.

54D15 54D45 54E15; 54D20

1 Rings with the contraction property, 27 (1971), 57-60. 16A20 16A22 16A66 Widom, Harold

1 An inequality for rational functions, 24 (1970), 415-416.

30A04

Wiegand, Roger

1 Endomorphism rings of ideals in a commutative regular ring, 23 (1969), 442-449.

16A30; 06A40 13C05

Wilansky, Albert

1 Subalgebras of $B(X), 29$ (1971), 355-360.

Wilbur, W. John

46L20 47B05; 40H05 46E15 47A05

1 (with Pfeffer, W.) A note on cluster points of a semihereditary stable system of sets, 21 (1969), 121-125. 54A20; 28A15

2 On measurability and regularity, 21 (1969), 741-746.

02K35 28A60

Wilcox, Howard J.

1 Dense subgroups of compact groups, 28 (1971), 578-580.

$22 \mathrm{CO5} ; 54 \mathrm{H} 10$ Wilder, B. E.

1 Semigroups on acyclic plane continua, 28 (1971), 587-589.

22A15 54F20; 54H 15

Wilke, F. W.

1 (with Narayana Rao, M. L.) A necessary condition that two finite quasi-fields coordinatize isomorphic translation planes, 24 (1970), 124-125.

Wilken, Donald R.

$12 \mathrm{~K} 0550 \mathrm{D} 35$

1 Remarks on the string of beads, 23 (1969), 133-135.

$46 \mathrm{~J} 10$

2 Bounded point derivations and representing measures on $R(X), 24$ (1970), 371-373. 30A98; 46E25 47B99

\section{Willard, Stephen}

1 Metric spaces all of whose decompositions are metric, 21 (1969), 126-128.

54E35; 54C10 54C50 54D30

Williams, D. L.

1 (with Taylor, B. A.) The peak sets of $A^{m}, 24$ (1970), 604-606. 30A42 Williams, Francis D.

1 Higher homotopy commutativity and extension of maps, 26 (1970), 664-670. 55B45 55D35 55D40 55D45 55E15; 55F35 55G30

Williams, G. Kenneth

1 On continuity in two variables, 23 (1969), 580-582.

$32 \mathrm{~A} 10$

2 Continuous and proper decompositions, 28 (1971), 267-270.

Williams, James P.

1 Finite operators, 26 (1970), 129-136.

54B15 54C10; 54D05

Villiams, Kenneth S.

1 Note on a theorem of Pall, 28 (1971), 315-316. 10J05

2 Note on the Kloosterman sum, 30 (1971), 61-62. 10G05

3 Note on Salié's sum, 30 (1971), 393-394. 10G05

Williams, R. E.

1 On the free product of rings with weak algorithm, 23 (1969), 596-597. 16A06 
Williams, Richard K.

1 A note on expansive mappings, 22 (1969), 145-147.

54E40; 54C60

2 Some results on expansive mappings, 26 (1970), 655-663.

$54 \mathrm{C} 60$ 54E40; 54H20

Williams, Robert F.

1 (with Shub, Michael) Future stability is not generic, 22 (1969), 483-484.

Williams, Vernon

$58 \mathrm{~F} 10$

1 Operators from Banach spaces to complex interpolation spaces, 26 (1970), 248-254.

47A60 47A99; 46E35 47A30

Willig, Paul

$1 B(H)$ is very noncommutative, 24 (1970), 204-205. 46L10

2 Properties $\Gamma$ and $L$ for type $I I_{1}$ factors, 25 (1970), 836-837. 46L10

3 Property $P$ and direct integral decomposition of $W^{-*}$ algebras, 29 (1971), 494-498.

$46 \mathrm{~L} 10$

4 Property $L$ and direct integral decompositions of $W-*$ algebras, 30 (1971), 87-91.

Willmott, R. C.

$46 \mathrm{~L} 10$

1 On the uniformization of Souslin Fr sets, 22 (1969), 148-155.

Wils, W. I. M.

54H05; 04A15 28A05

1 On semigroups near the identity, 21 (1969), 762-763.

47D05

Wilson, M. Wayne

1 Nonnegative expansions of polynomials, 24 (1970), 100-102.

42A52 42A60; 33A65

Wilson, R. G.

1 (with De Marco, Giuseppe) Realcompactness and partitions of unity, 30 (1971), 189-194.

54C40 54D60; 54A25 54D20 54E15

Wilson, Robert Lee

1 Irreducible Lie algebras of infinite type, 29 (1971), 243-249.

17B05 17B10 17B20; 17B65 22E65 58H05

Winter, David L.

1 Finite linear groups containing an irreducible solvable normal subgroup, 25 (1970), 716.

$20 \mathrm{C} 15$

Winthrop, Joel

1 (with Cutler, Doyle O.) A note on a paper of Paul Hill and Charles Megibben, 22 (1969), 428-429.

$20 \mathrm{~K} 10$

Wiscamb, Margaret Reames

1 The discrete countable chain condition, 23 (1969), 608-612. Wishart, E.

1 (with Davis, R. D.) Galois extensions and the ramification sequence of some wildly ramified $\pi$-adic fields, 30 (1971), 212-216.

$12 \mathrm{~B} 1012 \mathrm{~B} 15$

Witthoft, William G.

1 A class of flexible nilstable algebras, 22 (1969), 361-363.

17A05 17A10 17A15 17A20 17A30; 17C05

2 (with Kass, Seymour) Irreducible polynomial identities in anticommutative algebras, 26 (1970), 1-9.

17A05 17A30; 17A10 


\section{Wogen, Warren}

1 On special generators for properly infinite von Neumann algebras, 28 (1971), 107 113.

47C10; 46L 10

Wolf, Joseph A.

1 A commutativity criterion for closed subgroups of compact Lie groups, 27 (1971), 619-622.

$22 \mathrm{CO5}$

2 Remark on discrete subgroups, 29 (1971), 423-425. 22E40

3 Remark on Siegel domains of type III, 30 (1971), 487-491. 32M15 Wolfe, Peter

1 An existence theorem for the reduced wave equation, 21 (1969), 663-666.

2 On the inverse of an integral operator, 25 (1970), 443-448. Wong, $\mathbf{C} . \mathbf{K}$.

1 On the solutions of a sequence of Lamé differential equations, 28 (1971), 481-488.

2 A uniformization theorem for arbitrary Riemann surfaces with signature, 28 (1971), 489-495.

30A46 30A58

Wong, James C. S.

1 Topological invariant means on locally compact groups and fixed points, 27 (1971), 572-578.

22D99

\section{Wong, Pak-ken}

1 (with Tomiuk, Bohdan J.) The Arens product and duality in $B^{*}$-algebras, 25 (1970), 529-535.

2 The Arens product and duality in $B^{*}$-algebras. II, 27 (1971), 535-538. 46L05

3 On the Arens product and annihilator algebras, 30 (1971), 79-83.

Wong, Pui-kei

1 A criterion for disfocality, 30 (1971), 112-114. 34A20 34C10

Wong, Raymond Y. T.

1 Some remarks on hyperspaces, 21 (1969), 600-602. 54E35

2 Extending homeomorphisms in compactification of Fréchet spaces, 25 (1970), 548 550.

3 A note on stable homeomorphisms of infinite-dimensional manifolds, 28 (1971), 271-272.

$37 \mathrm{~A} 20$ 57A20; 54H 15

Wong, Yim-ming

1 Lattice-invariant properties of topological spaces, 26 (1970), 206-208.

54D05 54D10 54D15 54D20 54D45; 06A20

Wood, Bruce

1 On $l$ - $l$ summability, 25 (1970), 433-436.

$40 \mathrm{~J} 05$

Wood, Geoffrey $\mathbf{V}$.

1 A note on isomorphisms of group algebras, 25 (1970), 771-775.

Wood, John W.

1 Klein bottles in circle bundles, 28 (1971), 607-608.

Woods, S. M. 
1 On perfect group rings, 27 (1971), 49-52.

16A26 16A48; 20C05 Worrell, John M., Jr.

1 (with Wicke, Howard H.) Extension of a result of Dieudonné, 25 (1970), 634-637. 54D15 54D45 54E15; 54D20

Wright, D. J.

1 (with Merkes, E. P.) On the univalence of a certain integral, 27 (1971), 97-100.

$30 \mathrm{~A} 32$

Wright, Fred $M$.

1 (with Baker, James D.) On integration-by-parts for weighted integrals, 22 (1969), 42-52.

2 (with Kennebeck, D. R. and Klasi, M. L.) The Gronwall inequality for weighted integrals, 30 (1971), 504-510. 26A42 26A86; 34A10 34H05

Wright, Perrin

1 Covering isotopies of $M^{n-1}$ in $N^{n}, 29$ (1971), 591-598.

57A35 57A99 57E05; 55F05 57A50

Wu, Ta-sun

1 A certain type locally compact totally disconnected topological groups, 23 (1969), 613-614.

22B05

2 (with Lee, Dong Hoon) On existence of compact open normal subgroups of 0 dimensional groups, 26 (1970), 526-528.

22D05

Wulbert, Daniel E.

1 A problem of Bosák concerning the graphs of semigroups $C(X)$ for locally connected $X, 21$ (1969), 269-272.

Wyler, Oswald

1 A characterization of regularity in topology, 29 (1971), 588-590.

Yabuta, Kôzô

54A20 54D10; 18B10 54C05 54C60

1 Unicity of the extremum problems in $H^{1}\left(U^{n}\right), 28$ (1971), 181-184.

46J15; 30A78 32A99

Yamashita, Shinji

1 A remark on Neuwirth and Newman's paper: "Positive $H^{1 / 2}$ functions are constants", 23 (1969), 147; Erratum: 25 (1970), 462.

30A78

Yang, Chung-chun

1 A generalization of a theorem of P. Montel on entire functions, 26 (1970), 332-334.

30A66 30A68 30A70

Yang, Gou-sheng

1 (with Shawyer, Bruce Lockhart Robertson) On the relation between the Abel-type and Borel-type methods of summability, 26 (1970), 323-328. 40D25 40G10

Yang, Jaw-ching

1 A theorem on the semigroup of binary relations, 22 (1969), 134-135. 20M10

Yap, Leonard Y. H.

1 (with Martin, John C.) The algebra of functions with Fourier transforms in $L^{p}, 24$ (1970), 217-219.

$43 \mathrm{~A} 15$

Yasuhara, Ann

1 The solvability of the word problem for certain semigroups, 26 (1970), 645-650.

20M05; 02F05 02G05 20F10

Yeh, J.

1 Approximate evaluation of a class of Wiener integrals, 23 (1969), 513-517.

$65 \mathrm{D} 30$ 
2 Minimal coefficients in Hölder conditions in the Wiener space, 25 (1970), 385-390.

Yeh, R. Z.

1 A geometric proof of Markov ergodic theorem, 26 (1970), 335-340.

15A51 60J10; 50D05 52A25 54H25

Yen, Ti

1 On F-normalizers, 26 (1970), 49-56.

20D10

Yohe, Cleon R.

1 Commutative rings whose matrix rings are Baer rings, 22 (1969), 189-191.

Yohe, J. M.

13E99 13F05; $13 \mathrm{C} 10$

1 Monotone mapping properties of hereditarily infinite dimensional spaces, 22 (1969), 639-645.

$54 \mathrm{~F} 20$ 54F45; 54C10

Yorke, James A.

1 Noncontinuable solutions of differential-delay equations, 21 (1969), 648-652.

$34 \mathrm{~K} 05$

2 Periods of periodic solutions and the Lipschitz constant, 22 (1969), 509-512.

$34 \mathrm{C} 25$

3 (with Strauss, Aaron) Identifying perturbations which preserve asymptotic stability, 22 (1969), 513-518.

34D10

4 (with Strauss, Aaron) Linear perturbations of ordinary differential equations, 26 (1970), 255-260.

34D 10

Young, Eutiquio C.

1 (with Diaz, Joaquin B.) Uniqueness of solutions of certain boundary value problems for ultrahyperbolic equations, 29 (1971), 569-574.

35G15; 35A05 35L20

Young, Sam W.

1 The representation of chainable continua with only two bonding maps, 23 (1969), 653-654.

54F15 54F20; 54C30 54C35

Young, W. L.

1 A product space with the fixed point property, 25 (1970), 313-317.

54H25; 54B10 54F50

Zaidman, Samuel

1 Bounded solutions of some abstract differential equations, 23 (1969), 340-342.

34D20 34G05

2 Some asymptotic theorems for abstract differential equations, 25 (1970), 521-525.

Zaks, Abraham

34D05 34G05

1 (with Evyatar, Asriel) Purely transcendental subfields of $k\left(x_{1}, \ldots, x_{n}\right), 22$ (1969), 582-586.

12F20 12F99 13F99; 13B99

2 (with Evyatar, Asriel) Rings of polynomials, 25 (1970), 559-562.

Zaks, Joseph

1 On a conjecture of A. J. Hoffman, 27 (1971), 122-125.

13B25; $13 \mathrm{C} 15$

Zalcman, Lawrence

1 Addendum to 'Analytic functions and Jordan arcs', 21 (1969), 507.

30A 30; 30A60

Zame, Alan

1 A note on Wallman spaces, 22 (1969), 141-144.

54E45; 54B99 54D35 
Zee, Yun-cheng

1 The Jacobi sums of order twenty-two, 28 (1971), 25-31.

$10 \mathrm{G} 0512 \mathrm{C} 20$

Zeitlin, David

1 A new class of generating functions for hypergeometric polynomials, 25 (1970), 405-412.

$33 \mathrm{~A} 65 ; 33 \mathrm{~A} 70$

Zelmanowitz, Julius Martin

1 (with Ware, Roger) The Jacobson radical of the endomorphism ring of a projective module, 26 (1970), 15-20. 16A21 16A42; 16A50

Zemmer, Joseph L., Jr.

1 (with Narayana Rao, M. L.) A question of Foulser on $\lambda$-systems of characteristic two, 21 (1969), 373-378.

$12 \mathrm{~K} 05 ; 50 \mathrm{D} 35$

Zenor, Phillip

1 A note on Z-mappings and WZ-mappings, 23 (1969), 273-275.

54C10 54D15; 54C50 54D30

2 A class of countably paracompact spaces, 24 (1970), 258-262. 54D20; 54A25

3 On the completeness of the space of compact subsets, 26 (1970), 190-192.

54B99 54D30 54D60

4 Countable paracompactness in product spaces, 30 (1971), 199-201.

54B10 54D15 54D20

Zettl, Anton

1 A note on square integrable solutions of linear differential equations, 21 (1969), 671-672.

34B05 34D05

2 Square integrable solutions of $L y=f(t, y), 26$ (1970), 635-639.

34B05 34D05; 47E05

3 Factorization of differential operators, 27 (1971), 425-426. 34A05

Zink, Robert E.

1 A continuous basis for Orlicz spaces, 21 (1969), 520-522. 46B15 46E30

2 On a theorem of Goffman concerning Schauder series, 21 (1969), 523-529.

$42 \mathrm{~A} 56$

Zippin, $\mathbf{M}$.

1 On some subspaces of Banach spaces whose duals are $L_{1}$ spaces, 23 (1969), 378-385.

$46 \mathrm{E} 30$

2 Existence of universal members in certain families of bases of Banach spaces, 26 (1970), 294-300.

46B15

Zlotkiewicz, Eligiusz

1 (with Reade, Maxwell O.) On univalent functions with two preassigned values, 30 (1971), 539-544.

$30 \mathrm{~A} 32$

Zuckerman, Martin M.

1 Choices from finite sets and choices of finite subsets, 27 (1971), 133-138.

02K20; 04A20 04A25 


\section{SUBJECT INDEX}

\section{0-XX GENERAL}

00-00 Difficult to classify at the second level (must also be assigned at least one other classification number in this section)

00-01 Elementary exposition (collegiate level)

00-02 Advanced exposition (research surveys, etc.)

00A05 General mathematics

00A10 Collections of papers:

Proceedings of conferences of general interest, translation volumes, etc. [See also 01A75.]

00A15 General bibliographies

00A20 Dictionaries and other general reference works

00A25 Methodology and philosophy of mathematics

\section{1-XX HISTORY AND BIOGRAPHY}

01-00 Difficult to classify at the second level (must also be assigned at least one other classification number in this section)

$01 \mathrm{~A} 05$ General histories, source books

01A10 Primitive

01A15 Pre-Greek, Babylonian, Egyptian

01A20 Greek

$01 \mathrm{~A} 25$ Far East

01A30 Islam

01A35 Medieval

$01 \mathrm{~A} 4015$ th and 16 th centuries,

Renaissance

01 A45 17th century

01 A50 18th century

01A55 19th century

01A60 20th century

01A65 Contemporary

01A70 Biographies, obituaries, personalia

01A75 Collected or selected works; reprintings or translations of classics

\section{2-XX LOGIC AND FOUNDATIONS}

02-00 Difficult to classify at the second level (must also be assigned at least one other classification number in this section)

02-01 Elementary exposition (collegiate level)

02-02 Advanced exposition (research surveys, etc.)

$02-03$ Historical

02-04 Explicit machine computation and programs (not the theory of computation or programming)

02A05 Philosophical and critical

02Bxx Classical logical systems

02B05 Propositional calculus

02B10 Predicate calculus

Monk, J. Donald

IP

02B15 Higher order predicate calculus

02B20 Unusual quantifiers

02B25 Infinitely long sentences

Eklof, Paul C.

$1 \mathrm{P}$

02B99 None of the above, but in this section

02Cxx Nonclassical formal systems

02C05 Many-valued logic

Ulrich, Dolph

Eklof, Paul C.

$1 \mathrm{P}$
$1 \mathrm{P}$

02C10 Modal logic, etc.

$02 \mathrm{C} 15$ Formalizations of intuitionism, etc.

02C20 Combinatory logic

Hindley, Roger

Lercher, Bruce

1P

$02 C 99$ None of the above, but in this section

02Dxx Proof theory \{For theorem proving by machines, see 68A40.

02D05 Proof-theoretic ordinals

02D99 Other proof theory

\section{Exx Constructive mathematics}

02E05 Intuitionistic mathematics

02E10 Algorithms

02E15 Computable functions

Aberth, Oliver

Aberth, Oliver 
02E99 None of the above, but in this section

Adler, Andrew

$1 \mathbf{P}$

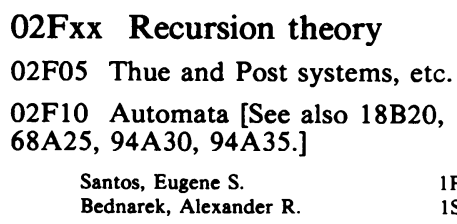

02F15 Turing machines

02F20 Classification of recursive functions

$$
\text { Payne, T. H. }
$$

02F25 Recursively enumerable sets

Robinson, Julia

02F27 Recursion theory on ordinals and sets and other abstract structures

$$
\text { Kent, C. F. }
$$

Fabian, R. J.

02F29 Recursion theory at higher types 02F30 Degrees of unsolvability

Soare, Robert I.

Platek, Richard A.

$1 \mathrm{P}$

02F35 Hierarchies

Platek, Richard A.

$1 \mathrm{P}$

02F40 Recursive equivalence types

02F43 Formal systems for computability

02F45 Combinatorial functions

$02 \mathrm{~F} 47$ Word problems

02F50 Applications

02F99 None of the above, but in this section

\section{Gxx Methodology of deductive systems}

02G05 Decidability and undecidability

$$
\text { Adler, Andrew }
$$

$1 \mathrm{P}$

02G10 Axiomatizability

$$
\text { Jones, John P. }
$$

02G15 Finite axiomatizability

02G20 Completeness, categoricity, etc.

02G99 None of the above, but in this section

\section{Hxx Model theory}

02H05 Models for theories in classical predicate calculus

02H10 Models for other theories Eklof, Paul C.

02H13 Model constructions

Adler, Andrew

02H15 Applications to algebra, number theory, etc. [See also 13L05.]

Billis, M. J.

02H20 Nonstandard models

Phillips, R. G.

$1 \mathbf{P}$

02H25 Applications of nonstandard models [See also 10N15, 12L15, 26A98, 30A91.]

02H99 None of the above, but in this section

\section{Jxx Algebraic logic}

02J05 Boolean algebras, lattices, topologies

Stringall, Robert $w$.

$2 \mathrm{~S}$

02J10 Algebra of relations

02J15 Cylindric and polyadic algebras

02J99 None of the above, but in this section

\section{Kxx Set theory}

02K05 Consistency and independence results

02K10 Nonclassical set theories

02K15 Axiomatics

02K20 Axiom of choice and equivalent propositions (Zorn's Lemma, etc.) [See also 04A25.]

$$
\begin{aligned}
& \text { Brown, Arlen } \\
& \text { Halpern, James D } \\
& \text { Howard, Paul E. }
\end{aligned}
$$

$1 \mathrm{P}$
$1 \mathrm{P}$
$1 \mathrm{P}$

$02 \mathrm{~K} 25$ Continuum hypothesis, generalized continuum hypothesis [See also 04 A 30.]

Hindman, Neil

1S

Darst, Richard B.

$5 P$

02K30 Descriptive set theory; Borel classifications, Suslin schemes, etc. [See also $04 \mathrm{~A} 15,28 \mathrm{~A} 05,54 \mathrm{H} 05$.

Darst, Richard B.

Mansfield, Richard

Darst, Richard B.

Hansell, Roger W.

Darst, Richard B.

$3 P$
$1 P$
$4 P$
$1 P$
$5 P$

02K35 Large cardinals and ordinals 
(inaccessible, etc.)

Jorgensen, Murray

Adler, Andrew

Kleinberg, E. M.

also $02 \mathrm{~K} 25$.]

Hindman, Neil

Darst, Richard B.

$5 P$

05-XX COMBINATORICS

\{For finite fields, see $12 \mathrm{Cxx}$.

05-00 Difficult to classify at the second level (must also be assigned at least one other classification number in this section)

05-01 Elementary exposition (collegiate level)

05-02 Advanced exposition (research surveys, etc.)

05-03 Historical

05-04 Explicit machine computation and programs (not the theory of computation or programming)

\section{Axx Classical combinatorial problems}

05A05 Combinatorial choice problems; subsets, representatives

Morse, Anthony P.

Sharp, Henry S.

04A10 Transfinite numbers

Bednarek, Alexander R.

Jorgensen, Murray

Adler, Andrew

04A15 Descriptive set theory; Borel classifications, Suslin schemes, etc. [See also $02 \mathrm{~K} 30,28 \mathrm{~A} 05,54 \mathrm{H} 05$.]

Willmott, R. C.
Stone, A. H.
Erdös, Paul
Mrówka, Stanislaw
Darst, Richard B.
Sharp, Henry S.
Mansfield, Richard
Darst, Richard B.
Hansell, Roger W.
Darst, Richard B.

04A20 Combinatorial [See also 05A05.]

Walker, Elbert A

Richman, Fred

Brown, Terrence J.

Bednarek, Alexander R.

Kleinberg, E. M.

04A25 Axiom of choice and equivalent propositions (Zorn's Lemma, etc.) [See also 02K20.]

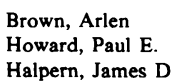

04A30 Continuum hypothesis, generalized continuum hypothesis [See

$\begin{array}{ll}\text { Brown, Terrence J. } & 1 \mathrm{P} \\ \text { Kohn, S. } & 1 \mathrm{~S} \\ \text { Chvátal, Václav } & 1 \mathrm{P} \\ \text { Kleitman, Daniel J. } & 3 \mathrm{P} \\ \text { Erdös, Paul } & 2 \mathrm{P}\end{array}$

Kleitman, Daniel J.
Erdös, Paul

05A10 Factorials, binomial coefficients, combinatorial functions

Sharp, Henry S.

Howard, F. T.

$3 P$

05A15 Combinatorial enumeration problems, generating functions

Vidyasagar, $\mathbf{M}$.

Subbarao, M. V.

Howard, F. T

05A17 Partitions [See also 10A45.]

05A19 Combinatorial identities

Vidyasagar, $\mathrm{M}$.

Subbarao, M. V.

05A20 Combinatorial inequalities

$\begin{array}{ll}\text { Erdös. Paul } & 2 \mathrm{P} \\ \text { Kleitman, Daniel J. } & 3 \mathrm{P}\end{array}$

05A99 None of the above, but in this section

05Bxx Designs and configurations

05B05 Block designs [See also 62Kxx.]

05B10 Difference sets (number-theoretic, group-theoretic, etc.)

Payne, Stanley E.

$1 \mathrm{P}$ 
05B15 Orthogonal arrays, Latin squares 05B20 Matrices (incidence, Hadamard, etc.)

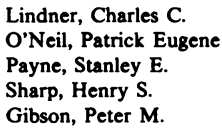

05B25 Finite geometries

Johnson, Norman Lloyd Mason, J. H.

05B30 Other designs, configurations

Kleitman, Daniel J.

Amoroso, $\mathrm{S}$.

Cooper, G.

Lindner, Charles C.

05B35 Matroids, geometric lattices

Mason, J. H. $1 P$

05B40 Packing and covering [See also 10E30, 52A45.]

05B45 Tessellation and tiling problems 05B99 None of the above, but in this section

05Cxx Graph theory \{For applications of graphs, see 94A20. 05C05 Trees

05C10 Topological graph theory, embedding [See also 55A 15, 55A25.]

Marx, Morris L.

05C15 Chromatic theory of graphs and maps

Chvátal, Václav 1P

05C20 Directed graphs (digraphs)

Plemmons, Robert J.

$1 \mathrm{P}$

05C25 Graphs and groups

Plemmons, Robert J.

Lin, You-feng

1P

05C30 Enumeration of graphs and maps

Swan, Richard G.

O'Neil, Patrick Eugene

Chvátal, Václav

1P

$1 \mathrm{P}$

05C35 Paths and extremal problems

O'Neil, Patrick Eugene

2P

05C99 None of the above, but in this section

Lin, You-feng

Ratti, Jogindar S

Rosenfeld, M.

Behzad, Mehdi

Schwartz, Benjamin L.
Beineke, Lowell W.

Greenwell, D. L.

1P
IP

\section{6-XX ORDER, LATTICES,} ORDERED ALGEBRAIC STRUCTURES

06-00 Difficult to classify at the second level (must also be assigned at least one other classification number in this section)

06-01 Elementary exposition (collegiate level)

06-02 Advanced exposition (research surveys, etc.)

06-03 Historical

06-04 Explicit machine computation and programs (not the theory of computation or programming)

06A05 Total order

Cobb, John

Bednarek, Alexander R.

Armacost, David Lee

06A10 Partial order

DeMarr, Ralph E.

Bednarek, Alexander $\mathbf{R}$

Schelp, R. H.

Gudder, Stanley P.

Davies, Roy $O$.

Rousseau, George

Hayes, Allan

Venkatanarasimhan, P. V.

Smithson, Raymond E.

Gudder, Stanley P.

Haskins, L.

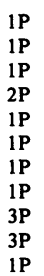

06A15 Galois correspondence, closure operators

06A20 Lattices, semi-lattices, generalizations

Evans, Trevor
Dean, Richard A.
Bednarek, Alexander R.
Hales, A. W.
Wong, Yim-ming
Rousseau, George
Davies, Roy O.
Hayes, Allan
Venkatanarasimhan, P. V.
Stepp, James W.
Hursey, R. J., Jr.
To, Ting On

06A23 Complete lattices

Jensen, G. A

Randall, Charles $\mathbf{H}$.

Holland, Samuel S., Jr.

Olson, Milton Philip

Catlin, Donald E.

06A25 Complemented lattices, 
generalizations

Randall, Charles H.
Anderson, Bruce A.
Stewart, D. G.
Holland, Samuel S., Jr.
Schelp, R. H.
Gudder, Stanley P.
Catlin, Donald E.

1P
1S
1S
1P
1S
$2 S$
1P

06A30 Modular lattices, continuous geometries, generalizations [See also 16A30.]

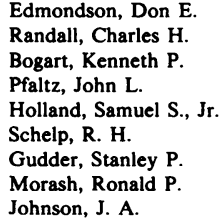

06A35 Distributive lattices, generalizations

Choe, Tae Ho

Lakser, $\mathrm{H}$.

Balbes, Raymond

Catlin, Donald E.

06A40 Boolean algebras and rings

Bogart, Kenneth P.
Wiegand, Roger
Abian, Alexander
Lakser, H.
Holland, Samuel S., Jr.
Chew, Kim-peu
Venkatanarasimhan, P. V.
Stringall, Robert W.
Nosal, Miloslav
Stringall, Robert W.
Catlin, Donald E.

06A45 Order topologies [See also 54F05.]

Lutzer, David J.
Bennett, Harold R.
Lutzer, David J.
Gulden, Samuel L.
Weston, J. H.
Fleischman, W. M.
Madell, Robert L.
Madell, Robert L.
Breuer, Shlomo

Breuer, Shlomo

06A50 Ordered semigroups, other generalizations of groups

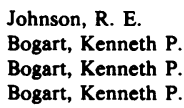

06A55 Ordered groups

$$
\begin{aligned}
& \text { Madell, Robert L. } \\
& \text { Hursey, R. J., Jr. }
\end{aligned}
$$$$
\text { Minassian, Donald P }
$$

06A60 Ordered abelian groups

$\begin{array}{ll}\text { Kohls, Carl W. } & \text { 1P } \\ \text { Chambless, Donald A. } & \text { 1P } \\ \text { Minassian, Donald P. } & \text { 1S }\end{array}$

06A65 Ordered linear spaces [See also 46A40.]

Waterman, Alan G.

Chambless, Donald A.

IP

06A70 Ordered rings, algebras, modules [For ordered fields, see $10 \mathrm{M} 15,12 \mathrm{~J} 15$.]

$\begin{array}{ll}\text { DeMarr, Ralph E. } & \text { 1P } \\ \text { Abian, Alexander } & \text { IS } \\ \text { Kohls, Carl W. } & \text { IP } \\ \text { Carns, Gail L. } & \text { 1P } \\ \text { Gellar, Ralph } & \text { 2P }\end{array}$

06A75 Other ordered algebraic structures

\section{8-XX GENERAL MATHEMATICAL SYSTEMS}

08-00 Difficult to classify at the second level (must also be assigned at least one other classification number in this section)

08-01 Elementary exposition (collegiate level)

08-02 Advanced exposition (research surveys, etc.)

08-03 Historical

08-04 Explicit machine computation and programs (not the theory of computation or programming)

08A05 Structure of general algebras and relational systems [For topological representations, see $54 \mathrm{H} 10$.]

$\begin{array}{ll}\text { Horn, Alfred } & 1 \mathrm{P} \\ \text { Padmanabhan, R. } & 1 \mathrm{P} \\ \text { Grätzer, György } & 1 \mathrm{P}\end{array}$

08A10 Free algebras, sums, products, limits, colimits

Hales, A. W.
Davis, Robert
Huq, Syed A.
Balbes, Raymond

08A15 Varieties of algebras and generalizations

$$
\begin{aligned}
& \text { Evans, Trevor } \\
& \text { Dean, Richard A. } \\
& \text { Le Tourneau, J. J. } \\
& \text { Comer, Stephen D. } \\
& \text { Burris, S. }
\end{aligned}
$$

08A20 Axiomatic model classes 08A25 Universal algebra 
Grätzer, György

10-XX NUMBER THEORY \{Excluding 10Axx and 10Mxx, this classification scheme does not distinguish work in the rational number fields from that in other algebraic number fields. \}

10-00 Difficult to classify at the second level (must also be assigned at least one other classification number in this section)

10-01 Elementary exposition (collegiate level)

10-02 Advanced exposition (research surveys, etc.)

10-03 Historical

10-04 Explicit machine computation and programs (not the theory of computation or programming)

10Axx Elementary number theory \{For analogues in number fields, see 12A05.

10A05 Multiplicative structure of the integers (GCD, etc.)

10A10 Congruences, primitive roots

Vanden Eynden, C. L. Crittenden, Richard B.

Howard, F. T.

$1 \mathrm{P}$
$1 \mathrm{P}$
$2 \mathrm{P}$

10A15 Power residues, reciprocity Brown, Ezra

$1 \mathrm{P}$

10A20 Number-theoretic functions, related numbers; inversion formulas

Brown, Terrence J.

Buschman, R. G.

Howard, F. T.

Aizley, Paul

10A25 Elementary prime number theory, factorization

Howard, F. T

10A30 Algorithms and expansions, digital properties [Approximation results in 10F20; metric results in 10K10.]

Newman, Donald J.

Cusick, T. W.

10A35 Recurrence sequences

10A40 Special numbers, sequences and polynomials (e. g. Bernoulli)

Howard, F. T

\section{A45 Partitions}

Alder, Henry $L$.

Subbarao, M. V.

Subbarao, M. V.

1P

10A99 None of the above, but in this section

10Bxx Diophantine equations (For all papers involving algebro-geometric methods, see

$14 \mathrm{Gxx}$.

10B05 Linear, quadratic and bilinear equations

10B10 Cubic and quartic equations

Webb, William A. Cohn, John H. E.

10B15 Higher degree equations

Mordell, Louis Joel

$1 \mathrm{P}$

10B20 Multiplicative equations

10B25 Nonpolynomial equations

$10 B 30$ Equations in sufficiently many variables [See also 10J10.]

10B35 Representation problems [See also 10J10.]

Cohn, John H. E. $1 \mathbf{P}$

$10 \mathrm{~B} 40 \mathrm{p}$-adic and power series fields

10B45 Diophantine inequalities

10B99 None of the above, but in this section

\section{Cxx Forms}

$10 \mathrm{C} 05$ Quadratic and hermitian forms [See also 10E25.]

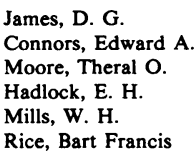

Rice, Bart Francis

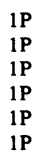

10C10 Higher degree forms

$10 \mathrm{C} 15$ Analytic theory

$10 \mathrm{C} 25$ Minima of forms [See also 10E20.]

10C30 Arithmetic properties of classical groups [See also 20Gxx.]

$10 \mathrm{C} 99$ None of the above, but in this section

10Dxx Automorphic theory

10D05 Modular functions and groups 
Hall, Michael $\mathrm{H}$

$1 \mathrm{P}$

10D10 Automorphic functions, one variable

10D15 Automorphic forms, one variable

10D20 Automorphic theory, several variables

10D25 Complex multiplication [See also 14K22.]

10D99 None of the above, but in this section

\section{Exx Geometry of numbers}

10E05 Lattices and convex bodies [See also 10J25.]

$$
\text { Wang, S. P. }
$$

10E10 Nonconvex bodies

$$
\text { Priestley, W. M. }
$$

10E15 Products of linear forms

10E20 Minima of forms

$$
\text { Cooke, Roger }
$$

10E25 Quadratic forms (reduction, extreme forms, etc.)

10E30 Lattice packing and covering [See also 05B40, 52A40.]

10E35 Mean value theorems

10E40 Transfer theorems

$10 \mathrm{E} 45$ Automorphism groups of lattices

10 E99 None of the above, but in this section

10Fxx Diophantine approximation \{See also 10Kxx.\}

10F05 Approximation to one number

10F10 Simultaneous approximation

10F15 Nonhomogeneous approximation

10F20 Continued fractions and generalizations [See also 10A30.]

$\begin{array}{ll}\text { Bernstein, Leon } & \text { 1P } \\ \text { Lee, R. A. } & \text { 1P } \\ \text { Cusick, T. W. } & \text { 2P }\end{array}$

10F25 Approximation to algebraic numbers

10F30 Approximation by numbers from a fixed field

10F35 Irrationality and transcendence

10F40 Distribution modulo one [See also 10K05.]

Boyd, David W.

$1 \mathbf{P}$

O'Neil, Patrick Eugene

$1 \mathrm{P}$

10F45 Approximation in nonarchimedean valuations

10F99 None of the above, but in this section

10G05 Exponential sums, character sums

$$
\begin{aligned}
& \text { Apostol, Tom M. } \\
& \text { Zee, Yun-cheng } \\
& \text { Williams, Kenneth S. } \\
& \text { Williams, Kenneth S. }
\end{aligned}
$$

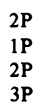

$10 \mathrm{Hxx}$ Multiplicative theory

10H05 Riemann zeta-function

Spira, Robert

$1 \mathrm{P}$

$10 \mathrm{H} 10$ Other zeta-functions, L-functions

[For local and global ground fields, see

$12 \mathrm{~A} 70,12 \mathrm{~A} 80,12 \mathrm{~B} 30,12 \mathrm{~B} 35$; for

algebro-geometric methods, see 14G10.]

Berndt, Bruce $\mathrm{C}$.

2P

$10 \mathrm{H} 15$ Distribution of primes and of integers with specified multiplicative properties

Suryanarayana, D.

Sitaramachandra Rao, $\mathbf{R}$.

$1 \mathrm{P}$

$10 \mathrm{H} 20$ Distribution in progressions and other sequences

$10 \mathrm{H} 25$ Asymptotic results on arithmetic functions

$$
\begin{aligned}
& \text { Niven, Ivan } \\
& \text { Uchiyama, Saburô }
\end{aligned}
$$

$10 \mathrm{H} 30$ Sieves

10H35 Distribution of residue classes (primitive roots, power residues, etc.)

$10 \mathrm{H} 40$ Generalized primes and integers

10 H99 None of the above, but in this section

\section{Jxx Additive theory}

$10 J 05$ Sums of squares

$$
\text { Williams, Kenneth S. }
$$

10J10 Applications of Hardy-Littlewood method [See also 10B35.]

Knopfmacher, J.

Sinclair, Allan M.

$10 \mathrm{~J} 15$ Additive questions involving primes

$10 J 20$ Analytic work on partitions [See also 10A45.]

Andrews, George E.

2P 
$10 \mathrm{~J} 25$ Lattice points in large regions $10 J 99$ None of the above, but in this section

10Kxx Probabilistic theory;
measure, dimension, etc.
10K05 Distribution modulo one
10K10 Algorithms and expansions
10K15 Diophantine approximation
Liu, Ming-chit
10K20 Arithmetic functions
Duncan, R. L.

$10 \mathrm{~K} 25$ Normal numbers

$10 \mathrm{~K} 30$ Irregularities of distribution

10K35 Harmonic analysis and almost periodicity

10K40 Nonarchimedean theory

10K99 None of the above, but in this section

\section{Lxx Sequences of integers}

10L05 Addition of sequences

10L10 Special sequences (density, etc.)

10L15 Representation functions

10L99 None of the above, but in this section

10Mxx Rational arithmetic of algebraic objects

10M05 Polynomials [See also 13F20.]

Enochs, Edgar E.

Coleman, Donald B.

$10 \mathrm{M} 10$ Valued fields

$10 \mathrm{M} 15$ Ordered fields

10M20 Matrices [See also 15A36.]

10M99 None of the above, but in this section

\section{Nxx Connections with logic} 10N05 Decidability

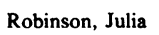

2P

10 N10 Ultraproducts

10 N15 Nonstandard arithmetic [See also 02H25.]

10N99 None of the above, but in this section

\section{2-XX ALGEBRAIC}

\section{NUMBER THEORY, FIELD THEORY AND \\ POLYNOMIALS}

12-00 Difficult to classify at the second level (must also be assigned at least one other classification number in this section)

12-01 Elementary exposition (collegiate level)

12-02 Advanced exposition (research surveys, etc.)

12-03 Historical

12-04 Explicit machine computation and programs (not the theory of computation or programming)

12Axx Algebraic number theory: global fields (For complex multiplication, see 10D25.

12A05 Analogues in number fields of elementary number theory

12 A 10 Characterizations of algebraic numbers and algebraic functions

$12 \mathrm{~A} 15$ Special algebraic numbers (PV numbers, etc.)

12A20 Polynomials (irreducibility, etc.)

12A25 Quadratic fields

$$
\begin{aligned}
& \text { Stark, H. M. } \\
& \text { Trotter, Hale F. }
\end{aligned}
$$

Hall, Michael $\mathrm{H}$.

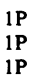

12A30 Cubic and quartic fields

12A35 Abelian and metabelian extensions (including cyclotomic, Kummer, cyclic)

Smith, John $\mathrm{H}$.

$12 \mathrm{~A} 40$ Other number fields

12A45 Units and factorization

Trotter, Hale F. May, Warren L.

12 A50 Class number, discriminant

Stark, H. M

$1 \mathrm{P}$

12A55 Galois theory

$12 \mathrm{~A} 60$ Galois cohomology

$12 \mathrm{~A} 65$ Class field theory

Brown, Ezra

12 A70 Zeta-functions and L-functions of number fields [See also 10H10.] 
12 A75 Density theorems

12 A80 Arithmetic and zeta-functions of algebras

12 A85 Analysis in adèle rings and groups

12A90 Arithmetic theory of algebraic function fields [See also 14-XX.]

12A95 Totally real and totally positive fields

12A99 None of the above, but in this section

12Bxx Algebraic number theory: local and p-adic fields

12B05 Polynomials

Hilliker, D. L.

$1 \mathrm{P}$

12B10 Ramification and extension theory

Wishart, E.

1P

12B15 Galois theory

Wishart, E.

12B20 Galois cohomology

12B25 Class field theory

12 B30 Zeta- and L-functions [See also 10H10.]

12 B35 Arithmetic and zeta-functions of algebras

$12 \mathrm{~B} 40$ Other analytic theory

12 B99 None of the above, but in this section

12Cxx Finite fields and finite commutative rings

12C05 Polynomials

Niederreiter, Harald

$1 \mathrm{P}$

$12 \mathrm{C} 10$ Linear sequences

$12 \mathrm{C} 15$ Arithmetic

12 C20 Cyclotomy

Zee, Yun-cheng

$1 P$

$12 \mathrm{C} 25$ Exponential sums

Niederreiter, Harald

$12 \mathrm{C} 30$ Structure theory

Tillman, Stephen J.

$12 C 99$ None of the above, but in this section

12Dxx Real and complex fields
12D05 Polynomials: factorization

12D10 Polynomials: location of zeros

[Algebraic theorems; for the analytic

theory, see 26A78, 30A08.]

Peyser, Gideon
Marden, Morris
Flaschka, Hermann

12D15 Formally real fields

Schwabhäuser, Wolfram

12D99 None of the above, but in this section

Haeuslein, G. K.

$1 \mathbf{P}$

12Exx General field theory

12E05 Polynomials (irreducibility, etc.)

12E10 Special polynomials

Apostol, Tom $\mathrm{M}$. $1 \mathrm{P}$

12E99 None of the above, but in this section

Kohn, S.

$1 \mathbf{P}$

12Fxx Field extensions

12 F05 Algebraic extensions

12 F10 Separable extensions, Galois theory

$\begin{array}{ll}\text { Perlis, Sam } & 1 S \\ \text { Isaacs, I. M. } & 2 P \\ \text { Accola, Robert D. M. } & \text { 3S } \\ \text { Greenleaf, Newcomb } & \text { 2P }\end{array}$

12 F 15 Inseparable extensions, derivations

Vinograde, Bernard Mordeson, John N

12F20 Transcendental extensions

Zaks, Abraham

12 F99 None of the above, but in this section

Zaks, Abraham

$1 \mathrm{P}$

12Gxx Homological methods

12G05 Galois cohomology [See also 12A60, 18H10.]

Leitzel, J. R. C $1 \mathrm{P}$

12G10 Cohomological dimension

12G99 None of the above, but in this section

$12 \mathrm{Hxx}$ Differential and difference algebra

12H05 Differential algebra 
Seidenberg, A

Blum, Peter

Goldman, Lawrence

Newton, M. E.

12H10 Difference algebra

Franke, Charles $\mathrm{H}$.

12Jxx Topological fields

12J05 Normed fields

$12 \mathrm{~J} 10$ Valued fields

$12 \mathrm{~J} 15$ Ordered fields

Lachlan, A. H.

Madison, Eugene W.

Carns, Gail L.

12 J20 General valuation theory

Bergman, George M.

12J99 None of the above, but in this section

Kiltinen, John $\mathrm{O}$.

12K05 Near-fields and other generalizations

$\begin{array}{ll}\text { Narayana Rao, M. L. } & \text { IP } \\ \text { Wilke, F. W. } & \text { IP } \\ \text { Narayana Rao, M. L. } & \text { P } \\ \text { Narayana Rao, M. L. } & \text { 3S } \\ \text { Narayana Rao, M. L. } & \text { 4S }\end{array}$

12Lxx Connections with logic

12L05 Decidability

12L10 Ultraproducts

12L15 Nonstandard arithmetic [See also 02H25.]

12 L99 None of the above, but in this section

Lachlan, A. H.

Madison, Eugene W.

$1 \mathrm{P}$
$1 \mathrm{P}$

13-XX COMMUTATIVE RINGS AND ALGEBRAS For finite commutative rings, see

$12 \mathrm{Cxx}$.

13-00 Difficult to classify at the second level (must also be assigned at least one other classification number in this section)

13-01 Elementary exposition (collegiate level)

13-02 Advanced exposition (research surveys, etc.)

13-03 Historical
13-04 Explicit machine computation and programs (not the theory of computation or programming)

13Axx General commutative ring theory

13A05 Divisibility

$$
\text { Canfell, M. J. }
$$

$1 \mathbf{P}$

13A10 Radical theory

$\begin{array}{ll}\text { Abian, Alexander } & \text { 1S } \\ \text { Orsatti, Adalberto } & \text { 1P } \\ \text { De Marco, Giuseppe } & \text { 2P }\end{array}$

13A15 Ideals, prime ideals, valuations and generalizations

$\begin{array}{ll}\text { Heinzer, William J. } & \text { 1P } \\ \text { Warfield, R. B., Jr. } & 3 \mathrm{~S} \\ \text { Fields, David E. } & 1 \mathrm{P} \\ \text { Bergman, George M. } & 1 \mathrm{P} \\ \text { Johnson, E. W. } & 1 \mathrm{~S} \\ \text { Lediaev, John P. } & 1 \mathrm{~S} \\ \text { Heinzer, William J. } & 2 \mathrm{P} \\ \text { Warner, Seth } & 2 \mathrm{P} \\ \text { McCarthy, Paul J. } & 1 \mathrm{P} \\ \text { Kelly, Patrick H. } & 1 \mathrm{P} \\ \text { Larsen, Max D. } & 2 \mathrm{P} \\ \text { Smith, William W. } & 1 \mathrm{P} \\ \text { De Marco, Giuseppe } & 2 \mathrm{P} \\ \text { Orsatti, Adalberto } & 1 \mathrm{P}\end{array}$

13A20 Brauer group [See also 16A16.]

Schwartz, Alan L.
Heinzer, William J.
Auslander, Bernice

2S

2P

13A99 None of the above, but in this section

$\begin{array}{ll}\text { Abian, Alexander } & \text { 1P } \\ \text { Vasconcelos, Wolmer V. } & \text { 2S }\end{array}$

13Bxx Ring extensions and related topics

13B05 Galois theory

Franke, Charles $\mathrm{H}$.

$1 \mathbf{P}$

13B10 Automorphisms and derivations

Heinzer, Martin N.

IP

13B15 Ramification theory

Heinzer, Martin N.

$1 \mathbf{P}$

13B20 Integral dependence; integral closure; integrally closed rings, related rings (Japanese, etc.)

Mattuck, Arthur

Seidenberg, A

$2 \mathbf{P}$
$2 \mathrm{~S}$

13B25 Polynomials over commutative rings

Arnold, Jimmy $T$.

Gilmer, Robert W., Jr.

$1 \mathbf{P}$
$2 \mathbf{P}$ 


$\begin{array}{ll}\text { Evyatar, Asriel } & 2 \mathrm{P} \\ \text { Brewer, J. W. } & 1 \mathrm{P} \\ \text { Arnold, Jimmy T. } & 2 \mathrm{P} \\ \text { Seidenberg, A. } & 2 \mathrm{P} \\ \text { Lindberg, John A., Jr. } & 1 \mathrm{~S}\end{array}$

13B99 None of the above, but in this section

Zaks, Abraham

Kohls, Carl W.

Fields, David E.

13Cxx Theory of modules and ideals

13C05 Structure, classification theorems

$\begin{array}{ll}\text { Bogart, Kenneth P. } & \text { 2S } \\ \text { Wiegand, Roger } & \text { 1S } \\ \text { Warfield, R. B., Jr. } & \text { 3P } \\ \text { Vasconcelos, Wolmer V. } & \text { 2P } \\ \text { Waterhouse, William C. } & \text { 1P } \\ \text { Johnson, J. A. } & \text { 1S } \\ \text { Bang, Chang Mo } & \text { 1P } \\ \text { Shores, T. S. } & \text { 1P }\end{array}$

$13 \mathrm{C} 10$ Special types (projective, injective, free, flat, torsion, reflexive, etc.) [See also 18G05.]

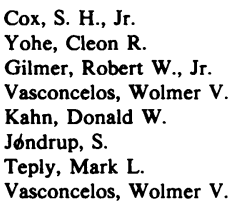

15 Dimension theory, depth, related rings (catenary, etc.)

$\begin{array}{ll}\text { Evyatar, Asriel } & 2 \mathrm{~S} \\ \text { Vasconcelos, Wolmer V. } & 2 \mathrm{P}\end{array}$

$13 C 99$ None of the above, but in this section

Vasconcelos, Wolmer V.

13Dxx Homological methods \{For cohomology of rings and algebras, see $18 \mathrm{H} 20$.

13D05 Homological dimension

Vasconcelos, Wolmer V.

3S

13D10 Deformations and infinitesimal methods

13D15 Class group, Grothendieck group, $\mathrm{K}$-theory [See also 18F25.]

Gersten, Stephen M.

1P

13D99 None of the above, but in this section
Davis, Robert $2 P$

Vasconcelos, Wolmer V.

\section{Exx Chain conditions}

\section{E05 Noetherian rings}

$\begin{array}{lr}\text { Hochster, M. } & \text { 1S } \\ \text { Heinzer, William J. } & \text { 1P } \\ \text { Fields, David E. } & \text { iS } \\ \text { Lediaev, John P. } & \text { 1S } \\ \text { Johnson, E. W. } & \text { iS }\end{array}$

13E 10 Artin rings, finite dimensional algebras

$\begin{array}{ll}\text { Dickson, Spencer E. } & \text { IP } \\ \text { Fuller, Kent R. } & \text { IP } \\ \text { Vasconcelos, Wolmer V. } & \text { 2P }\end{array}$

13E99 None of the above, but in this section

$\begin{array}{lr}\text { Yohe, Cleon R. } & 1 \mathrm{P} \\ \text { Huckaba, James A. } & 1 \mathrm{~S} \\ \text { Seidenberg, A. } & 2 \mathrm{P}\end{array}$

13Fxx Arithmetic rings

13F05 Dedekind and Prüfer rings and their generalizations

$\begin{array}{ll}\text { Yohe, Cleon R. } & \text { 1P } \\ \text { Arnold, Jimmy T. } & 2 \text { P } \\ \text { Brewer, J. W. } & \text { IP } \\ \text { Jacohinski, H. } & 2 S \\ \text { Lediaev, John P. } & \text { IP } \\ \text { Johnson, E. W. } & \text { 1P } \\ \text { McCarthy, Paul J. } & \text { IS } \\ \text { Shores, T. S. } & \text { 1S }\end{array}$

13F10 Principal ideal rings

13F15 Factorial rings, unique factorization domains

13F20 Polynomial rings [See also 10M05.]

Arnold, Jimmy T.

Gilmer, Robert W., Jr.

Coleman, Donald B.

Enochs, Edgar E.

Jøndrup, S.

13 F99 None of the above, but in this section

Zaks, Abraham

$1 \mathbf{P}$

\section{G05 Integral domains}

Gilmer, Robert W., Jr.

Beauregard, Raymond A.

Johnson, R. E.

Kohls, Carl W.

$13 \mathrm{Hxx}$ Local rings and semilocal rings

13H05 Regular local rings

Lipman, Joseph

Bogart, Kenneth P. 
Barshay, Jacob

13H10 Special types (Macaulay, Gorenstein, etc.)

$13 \mathrm{H} 15$ Multiplicity theory and related topics

13 H99 None of the above, but in this section

Ingraham, Edward C.
Brown, William C.
Lediaev, John P.
Johnson, E. W.
Riley, John A.

13J $\mathrm{Jx}$ Topological rings \{For ordered rings, see 06A70; see also 16A80.

$13 \mathrm{~J} 05$ Power series rings

Fields, David E.

$13 \mathrm{~J} 10$ Complete rings

$13 \mathrm{~J} 15$ Henselian rings

Ingraham, Edward C

Brown, William C.

$13 \mathrm{~J} 20$ Global topological rings

Evans, E. Graham, Jr section

Hochster, M

Warner, Seth

13K05 Witt vectors and related rings

13L05 Applications of logic to commutative algebra

Greenleaf, Newcomb Mott, Joe Leonard

$2 \mathrm{~S}$
is

\section{4-XX ALGEBRAIC} GEOMETRY

14-00 Difficult to classify at the second level (must also be assigned at least one other classification number in this section)

14-01 Elementary exposition (collegiate level)

14-02 Advanced exposition (research surveys, etc.)

14-03 Historical

14-04 Explicit machine computation and programs (not the theory of computation or programming)

\section{Axx Foundations}

14A05 Relevant commutative algebra [See also 13-XX.]

Mattuck, Arthur

2P

14A10 Varieties

14A15 Schemes

14A20 Generalizations (algebraic spaces, motifs)

14A25 Elementary questions

Mattuck, Arthur

Mott, Joe Leonard

2P

14A99 None of the above, but in this section

Vasconcelos, Wolmer V.

1S

14Bxx Local theory

14B05 Singularities [See also 14E15.]

Lipman, Joseph

14B10 Infinitesimal methods

14B15 Local cohomology [See also 18F20.]

14B20 Formal neighborhoods

14B99 None of the above, but in this section

14Cxx Cycles and subschemes

14C05 Parametrization (Chow and Hilbert schemes)

$14 \mathrm{C} 10$ Equivalence relations

$14 \mathrm{C} 15$ Rational equivalence rings, intersection theory

14C20 Divisors, linear systems, Picard groups

14C25 Zero-cycles

Mattuck, Arthur

$1 \mathrm{P}$

14C30 Transcendental methods, Hodge theory

14 C99 None of the above, but in this section

\section{Dxx Families, fibrations}

14D05 Structure of families (Picard-Lefschetz, Picard-Fuchs theory, etc.)

14D10 Arithmetic ground fields (finite, local, global)

14D15 Formal methods; deformations [See also 32Gxx.] 
14D20 Algebraic moduli problems [For analytic moduli problems, see $32 \mathrm{G} 13$.]

14D99 None of the above, but in this section

\section{Exx Mappings and} correspondences

14E05 Rational maps, birational correspondences

14E10 General correspondences

$14 \mathrm{E} 15$ Global theory of singularities, resolution

$$
\text { Lipman, Joseph }
$$

14E20 Coverings, fundamental group

14E25 Embeddings

14E30 Minimal models

14E35 Results in dimension $\leq 3$

14E99 None of the above, but in this section

14Fxx Cohomology theory \{See also 13Dxx, 18-XX.\}

14F05 Vector bundles, sheaves, related constructions

14F10 Differentials and other special sheaves

14F15 Serre cohomology, K-theory [See also 13D15, 18F25.]

Roberts, Leslie G.

1P

14F20 Grothendieck cohomology and topology

14F25 Classical real and complex cohomology

14F30 p-adic cohomology

14F35 Homotopy theory

14F99 None of the above, but in this section

14Gxx Arithmetic problems (For complex multiplication, see 10D25, 14K22.\}

14G05 Rationality questions

Mordell, Louis Joel

$1 \mathrm{P}$

14G10 Zeta-functions and related questions

14G13 Weil-Tate conjectures

14G15 Finite ground fields
Leitzel, J. R. C.

$2 \mathrm{P}$

$14 \mathrm{G} 20$ p-adic ground fields (local fields)

14G25 Global ground fields

14 G99 None of the above, but in this section

\section{Hxx Curves}

14H05 Algebraic function fields

14H10 Families, moduli (algebraic)

Mumford, David

14H15 Families, moduli (analytic) [See also 32G15.]

Mumford, David

1S

14H20 Singularities, local rings

$14 \mathrm{H} 25$ Arithmetic ground fields

14H30 Coverings, fundamental group

14H35 Correspondences

$14 \mathrm{H} 40$ Jacobians [See also 32G20.]

14H45 Special curves

$14 \mathrm{H} 99$ None of the above, but in this section

14Jxx Surfaces and 3-dimensional varieties

14J05 Picard group

14J10 Families, moduli, classification: algebraic theory

14J15 Singularities, moduli,

classification: analytic theory [See also 32J15.]

$$
\text { Mattuck, Arthur }
$$$$
2 \mathrm{P}
$$

14J20 Arithmetic ground fields

14J25 Special surfaces

$$
\text { Mattuck, Arthur }
$$

14J99 None of the above, but in this section

$14 \mathrm{Kxx}$ Abelian varieties and schemes

14K05 Algebraic theory

14K10 Algebraic moduli, classification

MacLachlan, C. is

14K15 Arithmetic ground fields

14K20 Analytic theory

14K22 Complex multiplication [See also 10D25.] 


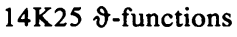

14K30 Picard schemes, higher Jacobians $14 \mathrm{~K} 99$ None of the above, but in this section

Davis, Robert

$3 \mathrm{~S}$

14Lxx Group schemes \{For linear algebraic groups, see $20 \mathrm{Gxx}$.

14L05 Formal groups, p-divisible groups

14 L10 Group varieties

Grosshans, Frank

$1 \mathrm{P}$

14 L15 Group schemes

14L20 Finite group schemes

Shatz, Stephen S.

Efroymson, Gustave

$1 \mathrm{P}$

14L25 Pro-algebraic group schemes

14L99 None of the above, but in this section

\section{Mxx Special varieties}

14M05 Varieties defined by ring conditions (factorial, Macaulay)

14M10 Complete intersections

14M15 Grassmannians, homogeneous spaces

14M20 Rational varieties

14M99 None of the above, but in this section

\section{Nxx Classical methods and} problems

14N05 Projective techniques

14N10 Enumerative problems

14N99 None of the above, but in this section

\section{5-XX LINEAR AND} MULTILINEAR ALGEBRA; MATRIX THEORY (finite and infinite)

15-00 Difficult to classify at the second level (must also be assigned at least one other classification number in this section)

15-01 Elementary exposition (collegiate level)

15-02 Advanced exposition (research surveys, etc.)
15-03 Historical

15-04 Explicit machine computation and programs (not the theory of computation or programming)

15A03 Vector spaces, linear dependence, rank

Hoffman, Alan J.
Weil, Roman L., Jr.
Thompson, Gerald L.

1S

$1 \mathrm{P}$

15A06 Linear equations

Swan, Richard G.

$1 \mathbf{P}$

15A09 Matrix inversion, generalized inverses

15A12 Conditioning of matrices [See also 65F35.]

15A15 Determinants, permanents, other special matrix functions

Sinkhorn, Richard
Minc, Henryk
O'Neil, Patrick Eugene
Brown, William G.
Brenner, Joel Lee
Hartfiel, D. J.
Gibson, Peter M.
Salehi, Habib
Fiedler, Miroslav
Brenner, Joel Lee

$1 \mathrm{P}$

$1 \mathrm{P}$

O'Neil, Patrick Eugene 2P

Brown, William G. IP

Brenner, Joel Lee IP

$2 S$

$2 \mathrm{P}$

Brenner, Joel Lee $2 S$

15A18 Eigenvalues and eigenvectors

Brand, Louis

Ballantine, Charles S.

Weil, Roman L., Jr.

Thompson, Gerald L.

Schaefer, Helmut $\mathrm{H}$.

Fiedler, Miroslav

$1 \mathrm{P}$

1P

1S

1S

1P

15A21 Canonical forms, reductions, classification

$\begin{array}{ll}\text { Hartfiel, D. J. } & 1 \mathrm{P} \\ \text { Sinkhorn, Richard } & 2 \mathrm{~S} \\ \text { Hartfiel, D. J. } & 2 \mathrm{P} \\ \text { Hartfiel, D. J. } & 3 \mathrm{P}\end{array}$

15A24 Matrix equations and identities

Marden, Morris

Crabtree, Douglas E.

Salehi, Habib

$1 \mathbf{P}$
$1 \mathbf{P}$

15A27 Commutativity

15A30 Algebraic systems of matrices [See also 16A42, 20Gxx, 20Hxx.]

Weil, Roman L., Jr.

Thompson, Gerald L.

Au-yeung, Yik-hoi

Hartfiel, D. J.

15A33 Matrices over special rings (quaternions, finite fields, etc.)

Gupta, Ram Niwas

Au-yeung, Yik-hoi

$1 \mathrm{P}$ 
15A36 Matrices of integers [See also 10M20.]

Minc, Henryk

Hartfiel, D. J.

15A39 Linear inequalities

Flatto, Leopold

Levin, Simon A.

Block, Henry David

$1 \mathrm{P}$
$1 \mathrm{P}$
$1 \mathrm{P}$

15A42 Inequalities involving eigenvalues and eigenvectors

Salehi, Habib

Gellar, Ralph

15A45 Miscellaneous inequalities involving matrices

Đokovic̀, Dragomir Ż.

$2 \mathrm{P}$

15A48 Positive matrices and their generalizations

Đokovic̀, Dragomir Ż.

Taylor, Gerald D.

Salehi, Habib

Hartfiel, D. J.

Schaefer, Helmut $\mathbf{H}$.

Salehi, Habib

Gellar, Ralph

15A51 Stochastic matrices

Sinkhorn, Richard

DeMarr, Ralph E.

Đokovič, Dragomir Ž.

Yeh, R. Z.

Sinkhorn, Richard

15A54 Matrices over function rings in one or more variables

Salehi, Habib

15A57 Other types of matrices

(hermitian, skew-hermitian, etc.)

Radjavi, Heydar

Brand, Louis

Au-yeung, Yik-hoi

Au-yeung, Yik-hoi

15 A60 Norms of matrices, applications of functional analysis to matrix theory [See also 65F35, 65J05.]

McCoy, R. A.
Salehi, Habib

Salehi, Habib

$1 \mathrm{P}$

2S

15A63 Quadratic and bilinear forms, inner products

James, D. G.

Au-yeung, Yik-hoi

Đokovic̀, Dragomir Ž

$1 P$
$1 P$
$2 P$

15A66 Clifford algebras

15A69 Multilinear algebra, tensor products

Huq, Syed A
15A72 Vector and tensor algebra, theory of invariants

$\begin{array}{ll}\text { Huq, Syed A. } & 1 S \\ \text { Sinkhorn, Richard } & 2 \mathrm{P}\end{array}$

15A75 Exterior algebra, Grassmann algebras

15A78 Other algebras built from modules

Barshay, Jacob

$1 \mathbf{P}$

16-XX ASSOCIATIVE RINGS

AND ALGEBRAS \{For the commutative case, see $13-\mathrm{XX}$.

16 - 00 Difficult to classify at the second level (must also be assigned at least one other classification number in this section)

16-01 Elementary exposition

(collegiate level)

16-02 Advanced exposition (research surveys, etc.)

16-03 Historical

16-04 Explicit machine computation and programs (not the theory of computation or programming)

16A02 Integral domains, unique factorization domains (noncommutative)

$\begin{array}{ll}\text { Van de Water, Arthur } & 1 \mathrm{P} \\ \text { Johnson, R. E. } & 2 \mathrm{P} \\ \text { Beauregard, Raymond A. } & \text { 1P } \\ \text { Beauregard, Raymond A. } & \text { 2P }\end{array}$

16A04 Noncommutative principal ideal rings, rings with a division algorithm

Brungs, Hans-Heinrich

$1 \mathbf{P}$

16A06 Free algebras, free ideal rings

(firs) and their generalizations [See also tensor algebra in 15A72.]

Williams, R. E.

Klein, Abraham

Fisher, J. L.

16A08 Rings of quotients, noncommutative localization

Neggers, Joseph

Chwe, Byoung-song

Teply, Mark L.

Brungs, Hans-Heinrich

Hutchinson, John J.

Fisher, J. L.

P

10 Noncommutative local rings

Lenzing, Helmut

16A12 Prime and semiprime rings

Martindale, Wallace S., III

1S 
Armendariz, Efraim P.

Griffith, Phillip A

Robson, J. C.

Koh, Kwangi

Bhattacharya, P. B.

Jain, S. K.

Koh, Kwangi

16A14 Noncommutative analogues of Dedekind and Prüfer domains

16A16 Separable algebras, Azumaya algebras and their generalizations [See also $13 \mathrm{~A} 20$.]

Brown, William C

Ingraham, Edward C.

16A18 Orders, arithmetic in / lgebras

Jacobinski, $\mathbf{H}$

Jacobinski, $\mathbf{H}$.

Riley, John A

1P

16A20 Primitive and semiprimitive rings

Martindale, Wallace S., III 2P

16A21 Radical theory

Leavitt, William G.

Haimo, Franklin

Leavitt, William $\mathbf{G}$.

Heinicke, A. G.

Zelmanowitz, Julius Martin

Ware, Roger

Jenkins, Terry L.

Kreiling, Daryl

LaTorre, D. R.

Rich, Michael

$1 \mathrm{~S}$
$1 \mathrm{P}$
$2 \mathrm{P}$
$1 \mathrm{P}$
$1 \mathrm{P}$
$1 \mathrm{P}$
$1 \mathrm{P}$
$1 \mathrm{P}$
$2 \mathrm{~S}$
$2 \mathrm{~S}$

16A22 Nil, nilpotent and radical rings

$\begin{array}{ll}\text { Chao, Chong-yun } & \text { IP } \\ \text { Klein, Abraham } & \text { 1P } \\ \text { Bhattacharya, P. B. } & \text { 1P } \\ \text { Jain, S. K. } & \text { IP }\end{array}$

16A24 Hopf algebras, algebraic theory [See also 57F05.]

Larson, Richard Gustavus

May, J. Peter

Hochschild, G

Radford, David E.

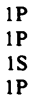

16A26 Group rings [See also 20C05.]

Gentile, Enzo R.

Passman, D. S.

Hill, E. T.

Woods, S. M.

Benard, Mark

$1 \mathrm{P}$
$2 \mathrm{P}$
$1 \mathrm{~S}$
$1 \mathrm{P}$
$1 \mathrm{P}$

16A28 Rings with involution [See also 46Kxx.]

Martindale, Wallace S., III

Montgomery, Susan

Montgomery, Susan

16A30 von Neumann regular rings and their generalizations [See also 06A30.]

Faudree, R. J.

Wiegand, Roger 

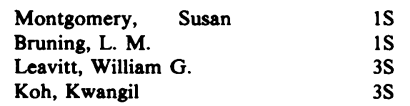

16A49 Duality theory

16A50 Projective and flat modules and generalizations

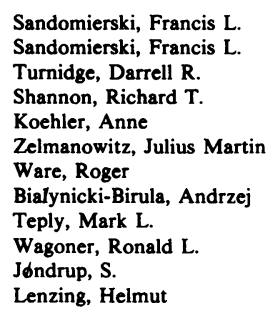

16A52 Injective modules, self-injective rings and generalizations

$$
\begin{aligned}
& \text { Gentile, Enzo R. } \\
& \text { Armendariz, Efraim P. } \\
& \text { Koehler, Anne } \\
& \text { Van de Water, Arthur } \\
& \text { Sandomierski, Francis L. } \\
& \text { Megibben, Charles K. } \\
& \text { Wagoner, Ronald L. }
\end{aligned}
$$

16A54 Grothendieck groups of rings, $\mathrm{K}$-theory of noncommutative rings [See also 18F25.]

$$
\text { Gersten, Stephen M. }
$$

16A56 Extension theory

\section{Nobusawa, Nobuo}

Kohls, Carl W.

16A58 Deformation theory of rings and algebras [See also 13D10, 32Gxx.]

16A60 Homological dimension

$\begin{array}{ll}\text { Sandomierski, Francis L. } & 2 \mathrm{P} \\ \text { Megibben, Charles K. } & 1 \mathrm{P} \\ \text { Jacobinski, H. } & 2 \mathrm{~S}\end{array}$

16A62 Homological methods [For cohomology of algebras and rings, see 18H15.]

$$
\begin{aligned}
& \text { Teply, Mark L. } \\
& \text { Alin, J. S. }
\end{aligned}
$$

16A64 Modules and representations

$$
\begin{aligned}
& \text { Quillen, Daniel G. } \\
& \text { Chwe, Byoung-song } \\
& \text { Neggers, Joseph } \\
& \text { Koh, Kwangil } \\
& \text { Sandomierski, Francis L. } \\
& \text { Bialynicki-Birula, Andrzej } \\
& \text { Alin, J. S. } \\
& \text { Leavitt, William G. } \\
& \text { Bruning, L. M. } \\
& \text { Wagoner, Ronald L. } \\
& \text { Bang, Chang Mo } \\
& \text { Warner, Seth }
\end{aligned}
$$

sec


Witthoft, William G.

2P

17 A10 Commutative power-associative

Kass, Seymour

Witthoft, William G.

$1 \mathrm{~S}$

$2 \mathrm{~S}$

17A15 Noncommutative Jordan algebras

Ravisankar, T. S.

Goldman, Jerry I.

17A20 Flexible algebras

17A25 Nodal algebras

Ravisankar, T. S.

Goldman, Jerry I.

Rich, Michael

17A30 Algebras satisfying other identities

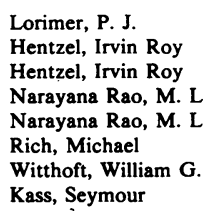

17A99 None of the above, but in this section

Leavitt, William G.

Ravisankar, T. S.

Leavitt, William G.

Rich, Michael
17B35 Universal enveloping algebras

Quillen, Daniel G.

1S

17 B40 Automorphisms, derivations, other operators

$\begin{array}{ll}\text { Schenkman, Eugene } & \text { 2P } \\ \text { Ravisankar, T. S. } & \text { 2P } \\ \text { Putcha, Mohan S. } & \text { 1P } \\ \text { Hochschild, G. } & \text { IS }\end{array}$

17B45 Lie algebras of linear algebraic groups [See also 14Lxx and 20Gxx.]

Hochschild, G.

17 B50 Lie p-algebras and bialgebras (Hopf algebras) of formal groups [See also 14L05, 16A24.]

17B55 Homological methods in Lie algebra [For homology of Lie algebras, see $18 \mathrm{H} 25$.

17 B60 Lie rings associated with other structures (associative, Jordan, etc.) [See also $15 \mathrm{~A} 30,16 \mathrm{~A} 68,17 \mathrm{C} 40,17 \mathrm{C} 50$.

17 B65 Infinite dimensional Lie algebras [See also 22E65.]

17B99 None of the above, but in this section

17Cxx Jordan algebras (commutative)

17C05 Identities

McCrimmon, Kevin

2P

$17 \mathrm{C} 10$ Structure theory

Tsai, Chester E.

$1 \mathbf{P}$

17C15 Representations

17C20 Simple, semisimple algebras

$17 \mathrm{C} 25$ Universal enveloping algebras

$17 \mathrm{C} 30$ Automorphisms, derivations, other operators

17B10 Representations, algebraic theory (weights)
Wallach, Nolan $\mathbf{R}$.

Lemire, F. W.
17B15 Representations, analytic theory

17B20 Simple, semisimple, reductive algebras (roots)

Wallach, Nolan $\mathbf{R}$.

Ravisankar, T. S.

Lemire, F. W.

17B25 Exceptional algebras

17B30 Solvable, nilpotent algebras

Quillen, Daniel G.

Chao, Chong-yun

Stitzinger, Ernest L.

Putcha, Mohan S.
17C35 Formally real domains of positivity [See also $32 \mathrm{Nxx}$.]

Resnikoff, H. L.

$1 \mathrm{P}$

17C40 Exceptional Jordan algebras and associated Lie groups

17C45 Homological methods in Jordan algebras [For homology of Jordan algebras, see 18H30.]

$17 \mathrm{C50}$ Jordan rings associated with other structures [See also 16A68, 17B60.]

$17 C 99$ None of the above, but in this section

17D05 Alternative rings 


$\begin{array}{ll}\text { Kleinfeld, Erwin } & \text { 1P } \\ \text { Kleinfeld, Margaret Humm } & \text { 1P } \\ \text { Hentzel, Irvin Roy } & \text { 1P } \\ \text { Hentzel, Irvin Roy } & \text { 2P } \\ \text { Jenkins, Terry L. } & 1 P \\ \text { Kreiling, Daryl } & \text { 1P }\end{array}$

17E05 Other nonassociative rings and algebras

$\begin{array}{lr}\text { Leavitt, William G. } & 1 \mathrm{P} \\ \text { Ravisankar, T. S. } & 2 \mathrm{P} \\ \text { Hentzel, Irvin Roy } & 1 \mathrm{P} \\ \text { Hentzel, Irvin Roy } & 2 \mathrm{P} \\ \text { Rich, Michael } & 1 \mathrm{P} \\ \text { Jenkins, Terry L. } & 1 \mathrm{~S} \\ \text { Kreiling, Daryl } & 1 \mathrm{~S} \\ \text { Thedy, Armin } & 1 \mathrm{P} \\ \text { Rich, Michael } & 2 \mathrm{P} \\ \text { XX CATEGORY } & \\ \text { XXRY, HOMOLOGICAL } \\ \text { TORYRA }\end{array}$

\section{ALGEBRA}

$18-00$ Difficult to classify at the second level (must also be assigned at least one other classification number in this section)

$18-01$ Elementary exposition (collegiate level)

18-02 Advanced exposition (research surveys, etc.)

18-04 Explicit machine computation and programs (not the theory of computation or programming)

18Axx General theory of categories and functors

18A05 Definitions, generalizations

18A10 Graphs, groupoids, neofunctors [See also 20Lxx.]

18A 15 Foundations, relations to logic and deductive systems [See also 02-XX.]

18A20 Epimorphisms, monomorphisms, special classes of morphisms, null morphisms, factorization (bicategories)

$\begin{array}{ll}\text { Noll, Walter } & \text { IP } \\ \text { Klein, Aaron } & \text { is }\end{array}$

18A25 Functor categories, comma categories

18A30 Limits and colimits (products, sums, pushouts, fiber products, equalizers, kernels, ends and coends, etc.)

18A35 Complete categories, completions 18A40 Adjoint functors (representable functors, universal constructions, reflexive subcategories, etc.), constructions of adjoints (Kan extensions, etc.)

Davis, Robert

18A99 None of the above, but in this section

Klein, Aaron

$1 \mathrm{P}$

$18 \mathrm{Bxx}$ Special categories

18B05 Category of sets, characterizations

Davis, Robert

$18 \mathrm{~B} 10$ Category of relations, additive relations, semigroups [See also 20Mxx.]

Huq, Syed A. Wyler, Oswald

$1 \mathrm{P}$

18B15 Embedding theorems, universal categories [See also 18E20.]

18B20 Categories of machines, automata, operative categories [See also $02 \mathrm{~F} 10$, 68A25, 94A30, 94A35.]

18B99 None of the above, but in this section

Hager, Anthony w.

$2 S$

$18 \mathrm{Cxx}$ Categories and algebraic theories

$18 \mathrm{C} 05$ Equational categories [See also 02-XX.]

Huq, Syed A.

$18 \mathrm{C} 10$ Theories (e.g. algebraic theories), structure, and semantics

Davis, Robert

Huq, Syed A

2P

iS

$18 \mathrm{C} 15$ Triples (= standard construction, monad or triad), algebras for a triple, homology and derived functors for triples [See.also 18Gxx.]

Davis, Robert

IP

$18 \mathrm{C} 99$ None of the above, but in this section

18Dxx Categories with structure

18D05 Double categories, 2-categories, bicategories, hypercategories

$18 \mathrm{D} 10$ Monoidal categories (= multiplicative categories)

18D15 Closed categories (closed monoidal and cartesian closed categories, etc.)

18D20 Enriched categories (over closed or monoidal categories) 
18D25 Strong functors, strong adjunctions

18D30 Fibered categories

18D35 Structured objects in a category (group objects, etc.)

$$
\text { Hall, C. E. }
$$

18D99 None of the above, but in this section

\section{Exx Abelian categories}

18E05 Preadditive, additive categories

$18 \mathrm{E} 10$ Exact categories, abelian categories

$18 \mathrm{E} 15$ Grothendieck categories

$18 \mathrm{E} 20$ Embedding theorems [See also 18B10.]

18 E25 Derived functors and satellites

$18 \mathrm{E} 30$ Derived categories, triangulated categories

18 E35 Localization of categories

$18 \mathrm{E} 40$ Torsion theories, radicals

$$
\begin{array}{ll}
\text { Bernhardt, Robert L. } & 1 \mathrm{P} \\
\text { Teply, Mark L. } & 2 \mathrm{~S}
\end{array}
$$

18 E99 None of the above, but in this section

Noll, Walter

18Fxx Categories and geometry

$18 \mathrm{~F} 05$ Local categories and functors

$18 \mathrm{~F} 10$ Grothendieck topologies [See also 14F20.]

18 F15 Abstract manifolds and fiber bundles [See also 55Fxx, 57Bxx.]

18 F20 Presheaves and sheaves [See also 14F05, 32C35, 32L10, 55B30.]

18F25 Algebraic K-theory [See also 13D15, 14F15, 16A54.]

Evans, E. Graham, Jr Gersten, Stephen M.

18F30 Grothendieck groups [See also 13D15, 16A54.]

18 F99 None of the above, but in this section

\section{Gxx Homological algebra}

18 G05 Projectives and injectives [See also $13 \mathrm{C} 10,16 \mathrm{~A} 50,16 \mathrm{~A} 52$.] $\begin{array}{ll}\text { Megibben, Charles K. } & \text { is } \\ \text { Teply, Mark L. } & \text { is } \\ \text { Wagoner, Ronald L. } & \text { is }\end{array}$

18G10 Resolutions; derived functors [See also 18E25.]

18 G15 Ext and Tor, generalizations, Künneth formula [See also 55J25.]

18G20 Homological dimension [See also 13Dxx, 16A62.]

Landweber, Peter $\mathrm{S}$.

$1 \mathrm{P}$

18G25 Relative homological algebra, projective classes

18 G30 Simplicial sets, simplicial objects (in a category) [See also 55J10.]

18 G35 Chain complexes [See also 18E30, 55J15.]

Fleury, Patrick J.

$1 \mathbf{P}$

18G40 Spectral sequences, hypercohomology [See also 55Hxx.]

18G99 None of the above, but in this section

$18 \mathrm{Hxx}$ Cohomology of specified algebraic systems

18H05 General methods; models [See also 18E25, 18G25.]

$18 \mathrm{H} 10$ Cohomology and homology of groups [See also 20Jxx.]

$\begin{array}{ll}\text { Ribes, Luis } & \text { 1P } \\ \text { Rinehart, George S. } & \text { IP } \\ \text { Formanek, Edward } & \text { IP } \\ \text { Landweber, Peter S. } & \text { IP } \\ \text { Sumners, D. W. } & \text { IP }\end{array}$

$18 \mathrm{H} 15$ Cohomology and homology of algebras and rings [See also 16A62.]

Bialynicki-Birula, Andrzej $\quad 1 \mathrm{P}$

$18 \mathrm{H} 20$ Cohomology and homology of commutative rings [See also 13Dxx.]

$\begin{array}{ll}\text { Greenleaf, Newcomb } & \text { IP } \\ \text { Fleury, Patrick J. } & \text { IP }\end{array}$

$18 \mathrm{H} 25$ Cohomology of Lie algebras [See also 17B55.]

$18 \mathrm{H} 30$ Cohomology of Jordan algebras [See also 17C45.]

$18 \mathrm{H} 35$ Cohomology of other nonassociative algebras [See also 17-XX.]

$18 \mathrm{H} 40$ Cohomology of other algebraic systems

Westman, Joel J.

$1 \mathrm{P}$

18 H99 None of the above, but in this 
section

\section{0-XX GROUP THEORY}

\section{AND GENERALIZATIONS}

\{For ordered groups, see 06A55. \}

20-00 Difficult to classify at the second level (must also be assigned at least one other classification number in this section)

20-01 Elementary exposition (collegiate level)

20-02 Advanced exposition (research surveys, etc.)

20-03 Historical

20-04 Explicit machine computation and programs (not the theory of computation or programming)

\section{Axx Foundations}

20A05 Axiomatics and elementary properties

20A10 Metamathematical considerations [For word problems, see 20F10.]

20A99 None of the above, but in this section

20Bxx Finite permutation groups

20B05 General theory

20B10 Characterization theorems

20B15 Uniprimitive groups

20B20 Multiply transitive groups

20B25 Automorphism groups of algebraic, geometric, or combinatorial structures [See also 05Bxx, 20G40, $20 \mathrm{H} 30,50-\mathrm{XX}$.]

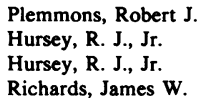

20B99 None of the above, but in this section

20Cxx Representation theory of finite groups

$20 \mathrm{C05}$ Group rings and their modules [See also 16A26.]

Faudree, R. J.

Passman, D. S.

Gentile, Enzo R.

Reiner, Irving

Lam, Tsit-yuen

Passman, D. S.

Coleman, Donald B.
Passman, D. S.

Hill, E. T.

Woods, S. M.

Hill, E. T.

Benard, Mark

Fields, K. L.

Fleury, Patrick J.

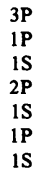

20C10 Integral representations

$\begin{array}{ll}\text { Grove, Larry C. } & \text { IP } \\ \text { Bialynicki-Birula, Andrzej } & \text { is }\end{array}$

20C15 Ordinary representations and characters

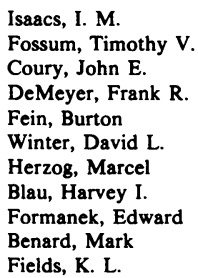

20C20 Modular representations and characters

Fein, Burton

Hill, E. T.

$20 C 25$ Projective representations and multipliers

20C30 Representations of symmetric groups and other special groups

20C35 Applications to physics

$20 \mathrm{C} 99$ None of the above, but in this section

\section{Dxx Abstract finite groups}

20D05 Simple groups [See also 20G40.]

Herzog, Marcel Herzog, Marcel

20D10 Solvable groups

Bauman, Steven F.

Isaacs, I. $\mathbf{M}$.

Basmaji, B. G.

Faudree, R. J.

Randolph, J. W.

Parker, Donald B.

Dornhoff, Larry

Schmidt, H. J., Jr.

DeMeyer, Frank R.

Inagaki, Nobuo

Yen, Ti

Kotzen, Marshall

Faudree, R. J.

Gilman, Robert

Chambers, Graham A

Richards, James W.

20D15 Nilpotent groups, p-groups

Davitt, Richard M.

Jain, N. C. 
Macdonald, Ian D. Landweber, Peter S.

Faudree, R. J.

20D20 Sylow subgroups, $\pi$-groups

Richen, Forrest

Inagaki, Nobuo

Landweber, Peter S.

$20 D 25$ Special subgroups (Frattini, Fitting, etc.)

Randolph, J. W.

Stitzinger, Ernest L.

Mukherjee, N. P.

Macdonald, Ian D.

20D30 Series and lattices of subgroups

Richen, Forrest

Kotzen, Marshall

20D35 Subnormal subgroups

Schenkman, Eugene

20 D40 Products of subgroups

Parker, Donald B.

Reilly, Norman R.

Gilman, Robert

Friesen, Donald K.

20D45 Automorphisms

Schenkman, Eugene

Davitt, Richard M.

Jain, N. C.

Richards, James W.

Formanek, Edward

Otto, Albert D.

Davitt, Richard M

20D99 None of the above, but in this section

Bernstein, Herbert J.

\section{Exx Infinite groups}

20E05 Free nonabelian groups

Karrass, Abraham

Burns, R. G.

$20 \mathrm{E} 10$ Varieties of groups

Hickin, Kenneth $\mathrm{K}$.

Schwabauer, Robert

20E15 Chains of subgroups (solvable groups, nilpotent groups, etc.)

$\begin{array}{lr}\text { Hickin, Kenneth K. } & \text { 1P } \\ \text { Faudree, R. J. } & \text { IS } \\ \text { Hill, Paul D. } & \text { 2P } \\ \text { Hursey, R. J., Jr. } & \text { IS } \\ \text { Chandy, A. John } & \text { IP }\end{array}$

20 20 Special subgroups other than commutator types

20E25 Local properties, residual properties

Faudree, R. J.

$\begin{array}{ll}\text { Stebe, Peter } & 1 P \\ \text { Hursey, R. J., Jr. } & \text { iS }\end{array}$

20E30 Free products, generalized properties

$\begin{array}{ll}\text { Billis, M. J. } & \text { IP } \\ \text { Hirshon, R. } & \text { 1P } \\ \text { Gregorac, Robert J. } & \text { 1P } \\ \text { Baumslag, Gilbert } & \text { 1P } \\ \text { Karrass, Abraham } & 3 P\end{array}$

20E35 Representation in associative rings, Lie rings, combinatorial structures, etc.

$\begin{array}{ll}\text { Faudree, R. J. } & \text { 1P } \\ \text { Bialynicki-Birula, Andrzej } & \text { 1P }\end{array}$

20E40 Fundamental groups, etc. [See also 55A05, 57Exx.]

Tollefson, Jefferey $L$.

Birman, Joan S.

20E99 None of the above, but in this section

20Fxx General groups, finite or infinite

20 F05 Generators and relations

$\begin{array}{ll}\text { Hochster, M. } & \text { 2P } \\ \text { Emerson, William R. } & \text { 1P } \\ \text { Grove, Larry C. } & \text { 2P } \\ \text { Benson, Clark T. } & \text { 1P } \\ \text { Formanek, Edward } & \text { 1S }\end{array}$

20F10 Word problems

$\begin{array}{ll}\text { Appel, K. I. } & \text { 1P } \\ \text { Lipschutz, Seymour } & \text { 1P } \\ \text { Stebe, Peter } & \text { 1P } \\ \text { McCool, James } & \text { IP }\end{array}$

20F15 Structure theorems

$\begin{array}{ll}\text { Whittemore, Alice } & \text { 1P } \\ \text { Cossey, John } & \text { 1P } \\ \text { Gregorac, Robert J. } & \text { 1P }\end{array}$

20F20 Limits, profinite groups

20F25 Extensions, wreath products, other compositions

$\begin{array}{ll}\text { Simon, Hermann } & \text { 1P } \\ \text { Amberg, Bernhard } & \text { 1P } \\ \text { Scott, W. R. } & \text { 2P }\end{array}$

20F30 Subgroup lattices, maximal subgroups, subnormal subgroups, etc.

Whittemore, Alice Cossey, John

$1 \mathrm{P}$

20F35 Commutator subgroups and their generalizations

20F40 Associated Lie structures

Schenkman, Eugene

Stitzinger, Ernest L. 
Putcha, Mohan S.

20F45 Engel conditions

Faudree, R. J.

Putcha, Mohan S.

is

20F50 Periodic groups

Gupta, Narain Datt

20F55 Automorphisms

Schenkman, Eugene

Conlon, Lawrence

Chandy, A. John

$1 \mathrm{~S}$

2P

$1 \mathrm{P}$
$1 \mathrm{P}$

20F99 None of the above, but in this section

20Gxx Linear algebraic groups (classical groups) See also 10C30, 14-XX.

20G05 Representation theory [For infinite dimensional representations, see 22E50.]

20G10 Cohomology theory

20G15 Linear algebraic groups over arbitrary fields

$$
\text { Hochschild, } \mathbf{G} \text {. }
$$

$1 \mathrm{P}$

20G20 Linear algebraic groups over the reals, the complexes, the quaternions

Blau, Harvey I.

Hochschild, $\mathbf{G}$

$1 \mathrm{P}$

20G25 Linear algebraic groups over local fields and their integers

20G30 Linear algebraic groups over global fields and their integers

$$
\text { Weisman, Carl S. }
$$

20G35 Linear algebraic groups over adèles and other rings and schemes

$$
\text { Weisman, Carl S. }
$$

$1 \mathbf{P}$

20 G40 Linear algebraic groups over finite fields

20G45 Applications to physics; explicit representations

20G99 None of the above, but in this section

\section{Hxx Other groups of matrices}

20H05 Unimodular groups, congruence subgroups

Mason, A. W.

$1 \mathrm{P}$

20H10 Fuchsian groups and their generalizations [See also 30A58, 32Nxx.]

$\begin{array}{ll}\text { Mason, A. W. } & \text { IP } \\ \text { MacLachlan, C. } & \text { IS } \\ \text { Feuer, R. D. } & \text { IP }\end{array}$

$20 \mathrm{H} 15$ Other geometric groups, including crystallographic groups [See also 50-XX.]

$\begin{array}{ll}\text { Grove, Larry C. } & 2 \mathrm{P} \\ \text { Benson, Clark T. } & 1 \mathrm{P}\end{array}$

$20 \mathrm{H} 20$ Other matrix groups over fields

Dornhoff, Larry

$1 \mathrm{P}$

$20 \mathrm{H} 25$ Other matrix groups over rings

$20 \mathrm{H} 30$ Other matrix groups over finite fields

20 H99 None of the above, but in this section

20Jxx Connections with homological algebra and category theory

20J05 Homological methods in group theory

Rinehart, George S.

Formanek, Edward

Landweber, Peter $\mathrm{S}$

1P

1P

$20 \mathrm{~J} 10$ Groups arising as cohomology groups

20J15 Category of groups

$20 J 99$ None of the above, but in this section

Ribes, Luis

$1 \mathrm{~S}$

20Kxx Abelian groups

20K05 Finitely generated groups

Karrass, Abraham

Richards, James W.

20K10 Torsion groups

Winthrop, Joel

Cutler, Doyle O.

Griffith, Phillip A

Cutler, Doyle $O$.

Hill, Paul D.

Stringall, Robert W.

20K15 Torsion free groups, finite rank

20K20 Torsion free groups, infinite rank

Beaumont, Ross A.

Kohls, Carl W.

Cornelius, E. F., Jr.

$1 \mathrm{P}$

$1 P$

20K25 Direct sums, direct products, etc.

Walker, Elbert A.
Richman, Fred

Bang, Chang Mo

$1 \mathrm{P}$

20K30 Automorphisms, 
homomorphisms, endomorphisms

Hill, Paul D.

Tarwater, J. Dalton

Hill, Paul D.

$$
\begin{aligned}
& 1 P \\
& 1 P \\
& 3 P
\end{aligned}
$$

20K35 Extensions

Griffith, Phillip A.

Hill, Paul D.

20K40 Homological and categorical methods [See also 18H10.]

20K45 Topological methods

Armacost, David Lee

1P

20K99 None of the above, but in this section

Sperry, P. L.

$1 \mathrm{P}$

20Lxx Groupoids (small categories in which all morphisms are isomorphisms)

20L05 General theory

Grätzer, György

Padmanabhan, $\mathbf{R}$.

20L10 Connections with group theory

20L15 Connections with topology

20L99 None of the above, but in this section

\section{Mxx Semigroups}

20M05 Free semigroups, generators and relations, word problem

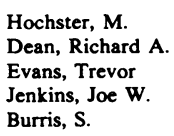

$2 \mathrm{P}$
$1 \mathrm{P}$
$1 \mathrm{P}$
$3 \mathrm{~S}$
$1 \mathrm{P}$

20M10 Structure theory, ideals, idempotents, etc.

$\begin{array}{ll}\text { Ferguson, Edward N. } & 1 \mathrm{~S} \\ \text { Petrich, Mario } & 1 \mathrm{P} \\ \text { Clark, C. E. } & 1 \mathrm{P} \\ \text { Carruth, J. H. } & 1 \mathrm{P} \\ \text { Stepp, James W. } & 2 \mathrm{~S} \\ \text { Rigelhof, Roger } & 2 \mathrm{P} \\ \text { Iyengar, H. R. Krishna } & 1 \mathrm{P} \\ \text { LaTorre, D. R. } & 2 \mathrm{P}\end{array}$

20M15 Mappings of semigroups

$\begin{array}{ll}\text { Johnson, R. E. } & 1 \mathrm{P} \\ \text { Schelp, R. H. } & 1 \mathrm{P} \\ \text { Gudder, Stanley P. } & 2 \mathrm{P}\end{array}$

20M20 Semigroups of transformations, etc. [See also 47D05.]

20M25 Semigroup rings, multiplicative semigroups of rings

20M30 Representation of semigroups
20M35 Semigroups in automata theory, linguistics, etc. [See also 02F10.]

Bednarek, Alexander R.

20M99 None of the above, but in this section

$\begin{array}{ll}\text { Chrislock, J. L. } & \text { 1P } \\ \text { Lin, You-feng } & 1 \mathrm{P} \\ \text { Schwabauer, Robert } & 1 \mathrm{P} \\ \text { Jenkins, Joe W. } & 3 \mathrm{~S}\end{array}$

20Nxx Other generalizations of groups

20N05 Loops, quasigroups [See also 05Bxx.]

Lindner, Charles $\mathrm{C}$. $2 \mathrm{P}$

20N10 Ternary systems (heaps, semiheaps, heapoids, etc.)

$20 \mathrm{~N} 15$ n-ary systems

20N99 None of the above, but in this section

\section{2-XX TOPOLOGICAL} GROUPS, LIE GROUPS (For transformation groups, see $54 \mathrm{H} 15$, 57Exx, $58-\mathrm{XX}$. For abstract harmonic analysis, see $43-\mathrm{XX}$. 22-00 Difficult to classify at the second level (must also be assigned at least one other classification number in this section)

22-01 Elementary exposition (collegiate level)

22-02 Advanced exposition (research surveys, etc.)

22-03 Historical

22--04 Explicit machine computation and programs (not the theory of computation or programming)

22Axx Topological algebraic systems (For topological rings and fields, see $12 \mathrm{Jxx}, 13 \mathrm{Jxx}, 16 \mathrm{~A} 80$; for dual spaces of operator algebras and topological groups, see 46L05.\} 22A05 Structure of general topological groups

$\begin{array}{ll}\text { Hudson, Sigmund N. } & \text { 1P } \\ \text { Atalla, Robert E. } & 2 \mathrm{P} \\ \text { Hall, C. E. } & \text { 1P } \\ \text { Bagley, Robert W. } & 2 \mathrm{P} \\ \text { Lau, K. K. } & \text { 1P }\end{array}$


22A10 Analysis on general topological groups

$$
\begin{aligned}
& \text { Pym, John S. } \\
& \text { Peterson, H. LeRoy } \\
& \text { Atalla, Robert E. }
\end{aligned}
$$

22 A 15 Structure of topological semigroups

Edmondson, Don E.
Rothman, Neal J.
Hildebrant, John A.
Borrego, Joseph T.
Ferguson, Edward N.
Jenkins, Joe W.
Hanson, T. H. McH.
Brown, Robert F.
Janoš, Ludvík
Jenkins, Joe W.
Clark, C. E.
Carruth, J. H.
Fulp, Ronald O.
Stepp, James W.
Wilder, B .E.

22A20 Analysis on topological semigroups

$$
\begin{aligned}
& \text { Rothman, Neal J. } \\
& \text { Jenkins, Joe W. } \\
& \text { Jenkins, Joe W. }
\end{aligned}
$$

22A25 Representations of general topological groups and semigroups

Madell, Robert L.

22A30 Representations of other topological algebraic systems

Warner, Seth

22A99 None of the above, but in this section

Jamison, Benton
Orey, Steven

Madell, Robert L.

22Bxx Locally compact abelian groups (LCA groups)

22B05 General properties and structure of LCA groups

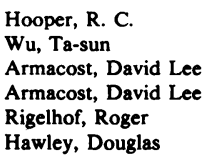

Hooper, R. C.

Wu, Ta-sun

Armacost, David Lee

Armacost, David Lee

Rigelhof, Roger

Hawley, Douglas

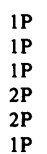

22B10 Structure of group algebras of

LCA groups

Graham, Colin C.

22B99 None of the above, but in this section

Coven, Ethan $\mathbf{M}$.

Reddy, William L.

Wang, Ju-kwei

\section{C05 Compact groups}

\author{
Lazarov, Connor \\ Vasquez, Alphonse T \\ Dugundji, John \\ Curtis, Morton L. \\ Coury, John E. \\ Wood, Geoffrey V. \\ Poguntke, Detlev \\ Wolf, Joseph A. \\ Wilcox, Howard J. \\ Joffe, A. D. \\ Elliott, George A.
}

22Dxx Locally compact groups and their algebras

22D05 General properties and structure of locally compact groups

$\begin{array}{ll}\text { Hudson, Sigmund N. } & \text { IP } \\ \text { Wu, Ta-sun } & 2 \mathrm{P} \\ \text { Jenkins, Joe W. } & 2 \mathrm{P} \\ \text { Jenkins, Joe W. } & 3 \mathrm{P} \\ \text { Bagley, Robert W. } & 2 \mathrm{P} \\ \text { Lau, K. K. } & 1 \mathrm{P} \\ \text { Schochetman, Irwin } & 2 \mathrm{P} \\ \text { Milnes, Paul } & 1 \mathrm{~S} \\ \text { Mosak, Richard D. } & 1 \mathrm{P}\end{array}$

22D10 Unitary representations of locally compact groups

$\begin{array}{ll}\text { Baggett, Larry } & \text { IP } \\ \text { Coury, John E. } & \text { IP } \\ \text { Schochetman, Irwin } & \text { 2S }\end{array}$

22D12 Other representations of locally compact groups

$\begin{array}{ll}\text { Vasquez, Alphonse T. } & \text { is } \\ \text { Lazarov, Connor } & \text { is }\end{array}$

22D15 Group algebras of locally compact groups

$\begin{array}{ll}\text { Rigelhof, Roger } & \text { IP } \\ \text { Mosak, Richard D. } & \text { IP } \\ \text { Flory, Volker } & \text { IP }\end{array}$

22D20 Representations of group algebras

$\begin{array}{ll}\text { Rigelhof, Roger } & \text { IP } \\ \text { Flory, Volker } & \text { IP }\end{array}$

22D25 $\mathrm{C}^{*}$-algebras and $\mathrm{W}^{*}$-algebras arising from group representations

Baggett, Larry

Milnes, Paul

Flory, Volker

S

22D30 Induced representations

Schochetman, Irwin 2S

Busby, Robert C. 1 .

22D35 Duality theorems

Milnes, Paul

22D40 Ergodic theory on groups [See also 28A65.]

Schochetman, Irwin

2P 
Westman, Joel J.

\author{
$1 \mathrm{P}$
}

22D45 Automorphism groups of locally compact groups

Joffe, A. D.

$22 D 99$ None of the above, but in this section

Wong, James C. S.

Schochetman, Irwin

$1 \mathrm{P}$

$2 \mathrm{P}$

22Exx Lie groups (For the topology

of Lie groups and homogeneous

spaces, see 57Exx, 57Fxx; for the analysis thereof, see $43 \mathrm{~A} 80,43 \mathrm{~A} 85$,

43A90.

22E05 Local Lie groups [See also $34-\mathrm{XX}, 35-\mathrm{XX}, 58 \mathrm{H05}$.]

22 E10 General properties and structure of complex Lie groups

Conlon, Lawrence

22E15 General properties and structure of real Lie groups

$$
\begin{aligned}
& \text { Goto, Morikuni } \\
& \text { Hanson, T. H. McH. } \\
& \text { Lee, Dong Hoon }
\end{aligned}
$$

22E20 General properties and structure of other Lie groups

22E25 Nilpotent and solvable Lie groups

22E30 Analysis on real and complex Lie groups [See also 33A75, 43-XX.]

$$
\text { Baggett, Larry }
$$

22E35 Analysis on p-adic Lie groups [See also 12A 85.]

$$
\text { Silberger, Allan J. }
$$

22E40 Discrete subgroups of Lie groups [See also $32 \mathrm{Nxx}$.]

$$
\begin{aligned}
& \text { Wang, S. P. } \\
& \text { Wang, S. P. } \\
& \text { Wang, S. P. } \\
& \text { Mumford, David } \\
& \text { Wolf, Joseph A. } \\
& \text { Lee, Dong Hoon }
\end{aligned}
$$$$
\begin{aligned}
& 1 P \\
& 2 P \\
& 3 P \\
& 1 P \\
& 2 P \\
& 2 P
\end{aligned}
$$

22E43 Structure and representation of the Lorentz group

22E45 Representations of Lie and linear algebraic groups: analytic methods [For the purely algebraic theory, see 20G05.]

$$
\text { Wang, S. P. }
$$

22E50 Representations of Lie and linear algebraic groups over local fields: analytic methods [For the purely algebraic theory, see 20G05.]
22E55 Representations of Lie and linear algebraic groups over global fields and adèle rings: analytic methods [For the purely algebraic theory, see 20G05.]

22E60 Lie algebras of Lie groups [For the algebraic theory of Lie algebras, see 17Bxx.]

22E65 Infinite dimensional Lie groups and their Lie algebras [See also 17B65, 58H05.]

22E70 Applications of Lie groups to physics; explicit representations [See also $81-\mathrm{XX}$.]

22E99 None of the above, but in this section

Hanson, T. H. McH. $1 \mathrm{~S}$

\section{6-XX REAL FUNCTIONS}

\{See also 54C30.

26-00 Difficult to classify at the second level (must also be assigned at least one other classification number in this section)

26- 01 Elementary exposition (collegiate level)

26-02 Advanced exposition (research surveys, etc.)

26-03 Historical

26-04 Explicit machine computation and programs (not the theory of computation or programming)

26A03 Foundations: limits and generalizations, elementary topology of the line

$$
\text { Aberth, Oliver }
$$

26A06 One-variable calculus

26A09 Elementary functions

26A12 Rate of growth of functions, orders of infinity, slowly increasing functions [See also 26A48.]

Andersen, G. R.

26A15 Continuity and related questions (modulus of continuity, semicontinuity, discontinuities, etc.) [For properties determined by Fourier coefficients, see 42A16; for those determined by approximation properties, see 41A25.]

$$
\begin{aligned}
& \text { Kaczynski, T. J. } \\
& \text { Huneke, John Philip } \\
& \text { Brown, Jack B. } \\
& \text { Peek, Darwin E. }
\end{aligned}
$$

26A16 Lipschitz (Hölder) classes 
Kaufman, Robert $P$.

$1 \mathrm{P}$

26A18 Iteration [See also 39A20.]

Huneke, John Philip

Franks, R. L.

Marzec, R. P.

26A21 Classification of real functions;

Baire classification of sets and functions [See also 04A15, 28A05, 54C50.]

Erdös, Paul
Stone, A. H.
Lewis, Paul W.
Dinculeanu, Nicolae
Darst, Richard B.
Peek, Darwin E.

26A24 Differentiation (functions of one variable): general theory, generalized derivatives, mean-value theorems [See also $28 \mathrm{~A} 15$.]

Baisnab, A. P.

Kaufman, Robert $P$.

26A27 Nondifferentiability

(nondifferentiable functions, points of nondifferentiability), discontinuous derivatives

\section{McCarty, G. S., Jr. \\ Kronheimer, E. H.}

Geroch, Robert P.

26A30 Singular functions, Cantor functions, functions with other special properties

$$
\begin{aligned}
& \text { Herod, J. V. } \\
& \text { Huneke, John Philip } \\
& \text { Kaufman, Robert P. }
\end{aligned}
$$

Peek, Darwin E.

26A33 Fractional derivatives and integrals

Kaufman, Robert $P$

26A36 Antidifferentiation

26A39 Denjoy and Perron integrals, other special integrals

Manougian, Manoug N.
Helton, Burrell, W.
Helton, Burrell, W.
Chatfield, J. A.
Davis, Wilbor P.

26A42 Integrals of Riemann, Stieltjes and Lebesgue type [See also 28-XX.]

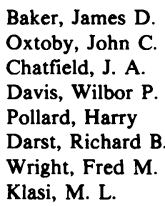

26A45 Functions of bounded variation, generalizations

Chatfield, J. A.

Davis, Wilbor P.

Kaufman, Robert P.

26A48 Monotonic functions, generalizations

Baisnab, A. P.

26A51 Convexity, generalizations

Gollwitzer, H. E.

26A54 Several variables: continuity and differentiation questions

Kaczynski, T. J.

IP

26A57 Several variables: implicit function theorems, Jacobians, transformations with several variables

McShane, Edward James Warfield, R. B., Jr.

26A60 Calculus of vector functions

26A63 Integration: length, area, volume

26A66 Integral formulas (Stokes, Gauss, Green, etc.)

26A69 Special properties of functions of several variables, Hölder conditions, etc.

26A72 Superposition of functions

26A75 Polynomials (analytic properties, inequalities, etc.)

26A78 Polynomials (location of zeros) [See also 12D10, 30A08, 65H05.]

Das, Minaketan

26A81 Rational functions

26A82 Inequalities for trigonometric functions and polynomials

26A84 Inequalities involving derivatives and differential and integral operators

$\begin{array}{ll}\text { Das, K. M. } & \text { 1P } \\ \text { Helton, Burrell, W. } & \text { 1P } \\ \text { Gollwitzer, H. E. } & \text { IP } \\ \text { Pollard, Harry } & \text { 2P } \\ \text { Darst, Richard B. } & \text { 2P }\end{array}$

26A86 Inequalities for sums, series and integrals

Menon, K. V.

Ornstein, Donald S

Herod, J. V.

Darst, Richard B.

Pollard, Harry

Daykin, David E.

Wright, Fred $M$

Klasi, M. L.

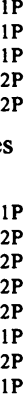

26A87 Other analytical inequalities

26A90 Real-analytic functions

26A93 $\mathrm{C}^{\infty}$-functions, quasi-analytic functions 
Darst, Richard B.

Hughes, Robert B.

Darst, Richard B.

26A96 Calculus of functions on infinite-dimensional spaces

Einhorn, Sheldon J.

Goodman, Victor

26A98 Nonstandard analysis [See also 02H25.]

\section{8-XX MEASURE AND}

INTEGRATION \{For analysis on manifolds, see $58-\mathrm{XX}$.

$28-00$ Difficult to classify at the second level (must also be assigned at least one other classification number in this section)

28-01 Elementary exposition (collegiate level)

28-02 Advanced exposition (research surveys, etc.)

28-03 Historical

28-04 Explicit machine computation and programs (not the theory of computation or programming)

28A05 Classes of sets (Borel fields, $\sigma$-rings, etc.) measurable sets, Suslin sets, analytic sets [See also 04A15, 26A21, 54H05.]

Gelbaum, Bernard R.
Willmott, R. C.
Stone, A. H.
Erdös, Paul
Neubrunn, Tibor
Comfort, W. W.
Darst, Richard B.
Sharp, Henry S.
Mansfield, Richard
Darst, Richard B.
Hansell, Roger W.
Darst, Richard B.
Peek, Darwin E.

28A10 Real- or complex-valued set functions, contents, measures, outer measures, capacities, etc.

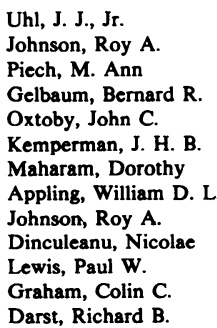

Walsh, B. J.

$1 \mathbf{P}$

28A15 Abstract differentiation theory, differentiation of set functions [See also 26A24.]

$$
\begin{aligned}
& \text { Pfeffer, W. } \\
& \text { Wilbur, W. John } \\
& \text { Uhl, J. J., Jr. }
\end{aligned}
$$

28A20 Measurable and nonmeasurable functions, sequences of measurable functions, modes of convergence

$\begin{array}{ll}\text { Darst, Richard B. } & \text { 4S } \\ \text { Halpern, Benjamin R. } & \text { 2P } \\ \text { Hawley, Douglas } & \text { IS }\end{array}$

28A25 Integration with respect to measures and other set functions

$\begin{array}{ll}\text { Uhl, J. J., Jr. } & \text { 1P } \\ \text { Schmeidler, David } & \text { 1P } \\ \text { Darst, Richard B. } & \text { SP }\end{array}$

28A30 Integration theory via linear functionals (Radon measures, Daniell integrals, etc.)

Foguel, Shaul Reuven

$$
1 \mathbf{P}
$$

28A35 Measures and integrals in product spaces

$\begin{array}{ll}\text { Jamison, Benton } & 2 \mathrm{P} \\ \text { Orey, Steven } & 1 \mathrm{P} \\ \text { Darst, Richard B. } & 5 \mathrm{~S}\end{array}$

28A40 Measures and integrals in function spaces and linear spaces, Weiner measure [See also 46 Gxx.]

Park, Chul
Jamison, Benton
Orey, Steven
Johnson, Gerald W.
Yeh, J.
Jain, N. C.
Kuelbs, J. D.
Johnson, Gerald W.

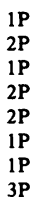

28A45 Vector-valued measures and integrals, integration of vector-valued functions [See also 46G10.]

$$
\begin{aligned}
& \text { Brooks, James K. } \\
& \text { Uhl, J. J., Jr. } \\
& \text { Uhl, J. J., Jr. } \\
& \text { Darst, Richard B. } \\
& \text { Schmeidler, David } \\
& \text { Lewis, Paul W. } \\
& \text { Dinculeanu, Nicolae } \\
& \text { Walsh, B. J. } \\
& \text { Edwards, J. R. } \\
& \text { Wayment, S. G. } \\
& \text { Edwards, J. R. }
\end{aligned}
$$

8A50 Integration and disintegration of measures [For lifting theory, see 46G15.]

28A55 Measures and integrals with values in general ordered systems

28A60 Measures on Boolean rings, 
measure algebras [See also 54H10.]

$$
\text { Darst, Richard B. }
$$

28A65 Measure-preserving transformations, flows (dynamical systems), measure-theoretic ergodic theory [See also 47A35, 54H20, 58Fxx.]

Sentilles, F. Dennis

Ornstein, Donald S.

Chacon, Rafael V.

Goodwyn, Lew Wayne

Akcoglu, M. A

Cunsolo, J.

Simons, F. H.

Hartman, Philip

Sine, R. C.

Chacon, Rafael V.

Schwartzbauer, $T$.

Akcoglu, M. A.

Baxter, J. R.

Jones, Lee

Kuftinec, Velimir

Westman, Joel J.

28A70 Invariant measures, Haar measure [See also 43A05.]

Peterson, H. LeRoy
Coury, John E.
Atalla, Robert E.
Chou, Ching
Rigelhof, Roger
Tserpes, Nicholas
Mukherjea, Arunava
Joffe, A. D.
Chou, Ching
Hawley, Douglas

28 A75 Length, area, volume, other geometric measure theory [See also 26A63, 49F20.]

$$
\begin{aligned}
& \text { Garnett, John } \\
& \text { Kaufman, Robert P. } \\
& \text { Kaufman, Robert P. }
\end{aligned}
$$

30-XX FUNCTIONS OF A COMPLEX VARIABLE (For analysis on manifolds, see 58 $-\mathrm{XX}$. $\}$

30-00 Difficult to classify at the second level (must also be assigned at least one other classification number in this section)

30-01 Elementary exposition (collegiate level)

Dixon, John D.

$1 \mathrm{P}$

30-02 Advanced exposition (research surveys, etc.)

$30-03$ Historical

30-04 Explicit machine computation and programs (not the theory of computation or programming)

30A02 Monogenic properties of complex functions (including polygenic and areolar monogenic functions)

$$
\text { Krajkiewicz, P. }
$$

$30 \mathrm{~A} 04$ Inequalities in the complex domain

$$
\text { Widom, Harold }
$$

30A06 Polynomials

Nassif, $M$.

Shaffer, Dorothy Browne

Beller, E.

Saff, E. B.

Rubinstein, Zalman

Beller, E.

$30 \mathrm{~A} 08$ Zeros of polynomials, rational functions, and other analytic functions (e.g. zeros of functions with bounded Dirichlet integral) [For algebraic theory, see 12D10; for real methods, see 26A78.]

$\begin{array}{ll}\text { Goodman, Adolph W. } & \text { IP } \\ \text { Ratti, Jogindar S. } & \text { 2P } \\ \text { Berndt, Bruce C. } & \text { IP } \\ \text { Caughran, James G. } & \text { IS } \\ \text { Twomey, J. B. } & \text { 2P } \\ \text { Saff, E. B. } & \text { IP } \\ \text { Flaschka, Hermann } & \text { IP } \\ \text { Marden, Morris } & \text { 2S }\end{array}$

30A10 Power series (including lacunary series)

30A12 Boundary behavior of power series, overconvergence

30A14 Analytic continuation

Bogdanowicz, Witold $\mathbf{M}$.

$1 \mathbf{P}$

$30 A 16$ Dirichlet series and other series expansions, exponential series [See also $40-X X, 41-X X, 42-X X$.]

Ogg, A. P.

Layman, J. W.

$1 P$
$1 P$

30A18 Completeness problems, closure of a system of functions

Chang, John S. M.

$1 \mathrm{P}$

30A20 Functional equations in the complex domain, iteration and composition of analytic functions [See also 34A20, 39A15.]

Brady, Michael M. $1 \mathbf{P}$

30A22 Continued fractions [See also 10A30, 40A15.]

Hag, Kari

$1 \mathrm{P}$

30A24 Conformal mappings of special domains

30A26 Covering theorems in conformal 
mapping theory

30A28 Numerical methods in conformal mapping theory

30A30 General theory of conformal mappings

Rosenthal, Paul
Zalcman, Lawrence
Jenkins, James A.
Arsove, Maynard G.
Parnes, Milton N.
Başgöze, Türkân
Warren, Hugh E.
Geveci, Tunc

30A31 Kernel functions and applications

Rosenthal, Paul

Suffridge, Ted J.

Goldstein, Myron

$1 \mathrm{P}$

2S

30A32 Special classes of univalent and multivalent functions (star-like, convex, bounded rotation, etc.)

Livingston, Albert E.
Hummel, James A.
Twomey, J. B.
Bernardi, S. D.
Miller, James
Başgöze, Türkân
Wright, D. J.
Merkes, E. P.
Hengartner, Walter
Al-Amiri, Hassoon S.
Noonan, James W.
Liu, Ming-chit
Miller, Sanford S.
Livingston, Arthur E.
Miller, Sanford S.

Zlotkiewicz, Eligiusz

$1 \mathrm{P}$
$1 \mathrm{P}$
$1 \mathrm{P}$
$1 \mathrm{P}$
$1 \mathrm{P}$
$1 \mathrm{P}$
$3 \mathrm{~S}$
$1 \mathrm{~S}$

30A34 Coefficient problems for univalent and multivalent functions

Livingston, Albert E.
Hummel, James A.
Causey, W. M.
Duren, Peter L.
Hengartner, Walter
Noonan, James W.
Miller, Sanford S.
Miller, Sanford S.

30A36 General theory of univalent and multivalent functions

Robertson, M. S.

30A38 Extremal problems, variational methods

30A40 Extremal problems, other methods

Beller, E.

2S

30A42 Maximum principle; Schwarz' Lemma, Lindelöf principle, analogues and generalizations

Williams, D. L.

1P
Sinclair, Allan $\mathbf{M}$.

Robertson, M. S.

$2 S$
$1 P$

30A44 Capacity and harmonic measure in the complex plane [See also 31A15.]

Garnett, John

$1 \mathrm{P}$

30A46 Compact Riemann surfaces and uniformization [See also $14 \mathrm{H} 15,32 \mathrm{G} 15$.]

Kra, Irwin

Wong, C. K.

Wong, C. $\mathrm{K}$

Rao, K. V. R.

Metzger, Thomas A

MacLachlan, C.

$1 \mathrm{~S}$

2P

1S

1P

30A48 Classification theory of Riemann surfaces

Accola, Robert D. M. 1 P

Accola, Robert D. M.

30A50 Ideal boundary theory

30A52 Differentials on Riemann surfaces

Farkas, Hershel $\mathbf{M}$.

$1 P$

30A58 Discontinuous groups and automorphic functions [See also 10Dxx, 20H10, 32Nxx.]

$\begin{array}{ll}\text { Accola, Robert D. M. } & \text { 1P } \\ \text { Kra, Irwin } & \text { 1P } \\ \text { Wong, C. K. } & 1 \mathrm{P} \\ \text { Wong, C. K. } & \text { 2P } \\ \text { Rao, K. V. R. } & 1 \mathrm{P} \\ \text { Metzger, Thomas A. } & 1 \mathrm{P} \\ \text { MacLachlan, C. } & 1 \mathrm{P}\end{array}$

30A60 Quasiconformal mappings and functions

$\begin{array}{ll}\text { Zalcman, Lawrence } & \text { 1S }\end{array}$

30A62 Representations of entire functions by series and integrals

Bose, Anil Kumar

Marden, Morris

30A64 Special classes of entire functions and growth estimates

$\begin{array}{ll}\text { Bose, Anil Kumar } & \text { IP } \\ \text { Pugh, W. J. } & \text { IP } \\ \text { Marden, Morris } & \text { 2P }\end{array}$

30A66 Entire functions, general theory

Bose, Anil Kumar

Yang, Chung-chun

$1 \mathrm{~S}$

30A68 Meromorphic functions, general theory

Miles, Joseph

Cullen, Michael R.

Yang, Chung-chun

$1 \mathrm{P}$

$30 A 70$ Distribution of values,

Nevanlinna theory

Bank, Steven

$1 \mathbf{P}$ 
Krajkiewicz, P.

Yang, Chung-chun

30A72 Cluster sets, prime ends, boundary behavior

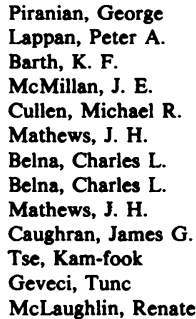

30A74 Normal functions, normal families

$$
\text { Tse, Kam-fook }
$$

30A76 Blaschke products, bounded characteristic, bounded functions, functions with positive real part

Barth, K. F.

Schneider, W. J.

Cullen, Michael R.

Caughran, James G

Robertson, M. S.

Tse, Kam-fook

McLaughlin, Renate

$30 \mathrm{~A} 78 \mathrm{H}^{\mathrm{p}}$, quasianalytic and other classes of functions

Hedlund, James H.
Duren, Peter L.
Schubert, C. F.
Yamashita, Shinji
Caveny, D. J.
Novinger, W. P.
Taylor, Gerald D.
Salehi, Habib
Başgöze, Türkân
Yabuta, Kôzô
Scheinberg, Stephen
Miller, Sanford S.

30A80 Moment problems, interpolation problems

$$
\begin{aligned}
& \text { Caveny, D. J. } \\
& \text { Novinger, W. P. } \\
& \text { Tse, Kam-fook }
\end{aligned}
$$

30A82 Approximation in the complex domain

Layman, J. W.

Ginsberg, Jonathan I.

Nassif, $M$.

O'Hara, P. J.

Saff, E. B.

Rubinstein, Zalman

Saff, E. B.

Davie, Alexander $M$

$\emptyset$ ksendal, B. K.

Chang, John S. M
30A84 Asymptotic representations in the complex domain

30A86 Integration, integrals of Cauchy type, integral representations of analytic functions [See also 45Exx.]

Kulshreshtha, S. K.

Arora, Kasturi L.

$$
1 \mathrm{P}
$$

30A88 Boundary value problems

$\begin{array}{ll}\text { Schneider, W. J. } & \text { is } \\ \text { Barth, K. F. } & \text { IS }\end{array}$

Goldstein, Myron

30A90 Topological function theory

$\begin{array}{ll}\text { Verhey, Roger F. } & \text { 1P } \\ \text { Marx, Morris L. } & 2 P \\ \text { Mathews, J. H. } & 2 P\end{array}$

30A91 Nonstandard function theory [See also 02H25.]

30A92 Generalized analytic functions

Krishnasastry, M. S. R.

30A93 Pseudo-analytic functions

30A94 p-analytic functions

Bosch, W.

Krajkiewicz, P.

IP

30A95 Discrete analytic functions

30A96 Other generalizations of analytic functions (including abstract-valued functions)

$\begin{array}{ll}\text { Bogdanowicz, Witold M. } & \text { 1P } \\ \text { Krajkiewicz, P. } & 2 \mathrm{P} \\ \text { Krishnasastry, M. S. R. } & \text { 1S } \\ \text { Salehi, Habib } & \text { 2P }\end{array}$

30A97 Functions of hypercomplex variables and generalized variables

30A98 Spaces and algebras of analytic functions [See also 46J15.]

$\begin{array}{ll}\text { Cima, Joseph A. } & \text { 1P } \\ \text { Eifler, Larry } & \text { 1S } \\ \text { Wilken, Donald R. } & \text { 2P } \\ \text { Hoffmann, Laurence D. } & \text { 1P } \\ \text { Boehme, T. K. } & 2 \text { P } \\ \text { Carpenter, R. L. } & 2 S\end{array}$

\section{1-XX POTENTIAL}

THEORY \{For probabilistic potential theory, see 60J45. \}

$31-00$ Difficult to classify at the second level (must also be assigned at least one other classification number in this section)

$31-01$ Elementary exposition (collegiate level)

31-02 Advanced exposition (research 
surveys, etc.)

31-03 Historical

31-04 Explicit machine computation and programs (not the theory of computation or programming)

\section{Axx Two-dimensional theory}

31A05 Harmonic, subharmonic, superharmonic functions

$$
\text { Goldstein, Myron }
$$

31 A10 Integral representations

$31 \mathrm{~A} 15$ Potentials and capacity, harmonic measure, extremal length

31A20 Boundary behavior (Fatou type theorems, etc.)

$$
\begin{aligned}
& \text { Weiss, Max L. } \\
& \text { Boehme, T. K. } \\
& \text { Boehme, T. K. } \\
& \text { Geveci, Tunc }
\end{aligned}
$$

Meek, James L.

$1 \mathbf{P}$
$1 \mathbf{P}$
$2 \mathbf{P}$
$1 \mathrm{~S}$
$1 \mathrm{P}$

31A25 Boundary value and inverse problems

Goldstein, Myron

31A30 Biharmonic, polyharmonic functions and equations, Poisson's equation

31 A35 Connections with differential equations

31A99 None of the above, but in this section

\section{Bxx Higher dimensional theory}

31B05 Harmonic, subharmonic, superharmonic functions

$$
\begin{aligned}
& \text { Chow, Kwang-nan } \\
& \text { Glasner, Moses } \\
& \text { Sibuya, Yasutaka }
\end{aligned}
$$

31B10 Integral representations

31B15 Potentials and capacities, extremal length

31 B20 Boundary value and inverse problems

31B25 Boundary behavior

31B30 Biharmonic and polyharmonic equations and functions

31B35 Connections with differential equations

$$
\begin{aligned}
& \text { Chow, Kwang-nan } \\
& \text { Glasner, Moses } \\
& \text { Sibuya, Yasutaka }
\end{aligned}
$$

$1 P$
$1 P$

31 B99 None of the above, but in this section

\section{Cxx Other generalizations}

31C05 Harmonic, subharmonic, superharmonic functions

$\begin{array}{ll}\text { Stanford, David P. } & \text { IP } \\ \text { Meek, James L. } & \text { IP }\end{array}$

$31 \mathrm{C} 10$ Pluriharmonic and plurisubharmonic functions [See also 32F05.]

$31 \mathrm{C} 15$ Potentials and capacities

Hebert, D. J., Jr. 1P

$31 \mathrm{C} 20$ Discrete potential theory and numerical methods

Dubuc, Serge

31C25 Dirichlet spaces

Glasner, Moses

Chow, Kwang-nan

31 C99 None of the above, but in this section

31D05 Axiomatic potential theory

$$
\begin{aligned}
& \text { Glasner, Moses } \\
& \text { Chow, Kwang-nan }
\end{aligned}
$$

32-XX SEVERAL COMPLEX VARIABLES AND ANALYTIC

\section{SPACES}

32-00 Difficult to classify at the second level (must also be assigned at least one other classification number in this section)

32-01 Elementary exposition (collegiate level)

32-02 Advanced exposition (research surveys, etc.)

32-03 Historical

32-04 Explicit machine computation and programs (not the theory of computation or programming)

32Axx Holomorphic functions of several complex variables

32A05 Power series, series of functions

32A07 Special domains (Reinhardt, Hartogs, tube domains, etc.)

$$
\text { Kraut, Edgar A. }
$$

32A10 Holomorphic functions

$\begin{array}{ll}\text { Greenfield, Stephen J. } & \text { 1P } \\ \text { Williams, G. Kenneth } & \text { IP } \\ \text { Busenberg, Stavros } & \text { IP }\end{array}$

32A15 Entire functions 
32A17 Normal families

32A20 Meromorphic functions

32A25 Integral representation and residue theory

Busenberg, Stavros

32A30 Other generalizations of function theory of one complex variable [For functions of several hypercomplex variables, see 30A97.]

32A99 None of the above, but in this section

Yabuta, Kôzô

32Bxx Local analytic geometry \{See also $13-\mathrm{XX}$ and $14-\mathrm{XX}$.

32B05 Analytic algebras and generalizations, preparation theorems

Allan, G. R.

1S

32B10 Germs of analytic sets

32B15 Analytic subsets of affine space

32B20 Semi-analytic sets

32B99 None of the above, but in this section

\section{Cxx General theory of analytic spaces}

32C05 Real-analytic manifolds, real-analytic spaces

Greenfield, Stephen J.
Hunt, L. R.

32C10 Complex manifolds [For almost complex manifolds, see 53C15.]

Barth, Theodore J.
Stoll, Wilhelm

Chen, Su-shing

32C15 Complex spaces

Siu, Yum-tong

Fernholz, E. R.

32C20 Normal analytic spaces

32 C25 Analytic subsets and submanifolds

Emerson, William R.

Thie, Paul R.

Stoll, Wilhelm

Kiernan, Peter

Thie, Paul R.

Shiffman, Bernard

$32 \mathrm{C} 30$ Integration on analytic sets and spaces

Emerson, William R.

Thie, Paul R.
32C35 Analytic sheaves and cohomology groups [See also $18 \mathrm{~F} 20,55 \mathrm{~B} 30$.]

Kripke, Bernard R.

$1 \mathrm{P}$

$32 \mathrm{C} 40$ Singularities

$32 \mathrm{C} 45$ Modifications, resolution of singularities [See also 14E15.]

32 C99 None of the above, but in this section

32Dxx Analytic continuation

32D05 Domains of holomorphy

32D10 Envelopes of holomorphy

Weinstock, Barnet M.

Schaeffer, David G.

$1 \mathbf{P}$
$2 \mathbf{P}$

Hunt, L. R.

32D15 Continuation of analytic objects

Fitzgerald, Carl H.

Weinstock, Barnet $\mathbf{M}$.

$1 \mathrm{P}$

32D20 Removable singularities

32D99 None of the above, but in this section

\section{Exx Holomorphic convexity}

32E05 Holomorphically convex complex spaces, reduction theory

$\begin{array}{ll}\text { Fernholz, E. R. } & \text { IP } \\ \text { Greenfield, Stephen J. } & \text { iS }\end{array}$

32E10 Stein spaces, Stein manifolds

Kalmbach, Gudrun $\quad$ 1P

Hunt, L. R.

$1 \mathrm{~S}$

32E15 Runge pairs

32E20 Polynomial convexity

32E25 Algebras of holomorphic functions

32 E30 Holomorphic and polynomial approximation

Weinstock, Barnet $M$.

2P

32 E99 None of the above, but in this section

\section{Fxx Geometric convexity}

32F05 Plurisubharmonic functions and generalizations [See also $31 \mathrm{C} 10$.]

32F10 q-convexity, q-concavity

32F15 Pseudoconvex domains

Weinstock, Barnet $\mathbf{M}$.

$1 P$

32F99 None of the above, but in this section [For the theory of the

-Neumann problem on pseudoconvex domains, see $35 \mathrm{~N} 15$.] 
32Gxx Deformations of analytic structures

32G05 Deformations of complex structures [See also 13D10, 16A58, 58H05.]

32G10 Deformations of submanifolds and subspaces

32G13 Analytic moduli problems [For algebraic moduli problems, see 14D20.]

32G15 Moduli of Riemann surfaces, Teichmüller theory [See also 30A46.]

32G20 Period matrices

32G99 None of the above, but in this section

32Hxx Analytic mappings

32H05 Representative domains

32H10 Bergman kernel function

Fitzgerald, Carl $\mathrm{H}$.

$1 \mathrm{P}$

$32 \mathrm{H} 15$ Invariant metrics and pseudodistances

Kiernan, Peter

$1 \mathrm{P}$

32H20 Hyperbolic complex manifolds

Kiernan, Peter 2P

32H 25 Picard type theorems and generalizations

$32 \mathrm{H} 99$ None of the above, but in this section

32Jxx Compact analytic spaces Riemann surfaces, see $14 \mathrm{Hxx}$, 30A46.

32J05 Compactification

32J10 Algebraic dependence theorems

32J15 Compact surfaces [See also $14 \mathrm{Jxx}$.

$32 \mathrm{~J} 20$ Algebraicity criteria

32J25 Transcendental methods of algebraic geometry [See also 14C30.]

32J99 None of the above, but in this section

32Kxx Generalizations of analytic spaces

32K05 Banach analytic spaces [See also 58Bxx.]

32K10 Nonarchimedean analytic spaces
32K15 Differentiable functions on analytic spaces, differentiable spaces [See also 58C25.]

$32 \mathrm{~K} 99$ None of the above, but in this section

32Lxx Holomorphic fiber spaces \{See also 55Fxx.

32L05 Holomorphic fiber bundles and generalizations

32L10 Sheaves and cohomology of sections of holomorphic vector bundles [See also 18F20, 55B30.]

Stoll, Wilhelm

2P

32L99 None of the above, but in this section

Seeley, Robert $T$.

$1 \mathbf{P}$

32Mxx Complex spaces with a group of automorphisms

32M05 Complex Lie groups, automorphism groups of complex spaces [See also 22E10.]

Chen, Su-shing

$1 \mathbf{P}$

32M10 Homogeneous complex manifolds [See also 57F15.]

32M15 Hermitian symmetric spaces, bounded symmetric domains [See also 22E10, 22E40, 53C35, 57F15.] Wolf, Joseph A. 3P $32 \mathrm{M} 99$ None of the above, but in this section

32Nxx Automorphic functions \{See also 10Dxx, 20H10, 22E40, 30A58. 32N05 General theory of automorphic functions of several complex variables

$32 \mathrm{~N} 10$ Automorphic forms

32N15 Automorphic functions in symmetric domains

Resnikoff, H. L.
Mumford, David

32 N99 None of the above, but in this section

Hall, Michael H. Mumford, David

$1 \mathrm{P}$

33-XX SPECIAL

FUNCTIONS $\{33-\mathrm{XX}$ deals with the properties of functions as 
functions. General systems of orthogonal functions are treated in 42A52. Expansions in orthogonal functions are treated in 42A56. 33-00 Difficult to classify at the second level (must also be assigned at least one other classification number in this section)

33-01 Elementary exposition (collegiate level)

33-02 Advanced exposition (research surveys, etc.)

33-03 Historical

33-04 Explicit machine computation and programs (not the theory of computation or programming)

33A10 Exponential and trigonometric functions

33A15 Gamma and beta functions

Arora, Kasturi L.

Kulshreshtha, S. K.

33A20 Error function, probability integral

33A25 Elliptic functions and integrals Ogg, A. P.

33A30 Hypergeometric functions of one and several variables, generalizations

Brown, James Ward

Andrews, George E.

Goldberg, J. L.

Brown, J. W.

Kulshreshtha, S. K.

Arora, Kasturi L.

Lahiri, Maya

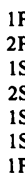

33A35 E-functions, G-functions, etc.

Arora, Kasturi $\mathbf{L}$.

Kulshreshtha, S. K.

$1 \mathrm{P}$

33A40 Cylindrical functions, Bessel functions

33A45 Spherical functions (Legendre polynomials and functions, spherical harmonics), ultraspherical polynomials

Agrawal, Bhagwan Das

Khanna, I. K.

Nath, B.

33A50 Gegenbauer functions Nath, B. $1 \mathrm{P}$

33A55 Lamé, Mathieu, spheroidal wave functions

33A60 Other wave functions

33A65 Orthogonal special functions and polynomials (Cebyšev, Hermite, Jacobi,

Laguerre, etc.)

$\begin{array}{ll}\text { Brown, J. W. } & \text { 1P } \\ \text { Khanna, I. K. } & \text { 1P } \\ \text { Agrawal, Bhagwan Das } & \text { IP } \\ \text { Muckenhoupt, Benjamin } & \text { 1P } \\ \text { Srivastava, H. M. } & \text { 1P } \\ \text { Wilson, M. Wayne } & \text { 1S } \\ \text { Muckenhoupt, Benjamin } & \text { 2P } \\ \text { Zeitlin, David } & \text { 1P } \\ \text { Goldberg, J. L. } & \text { 1P } \\ \text { Brown, J. W. } & 2 \text { P } \\ \text { Lahiri, Maya } & \text { 1P } \\ \text { Das, Minaketan } & 1 P \\ \text { Nath, B. } & \text { 1P } \\ \text { Brenner, Joel Lee } & \text { 2P }\end{array}$

33A70 Other special functions

Carlitz, Leonard

Zeitlin, David

Nath, B.

$1 P$
$1 P$
$1 P$
$1 P$
$1 P$
$1 S$
$2 P$
$1 P$
$1 P$
$2 P$
$1 P$
$1 P$
$1 P$
$2 P$

33A75 Special functions and Lie groups

\section{4-XX ORDINARY} DIFFERENTIAL EQUATIONS

34-00 Difficult to classify at the second level (must also be assigned at least one other classification number in this section)

34-01 Elementary exposition (collegiate level)

34-02 Advanced exposition (research surveys, etc.)

34-03 Historical

34-04 Explicit machine computation and programs (not the theory of computation or programming)

\section{Axx General theory}

34A05 Solutions in closed form, integration by quadratures, reduction of differential equations

$\begin{array}{ll}\text { Kreith, Kurt } & \text { 3P } \\ \text { Benson, Donald C. } & \text { 1P } \\ \text { Reid, James L. } & \text { 1P } \\ \text { Gottlieb, David } & \text { IP } \\ \text { Breuer, Shlomo } & \text { 1P } \\ \text { Zettl, Anton } & \text { 3P }\end{array}$

34A10 Initial value problems: general existence and uniqueness theorems; continuous dependence of solutions on parameters, initial conditions and boundary conditions

$\begin{array}{ll}\text { Wend, David V. V. } & \text { 1P } \\ \text { Roberson, Fred A. } & \text { 1P } \\ \text { Gray, William J. } & \text { 1P } \\ \text { Manougian, Manoug N. } & \text { 2P } \\ \text { Martin, Robert H., Jr. } & \text { 2P }\end{array}$


Ebin, David G.

Metcalf, Frederic T

Bownds, John M.

Grimm, L. J.

Hermes, Henry

Wright, Fred M.

Klasi, M. L.

34A15 Initial value problems: continuation of solutions

Burton, T. A.

Grimmer, R. C.

34A20 Differential equations in the complex domain [See also 30A70.]

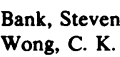

Wong, Pui-kei

34A25 Analytical theory: series, transformations, transforms, operational calculus, etc. [See also 44-XX, 47E05.]

34A30 Linear equations and systems

34A35 Differential equations of infinite order

Al-Salam, Waleed A.

Dickey, R. W.

Ebin, David G.

34A40 Differential inequalities

Murdeshwar, M. G.
Deo, S. G.
Lovelady, David Lowell
Gunderson, R. W.
Fraker, Ross
Bebernes, J. W.
Fraker, Ross
Deo, S. G.

34A45 Theoretical approximation of solutions

Stein, F. Max

Henry, Myron S.

34A50 Numerical approximation of solutions [For numerical analysis, see 65Lxx.]

34A99 None of the above, but in this section

\section{Bxx Boundary value problems} 34B05 Linear equations

$\begin{array}{ll}\text { Zettl, Anton } & \text { 1P } \\ \text { Hochstadt, Harry } & \text { 1S } \\ \text { Bogar, Gary A. } & \text { 1P } \\ \text { Hughes, David K. } & \text { 1P } \\ \text { Zettl, Anton } & \text { 2P }\end{array}$

34B10 Multipoint boundary value problems

Bryan, Robert Neff

34B15 Nonlinear boundary value problems

\author{
Schrader, Keith W. \\ Waltman, Paul E \\ George, John H. \\ Sutton, W. G. \\ Fraker, Ross \\ Bebernes, J. W. \\ Fraker, Ross
}

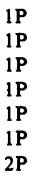

34B20 Weyl theory and its generalizations

34B25 Spectral theory, Sturm-Liouville, and scattering theory; eigenfunctions, eigenvalues, and expansions [See also 42-XX, 43-XX, 47Axx, 47Bxx, 47E05.]

\section{Eastham, M. S. P. \\ Banks, Dallas $O$. \\ Kauffman, Robert M.}

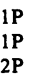

34B30 Special equations (Mathieu, Hill, Bessel, etc.) [See also 33-XX.]

34B99 None of the above, but in this section

\section{Cxx Qualitative theory}

34C05 Limit cycles, singular points

$34 \mathrm{C} 10$ Zeros, disconjugacy, growth, boundedness, oscillation and comparison of solutions

Sherman, Thomas L.
Fink, A. M.
St. Mary, Donald F.
Hochstadt, Harry
Kim, Woo Jong
Peterson, Allan C.
Hartman, Philip
Hethcote, Herbert W.
Gustafson, G. B.
Wend, David V. V.
Ryder, Gerald H.
Kreith, Kurt
Galbraith, A. S.
Utz, W. Roy
Kim, Woo Jong
Etgen, Garret J.
Leighton, Waiter
Ke, William Oo Kian
Schneider, Leo J.
Kim, Woo Jong
Etgen, Garret J.
Scott, J. B.
Breuer, Shlomo
Wong, Pui-kei

$34 \mathrm{C} 15$ Nonlinear oscillations

Heidel, Jon W. Dickey, R. W.

Bobisud, Larry E.

Swanson, C. A

Gollwitzer, H. E.

Onose, Hiroshi

Burton, T. A.

Grimmer, R. C

Hammett, Michasl E

Kartsatos, A. G.
$P$
$P$
$P$ 
Cronin-Scanlon, Jane

Lasota, Andrzej

Bernfeld, Stephen R.

$1 \mathbf{P}$
$1 \mathbf{P}$
$2 \mathbf{P}$

$34 \mathrm{C} 20$ Transformation and reduction of equations and systems

$$
\text { Proctor, Thomas Gilmer }
$$

\section{$1 \mathbf{P}$}

34C25 Periodic and almost periodic solutions

$$
\begin{aligned}
& \text { Yorke, James A. } \\
& \text { Proctor, Thomas Gilmer } \\
& \text { Schmitt, Klaus } \\
& \text { Fink, A. M. } \\
& \text { Cronin-Scanlon, Jane }
\end{aligned}
$$

$2 \mathrm{P}$
$2 \mathrm{P}$
$1 \mathrm{P}$
$2 \mathrm{P}$
$1 \mathrm{P}$

34C30 Manifolds of solutions, averaging method [See also 47H10, 58Fxx.]

34C35 Dynamical systems [See also 58Fxx, 70-XX.]

$$
\begin{aligned}
& \text { Gordon, William B. } \\
& \text { Weinstein, Alan D. } \\
& \text { Marsden, Jerrold E. } \\
& \text { Ebin, David G. } \\
& \text { Hájek, Otomar } \\
& \text { Palis, J. }
\end{aligned}
$$

$34 \mathrm{C40}$ Equations and systems on manifolds [See also 58Fxx, 58Gxx.] $34 C 99$ None of the above, but in this section

\section{Dxx Stability theory \{ See also} 58F10, 93Dxx.

34D05 Asymptotic properties, characteristic exponents

Katz, I. Norman

Zettl, Anton

Mayes, Vivienne

Zaidman, Samue

Zettl, Anton

Lakshmikantham, V.

Ladas, Gerasimos

Leela, $\mathbf{S}$.

Hallam, Thomas G.

Hammett, Michael E.

Bernfeld, Stephen R.

Lasota, Andrzej

34D10 Perturbations

Yorke, James A.

Strauss, Aaron

34D15 Singular perturbations

34D20 Ljapunov stability

Zaidman, Samuel

Gunderson, R. W.

Deo, S. G.

34D25 Popov-type stability

34D30 Structural stability and analogous concepts [See also 58Fxx.]
34D35 Stability of manifolds of solutions 34D99 None of the above, but in this section

\section{Exx Asymptotic theory}

34E05 Asymptotic expansions

Hallam, Thomas Muckenhoupt, Benjamin $\quad$ 2P

34E10 Perturbations

$\begin{array}{ll}\text { Ladas, Gerasimos } & 1 \mathrm{P} \\ \text { Lakshmikantham, V. } & 1 \mathrm{P} \\ \text { Leela, S. } & 1 \mathrm{P}\end{array}$

34E15 Singular perturbations, general theory

Chang, $\mathrm{K}$. W.

$1 \mathbf{P}$

34E20 Singular perturbations, turning point theory, WKB-methods

34E99 None of the above, but in this section

34F05 Equations and systems with randomness \{See also 60K05, 93Exx. $\}$

34G05 Differential equations in Banach and other abstract spaces \{See also 47Bxx.

$\begin{array}{ll}\text { Zaidman, Samuel } & \text { 1P } \\ \text { Goldstein, Jerome A. } & \text { 2P } \\ \text { Zaidman, Samuel } & \text { 2P } \\ \text { Martin, Robert H., Jr. } & \text { 2P } \\ \text { Lovelady, David Lowell } & \text { IP }\end{array}$

34H05 Control problems \{See also 49-XX, 93-XX.

Klasi, M. L.

Wright, Fred $M$

$2 \mathrm{~S}$

34Jxx Functional differential equations

34J05 General theory

$\begin{array}{ll}\text { Benson, Donald C. } & \text { 1P } \\ \text { Kreith, Kurt } & \text { 3P }\end{array}$

34J10 Differential-difference equations

Hughes, David K.

$1 P$

34J99 None of the above, but in this section

34Kxx Functional-differential equations with retarded arguments, functional-differential equations with 


\section{deviating arguments}

34K05 General theory

Yorke, James A.

Grimm, L. J.

34K10 Boundary value problems

34K15 Qualitative theory

Schmitt, Klaus

34K20 Stability theory

Abramowich, John

Izé, Antonio F.

Hale, Jack $\mathrm{K}$.

34K25 Asymptotic theory

$34 \mathrm{~K} 99$ None of the above, but in this section

\section{5-XX PARTIAL}

\section{DIFFERENTIAL EQUATIONS}

35-00 Difficult to classify at the second level (must also be assigned at least one other classification number in this section)

35-01 Elementary exposition (collegiate level)

35-02 Advanced exposition (research surveys, etc.)

35-03 Historical

35-04 Explicit machine computation and programs (not the theory of computation or programming)

\section{Axx General theory}

35A05 General existence and uniqueness theorems

$$
\begin{aligned}
& \text { Rupp, Russell D., Jr. } \\
& \text { Sigillito, Vincent G. } \\
& \text { Garabedian, Paul R. } \\
& \text { Diaz, Joaquin B. }
\end{aligned}
$$

35A10 Cauchy-Kowalewski theorems

35A15 Variational methods

35A20 Analytic methods, singularities

Bragg, Louis R.

$2 \mathbf{P}$

35A22 Transform methods

Dettman, John W.

Bragg, Louis $\mathbf{R}$.

$1 \mathrm{P}$

$1 P$

35A25 Other special methods

35A30 Geometric theory, characteristics, transformations

35A35 Theoretical approximation to solutions
35A40 Numerical approximation to solutions [For numerical analysis, see $65 \mathrm{Mxx}, 65 \mathrm{Nxx}, 65 \mathrm{P} 05$.

35A99 None of the above, but in this section

35Bxx Qualitative properties of solutions

35B05 Comparison theorems; oscillation, zeros and growth of solutions

$\begin{array}{ll}\text { Allegretto, Walter } & 1 P \\ \text { Headley, V. B. } & 1 P \\ \text { Swanson, C. A. } & 2 P \\ \text { Allegretto, Walter } & 2 P \\ \text { Kreith, Kurt } & 5 P\end{array}$

35B10 Periodic solutions

35B15 Almost periodic solutions

35B20 Perturbations

35B25 Singular perturbations

Greenlee, W. M.

Greenlee, W. M.

Sibuya, Yasutaka

$1 \mathbf{P}$
$2 \mathbf{P}$
$1 \mathbf{P}$

35B30 Dependence of solutions on initial and boundary data, parameters

Greenlee, W. M.

$1 \mathrm{P}$

35B35 Stability and control boundedness [See also 93-XX.]

35B40 Asymptotic behavior of solutions Goldstein, Jerome A.

2P

35B45 Differential inequalities, a priori estimates

35B99 None of the above, but in this section

35Cxx Representations of solutions

$35 \mathrm{C05}$ Solutions in closed form

35C10 Series solutions, expansion theorems

$$
\text { Bragg, Louis } \mathrm{R} \text {. } 2 \mathrm{P}
$$

$35 \mathrm{C} 15$ Integral representations

Haimo, Deborah Tepper 1P

35C99 None of the above, but in this section

Schaeffer, David G. $\quad 1$ P

\section{Dxx Generalized solutions}

35D05 Existence of generalized solutions 35D10 Regularity of generalized solutions 
35D99 None of the above, but in this section

Sibuya, Yasutaka

$1 \mathrm{~S}$

35Exx Equations and systems with constant coefficients \{ See also

35N05.

35E05 Fundamental solutions

Barros-Neto, Jose 1P

35E10 Convexity properties

Harvey, Reese

Harvey, Charles

$1 \mathrm{P}$

$1 \mathrm{P}$

$35 \mathrm{E} 15$ Initial value problems

35 E99 None of the above, but in this section

Kascic, Michael J., Jr.

$1 \mathrm{P}$

35Fxx General first order equations and systems

35F05 Linear equations and systems, general

Garabedian, Paul R.

$1 \mathrm{P}$

$35 \mathrm{~F} 10$ Initial value problems for linear equations and systems

$35 \mathrm{~F} 15$ Boundary value problems for linear equations and systems

35F20 Nonlinear equations and systems, general

35F25 Initial value problems for nonlinear equations and systems

35F30 Boundary value problems for nonlinear equations and systems

35F99 None of the above, but in this section

\section{Gxx General higher order} equations and systems

35G05 Linear equations and systems, general

35G10 Initial value problems for linear equations and systems

35G15 Boundary value problems for linear equations and systems

$$
\begin{aligned}
& \text { Dettman, John W. } \\
& \text { Bragg, Louis R. } \\
& \text { Barros-Neto, Jose } \\
& \text { Diaz, Joaquin B. }
\end{aligned}
$$

35G20 Nonlinear equations and systems, general

35G25 Initial value problems for nonlinear equations and systems

35G30 Boundary value problems for nonlinear equations and systems

35G99 None of the above, but in this section

\section{H05 Hypoelliptic equations and systems}

35Jxx Elliptic equations and systems

35J05 Laplace's equation, reduced wave equation (Helmholtz), Poisson's equation

Sibuya, Yasutaka IP

35J10 Schrödinger operator [See also 47A40.]

$35 \mathrm{~J} 15$ Second order equations, general

$\begin{array}{ll}\text { Headley, V. B. } & \text { IP } \\ \text { Kreith, Kurt } & \text { 5P }\end{array}$

35 J20 Second order equations, variational methods

$\begin{array}{ll}\text { Chow, Kwang-nan } & \text { 1S } \\ \text { Glasner, Moses } & \text { is }\end{array}$

$35 \mathrm{~J} 25$ Second order equations, boundary value problems

35J30 Higher order equations, general

Schaeffer, David G.

$1 \mathrm{P}$

35J35 Higher order equations,

variational problems

$35 \mathrm{~J} 40$ Higher order equations, boundary value problems

Greenlee, W. M.

35J45 Systems, general

35J50 Systems, variational methods

35J55 Systems, boundary value problems

$35 \mathrm{~J} 60$ Nonlinear equations and systems

Swanson, C. A.

Allegretto, Walter $2 \mathrm{P}$

$35 \mathrm{~J} 65$ Nonlinear boundary value problems for linear equations and systems

$35 \mathrm{~J} 67$ Boundary values of solutions

$35 \mathrm{~J} 70$ Equations and systems of degenerate type

Allegretto, Walter

$1 P$

35J99 None of the above, but in this section

35Kxx Parabolic equations and systems

$35 \mathrm{~K} 05$ Heat equation 
Hughes, Robert B.

Haimo, Deborah Tepper

$35 \mathrm{~K} 10$ Second order equations, general

$35 \mathrm{~K} 15$ Second order equations, initial value problems

Rosencrans, S. I.

35K20 Second order equations, boundary value problems

Rupp, Russell D., Jr.

$35 \mathrm{~K} 25$ Higher order equations, general

$35 \mathrm{~K} 30$ Higher order equations, initial value problems

$35 \mathrm{~K} 35$ Higher order equations, boundary value problems

$35 \mathrm{~K} 40$ Systems, general

$35 \mathrm{~K} 45$ Systems, initial value problems

$35 \mathrm{~K} 50$ Systems, boundary value problems

35K55 Nonlinear equations and systems

35K60 Nonlinear boundary value problems for linear equations and systems

35K99 None of the above, but in this section

35Lxx Hyperbolic equations and systems

35L05 Wave equation

$\begin{array}{ll}\text { Wolfe, Peter } & 1 \mathrm{P} \\ \text { Sigillito, Vincent G. } & 1 \mathrm{P}\end{array}$

35L10 Second order equations, general

$35 \mathrm{~L} 15$ Second order equations, initial value problems

$$
\text { Rosencrans, S. I. }
$$

35L20 Second order equations, boundary value problems

$$
\text { Diaz, Joaquin B. }
$$

35L25 Higher order equations, general

$$
\text { Peyser, Gideon }
$$

35L30 Higher order equations, initial value problems

35L35 Higher order equations, boundary value problems

35L40 First order systems, general

35L45 First order systems, initial value problems

35L50 First order systems, boundary value problems

35L55 Higher order hyperbolic systems
35 L60 Nonlinear equations and systems, general

35L65 Conservation laws [See also 76Axx.]

35L99 None of the above, but in this section

35M05 Equations and systems of mixed type (Tricomi, etc.) \{For degenerate types, elliptic, see $35 \mathrm{Jxx}$, hyperbolic, see $35 \mathrm{Lxx}$, etc.

\section{Nxx Overdetermined systems}

35N05 Constant coefficients

$35 \mathrm{~N} 10$ Variable coefficients, general

35N15 $\bar{\partial}$-Neumann problem and generalizations; formal complexes [See also 58G05.]

35 N99 None of the above, but in this section

\section{Pxx Spectral theory and} eigenvalue problems (See also 47Axx, 47Bxx, 47F05.

35P05 General spectral theory

35P10 Completeness of eigenfunctions, eigenfunction expansions

$$
\text { Seeley, Robert } \mathrm{T} \text {. }
$$

35P15 Estimation of eigenvalues, upper and lower bounds

35P20 Asymptotic distribution of eigenvalues and eigenfunctions

35P25 Scattering theory [See also 47A40.]

35 P99 None of the above, but in this section

35Qxx Special equations and problems \{ See also 35J05, 35K05, 35L05.\}

35Q05 Euler-Poisson-Darboux equation and generalizations

Bragg, Louis R.

2P

35 Q10 Navier-Stokes equation [See also 76D05.]

35 Q15 Riemann-Hilbert problems [See also 30A88, 31 A25.]

35Q99 None of the above, but in this section 
35Rxx Miscellaneous topics \{For equations on manifolds, see $58 \mathrm{Gxx}$; for manifolds of solutions, see 58Bxx; for stochastic PDE's, see 60H15.

35R05 Equations with discontinuous coefficients or data

35R 10 Difference-partial differential equations, equations with time lag

35R15 Equations on function spaces

35R20 Operator equations, general [See also 34G05, 47A50, 47H15.]

35R25 Improperly posed problems

Sigillito, Vincent G.

$1 \mathrm{P}$

$35 \mathrm{R} 30$ Inverse problems (undetermined coefficients, etc.)

35R99 None of the above, but in this section

\section{Sxx Pseudodifferential operators} \{See also 47G05.\}

$35 \mathrm{~S} 05$ General theory

Cardoso, Fernando

Petersen, Bent E.

$1 \mathrm{P}$

$35 \mathrm{~S} 10$ Initial value problems

$35 \mathrm{~S} 15$ Boundary value problems

$35 \$ 99$ None of the above, but in this section

\section{9-XX FINITE}

\section{DIFFERENCES AND} FUNCTIONAL EQUATIONS

39-00 Difficult to classify at the second level (must also be assigned at least one other classification number in this section)

39-01 Elementary exposition (collegiate level)

39-02 Advanced exposition (research surveys, etc.)

39-03 Historical

39-04 Explicit machine computation and programs (not the theory of computation or programming)

39A05 Finite differences, general

39A10 Difference equations

Fort, Tomlinson
39A15 Functional equations, general [For functional-differential equations, see 34Jxx.] $\begin{array}{ll}\text { Turdza, Erwin } & \text { 1P } \\ \text { Athreya, Krishna Balasundaram } & \text { IS }\end{array}$

39A20 Classical functional equations 39A25 Linear and multilinear functional equations

$$
\begin{array}{ll}
\text { Brydak, Dobieslaw } & \text { 1P } \\
\text { Salehi, Habib } & \text { 2P }
\end{array}
$$

39A30 Functional equations in several variables, systems

39A35 Matrix functional equations

39A40 Functional equations on abstract structures

40-XX SEQUENCES, SERIES, SUMMABILITY

$40-00$ Difficult to classify at the second level (must also be assigned at least one other classification number in this section)

40-01 Elementary exposition (collegiate level)

40-02 Advanced exposition (research surveys, etc.)

40-03 Historical

40-04 Explicit machine computation and programs (not the theory of computation or programming)

40Axx Convergence and divergence of infinite limiting processes

40A05 Convergence and divergence of series and sequences

$\begin{array}{ll}\text { Tonne, Philip C. } & \text { is } \\ \text { Franks, R. L. } & \text { is } \\ \text { Marzec, R. P. } & \text { is }\end{array}$

40A10 Convergence and divergence of integrals

40A15 Convergence and divergence of continued fractions [See also 30A22.]

$\begin{array}{ll}\text { Hag, Kari } & \text { is } \\ \text { Magnus, Arne } & 1 \mathrm{P} \\ \text { Roach, F. A. } & 2 \mathrm{P}\end{array}$

40A20 Convergence and divergence of infinite products

40A25 Approximation to limiting values (summation of series, etc.) [For the

Euler-Maclaurin summation formula, see 65B15.]

40A99 None of the above, but in this 
section

Menon, K. V.

2P

40B05 Multiple sequences and series

40Cxx General summability methods

40C05 Matrix methods

$\begin{array}{ll}\text { Bustoz, Joaquin } & 1 \mathrm{P} \\ \text { Atalla, Robert E. } & 1 \mathrm{P} \\ \text { Skerry, Herbert } & 1 \mathrm{P} \\ \text { Roach, F. A. } & 1 \mathrm{P} \\ \text { Fridy, J. A. } & 1 \mathrm{P} \\ \text { Atalla, Robert E. } & 3 \mathrm{P} \\ \text { Rainwater, John } & 2 \mathrm{P}\end{array}$

$40 \mathrm{C} 10$ Integral methods

$40 \mathrm{C} 15$ Function theoretic methods (including power series methods and semicontinuous methods)

Hartmann, Frederick

Lorch, Lee

$40 C 99$ Jone of the above, but in this section

40Dxx Direct theorems on summability

40D05 General theorems

Skerry, Herbert

40D10 Tauberian constants and oscillation limits

40D15 Convergence factors and summability factors

40D20 Summability and bounded fields of methods

\section{Atalla, Robert E.}

Rainwater, John

$3 \mathbf{S}$

40D25 Inclusion and equivalence theorems

$\begin{array}{ll}\text { Hartmann, Frederick } & \text { 1P } \\ \text { Shawyer, Bruce Lockhart Robertson } & \text { 1P } \\ \text { Yang, Gou-sheng } & \text { 1P } \\ \text { Shawyer, Bruce Lockhart Robertson } & \text { 2P }\end{array}$

40D99 None of the above, but in this section

Lorch, Lee

40E05 Tauberian theorems

Shea, Daniel F.

Saari, Donald G.

Pollard, Harry

Saari, Donald G.

Dikshit, H. P.

Leviatan, Dany
40F05 Absolute and strong summability

Borwein, David

Fridy, J. A.

Singh, Sheo Ram

40Gxx Special methods of summability

40G05 Cesàro, Euler, Nörlund and Hausdorff methods

Dikshit, H. P.

Singh, Sheo Ram

$3 S$

$1 \mathrm{P}$

40G10 Abel, Borel and power series methods

$\begin{array}{ll}\text { Shawyer, Bruce Lockhart Robertson } & \text { 1P } \\ \text { Borwein, David } & \text { 1P } \\ \text { Yang, Gou-sheng } & \text { 1P } \\ \text { Shawyer, Bruce Lockhart Robertson } & \text { 2P }\end{array}$

40G99 None of the above, but in this section

Hartmann, Frederick

Skerry, Herbert

1S

40H05 Functional analytic methods in summability

$\begin{array}{ll}\text { Bustoz, Joaquin } & \text { 1P } \\ \text { Atalla, Robert E. } & 1 P \\ \text { Kerr, Donald R., Jr. } & \text { 1S } \\ \text { Stratton, Howard H., Jr. } & \text { 1S } \\ \text { Brown, Herbert I. } & \text { 1S } \\ \text { Brown, Herbert I. } & 2 \text { P } \\ \text { Wilansky, Albert } & \text { IS }\end{array}$

40J05 Summability in abstract structures \{See also 43A55.

Powell, Robert E.

Cox, Raymond $\mathrm{H}$.

Wood, Bruce

Brown, Herbert I

41-XX APPROXIMATIONS AND EXPANSIONS For all approximation theory in the complex domain, see $30 \mathrm{~A} 80$ and 30A82; for all trigonometric approximation and interpolation, see 42A08, 42A12.

41-00 Difficult to classify at the second level (must also be assigned at least one other classification number in this section)

41-01 Elementary exposition (collegiate level) 
41-02 Advanced exposition (research surveys, etc.)

41-03 Historical

41-04 Explicit machine computation and programs (not the theory of computation or programming)

41A05 Interpolation [See also 42A12 and 65D05.]

41A10 Approximation by polynomials [For approximation by trigonometric polynomials, see $42 \mathrm{A08}$.]

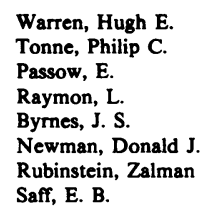

41A15 Spline approximation

41A20 Approximation by rational functions

$$
\text { Saff, E. B. } 2 P
$$

41A25 Rate of convergence, degree of approximation

$\begin{array}{ll}\text { Raymon, L. } & \text { 1P } \\ \text { Passow, E. } & 1 \mathrm{P} \\ \text { Prosser, Reese T. } & \text { IS }\end{array}$

41A30 Approximation by other special function classes

Peek, Darwin E.

41A35 Approximation by operators (in particular, by integral operators)

41A40 Saturation classes

41A45 Approximation by arbitrary linear and nonlinear expressions; widths and entropy

Jerome, Joseph W.
Schumaker, Larry L.

Prosser, Reese T.

1P

is

41A50 Best approximation (Čebyšev, etc.)

Kruskal, Joseph B.

Byrnes, J. S.

Newman, Donald J.

Hoffmann, Laurence D.

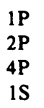

41A55 Approximate quadratures

41A60 Asymptotic approximations, asymptotic expansions (steepest descent, etc.) [See also 30A84.]

Nelson, Stuart

41A63 Multidimensional problems

Warren, Hugh E.

$1 \mathbf{P}$
41A65 Abstract approximation theory (approximation in normed linear spaces and other abstract spaces)

Singer, Ivan

Restrepo, Guillermo

$1 P$

42-XX FOURIER ANALYSIS

42-00 Difficult to classify at the second level (must also be assigned at least one other classification number in this section)

42-01 Elementary exposition (collegiate level)

42-02 Advanced exposition (research surveys, etc.)

42-03 Historical

42-04 Explicit machine computation and programs (not the theory of computation or programming)

42A04 Trigonometric polynomials, inequalities, extremal problems

$$
\begin{array}{ll}
\text { Beller, E. } & 1 P \\
\text { Beller, E. } & 2 P
\end{array}
$$

42A08 Approximation by trigonometric polynomials

42A12 Trigonometric interpolation

42A16 Fourier coefficients, Fourier series of functions with special properties, special Fourier series

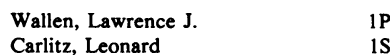

42A20 Convergence of Fourier and trigonometric series

Park, Chul

$1 \mathbf{P}$

42A24 Summability of trigonometric series

Dikshit, H. P.
Kwee, Bun-kung

$1 \mathrm{P}$
$1 \mathrm{P}$

42A28 Absolute convergence, absolute summability

Dikshit, H. P.

$2 \mathrm{P}$

42A32 Trigonometric series of special types (positive coefficients, monotonic coefficients, etc.)

42A36 Probabilistic methods in Fourier analysis

42A40 Conjugate functions, conjugate series, singular integrals 
Dikshit, H. P.

is

42A44 Lacunary series of trigonometric and other functions

42A48 Uniqueness of trigonometric expansions, uniqueness of Fourier expansions, Riemann theory, localization

$$
\text { Cooke, Roger }
$$

42A52 Orthogonal functions and polynomials, general theory [See also 33A 65.]

Al-Salam, Waleed A.
Muckenhoupt, Benjamin
Wilson, M. Wayne
Das, Minaketan
Wade, William R.

42A56 Fourier series in special orthogonal functions (Legendre polynomials, Walsh functions, etc.)

$$
\begin{aligned}
& \text { Zink, Robert E. } \\
& \text { McLaughlin, James R. } \\
& \text { Wells, Benjamin B., Jr. } \\
& \text { Wade, William R. }
\end{aligned}
$$

Cambanis, Stamatis

$2 \mathrm{P}$
$1 \mathrm{P}$
$1 \mathrm{P}$
$1 \mathrm{P}$
$1 \mathrm{P}$

42A60 Series of general orthogonal functions and generalized Fourier expansions

$$
\begin{aligned}
& \text { Newman, Donald J. } \\
& \text { Byrnes, J. S. } \\
& \text { Seeley, Robert T. } \\
& \text { Park, Chul } \\
& \text { Wilson, M. Wayne }
\end{aligned}
$$

42A62 Uniqueness and localization for orthogonal series

42A64 Completeness of sets of functions

Cambanis, Stamatis

Ginsberg, Jonathan I.

Chang, John S. M.

42A68 Fourier transforms

Wallen, Lawrence J.

Busenberg, Stavros

1P

42A72 Fourier-Stieltjes transforms [See also 60E05.]

Friedberg, Stephen Nelson, Stuart

42A76 Other transforms of Fourier type [See also 44A60.]

42A80 Trigonometric moment problems

42A84 Classical almost periodic functions [See also 43A60.]

Sibuya, Yasutaka

42A88 Positive definite functions

Einhorn, Sheldon J.

42A92 Multiple Fourier series and integrals

$\begin{array}{ll}\text { Schwartz, Alan L. } & \text { 2P } \\ \text { Nelson, Stuart } & 2 P \\ \text { Cooke, Roger } & 1 P\end{array}$

42A96 Convolution, factorization

\section{3-XX ABSTRACT}

HARMONIC ANALYSIS \{For other analysis on topological and Lie groups, see 22Exx. \}

43-00 Difficult to classify at the second level (must also be assigned at least one other classification number in this section)

\section{3-01 Elementary exposition} (collegiate level)

43-02 Advanced exposition (research surveys, etc.)

43-03 Historical

$43 \mathrm{~A} 05$ Measures on groups and semigroups

Tam, K. W.
Jamison, Benton
Orey, Steven
Atalla, Robert E.
Doss, Raouf
Tserpes, Nicholas
Mukherjea, Arunava
Joffe, A. D.

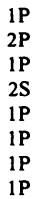

43A07 Means on groups and semigroups

$\begin{array}{ll}\text { Chou, Ching } & \text { 1P } \\ \text { Chou, Ching } & \text { 2P } \\ \text { Tserpes, Nicholas } & \text { IS } \\ \text { Mukherjea, Arunava } & \text { 1S } \\ \text { Chou, Ching } & \text { 3P }\end{array}$
43A10 Measure algebras on groups and semigroups

Pym, John S.

Baker, John Warren

Graham, Colin C.

$43 \mathrm{~A} 15 \mathrm{~L}_{\mathrm{p}}$ spaces and other function spaces on groups and semigroups

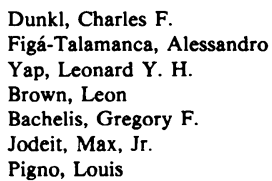

$43 \mathrm{~A} 20 \mathrm{~L}_{1}$ algebras on groups and semigroups

Butt, Shu-shih

Dunkl, Charles F.

Wood, Geoffrey V.

Baker, John Warren

Bachelis, Gregory F.

\section{P 政}

\section{a}


Rider, D. G.

$1 \mathrm{P}$
$1 \mathrm{P}$
$1 \mathrm{~S}$

Saeki, Sadahiro

43A22 Homomorphisms and multipliers of function spaces on groups

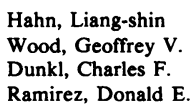

43A25 Fourier and Fourier-Stieltjes transforms on locally compact abelian groups

Chaney, Robin W.
Butt, Shu-shih
Figá-Talamanca, Alessandro
Graham, Colin C.
Wang, Ju-kwei
Friedberg, Stephen
Friedberg, Stephen
Saeki, Sadahiro

43A30 Fourier and Fourier-Stieltjes transforms on nonabelian groups and on semigroups

Figá-Talamanca, Alessandro
Doss, Raouf

$1 \mathbf{P}$
$1 \mathbf{P}$
$1 \mathbf{P}$

Rider, D. G.

43A32 Other transforms and operators of Fourier type

43A35 Positive definite functions on groups

43A40 Character groups and dual objects

Armacost, David Lee

Joffe, A. D.

2P

43A45 Spectral synthesis on groups and semigroups

Schwartz, Alan L.

Saeki, Sadahiro

$2 \mathrm{P}$
$1 \mathrm{P}$

43A50 Convergence of Fourier series and of inverse transforms

Bachelis, Gregory F.

Onneweer, C. W.

43A55 Summability methods on groups and semigroups

43A60 Almost periodic functions on groups and semigroups

43A65 Representations of groups and semigroups

43A70 Analysis on specific locally compact abelian groups [See also 12A85.]

Graham, Colin C.

$2 \mathrm{P}$

43A75 Analysis on specific compact groups

Dunkl, Charles F

$1 \mathbf{P}$
Onneweer, C. W.

Joffe, A. D.

1P

43A80 Analysis on other specific Lie groups

43A85 Analysis on homogeneous spaces

43A90 Spherical functions

43A95 Categorical methods [See also $18-\mathrm{XX}$.]

44-XX INTEGRAL

TRANSFORMS, OPERATIONAL CALCULUS \{For fractional derivatives and integrals, see 26A33.

44-00 Difficult to classify at the second level (must also be assigned at least one other classification number in this section)

44-01 Elementary exposition (collegiate level)

44-02 Advanced exposition (research surveys, etc.)

44-03 Historical

44-04 Explicit machine computation and programs (not the theory of computation or programming)

44A05 General transforms [See also 42A76.]

44A10 Laplace transform

44A15 Special transforms (Legendre, Hilbert, etc.)

$$
\begin{aligned}
& \text { Shea, Daniel F. } \\
& \text { Schwartz, Alan L. } \\
& \text { Fox, Charles }
\end{aligned}
$$

44A20 Transforms of special functions

44A25 Singular integrals (Calderó

n-Zygmund, etc.) [See also 47G05.]

Walsh, $T$.

$1 \mathrm{P}$

44A30 Multiple transforms

44A35 Convolution

44A40 Calculus of Mikusiński and other similar operational calculi

44A45 Classical operational calculus

Kulshreshtha, S. K.

Arora, Kasturi L.

$1 \mathrm{P}$
$1 \mathrm{P}$

44A50 Moment problems [See also 42A80.]

\section{5-XX INTEGRAL}




\section{EQUATIONS}

45-00 Difficult to classify at the second level (must also be assigned at least one other classification number in this section)

45-01 Elementary exposition (collegiate level)

45-02 Advanced exposition (research surveys, etc.)

45-03 Historical

45-04 Explicit machine computation and programs (not the theory of computation or programming)

45A05 Linear integral equations

Helton, Burrell, w. Lovelady, David Lowell $2 \mathbf{P}$
$2 \mathbf{P}$

45B05 Fredholm integral equations Kalaba, Robert E. $1 P$

45C05 Eigenvalue problems \{See also 34B25, 35Pxx, 47E05.

45D05 Volterra integral equations \{See also 34A10.

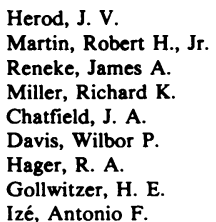

$1 P$
$1 P$
$1 P$
$1 P$
$1 S$
$1 S$
$1 P$
$3 P$
$1 P$

45Exx Singular integral equations 45E05 Integral equations with Cauchy type kernels [See also 30A88, 35J15, 44A15, 44A35.]

Miranda, Guillermo

$45 \mathrm{E} 10$ Integral equations of the convolution type (Abel, Picard, Toeplitz and Wiener-Hopf type) [See also 44A15, 44A35, 47B35.]

\section{Kraut, Edgar A.}

Busenberg, Stavros

45E99 None of the above, but in this section

Miranda, Guillermo

$1 \mathrm{P}$

45F05 Systems of linear integral equations
45Gxx Nonlinear integral equations (See also $47 \mathrm{Hxx}$. 45G05 Singular nonlinear integral equations

45G99 Other nonlinear integral equations

Hager, R. A.

Gollwitzer, H. E.

Izé, Antonio F.

Hess, Peter

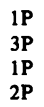

45H05 Miscellaneous special kernels \{See also 44A15.

Fox, Charles

$1 \mathrm{P}$

\section{J05 Integro-ordinary differential equations}

\section{K05 Integro-partial differential equations}

45Lxx Approximation of solutions 45L05 Theoretical approximation of solutions

45L10 Numerical approximation of solutions [For numerical analysis, see 65R05.]

45Mxx Qualitative behavior

\section{M05 Asymptotics}

Izé, Antonio F.

45M10 Stability theory

Lovelady, David Lowell

45M99 None of the above, but in this section

45N05 Abstract integral equations, integral equations in abstract spaces

46-XX FUNCTIONAL

ANALYSIS \{For manifolds modelled on topological linear spaces, see 57A20, 58Bxx. \} 46-00 Difficult to classify at the second level (must also be assigned at least one other classification number in this section)

46-01 Elementary exposition (collegiate level)

46-02 Advanced exposition (research surveys, etc.) 
46-03 Historical

46-04 Explicit machine computation and programs (not the theory of computation or programming)

\section{Axx Topological linear spaces}

46A05 Locally convex spaces

Curtis, Douglas W.
Washenberger, James K.
Vidossich, Giovanni
Johnson, Dudley Paul
Brown, Herbert I.
Jain, N. C.
Cochran, Allan C.
Saxon, Stephen
Walsh, B. J.
Chou, Ching
Hosack, John M.

46A07 Barrelled spaces

Saxon, Stephen Levin, Mark

46A09 Bornological spaces

Cochran, Allan C.

46A10 Locally bounded topological linear spaces

46A15 Other topological linear spaces

Kascic, Michael J., Jr.

Shapiro, J. H.

Gregory, D. A.

Saxon, Stephen

46A20 Duality theory

Dollinger, Michael B.

$1 \mathrm{P}$

46A25 Reflexivity and semi-reflexivity

46A30 Open mappings and closed graph theorems and completeness (including B-, $\mathrm{B}_{\mathrm{r}}$-completeness)

Harvey, Charles
Harvey, Reese
Saxon, Stephen

46A35 Summability and bases

$$
\begin{aligned}
& \text { Dyer, James A. } \\
& \text { Johnson, W. B. } \\
& \text { Cook, Thurlow A. } \\
& \text { Gellar, Ralph } \\
& \text { Kalton, N. J. } \\
& \text { Hofler, John T. }
\end{aligned}
$$

1P
is
is
is
$2 \mathrm{P}$
1S
1S
$2 \mathrm{P}$
1S
$3 \mathrm{P}$
$2 \mathrm{P}$

46A40 Ordered topological linear spaces, vector lattices

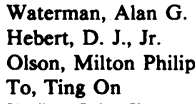

46A45 Sequence spaces (including Kö the spaces)

$\begin{array}{ll}\text { Atalla, Robert E. } & \text { 1P } \\ \text { Bustoz, Joaquin } & \text { 1P } \\ \text { Gellar, Ralph } & 1 \mathrm{~S} \\ \text { Tong, Alfred E. } & 1 \mathrm{P} \\ \text { Brown, Herbert I. } & 2 \mathrm{P}\end{array}$

46A99 None of the above, but in this section

46Bxx Normed linear spaces and Banach spaces (For function spaces, see 46Exx.

46B05 Topology in terms of the norms

McCoy, R. A.

Jain, N. C.

Stein, James D., Jr.

46B10 Duality and reflexivity

Uhl, J. J., Jr.

Holub, J. R.

1S

46B15 Summability and bases

$\begin{array}{ll}\text { Zink, Robert E. } & \text { 1P } \\ \text { Davis, William J. } & \text { 1P } \\ \text { Holub, J. R. } & \text { 1P } \\ \text { Holub, J. R. } & \text { 2P } \\ \text { Jain, N. C. } & \text { 1P } \\ \text { Merryman, Emory Hughes } & \text { 1P } \\ \text { Zippin, M. } & \text { 2P } \\ \text { Johnson, W. B. } & \text { 2P } \\ \text { Warren, Hugh E. } & \text { 2P }\end{array}$

46B99 None of the above, but in this section

$\begin{array}{ll}\text { Pothoven, Kenneth } & \text { 1P } \\ \text { Jerome, Joseph W. } & \text { IP } \\ \text { Schumaker, Larry L. } & \text { IP } \\ \text { Johnson, Dudley Paul } & \text { 1S } \\ \text { Bilyeu, Russell, G. } & \text { 1P } \\ \text { Torrance, Ellen } & \text { 2P } \\ \text { Tzafriri, L. } & \text { 2P } \\ \text { Howland, James S. } & \text { 2P } \\ \text { Holub, J. R. } & \text { 3S } \\ \text { Koehler, Donald O. } & \text { IP }\end{array}$

46Cxx Inner product spaces, Hilbert spaces (For function spaces, see 46Exx.

$46 \mathrm{C05}$ Geometry and topology of the spaces

$\begin{array}{ll}\text { Kruskal, Joseph B. } & \text { 1P } \\ \text { Johnson, Gordon G. } & \text { 1P } \\ \text { Đokovič, Dragomir Ž. } & \text { 2S } \\ \text { Holbrook, John A. R. } & \text { 1S } \\ \text { Wong, Pak-ken } & \text { 3P }\end{array}$

$46 \mathrm{C} 10$ Other properties of such spaces

$\begin{array}{ll}\text { MacNerney, J. S. } & \text { IP } \\ \text { Kuelbs, J. D. } & \text { IP } \\ \text { Moore, Berrien, III } & \text { is } \\ \text { Giellis, George R. } & \text { IS }\end{array}$

46D05 Spaces with indefinite inner 
product

46Exx Function spaces \{For function algebras, see $46 \mathrm{~J} 10$.

$46 \mathrm{E} 05$ Lattices of continuous, differentiable or analytic functions

Morris, Peter D.
Lacey, H. Elton
Shore, Samuel D.

46E 10 Topological linear spaces of continuous, differentiable or analytic functions

$\begin{array}{ll}\text { Atalla, Robert E. } & 1 S \\ \text { Bustoz, Joaquin } & 1 S \\ \text { Jain, N. C. } & 2 S \\ \text { Wayment, S. G. } & 3 P \\ \text { Edwards, J. R. } & 2 P \\ \text { Edwards, J. R. } & 3 P\end{array}$

46E15 Banach spaces of continuous, differentiable or analytic functions

Katz, Melvin L, Jr.
Friedman, Neal
Cima, Joseph A.
Kellogg, C. N.
Jamison, Benton
Ginsberg, Jonathan I.
Asimow, Leonard
Atalla, Robert E.
Hoffmann, Laurence D.
Warren, Hugh E.
Wilansky, Albert
Lacey, H. Elton

46E20 Hilbert spaces of continuous, differentiable or analytic functions

Schubert, C. F.

Cambanis, Stamatis

46E25 Rings and algebras of continuous, differentiable or analytic functions [For Banach function algebras, see $46 \mathrm{~J} 10$, 46J15.]

Wilken, Donald R.

46E30 Spaces of measurable functions, $L^{p}$ spaces, Orlicz spaces [See also 43A 15.]

Newman, Donald J.
Byrnes, J. S.
Zink, Robert E.
Sundaresan, Kondagunta
Zippin, M.
Etter, Daniel O., Jr.
Cherkas, Barry M.
Tzafriri, L.
Halpern, Benjamin R.
Schneeberger, C.
Lacey, H. Elton

46E35 Sobolev spaces, embedding theorems, trace theorems, interpolation spaces

$\begin{array}{ll}\text { Strichartz, Robert S. } & \text { 1P } \\ \text { Adams, Robert A. } & \text { IP } \\ \text { Harvey, Reese } & \text { IS } \\ \text { Harvey, Charles } & \text { IS } \\ \text { Strichartz, Robert S. } & \text { 2P }\end{array}$

$46 \mathrm{E} 40$ Spaces of vector- and operator-valued functions

$\begin{array}{ll}\text { Sundaresan, Kondagunta } & \text { IP } \\ \text { Leech, Robert B. } & \text { IP } \\ \text { Goodrich, Robert K. } & \text { IP }\end{array}$

46E99 None of the above, but in this section

46Fxx Distributions, generalized functions, distribution spaces

46F05 Topological linear spaces of test functions and distributions

$$
\text { Dudley, R. M. } 1 \text { P }
$$

46F10 Operations with distributions

$$
\text { Weinstock, Barnet } M \text {. } 2 S
$$

46F15 Hyperfunctions, analytic functionals [See also 32A25, 32C35.]

Howland, James S

46F99 None of the above, but in this section

Kritt, B.

$1 \mathrm{~S}$

46Gxx Measures, integration, derivatives (For integration on infinite-dimensional spaces, see 28A40; for nonlinear functional analysis, see $58-\mathrm{XX}$, especially

$58 \mathrm{Cxx}$.

46G05 Derivatives

Uhl, J. J., Jr.
Goodman, Victor

46G10 Vector-valued measures and integration

$$
\begin{aligned}
& \text { Uhl, J. J., Jr. } \\
& \text { Johnson, Gerald W. } \\
& \text { Goodrich, Robert K. } \\
& \text { Johnson, Dudley Paul } \\
& \text { Uhl, J. J., Jr. } \\
& \text { Walsh, B. J. }
\end{aligned}
$$$$
\begin{aligned}
& \text { IP } \\
& \text { 1P } \\
& \text { 1P } \\
& \text { 1S } \\
& \text { 3P } \\
& \text { 1P }
\end{aligned}
$$

$46 \mathrm{G} 15$ Lifting theory

46G99 None of the above, but in this section

46Hxx Topological algebras, normed rings and algebras, Banach algebras \{For group algebras, 
convolution algebras and measure algebras, see $43-X X$.

46H05 General theory

Grabiner, Sandy
Collins, Heron Sherwood
Summers, W. H.
Chernoff, Paul R.
Gelbaum, Bernard R.
Etter, Daniel O., Jr.
Sinclair, Allan M.
Carpenter, R. L.
Cochran, Allan C.

46H10 Ideals and subalgebras

Gelbaum, Bernard R.

Laursen, Kjeld B.

Torrance, Ellen

Elliott, George A.

46H15 Representations

Johnson, Gerald W.

Gellar, Ralph

46H20 Structure, classification

Cochran, Allan C.

Cochran, Allan C.

$46 \mathrm{H} 25$ Normed modules and Banach modules

Summers, W. H.
Collins, Heron Sherwood
Ramirez, Donald E.
Dunkl, Charles F.
Giellis, George R.

$46 \mathrm{H} 99$ None of the above, but in this section

Spatz, I. N.
Wong, Pak-ken
Sibuya, Yasutaka
Sinclair, Allan M.

Sinclair, Allan M
Carpenter, R. L.

46Jxx Commutative Banach algebras

46J05 General theory

Allan, G. R.

Lindberg, John A., Jr.

IP

$46 \mathrm{~J} 10$ Banach algebras of continuous functions, function algebras

König, Heinz

Dietrich, William E., Jr.

Restrepo, Guillermo

Eifler, Larry

Gonshor, Harry

Blumenthal, Robert George

Natzitz, Boaz

Itô, Takashi

Sidney, S. J.

Sidney, S. J.

Warren, Hugh E.

Luchins, Edith $\mathrm{H}$.
Brandstein, A. G.

Strichartz, Robert S.

$1 \mathrm{P}$

1P

46J15 Banach algebras of differentiable or analytic functions, $\mathrm{H}^{\mathrm{P}}$-spaces [See also 30A98, 32E25.]
$1 P$
$1 P$
$1 P$

Davie, Alexander $M$

46J20 Ideals, maximal ideals, boundaries

Dietrich, William E., Jr.

Asimow, Leonard

Blumenthal, Robert George

Brandstein, A. G.

IP

$1 \mathrm{P}$

IP

46J25 Representations

46J30 Subalgebras

Brown, Leon

$1 \mathbf{P}$

46J35 Structure, classification

$46 \mathrm{~J} 99$ None of the above, but in this section

Ting, Wei-lung

Luchins, Edith $\mathrm{H}$

Rider, D. G.

46Kxx Rings and algebras with an involution

46K05 Ideals, subalgebras

46K10 Representations

46K 15 Hilbert algebras

Friedell, J. C

Saworotnow, Parfeny P.

Saworotnow, Parfeny P.

Giellis, George R.

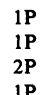

46K99 None of the above, but in this section

Sinclair, Allan M.
Wong, Pak-ken

$3 \mathrm{~S}$

46Lxx Rings and algebras of operators, with or without involution \{For other algebraic systems of operators, see 47Dxx.

46L05 $C^{*}$-algebras ( = $\mathrm{B}^{*}$-algebras) [See also 22D25.]

Aarnes, Johan F.

Kadison, Richard V.

Ono, Tamio

Bunce, John

Sinclair, Allan $\mathbf{M}$

Wong, Pak-ken

Torrance, Ellen

Andersen, Tage Bai

Reid, James L.

Elliott, George A

Bunce, John

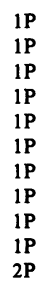


Barnes, Bruce Alan

$1 \mathrm{P}$

46L10 von Neumann algebras ( = rings of operators, $\mathrm{W}^{*}$-algebras)

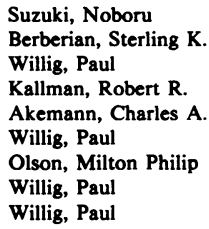

$3 \mathrm{P}$
$1 \mathrm{P}$
$1 \mathrm{P}$
$2 \mathrm{P}$
$1 \mathrm{P}$
$2 \mathrm{P}$
$1 \mathrm{P}$
$3 \mathrm{P}$
$4 \mathrm{P}$

46L15 Nonselfadjoint operator algebras on Hilbert space

Lambert, Alan

$1 \mathrm{P}$

46L20 Operator algebras on Banach and linear topological spaces

Brown, Herbert I.

Stratton, Howard H., Jr.

Kerr, Donald R., Jr.

Wilansky, Albert

Barnes, Bruce Alan

$1 \mathrm{P}$
$1 \mathrm{P}$
$1 \mathrm{P}$
$1 \mathrm{P}$
$1 \mathrm{P}$

46L25 Dual spaces of operator algebras and topological groups

Busby, Robert C.

$1 \mathrm{P}$

46L99 None of the above, but in this section

Gilfeather, Frank

Helton, Burrell, w.

$1 \mathrm{P}$

Catlin, Donald E.

1S

46Mxx Categorical methods (See also $18-\mathrm{XX}$.

46M05 Tensor products

Baker, J. M.

Gelbaum, Bernard R.

Howland, James S.

1S

2S

46M10 Projective and injective objects

Pothoven, Kenneth

Gonshor, Harry

1P

46M15 Functors

46M99 None of the above, but in this section

Ting, Wei-lung

$1 \mathrm{P}$

46N05 Miscellaneous applications of functional analysis

Chou, Ching

$3 \mathrm{~S}$

47-XX OPERATOR THEORY

47-00 Difficult to classify at the second level (must also be assigned at least one other classification number in this section)
47-01 Elementary exposition

(collegiate level)

47-02 Advanced exposition (research surveys, etc.)

$47-03$ Historical

47-04 Explicit machine computation and programs (not the theory of computation or programs)

47Axx Single linear operators: general theory

47A05 Adjoints, conjugates

Wilansky, Albert

Holbrook, John A. R.

1S

47A10 Spectrum, resolvent, numerical range

Berberian, Sterling $\mathrm{K}$

Hosack, John M.

Gilfeather, Frank

Fink, James P.

Gustafson, Karl

Bunce, John

Chow, T. R.

Prosser, Reese T.

Sinclair, Allan $M$.

Putnam, Calvin R.

Bouldin, Richard

Furuta, Takayuki

Nakamoto, Ritsuo

Bunce, John

Holbrook, John A. R.

Koehler, Donald O.

47A15 Invariant subspaces

Radjavi, Heydar

Rosenthal, Peter

Radjavi, Heydar

Caradus, S. R.

Nordgren, Eric A.

Williams, James $P$.

Harrison, K. J.

Rosenthal, Peter

Radjavi, Heydar

Deddens, James A

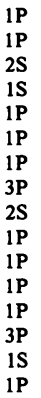

47A20 Dilations, extensions, compressions

Crandall, Michael G.

Gilbert, Richard C.

Hosack, John M.

Chow, T. R.

Gilfeather, Frank

Jones, Lee

Kuftinec, Velimir

47A25 Spectral sets

Clancey, Kevin F.

1P

47A30 Norms (inequalities, more than one norm, etc.)

Nakamoto, Ritsuo

Furuta, Takayuki

$1 P$
$1 P$ 
47A35 Ergodic theory [See also 28A65.]

Baxter, J. R.

Jones, Lee

Kuftinec, Velimir

Kuftinec, Velimir

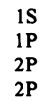

47A40 Scattering theory [See also 35J10.]

47A45 Canonical models for contractions and nonselfadjoint operators

Gilbert, Richard C.

Leech, Robert B.

Caradus, S. R.

Deddens, James A.

47A50 Equations involving linear operators

Gilfeather, Frank

Dotson, W. G., Jr.

Riddell, R. C.

Deddens, James A.

Salehi, Habib

Hess, Peter

47A55 Perturbation theory

Lavine, Richard B.

Warner, Kenneth $\mathbf{K}$

Greenlee, W. M

Kazdan, Jerry L.

Prosser, Reese T.

Howland, James S.

47A60 Functional calculus

Finkelstein, $\mathbf{M}$.

Chow, T. R.

Kantorovitz, Shmue

Kazdan, Jerry L.

Kritt, B.

47A65 Structure theory

$\begin{array}{ll}\text { Constantin, Gheorghe } & 1 \mathrm{P} \\ \text { Istrăţescu, Ioana } & 1 \mathrm{P} \\ \text { Foguel, Shaul Reuven } & 1 \mathrm{P} \\ \text { Pincus, Joel David } & 1 \mathrm{P} \\ \text { Ornstein, Donald S. } & 2 \mathrm{P} \\ \text { Gilfeather, Frank } & 2 \mathrm{~S} \\ \text { Kritt, B. } & 1 \mathrm{P}\end{array}$

47A70 Eigenfunction expansions, rigged Hilbert spaces

Kuelbs, J. D.

47A99 None of the above, but in this section

47Bxx Single linear operators: special classes of operators

47B05 Compact operators, Riesz operators

Constantin, Gheorghe

Istrățescu, Ioana

Summers, W. H.

Collins, Heron Sherwood

Gilfeather, Frank
Tong, Alfred E.

Gilfeather, Frank

Rhoads, Donald

Prosser, Reese T.

Lin, C.-S.

Wilansky, Albert

Patel, S. M.

Ramanujan, P. B.

1S
$2 P$
$1 P$
$3 P$
$1 P$
$1 P$
$1 S$
$1 S$

47B10 Hilbert-Schmidt operators, trace class operators, nuclear operators, etc.

$\begin{array}{ll}\text { Strait, Peggy Tang } & \text { 2S } \\ \text { Friedell, J. C. } & \text { 1P } \\ \text { Saworotnow, Parfeny P. } & \text { 1P } \\ \text { Saworotnow, Parfeny P. } & \text { 2P } \\ \text { Clancey, Kevin F. } & \text { 2P } \\ \text { Kuelbs, J. D. } & 1 \text { P } \\ \text { Holub, J. R. } & \text { 3P }\end{array}$

47B15 Hermitian and normal operators (spectral measures, functional calculus, etc.)

$\begin{array}{ll}\text { Berberian, Sterling K. } & \text { 2P } \\ \text { Kazdan, Jerry L. } & \text { 1P } \\ \text { Kritt, B. } & \text { 1P }\end{array}$

47B20 Subnormal operators, hyponormal operators, etc.

Constantin, Gheorghe

Istrăţescu, Ioana

Suzuki, Noboru

Warner, Kenneth K.

Clancey, Kevin F.

Bunce, John

Putnam, Calvin R.

Lambert, Alan

Bunce, John

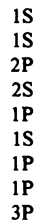

47B25 Symmetric and selfadjoint operators (unbounded)

Gilbert, Richard C.

Poulsen, Ebbe Thue

Fink, James $P$.

Kazdan, Jerry L.

$2 \mathrm{~S}$
$1 \mathrm{P}$
$2 \mathrm{P}$
$2 \mathrm{P}$
$1 \mathrm{P}$
$3 \mathrm{P}$

7 B30 Fredholm operators

Washenberger, James $\mathrm{K}$.

Pfaffenberger, William

Douglas, Ronald George

Sarason, Donald

Bouldin, Richard

Hosack, John M.

1S
1S
$2 \mathrm{P}$
$2 \mathrm{~S}$
$1 \mathrm{P}$
$1 \mathrm{~S}$
$1 \mathrm{P}$
$1 \mathrm{P}$
$3 \mathrm{P}$

355 Toeplitz operators, Wiener-Hop operators [For other integral operators, see also $47 \mathrm{G} 05$.]

Surason, Donald

Douglas, Ronald George

Moore, Berrien, III

47B40 Spectral operators

Plafker, Stephen $\mathbf{M}$.

Kritt, B.

47B44 Dissipative operators

Packel, Edward W. 
Crandall, Michael G.

47B45 Difference operators

Williams, James $P$.

47B47 Commutators

Lavine, Richard B.

Pincus, Joel David

Lin, C.-S.

Schneeberger, C.

Patel, S. M

Ramanujan, P. B.
$1 \mathrm{~S}$

1P

47B50 Operators on a space with an indefinite metric

47B55 Operators on ordered spaces Huff, Robert E.

$1 \mathrm{P}$

47B99 None of the above, but in this section

Gellar, Ralph

Wilken, Donald $\mathbf{R}$.

Nordgren, Eric A.

W:lliams, James P.

At.ila, Robert E.

Ramanujan, P. B.

Patel, S. M.

Walsh, B. J.

Koehler, Donald O.

47Cxx Single linear operators as elements of algebraic systems

47C05 Operators in algebras

Rosenthal, Peter

Harrison, K. J.

Radjavi, Heydar

Lambert, Alan

47C10 Operators in *-algebras

Andersen, Tage Bai

$47 \mathrm{C} 15$ Operators in von Neumann algebras

Gilfeather, Frank

Chow, T. R.

$3 P$
$2 P$

47699 None of the above, but in this section

47Dxx Algebraic systems of linear operators \{For rings and algebras of operators, see $46 \mathrm{Kxx}, 46 \mathrm{Lxx}$.

47D05 Semigroups of operators [For Markov processes, see $60 \mathrm{~J} \times \mathrm{x}$.]

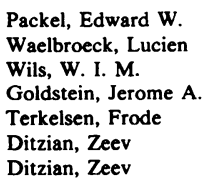

Packel, Edward W.

Waelbroeck, Lucien

Wils, W. I. M.

Goldstein, Jerome A

Terkelsen, Frode

Ditzian, Zeev

Ditzian, Zeev

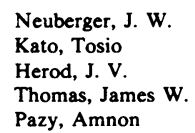

Kantorovitz, Shmuel

Kauffman, Robert $M$

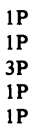

$1 P$

1P

$1 P$

$2 S$

47D15 Linear spaces of operators

Baker, J. M. IP

47D20 Convex sets and cones of operators

Sine, R. C.

2P

47D99 None of the above, but in this section

$\begin{array}{ll}\text { Uhl, J. J., Jr. } & \text { is } \\ \text { Pfaffenberger, William } & \text { 1P } \\ \text { Catlin, Donald E. } & \text { iS }\end{array}$

47E05 Ordinary differential operators \{See also 34B25, 58Fxx.

$\begin{array}{ll}\text { Zettl, Anton } & 2 S \\ \text { Kazdan, Jerry L. } & \text { 1S } \\ \text { Kauffman, Robert M. } & 2 P\end{array}$

47F05 Partial differential operators \{See also 35Pxx, 58Gxx.\}

47G05 Integral, integro-differential, and pseudodifferential operators \{ See also $35 \mathrm{Sxx}, 58 \mathrm{Gxx}$.
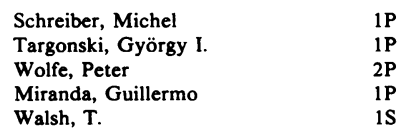

\section{Hxx Nonlinear operators}

47H05 Monotone operators

Hess, Peter

47H10 Fixed point theorems

Van Vleck, F. S.

Himmelberg, Charles J.

Pellicciaro, E. J.

Kirk, W. A.

Guseman, Lawrence F, Jr.

Kirk, W. A

Thomas, James W.

Franks, R. L.

Marzec, R. P.

47H15 Equations involving nonlinear operators

Herod, J. V.

Hess, Peter

3P

47 H99 None of the above, but in this 
section

Miyadera, Isao

Hess, Peter

49-XX CALCULUS OF

VARIATIONS AND OPTIMAL CONTROL \{ See also 34H05, 93Exx. ?

49-00 Difficult to classify at the second level (must also be assigned at least one other classification number in this section)

49-01 Elementary exposition (collegiate level)

49-02 Advanced exposition (research surveys, etc.)

49-03 Historical

49-04 Explicit machine computation and programs (not the theory of computation or programming)

49Axx Existence theory for optimal solutions

49A05 Free problems in one independent variable

49A10 Problems involving ordinary differential equations

49A15 Free problems in two or more independent variables

49A20 Problems involving partial differential equations

49A25 Problems in abstract spaces

49A30 Problems involving functional relations other than differential equations

49A35 Optimal solutions belonging to restricted classes (bang-bang controls, etc.)

49A40 Minimax problems

49A50 Topology of solutions, weak and strong minima, semicontinuity, convexity, orientor fields

49A99 None of the above, but in this section

\section{Bxx Necessary conditions and} sufficient conditions for optimality

49B05 Free problems in one independent variable

49B10 Problems involving ordinary differential equations
49B15 Optimal solutions belonging to restricted classes

49B20 Free problems in two or more independent variables

49B25 Problems involving partial differential equations

49B30 Problems in abstract spaces

49B35 Problems involving functional relations other than differential equations

49B40 Minimax problems

49B50 Sensitivity of optimal solutions in the presence of perturbations

49B99 None of the above, but in this section

\section{Cxx Caratheodory}

Hamilton-Jacobi theories, including

dynamic programming

49C05 Free problems and problems involving ordinary differential equations

49C10 Free problems and problems involving partial differential equations

49C15 Problems in abstract spaces or involving functional relations other than differential equations

49C99 None of the above, but in this section

\section{Dxx Methods of successive} approximations

49D05 Methods based on necessary conditions

49D10 Methods of steepest descent type 49D15 Methods of Newton-Raphson, Galerkin and Ritz types

49D20 Methods of relaxation type

49D25 Finite difference methods

49D30 Other methods, not based on necessary conditions (penalty function, etc.)

49D35 Methods of linear programming type

49D40 Methods of quadratic programming type

49D45 Methods of convex programming type

49D99 None of the above, but in this section 


\section{Exx Controllability and} geometry of control problems

49E05 General dependence on controls

49E10 Orientor fields (contingency equations)

49E15 Attainable sets, controllability

49E20 Interrelations between stability problems and optimization problems

49E25 Effect of perturbations on controllability

49E30 Relations between controllability and optimal solutions

49E99 None of the above, but in this section

\section{Fxx Manifolds}

49F05 Exterior differential forms, invariant integrals (Cartan theory) [See also 58A15.]

49F10 Minimal surfaces

49F15 Morse theory in Hilbert and other spaces [See also 58Exx.]

49F20 Geometric measure and integration theory, integral and normal currents, flat chains and cochains, varifolds [See also 58A25.]

49F22 Existence and structure of solutions to variational problems in geometric measure-theoretic setting

49F25 Surface area

49F99 None of the above, but in this section

49Gxx Variational methods and eigenvalues

49G05 Variational approach to eigenvalues

$$
\text { Hess, Peter }
$$

49G10 Rayleigh-Ritz methods

49G15 Weinstein and Aronszajn methods, intermediate problems

49G20 Linear operators in Hilbert spaces

49G99 None of the above, but in this section

49H05 Variational principles of physics \{See also 70Hxx, 81A09.\} 50-XX GEOMETRY \{For algebraic geometry, see $14-\mathrm{XX}$.

50-00 Difficult to classify at the second level (must also be assigned at least one other classification number in this section)

50-01 Elementary exposition (collegiate level)

50-02 Advanced exposition (research surveys, etc.)

50-03 Historical

50-04 Explicit machine computation and programs (not the theory of computation or programming)

\section{Axx Foundations}

50A05 Euclidean

Schwabhäuser, Wolfram

Gutiérrez-Novoa, Lino

50A10 Noneuclidean

50A15 Transformation groups

50A20 Algebraic characterizations [See also 06A75, 12K05, 16A76, 16A78, 16A80, 17D05, 17E05, 20N10.]

Schwabhäuser, Wolfram $1 P$

50A25 Models [See also 02H15.]

50A30 Length, area, volume

50A99 None of the above, but in this section

50Bxx Euclidean geometry (including equiform geometry)

50B05 Constructions

50B10 Metric formulae

50B 15 Inequalities

50B20 Geometry of circles

50B25 Euclidean and equiform geometry over fields other than the reals

50B30 Regular figures, division of space [See also 20H15.]

50B35 Other groups generated by reflections

50B99 None of the above, but in this section

$50 \mathrm{Cxx}$ Other metric geometries

$50 \mathrm{C05}$ Elliptic and hyperbolic, general

Valentine, Joseph E.

$1 \mathrm{P}$ 
$50 \mathrm{C10}$ Elliptic and hyperbolic inequalities

$50 \mathrm{C} 15$ Groups generated by elliptic and hyperbolic reflections [See also $20 \mathrm{H} 10$.]

50C20 Hyperbolic convexity

Fillmore, Jay $\mathbf{P}$

$1 \mathrm{P}$

$50 \mathrm{C} 25$ Other metric geometries Petty, C. M.

50Dxx Geometries of other transformation groups

50D05 Affine geometry, general

Yeh, R. Z.

Johnson, Norman Lloyd

50D10 Affine geometry, subgroups (centroaffine, equiaffine)

50D15 Descriptive geometry

50D20 Projective geometry over the reals

50D25 Projective geometry over other infinite fields

50D30 Projective geometry over finite fields

50D35 Projective geometry over combinatorial or nonfield structures

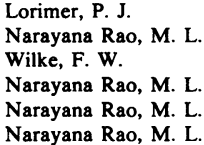

50D40 Line geometry

50D45 Circle and sphere geometry: Lie Laguerre, Möbius

50D50 Geometries of other space elements

50D99 None of the above, but in this section

\section{2-XX CONVEX SETS AND GEOMETRIC INEQUALITIES}

$52-00$ Difficult to classify at the second level (must also be assigned at least one other classification number in this section)

52-01 Elementary exposition (collegiate level)

52-02 Advanced exposition (research surveys, etc.)

$52-03$ Historical
52-04 Explicit machine computation and programs (not the theory of computation or programming)

52A05 Convex sets without dimension restrictions

$$
\text { Kenelly, John W., Jr. 2P }
$$

52A10 Convex sets in 2 dimensions

$\begin{array}{ll}\text { Chui, Charles Kam-tai } & 1 \mathrm{P} \\ \text { Stavrakas, Nick M. } & 1 \mathrm{P} \\ \text { Hare, William R. } & 3 \mathrm{P} \\ \text { Sparks, Arthur G. } & 2 \mathrm{P}\end{array}$

52A15 Convex sets in 3 dimensions

52A20 Convex sets in $\mathrm{n}$ dimensions

$\begin{array}{ll}\text { Hansen, Wolfhard } & \text { 1P } \\ \text { Kruskal, Joseph B. } & \text { 1P } \\ \text { Buchman, E. O. } & \text { 1P } \\ \text { Petty, C. M. } & \text { 1P } \\ \text { Butler, G. J. } & \text { IP }\end{array}$

52A25 Convex polyhedra

$\begin{array}{ll}\text { Hoffman, Alan J. } & \text { 1P } \\ \text { Walkup, David W. } & \text { 1P } \\ \text { Yeh, R. Z. } & \text { 1S } \\ \text { Banchoff, Thomas F. } & \text { iS } \\ \text { Petty, C. M. } & \text { is }\end{array}$

52A30 Star-shaped sets

Hare, William R.

Evans, B. D.

Ludescher, W. $\mathbf{H}$.

Kenelly, John W., Jr.

Halpern, Benjamin R.

Sparks, Arthur G.

Buchman, E. O.

$P$
$P$
$P$
$P$
$P$

52A35 Helly-type theorems

Hansen, Wolfhard

52A40 Inequalities and extremum problems

Butler, G. J.

$1 \mathrm{P}$

52A45 Packing and covering [See also 05B40, 10E30, 50B30.]

Schmidt, Wolfgang $M$.

$1 \mathrm{P}$

52A50 Hilbert geometry and other distance geometries

Petty, C. M.

52A55 Spherical convexity [See also 50C20.]

53-XX DIFFERENTIAL

GEOMETRY \{For differential topology, see 57Dxx. For foundational questions of differentiable manifolds, see 58Axx. ? 
53-00 Difficult to classify at the second level (must also be assigned at least one other classification number in this section)

53-01 Elementary exposition (collegiate level)

53-02 Advanced exposition (research surveys, etc.)

53-03 Historical

53-04 Explicit machine computation and programs (not the theory of computation or programming)

\section{Axx Classical differential geometry}

53A05 Curves and surfaces in euclidean space

Gottlieb, David

Breuer, Shlomo

Chen, Bang-yen

Chen, Bang-yen

1P

$2 \mathrm{P}$

$4 S$

53A10 Minimal surfaces

Reilly, Robert C.

Geveci, Tunc

Ruh, Ernst Alfred

Chen, Bang-yen

53A15 Affine differential geometry

53A20 Projective differential geometry

53A25 Differential line geometry

53A30 Conformal differential geometry

Kenelly, John W., Jr.

$2 \mathrm{P}$

53A35 Noneuclidean differential geometry

Fulton, Curtis M

Fillmore, Jay P.

53A40 Other special differential geometries

53A45 Vector and tensor analysis

53A50 Spinor analysis

53A55 Differential invariants (local

theory), geometric objects

53A99 None of the above, but in this section

53Bxx Local differential geometry

53B05 Affine connections

53B10 Projective connections

53B15 Other connections

53B20 Local Riemannian geometry

Blair, David E.

$1 \mathrm{P}$

$\begin{array}{lr}\text { Rosenthal, Aaron } & \text { 1P } \\ \text { Glasner, Moses } & \text { IS } \\ \text { Chow, Kwang-nan } & \text { 1S } \\ \text { Karcher, Hermann } & \text { 1P } \\ \text { Sen, Rabindra Nath } & \text { 1P } \\ \text { Ruh, Ernst Alfred } & \text { iS } \\ 5 \text { Submanifolds } & \\ \text { Lancaster, G. M. } & \text { 1P } \\ \text { Chen, Bang-yen } & \text { 4S }\end{array}$

53B30 Lorentz metrics, indefinite metrics

53B35 Hermitian and Kählerian structures [See also 32Cxx.]

53B40 Finsler spaces and generalizations (areal metrics)

53B99 None of the above, but in this section

53Cxx Global differential geometry

\{For related bundle theory, see 55Fxx, 57Dxx.

53C05 Connectionsi

53C15 Almost complex, contact, symplectic, almost product structures, etc. Closs, Mike $\mathbf{P}$.

$1 \mathrm{P}$

53C20 Riemannian manifolds, including pinching [See also 58B20.]

Rosenthal, Aaron
Tsagas, Grigorios
Byers, William P.
Wallach, Nolan R.
Warner, Frank W.
Weinstein, Alan D.
Glasner, Moses
Chow, Kwang-nan
Karcher, Hermann
Chen, Bang-yen
Ruh, Ernst Alfred

53C25 Special Riemannian manifolds (Einstein, Sasakian, etc.)

53C30 Homogeneous manifolds [See also 57F15.]

Closs, Mike P.

Weinstein, Alan D.

53C35 Symmetric spaces [See also 57F15.]

Conlon, Lawrence

Tirao, Juan A.

53C40 Submanifolds, isometric imbeddings [See also 57D40.]

Reilly, Robert C.

Chen, Bang-yen

Chen, Bang-yen

Ruh, Ernst Alfred

Banchoff, Thomas F.

$1 P$
$2 P$
$3 P$
$1 P$
$1 P$


Chen, Bang-yen $4 \mathbf{P}$

$53 \mathrm{C} 45$ Global surface theory (convex surfaces à la A. D. Aleksandrov)

Banchoff, Thomas $F$.

IP

53C50 Lorentz manifolds, manifolds with indefinite metrics

53C55 Hermitian and Kählerian structures [See also 32 Cxx.]

53C60 Finsler spaces and generalizations (areal metrics) [See also 58B20.]

MacLachlan, C.

53C65 Integral geometry, differential forms, currents, etc.

Chen, Bang-yen

53C70 Direct methods (G-spaces of Busemann, etc.)

$53 C 75$ Geometric orders, order geometry

53 C99 None of the above, but in this section

\section{4-XX GENERAL}

TOPOLOGY \{For the topology of manifolds of all dimensions, see 57Axx. ]

54-00 Difficult to classify at the second level (must also be assigned at least one other classification number in this section)

54-01 Elementary exposition (collegiate level)

54-02 Advanced exposition (research surveys, etc.)

54-03 Historical

54-04 Explicit machine computation and programs (not the theory of computation or programming)

\section{Axx Generalities}

54A05 Topological spaces and generalizations (closure spaces, etc.)

$\begin{array}{ll}\text { Kleitman, Daniel J. } & 2 S \\ \text { Richardson, G. D. } & 2 S \\ \text { Sikkema, Carl D. } & 1 S \\ \text { Anderson, Bruce A. } & 2 P\end{array}$

54A10 Change of topology, comparison of topologies

Lee, Yu-lee

Stecker, G. E.

Anderson, Bruce A.

Stewart, D. G.

Anderson, Bruce A
54A15 Syntopogeneous structures

54A20 Convergence (general theory) and limits

$\begin{array}{ll}\text { Wilbur, W. John } & \text { 1P } \\ \text { Pfeffer, W. } & \text { IP } \\ \text { Pettis, B. J. } & \text { 1P } \\ \text { Liu, Chen-tung } & \text { 2P } \\ \text { Priestley, W. M. } & \text { 1S } \\ \text { Richardson, G. D. } & \text { 1P } \\ \text { Richardson, G. D. } & \text { 2P } \\ \text { Wayment, S. G. } & \text { IP } \\ \text { Edwards, J. R. } & \text { IP } \\ \text { Richardson, G. D. } & 3 \text { P } \\ \text { Wyler, Oswald } & \text { PP }\end{array}$

54A25 Cardinality properties (discrete subsets, weight, etc.)

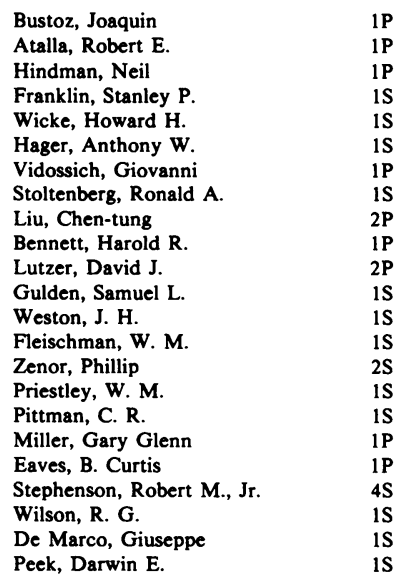
54A99 None of the above, but in this section

Comfort, W. W.

$1 \mathrm{P}$

\section{Bxx Basic constructions}

54B05 Subspaces

Peek, Darwin E.

54B10 Product spaces

West, James E.

Noble, Norman

Hagopian, Charles L.

Priestley, W. M.

Young, W. L.

Berney, E. S.

Herrlich, Horst

Stephenson, Robert M., Jr.

Michael, Ernest A.

Kost, Frank

Steiner, A. K.

Zenor, Phillip

Peek, Darwin E.

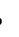

54B15 Quotient spaces, decompositions Kozlowski, George 
Whyburn, Gordon Thomas

Collins, P. J.

Williams, G. Kenneth

Hinrichsen, J. W.

Fitzpatrick, Ben, Jr.

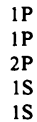

54B17 Adjunction spaces and similar constructions

Borges, Carlos J. R.

2P

54B20 Hyperspaces

Keesling, James Edgar

Smithson, Raymond E

Henderson, George W.

Rogers, James Ted, Jr.

54B25 Sums, inverse limits

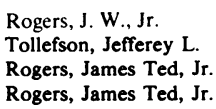

$1 \mathrm{P}$
1P
$2 \mathrm{P}$
iS
iS

99 None of the above, but in this section

Zame, Alan
Cobb, John

Zenor, Phillip

54Cxx Maps and general types of spaces defined by maps

54C05 Continuous maps

Cain, George L., Jr.
Lambert, H. W.
Rushing, T. B.
Bedford, Eric
Edwards, J. R.
Janoš, Ludvík
Hager, Anthony W.
McCarty, G. S., Jr.
Geroch, Robert P.
Kronheimer, E. H.
Wyler, Oswald
Bellamy, David P.

Bellamy, David P.

54C10 Special maps: open, closed, perfect, almost open, light, etc.

Atalla, Robert E.

Bustoz, Joaquin

Kozlowski, George

Hyman, D. M

Long, Paul E.

Willard, Stephen

Janoš, Ludvík

Wicke, Howard $\mathrm{H}$

Yohe, J. M.

Noble, Norman

Glaser, Leslie C.

Stoltenberg, Ronald A

Cain, George L., Jr.

Sehgal, V. M.

McGehee, Earl E., Jr.

Long, Paul E.

Whyburn, Gordon Thomas

Bredon, Glen E.

Rogers, J. W., Jr.

$\begin{array}{ll}\text { Hagan, Melvin R. } & \text { IP } \\ \text { Collins, P. J. } & \text { IP } \\ \text { Hager, Anthony W. } & \text { 2S } \\ \text { Williams, G. Kenneth } & 2 \mathrm{P} \\ \text { Jaworowski, Jan W. } & 1 \mathrm{P} \\ \text { Michael, Ernest A. } & 1 \mathrm{P} \\ \text { Keesling, James Edgar } & 2 \mathrm{~S} \\ \text { Steiner, A. K. } & 2 \mathrm{~S} \\ \text { Hagan, Melvin R. } & 2 \mathrm{P} \\ \text { Peek, Darwin E. } & 1 \mathrm{~S} \\ \text { Bennett, Harold R. } & 4 \mathrm{P} \\ \text { Berney, E. S. } & 2 \mathrm{P} \\ \text { Keesling, James Edgar } & 4 \mathrm{P}\end{array}$

54C15 Retraction

Atalla, Robert E.

Bustoz, Joaquin

Hager, Anthony W.

Bellamy, David P.

Ellis, Robert L.

$1 \mathrm{P}$
$1 \mathrm{P}$
$2 \mathrm{~S}$
$2 \mathrm{P}$
$1 \mathrm{P}$
$1 \mathrm{P}$
$2 \mathrm{~S}$
$2 \mathrm{~S}$
$2 \mathrm{P}$
$1 \mathrm{~S}$
$4 \mathrm{P}$
$2 \mathrm{P}$
$4 \mathrm{P}$

54C20 Extension

$\begin{array}{ll}\text { Transue, William R. R. } & 1 \mathrm{P} \\ \text { Bennett, Ralph } & 1 \mathrm{P} \\ \text { Wong, Raymond Y. T. } & 2 \mathrm{P} \\ \text { Mattson, Don A. } & 1 \mathrm{~S} \\ \text { Ellis, Robert L. } & 1 \mathrm{P}\end{array}$

54C25 Imbedding

$\begin{array}{ll}\text { Lister, F. M. } & \text { 1S } \\ \text { Wayment, S. G. } & \text { 1P } \\ \text { Summerhill, Ralph R. } & \text { 1P } \\ \text { Edwards, J. R. } & \text { 1P } \\ \text { Chew, Kim-peu } & \text { 1P } \\ \text { Henderson, George W. } & \text { 1P } \\ \text { Creede, Geoffrey D. } & \text { 1P } \\ \text { Kost, Frank } & \text { 1S }\end{array}$

54 C30 Real-valued functions [See also 26-XX.]

Noble, Norman

Smithson, Raymond E.

Geroch, Robert P.

McCarty, G. S., Jr.

Kronheimer, E. H.

Peek, Darwin E.

54C35 Function spaces [See also 58D15.]

$\begin{array}{ll}\text { Vidossich, Giovanni } & \text { 1P } \\ \text { Wayment, S. G. } & \text { 1S } \\ \text { O'Meara, Paul } & 1 \mathrm{P} \\ \text { Keesling, James Edgar } & 3 \mathrm{~S}\end{array}$

54C40 C(X); algebraic techniques (ideals, etc.) [See also 46J10.]

Jensen, G. A.

Hager, Anthony W.

Foguel, Shaul Reuven

Hochster, M.

Wilson, R. G

De Marco, Giuseppe

Orsatti, Adalberto

De Marco, Giuseppe

$1 \mathrm{P}$
$2 \mathrm{P}$
$1 \mathrm{P}$
$1 \mathrm{P}$
$1 \mathrm{P}$
$1 \mathrm{P}$

54C45 C-and $\mathrm{C}^{*}$-imbedding

Mattson, Don A. 
54C50 Zero sets, Baire sets and functions [See also 26A21.]

$\begin{array}{ll}\text { Willard, Stephen } & 1 \mathrm{~S} \\ \text { Erdös, Paul } & \text { IP } \\ \text { Stone, A. H. } & \text { IP } \\ \text { Kost, Frank } & 1 \mathrm{~S} \\ \text { Peek, Darwin E. } & 1 \mathrm{P}\end{array}$

54C55 Absolute neighborhood extensor, absolute extensor, absolute neighborhood retract (ANR), absolute retract spaces (general properties)

Bing, R. H.

Jaworowski, Jan W.

$1 \mathrm{P}$

$1 P$

54C60 Set-valued maps

Wicke, Howard $\mathrm{H}$.

Williams, Richard K.

Smithson, Raymond E.

Williams, Richard $\mathrm{K}$.

Gray, William J.

Smithson, Raymond E.

George, John $\mathrm{H}$

Sehgal, V. M.

Smithson, Raymond E

Wyler, Oswald

54C65 Selections

Nadler, Sam B., Jr.

Ward, L. E., Jr.

Smithson, Raymond E.

$54 C 99$ None of the above, but in this section

\section{Dxx Fairly general properties}

54D05 Connected and locally connected spaces (general aspects)

\begin{tabular}{ll} 
Kozlowski, George & $1 \mathrm{~S}$ \\
Unger, Gerald S. & $1 \mathrm{P}$ \\
Long, Paul E. & $1 \mathrm{~S}$ \\
Fraser, Robert B., Jr. & $1 \mathrm{~S}$ \\
Bacon, Philip & $1 \mathrm{P}$ \\
McGehee, Earl E., Jr. & $1 \mathrm{~S}$ \\
Long, Paul E. & $2 \mathrm{~S}$ \\
Wong, Yim-ming & $1 \mathrm{P}$ \\
Hagan, Melvin R. & $1 \mathrm{~S}$ \\
Miller, Gary Glenn & $1 \mathrm{P}$ \\
Breuer, Shlomo & $2 \mathrm{~S}$ \\
Jobe, John & $2 \mathrm{~S}$ \\
Crawford, Albert L. & $1 \mathrm{~S}$ \\
Hunt, John & $1 \mathrm{P}$ \\
García Máynez, Adalberto & $1 \mathrm{P}$ \\
Collins, P. J. & $1 \mathrm{P}$ \\
Williams, G. Kenneth & $2 \mathrm{~S}$ \\
Hagan, Melvin R. & $2 \mathrm{P}$ \\
\hline
\end{tabular}

54D10 Separation axioms, $T_{0}-T_{3}$

Stecker, G. E.

Loeb, Peter Adolf

Richardson, G. D.

Wong, Yim-ming

Miller, Gary Glenn

Breuer, Shlom

Hunt, John

García Máynez, Adalberto
Wyler, Oswald

1P

54D15 Higher separation axioms

(completely regular, normal, perfectly or collectionwise normal, etc.)

Landau, Martin
Steen, Lynn A.
Keesling, James Edgar
Fletcher, Peter
McCoy, R. A.
Slover, Rebecca Ellen
Wicke, Howard H.
Worrell, John M., Jr.
French, James A.
Wong, Yim-ming
Berney, E. S.
Bennett, Harold R.
Aull, C. E.
Zenor, Phillip

1P
1S
$1 \mathrm{~S}$
$1 \mathrm{P}$
$2 \mathrm{P}$
$1 \mathrm{P}$
$2 \mathrm{P}$
$1 \mathrm{P}$
$1 \mathrm{P}$
$1 \mathrm{P}$
$1 \mathrm{~S}$
$3 \mathrm{P}$
$1 \mathrm{P}$
$4 \mathrm{P}$

54D20 Covering properties: Lindelöf, (m,n)-compact, paracompact, pointwise paracompact, etc.

Ornstein, Donald $\mathrm{S}$.

Wicke, Howard $\mathrm{H}$.

Burke, Dennis K.

Lutzer, David J.

Bennett, Harold R

Gulden, Samuel L.

Weston, J. H.

Fleischman, W. M.

Zenor, Phillip

Keesling, James Edgar

Maharam, Dorothy

Kemperman, J. H. B.

Fletcher, Peter

McCoy, R. A.

Slover, Rebecca Ellen

Vidossich, Giovanni

Worrell, John M., Jr.

Wicke, Howard $\mathrm{H}$.

Hodel, Richard E.

Comfort, W. W.

Wong, Yim-ming

Berney, E. S.

Kullman, David E.

Vidossich, Giovanni

Frolík, Zdeněk

Tamano, Hisahiro

Vaughan, J. E.

Bennett, Harold R.

O'Meara, Paul

Aull, C. E.

Steiner, A. K.

Wilson, R. G

De Marco, Giuseppe

Zenor, Phillip

Bennett, Harold $\mathbf{R}$.

Berney, E. S.

Alexander, Charles C.

54D25 "P-minimal" and "P-closed" spaces

Stecker, G. E.

Liu, Chen-tung

Stephenson, Robert M., Jr.

Stephenson, Robert M. Jr.

Herrlich, Horst 
54D30 Compact spaces and generalizations

Willard, Stephen

Janoš, Ludvík

Franklin, Stanley P.

Foguel, Shaul Reuven

Stephenson, Robert M., Jr.

Weston, J. H.

Gulden, Samuel L.

Fleischman, W. M.

Richardson, G. D.

Zenor, Phillip

Janoš, Ludvík

Vidossich, Giovanni

Hager, Anthony W.

Stephenson, Robert M., Jr.

Creede, Geoffrey D.

54D35 Compactifications, etc.

Hindman, Neil

Stecker, G. E.

Zame, Alan

Liu, Chen-tung

Loeb, Peter Adolf

Cain, George L., Jr.

Biles, Charles M.

Richardson, G. D

Wong, Raymond Y. T.

Steiner, Eugene F.

Steiner, A. K.

Comfort, W. W.

Atalla, Robert E

Kullman, David E.

Vidossich, Giovann

Hansell, Roger W.

Hager, Anthony W.

Stephenson, Robert M., Jr.

Okuyama, Akihiro

Bennett, Harold R.

Creede, Geoffrey D.

Kost, Frank

Keesling, James Edgar

54D40 Remainders

Hindman, Neil

Steiner, Eugene F.

Steiner, A. K

Okuyama, Akihiro

54D45 Local compactness, $\sigma$-compactness

McGehee, Earl E., Jr.

Long, Paul E.

Steiner, A. K

Steiner, Eugene F.

Worrell, John M., Jr.

Wicke, Howard $\mathrm{H}$.

Wong, Yim-ming

Keesling, James Edgar

$54 \mathrm{D} 50 \mathrm{k}$-spaces

Bagley, Robert W.

Weddington, D. D.

Sieradski, Allan J.

Whyburn, Gordon Thomas

54D55 Sequential spaces

Franklin, Stanley P.
Whyburn, Gordon Thomas

1S

54D60 Realcompactness and realcompactification

$\begin{array}{ll}\text { Liu, Chen-tung } & \text { 1S } \\ \text { Liu, Chen-tung } & \text { 2S } \\ \text { Zenor, Phillip } & \text { 3P } \\ \text { Chew, Kim-peu } & 1 P \\ \text { Wilson, R. G. } & 1 P \\ \text { De Marco, Giuseppe } & 1 P\end{array}$

54D99 None of the above, but in this section

\section{Exx Spaces with richer structures}

54E05 Proximity structures and generalizations

Mattson, Don A

$1 \mathrm{P}$

54E10 p-maps

Mattson, Don A.

$1 \mathrm{P}$

54E15 Uniform structures and generalizations

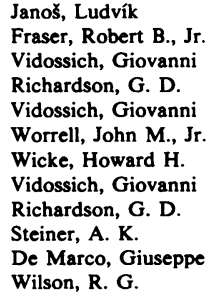

P

54E20 Stratifiable spaces, cosmic spaces, etc.

Stoltenberg, Ronald A.

Kullman, David E.

Tamano, Hisahiro

Vaughan, J. E.

54E25 Semimetric spaces

Miller, Gary Glenn

Berney, E. S.

54E30 Moore spaces

Kullman, David E.

Crawford, Albert L.

Jobe, John

Creede, Geoffrey D.

Aull, C. E.

Berney, E. S.

Bennett, Harold $\mathbf{R}$.

Alexander, Charles C

54E35 Metric spaces, metrizability

Hyman, D. M.

Willard, Stephen

Ornstein, Donald S.

Engelking, Ryszard

Wong, Raymond Y. T.

Fraser, Robert B., Jr.

Lutzer, David J.

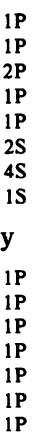


Nichols, J. C.

Cutler, William H.

Piech, M. Ann

Gelbaum, Bernard R.

Oxtoby, John C.

Borges, Carlos J. R.

Prosser, Reese T.

Edwards, J. R.

Kullman, David E.

Smithson, Raymond E.

Crawford, Albert L.

Jobe, John

Hansell, Roger W

Isbell, John R.

Tamano, Hisahiro

Vaughan, J. E.

Janoš, Ludvík

Bennett, Harold $\mathbf{R}$.

Sehgal, V. M.

George, John H.

Smithson, Raymond E.

O'Meara, Pau

Aull, C. E.

Fournier, Gilles

Peek, Darwin E.

Alexander, Charles C.

Ellis, Robert L.

54E40 Isometries, contractions, expansions

Janoš, Ludvík

Williams, Richard $\mathrm{K}$.

Sehgal, V. M.

Williams, Richard $\mathbf{K}$.

Isbell, John R.

Fournier, Gilles

54E45 Compact (locally compact) metric spaces

Janoš, Ludvík

Zame, Alan

Hagopian, Charles L.

Prosser, Reese T.

Collins, P. J.

Janoš, Ludvík

Smithson, Raymond E.

George, John H

Sehgal, V. M.

54E50 Complete metric spaces

Bustoz, Joaquin

Atalla, Robert E.

Sehgal, V. M

Bookhout, Glenn A

Edwards, J. R.

Peek, Darwin E.

54E55 Bitopologies

54E60 CW-complexes, triangulable spaces

Borges, Carlos J. R.

$2 S$

54E99 None of the above, but in this section

Bacon, Philip

$1 P$

\section{Fxx Special properties}

54F05 Ordered topological spaces, partially ordered spaces [See also 06A45.]

$\begin{array}{ll}\text { Edmondson, Don E. } & \text { 1P } \\ \text { Lutzer, David J. } & \text { IP } \\ \text { Choe, Tae Ho } & 1 \mathrm{P} \\ \text { Lutzer, David J. } & 2 \mathrm{P} \\ \text { Bennett, Harold R. } & 1 \mathrm{P} \\ \text { Weston, J. H. } & 1 \mathrm{P} \\ \text { Gulden, Samuel L. } & 1 \mathrm{P} \\ \text { Fleischman, W. M. } & 1 \mathrm{P} \\ \text { Steen, Lynn A. } & \text { IP } \\ \text { Biles, Charles M. } & 1 \mathrm{~S} \\ \text { Madell, Robert L. } & 1 \mathrm{P} \\ \text { Madell, Robert L. } & 1 \mathrm{~S} \\ \text { Breuer, Shlomo } & 2 \mathrm{P} \\ \text { Smithson, Raymond E. } & 3 \mathrm{P} \\ \text { Bennett, Harold R. } & 3 \mathrm{P}\end{array}$

54F15 Continua and generalizations

Stephenson, Robert M., Jr. 3P

Glaser, Leslie C. IS

Hagopian, Charles L. IP

Wayment, S. G. $\quad 1 P$

Trotter, William T., Jr. IP

Summerhill, Ralph R. IS

Davis, Harvey S.

54F20 Special types of continua

Transue, William R. R.

Bennett, Ralph

Yohe, J. M.

Hagopian, Charles L.

Rogers, J. W., jr.

Trotter, William T., Jr.

Pittman, C. R.

Summerhill, Ralph R.

Crawford, Albert L.

Jobe, John

Henderson, George W.

Wilder, B .E.

Rogers, James Ted, Jr.

Fitzpatrick, Ben, Jr.

Hindrichsen, J. W.

Hagopian, Charles I.

Bellamy, David P.

Hagan, Melvin R.

54F25 Peano spaces and generalizations

Dickman, R. F., Jr.

Hagopian, Charles L.

Chewning, W. C.

54F30 Cyclic elements

54F35 Higher dimensional local connectedness [See also 55Bxx, 55Cxx.]

Eaton, William T.

$1 \mathrm{~S}$

54F40 Compact (locally compact) absolute neighborhood retracts

Brown, Robert F.

54F45 Dimension theory [See also $55 \mathrm{C} 10$.

Landau, Martin

Yohe, J. M.

Cobb, John

Choe, Tae Ho

Nichols, J. C.
$1 P$
$1 P$
$1 P$
$1 P$
$2 P$
$1 S$
$1 S$
$1 P$
$1 P$
$2 P$
$1 P$
$1 P$
$2 P$
$1 P$
$1 P$
$2 P$
$2 P$
$2 S$ 
Bookhout, Glenn A

McCoy, R. A.

Fletcher, Peter

Slover, Rebecca Ellen

French, James A.

Canfell, M. J.

Keesling, James Edgar

Keesling, James Edgar
Prosser, Reese T.

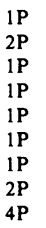

54F50 Spaces of dimension $\leq 1$; curves, dendrites [See also 26A03.]

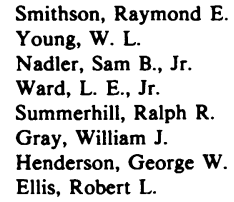

54F55 Unicoherence, multicoherence

Transue, William R. R.

Bennett, Ralph

Hagan, Melvin R.

Chewning, W. C.

54F60 Maps into $S_{n}$

Dugundji, John

Curtis, Morton L.

Summerhill, Ralph $\mathbf{R}$

54F65 Topological characterizations of particular spaces

$$
\begin{aligned}
& \text { Dickman, R. F., Jr. } \\
& \text { Breuer, Shlomo } \\
& \text { Bennett, Harold R. }
\end{aligned}
$$

54F99 None of the above, but in this section

Sharp, Henry S.

1S

\section{Gxx Peculiar spaces}

54G05 Extremally disconnected spaces, F-spaces, etc.

Biles, Charles M.

Hochster, $M$.

54G10 P-spaces

54G15 Pathological spaces

Miller, Gary Glenn

54G20 Counterexamples

Burke, Dennis K.

Borges, Carlos J. R.

Bookhout, Glenn A

Berney, E. S.

Herrlich, Horst

Rogers, James Ted, Jr.

Steiner, A. K.

Chewning, W. C.

54G99 None of the above, but in this section

Borges, Carlos J. R

\section{Hxx Connections with other structures, applications}

54H05 Descriptive set theory (topological aspects of Borel, analytic, projective, etc. sets) [See also 04A15, 26A21, 28A05.]

Willmott, R. C.
Comfort, W. W.
Darst, Richard B.
Darst, Richard B.
Sharp, Henry S.
Mansfield, Richard
Darst, Richard B.
Hansell, Roger W.
Frolík, Zdeněk

$54 \mathrm{H} 10$ Topological representations of algebraic systems [See also 22-XX.]

\section{Heinicke, A. G.}

Wilcox, Howard J.

is

54H15 Transformation groups and semigroups [See also 20Mxx, 22-XX, 57Exx.]

West, James E.
Roberson, Fred A.
Gray, William J.
Eisenberg, Murray
Reddy, William L.
Coven, Ethan M.
Petersen, K. E.
Hanson, T. H. McH.
Jones, Gary D.
Janoš, Ludvík
Kaul, S. K.
Tollefson, Jefferey L.
Rogers, James Ted, Jr.
Wong, Raymond Y. T.
Janoš, Ludvík
Wilder, B .E.

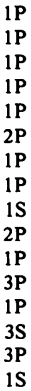

$54 \mathrm{H} 20$ Topological dynamics [See also 28A65, 34C35, 58Fxx.]

Roberson, Fred A.
Gray, William J.
Coven, Ethan M.
Eisenberg, Murray
Reddy, William L.
Coven, Ethan M.
Petersen, K. E.
O'Brien, Thomas
Markley, Nelson G.
Jones, Gary D.
Williams, Richard K.
Kaul, S. K.

1S

Gray, William J.

nberg, Murray

Coven, Ethan $\mathbf{M}$

Petersen, K. E.

Markley, Nelson

Williams, Richard K. theorems [See also $47 \mathrm{H} 10,55 \mathrm{C20}$.]

Janoš, Ludvík

West, James E.

Brown, Robert F.

Roberson, Fred A.

Gray, William J.

Smithson, Raymond E.

$1 \mathrm{P}$
$1 \mathrm{P}$
$1 \mathrm{P}$
$1 \mathrm{P}$
$1 \mathrm{P}$
$2 \mathrm{P}$
$1 \mathrm{P}$
$1 \mathrm{P}$
$1 \mathrm{~S}$
$2 \mathrm{P}$
$1 \mathrm{P}$
$3 \mathrm{P}$
$1 \mathrm{P}$
$3 \mathrm{~S}$
$3 \mathrm{P}$
$1 \mathrm{~S}$


Sehgal, V. M.

Young, W. L.

Brown, Robert F.

Yeh, R. Z.

Eaves, B. Curtis

Smithson, Raymond E.

Gray, William J.

Jaworowski, Jan W.

Smithson, Raymond E.

George, John $\mathbf{H}$

Sehgal, V. M.

Smithson, Raymond E.

Franks, R. L.

Marzec, R. P.

54H99 None of the above, but in this section

\section{J05 Nonstandard topology}

\section{5-XX ALGEBRAIC TOPOLOGY}

55-00 Difficult to classify at the second level (must also be assigned at least one other classification number in this section).

55-01 Elementary exposition (collegiate level)

55-02 Advanced exposition (research surveys, etc.)

$$
\text { Lam, Kee Yuen }
$$

$1 \mathrm{P}$

55-03 Historical

55-04 Explicit machine computation and programs (not the theory of computation or programming)

55Axx Low-dimensional topology \{See also 57Axx.

55A05 Fundamental group, presentations, free differential calculus [See also 20E40.]

Tollefson, Jefferey L.
Husch, Lawrence S.
Birman, Joan S.
Brown, Edward M.
Husch, Lawrence S.
Heil, Wolfgang
Byers, William P.
Gross, Jonathan L.
Heil, Wolfgang
Chen, Kuo-tsai
Feustel, C. D.
Murasugi, Kunio
Sumners, D. W.
Wood, John W.

55A10 Covering spaces, branched coverings

Jerrard, R. P.

Hamstrom, Mary-Elizabeth Brasher, Russell G.
Chen, Kuo-tsai

Feustel, C. D.

Holsztyński, W.

is

55A15 Graphs and map coloring [For other graph theory, see 05Cxx.]

Marx, Morris L.

Rosen, Ronald H.

Sher, R. B.

55A20 Two-dimensional complexes

Tondra, Richard J.

2P

55A25 Knots and links [For the high-dimensional case, see 57C45.]

$\begin{array}{ll}\text { Murasugi, Kunio } & \text { IP } \\ \text { Jerrard, R. P. } & \text { IP } \\ \text { Hamstrom, Mary-Elizabeth } & \text { IP } \\ \text { Goodrick, Richard E. } & \text { 1P } \\ \text { Heil, Wolfgang } & \text { 1P } \\ \text { Gross, Jonathan L. } & \text { 1P } \\ \text { Heil, Wolfgang } & \text { 2P } \\ \text { Murasugi, Kunio } & \text { 2P } \\ \text { Sumners, D. W. } & \text { 1P }\end{array}$

55A30 Wild knots and surfaces, etc

$\begin{array}{lr}\text { Burgess, C. E. } & \text { IS } \\ \text { Cannon, J. W. } & \text { IS } \\ \text { Loveland, L. D. } & \text { 1S } \\ \text { Loveland, L. D. } & \text { 2P } \\ \text { McPherson, James M. } & 1 \text { P } \\ \text { Tondra, Richard J. } & 3 P \\ \text { Sher, R. B. } & 2 S\end{array}$

55A35 Dehn's Lemma, sphere theorem, loop theorem, asphericity

Husch, Lawrence S.

Eaton, William T.

1S

55A40 Characterizations of $\mathrm{E}^{3}$ and $\mathrm{S}^{3}$ (Poincaré conjecture)

$\begin{array}{ll}\text { Tindell, Ralph S. } & 2 \mathrm{P} \\ \text { Loveland, L. D. } & 2 \mathrm{P} \\ \text { Lacher, R. C. } & \text { 1P } \\ \text { Costich, O. L. } & \text { iS } \\ \text { Galewski, D. E. } & \text { iS } \\ \text { Doyle, Patrick H. } & \text { iS }\end{array}$

55A99 None of the above, but in this section

$\begin{array}{ll}\text { Tondra, Richard J. } & \text { 2P } \\ \text { Feustel, C. D. } & 1 \text { P } \\ \text { Gross, Jonathan L. } & 2 P \\ \text { Lacher, R. C. } & 1 P \\ \text { Tondra, Richard J. } & \text { 3P } \\ \text { Wood, John W. } & 1 P\end{array}$

55Bxx Homology and cohomology theories

55B05 Čech types

Bacon, Philip

Brasher, Russell G.

$1 \mathrm{P}$

55B10 Singular theory

55B15 K-theory [For algebraic K-theory, 
see 18F25.]

Lazarov, Connor

Vasquez, Alphonse T

Thomas, C. B.

Smith, Larry

Landweber, Peter $S$ homology and cohomology spectra

$\begin{array}{ll}\text { Sieradski, Allan J. } & \text { 1P } \\ \text { Boardman, John } & \text { IP } \\ \text { Meyer, Jean-Pierre } & \text { 1P } \\ \text { Landweber, Peter S. } & \text { iP } \\ \text { Krueger, Warren M. } & \text { 1S }\end{array}$

55B25 Homology with local coefficients, equivariant cohomology

Brasher, Russell G.

Siegel, Jerrold

2P

55B30 Sheaf cohomology [See also $18 \mathrm{~F} 20,32 \mathrm{C} 35,32 \mathrm{~L} 10$.

Stoll, Wilhelm

55B35 Other homology theories

55B40 Axioms for homology theory and uniqueness theorems

55B45 Products and intersections

Lam, Kee Yuen

Chen, Kuo-tsai

$1 \mathrm{P}$

55B99 None of the above, but in this section

55Cxx Classical topics (For the topology of euclidean spaces and manifolds, see 57A05, 57A10,

57A15.

55C05 Duality

55C10 Dimension theory [See also 54F45.]

$$
\begin{aligned}
& \text { Jerrard, R. P. } \\
& \text { Hamstrom, Mary-Elizabeth } \\
& \text { Choe, Tae Ho }
\end{aligned}
$$

55C15 Absolute neighborhood retracts [See also 54C55.]

Jaworowski, Jan W.

55C20 Fixed points and coincidences [See also 54H25.]

Brown, Robert $F$.

Brown, Robert F.

Eaves, B. Curtis

Jaworowski, Jan W.

Huneke, John Philip

55C25 Degree

Holsztyński, W.

1S

55C30 Ljusternik-Schnirelman category of a space

Mielke, M. V.

$1 \mathbf{P}$

55C35 Finite groups of transformations (Smith theory)

$\begin{array}{ll}\text { Thomas, C. B. } & 1 \mathrm{P} \\ \text { Ku, Hsu-tung } & 2 \mathrm{P} \\ \text { Kwun, Kyung Whan } & 1 \mathrm{P} \\ \text { Bredon, Glen E. } & 2 \mathrm{~S} \\ \text { Bredon, Glen E. } & 3 \mathrm{P}\end{array}$

$55 C 99$ None of the above, but in this section

Hamstrom, Mary-Elizabeth

Jerrard, R. P.

Samelson, Hans

Brasher, Russell G.

Brasher, Russell G.

Kwun, Kyung Whan

Bredon, Glen E.

Bredon, Glen E.

$1 \mathrm{P}$
$2 \mathrm{P}$
$1 \mathrm{P}$
$2 \mathrm{~S}$
$3 \mathrm{P}$
th
$1 \mathrm{P}$
$1 \mathrm{P}$
$1 \mathrm{P}$
$1 \mathrm{P}$
$2 \mathrm{P}$
$1 \mathrm{P}$
$2 \mathrm{P}$
$4 \mathrm{P}$

55Dxx Homotopy theory (For simple homotopy type, see 57C10.\} 55D05 Homotopy extension properties, cofibrations

55D10 Homotopy equivalences

$\begin{array}{lr}\text { Rees, Elmer } & \text { 1S } \\ \text { Hall, Michael H. } & \text { 1S } \\ \text { Sieradski, Allan J. } & 2 P\end{array}$

55D15 Classification of homotopy type

Feustel, C. D.

Meyer, Jean-Pierre

Sieradski, Allan J.

James, Ioan $\mathbf{M}$

is

55D20 Eilenberg-MacLane spaces

Meyer, Jean-Pierre

55D25 Spanier-Whitehead duality

Boardman, John

55D30 Eckmann-Hilton duality

55D35 Loop spaces

Hubbuck, John

55D40 Suspensions

Haslam, Harold B.

$1 P$

55D45 $\mathrm{H}$-spaces and duals

Bacon, Philip

Brown, Robert F

Arkowitz, Martin

Brown, Robert F.

Haslam, Harold B.

Meyer, Jean-Pierre

Sieradski, Allan J.

Haslam, Harold B.

Hubbuck, John

Haslam, H. B

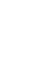


55D50 Category and cocategory, etc. 55D99 None of the above, but in this section

Curtis, Douglas W.

Rushing, T. B

Rees, Elmer

Boardman, John

Kahn, Donald W.

1S
$1 P$
$1 P$
$1 P$
$1 P$

\section{Exx Homotopy groups}

55E05 Homotopy groups, general; sets of homotopy classes

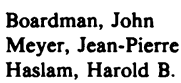

Haslam, Harold B.

$$
\begin{aligned}
& 1 S \\
& 1 P \\
& 2 P
\end{aligned}
$$

55E10 Stable homotopy groups

Segal, David M. Kahn, Donald W.

55E15 Whitehead products and generalizations

Haslam, Harold B.

James, Ioan $M$

55E20 Homotopy groups of wedges, joins, and simple spaces

Edelson, Allan L.

$1 \mathbf{P}$

55E25 Hopf invariants

55E30 Homotopy groups of triads, n-ads

55E35 Operations in homotopy groups

55E40 Homotopy groups of spheres

55E45 Stable homotopy of spheres

Kahn, Donald W.

Krueger, Warren $M$.

$1 \mathbf{P}$
$1 \mathrm{P}$

55E50 The J-morphism

55E55 Cohomotopy groups

55E99 None of the above, but in this section

Edelson, Allan L.

$1 \mathbf{P}$

55Fxx Fiber spaces and bundles

\{See also 18F15.\}

55F05 Fiber spaces

Unger, Gerald S.

Wright, Perrin

$1 P$

$1 \mathrm{~S}$

55F10 Fiber bundles

Gelbaum, Bernard R.

James, Ioan $\mathbf{M}$.

1P
$1 P$

55F15 Classification

Siegel, Jerrold
55F20 Spectral sequences and homology of fiber spaces [See also 55Hxx.]

James, Ioan $\mathbf{M}$.

$1 \mathrm{~S}$

55F25 Sphere bundles and vector space bundles

Mahowald, Mark E.
Gitler, Samuel
Milgram, R. James
Bredon, Glen E.
Riddell, R. C.
Smith, Larry
Glover, H. H.
Becker, James C.
Edelson, Allan L.
Sieradski, Allan J.
Wood, John W.

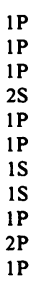

55F35 Classifying spaces of groups and H-spaces

Siegel, Jerrold

$1 \mathbf{P}$

55F40 Homology of classifying spaces, characteristic classes

$$
\begin{aligned}
& \text { Gitler, Samuel } \\
& \text { Mahowald, Mark E. } \\
& \text { Milgram, R. James } \\
& \text { Clough, Robert R. } \\
& \text { Smith, Larry } \\
& \text { Landweber, Peter S. }
\end{aligned}
$$

Hubbuck, John

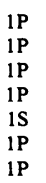

55F45 Homology and homotopy of $\mathrm{BO}$ and BU; Bott periodicity

55F50 Stable classes of vector space bundles, K-theory [For algebraic

K-theory, see 18F25.]

$\begin{array}{ll}\text { Smith, Larry } & \text { 1P } \\ \text { Edelson, Allan L. } & 1 \mathrm{P}\end{array}$

55F55 Fiberings with singularities

Tollefson, Jefferey L.

Antonelli, Peter L.

$1 \mathrm{P}$

55F60 Microbundles and block bundles

55 F65 Generalizations of fiber spaces and bundles

55F99 None of the above, but in this section

James, Ioan $\mathbf{M}$

$1 \mathbf{P}$

55Gxx Operations and obstructions

55G05 Primary cohomology operations

Krueger, Warren $M$.

55G10 Steenrod algebra

Mahowald, Mark E.

Gitler, Samuel

Milgram, R. James

Clough, Robert R.

Krueger, Warren $M$.

James, Ioan $M$.

Hubbuck, John
$1 \mathrm{P}$

1P
$1 P$
$1 P$
$1 P$
$1 P$
$1 P$
IP 
55G15 Symmetric products, cyclic products

55G20 Secondary and higher cohomology operations

Mahowald, Mark E.
Gitler, Samuel
Milgram, R. James
Kraines, David
Krueger, Warren M.
James, Ioan M.

Mitchell, George E.

Meyer, Jean-Pierre

$1 \mathrm{P}$

1P \{See also $18 \mathrm{Gxx}$.

55J05 Abstract complexes

55J10 Semisimplicial complexes

$55 \mathrm{~J} 15$ Chain complexes

55J20 Universal coefficient theorems, Bockstein operator

55G25 K-theory operations and generalized cohomology operations

55G30 Massey products

Kraines, David

55J25 Homology of a product, Künneth formula

55G35 Obstruction theory

55G36 Extension and compression of mappings

55G37 Classification of mappings

$55 \mathrm{G} 40$ Sectioning fiber spaces and bundles

Iwata, Kôichi

Becker, James C.

Glover, H. H.

James, Ioan $\mathbf{M}$

55G45 Postnikov systems, k-invariants

Mahowald, Mark E.

Gitler, Samue

Milgram, R. James

Kraines, David

Clough, Robert R.

Arkowitz, Martin

Haslam, Harold B.

Meyer, Jean-Pierre

Siegel, Jerrold

1S
1S
1S
1P
1P
IP
1P
1S
1P

55G99 None of the above, but in this section

\section{Hxx Spectral sequences}

55H05 General

55H10 Serre spectral sequences

Siegel, Jerrold

55H15 Adams spectral sequences

Segal, David M.

Krueger, Warren $M$.

Segal, David M.

55H20 Eilenberg-Moore spectral sequences

55H25 Generalized cohomology

$\begin{array}{ll}\text { Iwata, Kôichi } & 1 \mathrm{P} \\ \text { Meyer, Jean-Pierre } & 1 \mathrm{P} \\ \text { Landweber, Peter S. } & 1 \mathrm{P}\end{array}$

$55 \mathrm{H} 99$ None of the above, but in this section

\section{Meyer, Jean-Pierre \\ $1 \mathrm{P}$ \\ $55 \mathrm{~J} 30$ Duality \\ 55J99 None of the above, but in this} section

\section{7-XX MANIFOLDS AND CELL COMPLEXES \{For} complex manifolds, see $32 \mathrm{C} 10$. 57-00 Difficult to classify at the second level (must also be assigned at least one other classification number in this section)

$$
\begin{aligned}
& 57-01 \text { Elementary exposition } \\
& \text { (collegiate level) } \\
& \text { Samelson, Hans } \\
& 57-02 \text { Advanced exposition (research } \\
& \text { surveys, etc.) } \\
& 57-03 \text { Historical } \\
& 57-04 \text { Explicit machine computation } \\
& \text { and programs (not the theory of } \\
& \text { computation or programming) }
\end{aligned}
$$

\section{Axx Topological manifolds}

57A05 Topology of $E_{2}$, 2-manifolds

$\begin{array}{ll}\text { Dickman, R. F., Jr. } & \text { 1P } \\ \text { Transue, William R. R. } & \text { 1S } \\ \text { Bennett, Ralph } & \text { IS } \\ \text { Birman, Joan S. } & 1 \mathrm{P} \\ \text { Bacon, Philip } & 1 \mathrm{P} \\ \text { Brown, Edward M. } & 1 \mathrm{P} \\ \text { Tondra, Richard J. } & 1 \mathrm{P} \\ \text { Eaton, William T. } & 1 \mathrm{~S} \\ \text { O'Brien, Thomas } & 1 \mathrm{~S} \\ \text { Markley, Nelson G. } & 1 \mathrm{P} \\ \text { Wayment, S. G. } & 1 \mathrm{~S} \\ \text { Pittman, C. R. } & 1 \mathrm{P} \\ \text { Holsztyński, W. } & 1 \mathrm{P} \\ \text { Tondra, Richard J. } & 3 \mathrm{P} \\ \text { Wood, John W. } & 1 \mathrm{P} \\ \text { Rogers, James Ted, Jr. } & 2 \mathrm{~S} \\ \text { Hagopian, Charles L. } & 2 \mathrm{P}\end{array}$

57A10 Topology of $E_{3}, 3$-manifolds [See 
also 55Axx.]

Tollefson, Jefferey $\mathrm{L}$.

Husch, Lawrence $S$.

Lonergan, Francis D.

Bennett, Ralph

Transue, William R. R.

McMillan, D. R., Jr.

Cannon, J. W.

Burgess, C. E.

Eaton, William T

Daverman, Robert J.

Loveland, L. D.

Lambert, H. W.

Heil, Wolfgang

Downing, J. Scott

Gillman, David S.

Gross, Jonathan $\mathrm{L}$

Lister, F. M.

Wayment, S. G.

Sikkema, Carl D.

Tollefson, Jefferey L.

Loveland, L. D.

Gross, Jonathan L.

Henderson, George W.

McPherson, James $\mathbf{M}$.

Jaworowski, Jan W.

Costich, O. L.

Galewski, D. E.

Doyle, Patrick H.

Rogers, James Ted, Jr.

Chewning, W. C.

57A15 Topology of $E_{n}$, n-manifolds (3 $<\mathrm{n}<\infty$ )

Transue, William R. R.

Bennett, Ralph

Bing, R. H.

Brown, Robert F.

Bryant, John L.

Cobb, John

Choe, Tae Ho

Glaser, Leslie C.

Gillman, David S.

O'Brien, Thomas

Wayment, S. G.

Hanson, T. H. $\mathrm{McH}$

Rushing, T. B.

Sher, R. B.

Kaul, S. K.

Lacher, R. C.

Husch, Lawrence S.

Tollefson, Jefferey L.

Rogers, James Ted, Jr.

Jaworowski, Jan W.

Wood, John W.

Cantrell, James C.

Price, Thomas

Rushing, T. B.

Rogers, James Ted, Jr.

Sher, R. B.

57A17 Topology of topological vector spaces

Curtis, Douglas W.

Wong, Raymond Y. T.

Isbell, John R.

1P

$1 \mathrm{~S}$

57A20 Topology of infinite-dimensional manifolds [See also 58Bxx.]
Cutler, William H.

Wong, Raymond Y. T.

$1 \mathbf{P}$

57A30 Engulfing

Bryant, John L.

57A35 Imbeddings and immersions

$\begin{array}{ll}\text { Transue, William R. R. } & \text { 1S } \\ \text { Bennett, Ralph } & \text { 1S } \\ \text { McMillan, D. R., Jr. } & 1 \mathrm{P} \\ \text { Bryant, John L. } & 1 \mathrm{P} \\ \text { Rushing, T. B. } & 2 \mathrm{P} \\ \text { Loveland, L. D. } & 2 \mathrm{~S} \\ \text { Connelly, Robert } & 1 \mathrm{P} \\ \text { Lacher, R. C. } & 1 \mathrm{~S} \\ \text { Holsztyński, W. } & 1 \mathrm{P} \\ \text { Wood, John W. } & 1 \mathrm{P} \\ \text { Wright, Perrin } & 1 \mathrm{P}\end{array}$

57A40 Neighborhoods of submanifolds

57A45 Flatness

$\begin{array}{ll}\text { Rushing, T. B. } & \text { 2P } \\ \text { Gauld, David } & \text { IS } \\ \text { Cantrell, James C. } & \text { IP } \\ \text { Price, Thomas } & \text { 1P } \\ \text { Rushing, T. B. } & \text { 3P } \\ \text { Sher, R. B. } & \text { 2P }\end{array}$

57A50 $\mathrm{S}^{\mathrm{n}-1} \subset \mathrm{E}^{\mathrm{n}}$, Schoenflies problem

Loveland, L. D.

Loveland, L. D.

Gauld, David

Wright, Perrin

is

2P

is

57A55 Microbundles

57A60 Cellularity

Sher, R. B.

Lacher, R. C.

Sher, R. B.

$1 \mathrm{P}$

1S

57A65 Algebraic topology of manifolds

Brown, Robert F.

$3 \mathrm{~S}$

57A70 Cobordism

Rushing, T. B.

2P

57A99 None of the above, but in this section

Uchiyama, Saburô

Huneke, John Philip

Wright, Perrin

$1 \mathbf{P}$

2P

57Bxx Generalized manifolds \{See also $18 \mathrm{~F} 15$.

57B05 Local properties

Kozlowski, George

$1 \mathrm{P}$

57B10 Poincaré duality spaces

57B99 None of the above, but in this section

57Cxx PL-topology

57C05 The general topology of 
complexes

Cohen, Marshall $\mathrm{M}$.

Rees, Elmer

Sher, R. B.

57C10 Simple homotopy type,

Whitehead torsion, Reidemeister-Franz torsion, etc.

57C15 Triarigulating manifolds

Tindell, Ralph S.

57C20 Cobordism

Conrad, Bruce

57C25 Comparison of PL-structures: classification, Hauptvermutung

Tindell, Ralph S.

Conrad, Bruce

57C30 Engulfing

57C35 Imbeddings and immersions

Husch, Lawrence S.

Putz, $\mathbf{H}$.

Feustel, C. D.

Rees, Elmer

Connelly, Robert

Banchoff, Thomas F

57C40 Regular neighborhoods

$57 \mathrm{C} 45 \mathrm{Knots}$ and links (in high dimensions) [For the low-dimensional case, see 55A25.]

\section{Husch, Lawrence $S$.}

$2 S$

57C50 Microbundles and block bundles [See also 55F60.]

Putz, $\mathrm{H}$.

57C55 Approximations

Sher, R. B.

57 C99 None of the above, but in this section

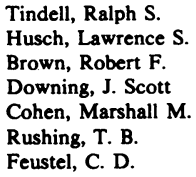

\section{Dxx Differential topology (For} foundational questions of differentiable manifolds, see 58Axx; for infinite dimensional manifolds, see $58 \mathrm{Bxx}$. 57D05 Triangulating 57D10 Smoothing
57D12 Smooth approximations

Rogers, James Ted, Jr.

57D15 Specialized structures on manifolds (spin manifolds, frame manifolds, etc.)

57D20 Characteristic classes

$\begin{array}{ll}\text { Mielke, M. V. } & \text { 1P } \\ \text { Ku, Hsu-tung } & 2 \text { P } \\ \text { Ku, Hsu-tung } & 4 P \\ \text { Smith, Larry } & 1 P \\ \text { Mitchell, George E. } & \text { 1S } \\ \text { Conrad, Bruce } & 1 P \\ \text { Segal, David M. } & 2 P\end{array}$

57D25 Vector fields, frame fields, etc.

Tsagas, Grigorios

Iwata, Kôichi

Bredon, Glen E.

57D30 Foliations

57D35 Differentiable mappings

57D40 Imbeddings and immersions

Samelson, Hans

Agoston, Max K.

Glover, H. H.

Becker, James C

Banchoff, Thomas F.

Halpern, Benjamin R.

57D45 Singularities of differentiable mappings

57D50 Diffeomorphisms

Robertson, Jack M.

$1 \mathrm{P}$

57D55 Differentiable structures

57D60 Homotopy spheres, Poincaré conjecture

$$
\begin{aligned}
& \text { Ku, Mei-chin } \\
& \text { Ku, Hsu-tung } \\
& \text { Ku, Hsu-tung } \\
& \text { Rosen, Ronald H. } \\
& \text { Agoston, Max K. } \\
& \text { Ku, Hsu-tung } \\
& \text { Conrad, Bruce } \\
& \text { Brender, Allan } \\
& \text { Schultz, Reinhard E. }
\end{aligned}
$$

57D65 Surgery and handlebodies

Mielke, M. V.

Downing, J. Scott

57D70 Critical points and critical submanifolds

57D75 O- and SO-cobordism

Mielke, M. V. Mitchell, George E.

57D80 h- and s-cobordism

Husch, Lawrence S.

$2 P$

57D85 Equivariant cobordism 
57D90 Other types of cobordism

Thomas, C. B.

Ku, Hsu-tung

Landweber, Peter S.

Brender, Allan

Segal, David M.

57D95 Realizing cycles by submanifolds

57D99 None of the above, but in this section

Samelson, Hans

Glover, H. H.

Becker, James C.

1P

$1 \mathrm{P}$

57Exx Topological transformation groups \{See also 20E40, 22-XX, 54H15, 58D05.

57E05 Topological properties of groups of homeomorphisms

Robertson, Jack M.

Keesling, James Edgar

Wright, Perrin

$1 P$
$3 P$
$1 P$

57E10 Compact groups of homeomorphisms

Tollefson, Jefferey L.

Conrad, Bruce

Franklin, Lawrence $\mathbf{M}$

Keesling, James Edgar

57 E15 Compact Lie groups of differentiable transformations

$\mathrm{Ku}$, Mei-chin
$\mathrm{Ku}$, Hsu-tung
$\mathrm{Ku}$, Hsu-tung
$\mathrm{Ku}$, Hsu-tung
Bredon, Glen E.
Bredon, Glen E.
$\mathrm{Ku}$, Hsu-tung
Conrad, Bruce
Bredon, Glen E.
Schultz, Reinhard E.

57E20 Noncompact Lie groups of transformations

57E25 Groups acting on specific manifolds

Bredon, Glen E.

Robertson, Jack M

O'Brien, Thomas

Bredon, Glen E.

$\mathrm{Ku}$, Hsu-tung

Jones, Gary D.

Conrad, Bruce

Schultz, Reinhard E

57E30 Discontinuous groups of transformations

Tollefson, Jefferey $\mathrm{L}$.

Husch, Lawrence $S$.

Mumford, David

2P

3P

57E99 None of the above, but in this section

Hanson, T. H. McH.

Conrad, Bruce

$1 \mathrm{P}$

57Fxx Homology and homotopy of topological groups and related structures

57F05 Hopf algebras [See also 16A24.]

$\begin{array}{ll}\text { May, J. Peter } & \text { is } \\ \text { Hubbuck, John } & \text { is }\end{array}$

57F10 Homology of Lie groups

57F15 Homology of homogeneous spaces of Lie groups

$\begin{array}{ll}\text { Milgram, R. James } & \text { 1S } \\ \text { Gitler, Samuel } & \text { 1S } \\ \text { Mahowald, Mark E. } & 1 S \\ \text { Lam, Kee Yuen } & 1 \mathrm{P} \\ \text { Segal, David M. } & 1 \mathrm{P} \\ \text { James, Ioan M. } & 1 S\end{array}$

57F20 Homotopy groups of topological groups and homogeneous spaces

$\begin{array}{ll}\text { Agoston, Max K. } & \text { 1P } \\ \text { Segal, David M. } & \text { 1P } \\ \text { James, Ioan M. } & \text { IP }\end{array}$

57F25 Homology of H-spaces

57F30 Bar and cobar constructions

57F35 Applications of Eilenberg-Moore spectral sequences [See also 55F20, $55 \mathrm{H} 20$.

57F99 None of the above, but in this section

Rees, Elmer

1S

58-XX GLOBAL ANALYSIS, ANALYSIS ON MANIFOLDS

\{For real-analytic and complex manifolds, see $32 \mathrm{Cxx}$; for geometric integration theory, see 49F20, 49F22.

58-00 Difficult to classify at the second level (must also be assigned at least one other classification number in this section)

$58-01$ Elementary exposition (collegiate level)

58-02 Advanced exposition (research surveys, etc.)

58-03 Historical

58-04 Explicit machine computation and programs (not the theory of computation or programming) 
58Axx General theory of differentiable manifolds

58A05 Differentiable manifolds, foundations

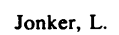

58A10 Differential forms Warner, Frank W.
Wallach, Nolan R. Jonker, L.

58A15 Exterior differential systems (Cartan theory) [For variational problems, see 49F05.]

58A20 Jets

58A25 Currents

58A30 Vector distributions (sub-bundles of the tangent bundles)

$$
\text { Bredon, Glen E. }
$$

58A99 None of the above, but in this section

$$
\text { Riddell, R. C. }
$$$$
1 \mathrm{P}
$$

\section{$58 \mathrm{Bxx}$ Infinite-dimensional} manifolds \{See also 57A20.\} 58B05 Homotopy and topological questions

$$
\begin{aligned}
& \text { Cutler, William H. } \\
& \text { Anderson, R. D. } \\
& \text { Curtis, Douglas W. }
\end{aligned}
$$

58B10 Differentiable and holomorphic questions

$$
\begin{aligned}
& \text { Riddell, R. C. } \\
& \text { Goodman, Victor }
\end{aligned}
$$

58B15 Fredholm structures

58B20 Riemannian, Finsler, and other geometric structures

$$
\text { Cutler, William H. }
$$

$1 \mathrm{P}$

58B99 None of the above, but in this section

\section{Cxx Calculus on manifolds}

$58 \mathrm{C} 05$ Real-valued functions

$58 \mathrm{C} 10$ Holomorphic maps

$$
\text { Greenfield, Stephen J. }
$$

$58 \mathrm{C} 15$ Implicit function theorems 58C20 Differentiation theory (Gâteaux, Fréchet, etc.)

$$
\text { Goodman, Victor }
$$

$58 C 25$ Differentiable maps and singularities
58C99 None of the above, but in this section

58Dxx Spaces and manifolds of mappings

58D05 Groups of diffeomorphisms and homeomorphisms as manifolds [See also 22Exx, 57E05.]

58D10 Spaces of imbeddings and immersions

58D15 Manifolds of mappings [See also 54C35.]

$$
\text { Riddell, R. C. }
$$

58D99 None of the above, but in this section

$$
\text { Robertson, Jack M. IP }
$$

58Exx Critical point theory (For the finite-dimensional case, see 57D70; for variational problems, see 49F15.

58E05 Abstract theory (Morse theory, Ljusternik-Schnirelman theory, etc.)

$$
\text { Kalmbach, Gudrun IP }
$$

$58 \mathrm{E} 10$ Application to the theory of geodesics

58E15 Application to extremal problems in several variables

58E99 None of the above, but in this section

58Fxx Differentiable dynamical systems (For abstract and symbolical topological dynamics, see 28A65, 34C35, 54H20.

58F05 Hamiltonian and other special systems

58F10 Stability theory

$\begin{array}{ll}\text { Williams, Robert F. } & \text { 1P } \\ \text { Shub, Michael } & \text { 1P } \\ \text { Hájek, Otomar } & \text { 1S }\end{array}$

58F15 Hyperbolic structures (expanding maps, Anosov systems, etc.)

58F20 Periodic points and zeta functions 58F99 None of the above, but in this section

58Gxx Differential operators on manifolds 
58G05 Elliptic complexes [See also $35 \mathrm{~N} 15$.]

58 G10 Index theorems and fixed point theorems

58G15 Pseudodifferential operators on manifolds

Petersen, Bent E.

$1 \mathbf{P}$

58G99 None of the above, but in this section

\section{H05 Pseudogroups}

(infinite-dimensional Lie groups) and deformations of structures (See also 22E65.

\section{0-XX PROBABILITY}

THEORY AND STOCHASTIC PROCESSES (For additional applications, see $10 \mathrm{Kxx}, 42 \mathrm{~A} 36$, $62-\mathrm{XX}, 90-\mathrm{XX}, 92-\mathrm{XX}, 93$ $-\mathrm{XX}, 94-\mathrm{XX}$.

60-00 Difficult to classify at the second level (must also be assigned at least one other classification number in this section)

60-01 Elementary exposition (collegiate level)

60-02 Advanced exposition (research surveys, etc.)

60-03 Historical

60-04 Explicit machine computation and programs (not the theory of computation or programming)

\section{A05 Axioms, foundations}

Brunk, Hugh D. Joffe, A. D.

60Bxx Probability theory on algebraic and topological structures 60B05 Probability measures on topological spaces

$$
\begin{aligned}
& \text { Loynes, R. M. } \\
& \text { Jain, N. C. } \\
& \text { Tserpes, Nicholas } \\
& \text { Mukherjea, Arunava }
\end{aligned}
$$

60B10 Convergence of probability measures, invariance theorems [See also 28A65.]

$$
\text { Loynes, R. M. }
$$
Huff, B. W.
60B15 Probability measures on groups, Fourier transforms, factorization

$\begin{array}{ll}\text { Tata, Mahabanoo N. } & \text { 1S } \\ \text { Staudte, Robert G., Jr. } & \text { 1S } \\ \text { Tserpes, Nicholas } & \text { 1P } \\ \text { Mukherjea, Arunava } & \text { 1P } \\ \text { Joffe, A. D. } & \text { 1S }\end{array}$

60B99 None of the above, but in this section

$60 C 05$ Combinatorial probability

Strait, Peggy Tang

$1 \mathrm{~S}$

O'Neil, Patrick Eugene $2 \mathrm{~S}$

60D05 Geometric probability

60E05 Distributions, characteristic functions

$\begin{array}{ll}\text { Andersen, G. R. } & \text { 1S } \\ \text { Severo, Norman C. } & \text { 1S } \\ \text { Slivka, John } & \text { 1S } \\ \text { Tata, Mahabanoo N. } & \text { IP } \\ \text { Staudte, Robert G., Jr. } & \text { 1P } \\ \text { Huff, B. W. } & \text { IP } \\ \text { Das, Minaketan } & \text { IS } \\ \text { Brown, B. M. } & \text { 1S } \\ \text { Athreya, Krishna Balasundaram } & \text { IP }\end{array}$

60Fxx Limit theorems

60F05 Central limit and other weak theorems

$\begin{array}{ll}\text { Severo, Norman C. } & \text { is } \\ \text { Slivka, John } & \text { is } \\ \text { Loynes, R. M. } & \text { is }\end{array}$

60F10 Large deviations

$\begin{array}{ll}\text { Pinsky, Mark A. } & \text { IP } \\ \text { Andersen, G. R. } & \text { 1P } \\ \text { Severo, Norman C. } & \text { 1P } \\ \text { Slivka, John } & \text { 1P }\end{array}$

60F15 Strong theorems [See also 28A65.]

$\begin{array}{ll}\text { Heyde, C. C. } & \text { 1P } \\ \text { Strait, Peggy Tang } & \text { 1P } \\ \text { Severo, Norman C. } & 1 \mathrm{P} \\ \text { Slivka, John } & 1 \mathrm{P} \\ \text { Jain, N. C. } & 1 \mathrm{P} \\ \text { Stackelberg, Olaf P. } & 1 \mathrm{P} \\ \text { Brown, B. M. } & 1 \mathrm{P} \\ \text { Athreya, Krishna Balasundaram } & 1 \mathrm{~S}\end{array}$

60F20 Zero-one laws

$\begin{array}{lr}\text { Severo, Norman C. } & \text { 1S } \\ \text { Slivka, John } & \text { 1S } \\ \text { Jamison, Benton } & 2 \mathrm{P} \\ \text { Orey, Steven } & 1 \mathrm{P} \\ \text { Jain, N. C. } & 2 \mathrm{P}\end{array}$

60F99 None of the above, but in this section

Das, Minaketan

$1 \mathrm{P}$ 
[Dec.

\section{Gxx Stochastic processes}

$60 \mathrm{G} 05$ Foundations of stochastic processes

Johnson, Dudley Paul

60G10 Stationary processes

Cambanis, Stamatis

60G15 Gaussian processes

Weissner, Edward W.

Leadbetter, $M$. R.

Strait, Peggy Tang

Jamison, Benton

Orey, Steven

Jain, N. C.

Kuelbs, J. D.

Cambanis, Stamatis

Jain, N. C.

60G17 Sample path properties

Greenwood, Priscilla E.

Weissner, Edward W.

Leadbetter, M. R.

Orey, Steven

Jamison, Benton

Jain, N. C.

Kuelbs, J. D.

Cambanis, Stamatis

60G20 Generalized stochastic processes

Jain, N. C.

Jain, N. C

$1 \mathrm{P}$
$2 \mathrm{P}$

60G25 Prediction theory [See also 62M10.]

$$
\begin{aligned}
& \text { Salehi, Habib } \\
& \text { Scheinberg, Stephen }
\end{aligned}
$$

$2 \mathrm{P}$
$1 \mathrm{~S}$

60G30 Continuity and singularity of induced measures

$$
\begin{aligned}
& \text { Jamison, Benton } \\
& \text { Orey, Steven }
\end{aligned}
$$

Jain, N. C.

60G35 Applications (signal detection, filtering, etc.) [See also 94A05.]

60G40 Stopping times

60G45 Martingale theory

Uhl, J. J., Jr.

Loynes, R. M.

Hebert, D. J., Jr.

Uhl, J. J., Jr.

Brown, B. M

60G50 Sums of independent random variables

Pinsky, Mark A.

Heyde, C. C.

Andersèn, G. R.

Loynes, R. M.

Brown, B. M

Joffe, A. D.

60G99 None of the above, but in this section

60Hxx Stochastic analysis

$60 \mathrm{H} 05$ Stochastic integrals

$\begin{array}{ll}\text { Strait, Peggy Tang } & \text { 2P } \\ \text { Rosencrans, S. I. } & \text { IP }\end{array}$

$60 \mathrm{H} 10$ Stochastic ordinary differential equations [See also 34F05.]

$60 \mathrm{H} 15$ Stochastic partial differential equations

$60 \mathrm{H} 20$ Stochastic integral equations

$60 \mathrm{H} 99$ None of the above, but in this section

60Jxx Markov processes

60J05 Markov processes with discrete parameter

$$
\text { Dubuc, Serge }
$$

$1 \mathbf{P}$

60J10 Markov chains

Ornstein, Donald S.

Yeh, R. Z.

Huff, B. W.

2P

iS

60J15 Random walk

60J20 Applications of discrete Markov processes (social mobility, learning theory, industrial processes, etc.)

$60 \mathrm{~J} 25$ Markov processes with continuous parameter

Sentilles, F. Dennis

$1 \mathrm{P}$

$60 \mathrm{~J} 30$ Processes with independent increments

Strait, Peggy Tang

60J35 Transition functions, generators and resolvents [See also 47D05.]

Johnson, Dudley Paul

$1 \mathbf{P}$

$60 \mathrm{~J} 40$ Hunt processes

60J45 Probabilistic potential theory [See also $31-\mathrm{XX}$.]

Dubuc, Serge

1S
1P

Hebert, D. J., Jr.

$60 J 50$ Boundary theory

$60 J 55$ Local time and additive functionals

$60 \mathrm{~J} 60$ Diffusion processes [See also 28A65.]

60J65 Brownian motion

Greenwood, Priscilla E.

Strait, Peggy Tang

Strait, Peggy Tang

Loynes, R. M.
$1 P$
$1 P$
$2 P$
$1 P$ 
Stackelberg, Olaf $P$

Rosencrans, S. I.

$60 J 70$ Applications of diffusion theory (population genetics, absorption problems, etc.)

$60 J 75$ Jump processes

$60 \mathrm{~J} 80$ Branching processes

(Galton-Watson, birth-and-death, etc.)

Dubuc, Serge

Athreya, Krishna Balasundaram 1P

60J85 Applications of branching processes [See also 90Bxx.]

$60 J 99$ None of the above, but in this section

\section{$60 \mathrm{Kxx}$ Special processes}

60K05 Renewal theory

60K10 Applications (reliability, demand theory, etc.)

60K15 Markov renewal processes, semi-Markov processes

60K20 Applications of Markov renewal processes (reliability, queueing networks, etc.)

60K25 Queueing theory

60K30 Applications (congestion, allocation, storage, traffic, etc.) [See also 60J80.]

60K35 Interacting random processes, statistical mechanics type models

$60 \mathrm{~K} 99$ None of the above, but in this section

\section{2-XX STATISTICS}

62-00 Difficult to classify at the second level (must also be assigned at least one other classification number in this section)

62-01 Elementary exposition (collegiate level)

62-02 Advanced exposition (research surveys, etc.)

62-03 Historical

62-04 Explicit machine computation and programs (not the theory of computation or programming)

\section{Axx Foundations}

62A05 Invariance and group considerations

62A10 The likelihood approach
62A15 The Bayesian approach

62A99 None of the above, but in this section

\section{Bxx Sufficiency}

62B05 Sufficient statistics and fields

62B10 Statistical information theory

62B15 Comparison of experiments

62B20 Measure-theoretic results, etc.

62B99 None of the above, but in this section

62Cxx Decision theory (For game theory, see 90Dxx.

$62 \mathrm{C} 05$ General considerations

$62 \mathrm{C07}$ Complete class results

62C10 Bayesian problems; characterization of Bayes procedures

62C15 Admissibility

62C25 Compound decision problems

$62 C 99$ None of the above, but in this section

62D05 Sampling theory, sample surveys

\section{Exx Distribution theory}

62E10 Characterization and structure theory

\begin{tabular}{ll} 
Jamison, Benton & 2S \\
Orey, Steven & 1S \\
Tata, Mahabanoo N. & 1P \\
Staudte, Robert G., Jr. & 1P \\
Athreya, Krishna Balasundaram & $1 \mathrm{P}$ \\
15 Distribution of statistics & \\
Loynes, R. M. & $1 \mathrm{~S}$ \\
\hline 0 Asymptotic theory & \\
Andersen, G. R. & $1 \mathrm{~S}$ \\
Severo, Norman C. & $1 \mathrm{P}$ \\
Slivka, John & $1 \mathrm{P}$ \\
Loynes, R. M. & $1 \mathrm{P}$ \\
Das, Minaketan & $1 \mathrm{P}$
\end{tabular}

62E25 Monte Carlo studies

62 E99 None of the above, but in this section

62Fxx Parametric inference

62F05 Hypothesis testing

62F07 Ranking and selection

62F10 Estimation 


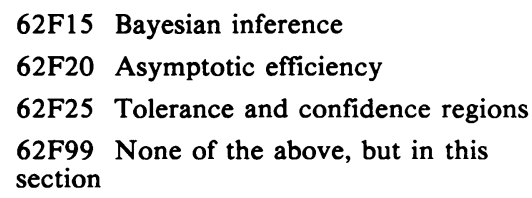

\section{Hxx Multivariate analysis}

62H05 Characterization and structure theory

$62 \mathrm{H} 10$ Distribution of statistics

62H15 Hypothesis testing

$62 \mathrm{H} 20$ Correlation analysis, canonical correlation

$62 \mathrm{H} 25$ Factor analysis and principal components

$62 \mathrm{H} 30$ Classification and discrimination $62 \mathrm{H} 99$ None of the above, but in this section

\section{Jxx Linear inference}

62J05 Regression analysis

$62 \mathrm{~J} 10$ Analysis of variance and covariance

62J15 Paired and multiple comparisons

$6.2 \mathrm{~J} 99$ None of the above, but in this section

62Kxx Experimental design \{See also 05Bxx.\}

62K05 Optimal designs

62K10 Block designs

$62 \mathrm{~K} 15$ Factorial designs

$62 \mathrm{~K} 99$ None of the above, but in this section

\section{Lxx Sequential methods}

62L05 Sequential design

62L10 Sequential analysis

62L12 Sequential estimation

62L15 Optimal stopping [See also 60G40.]

62L20 Stochastic approximation

62L99 None of the above, but in this section

$62 \mathrm{Mxx}$ Inference from stochastic processes

62M05 Markov processes

$62 \mathrm{M} 10$ Time series, auto-correlation, regression, etc.

Kuelbs, J. D.

62M15 Spectral analysis of time series Kuelbs, J. D. Jain, N. C. is

62M20 Prediction [See also 60G25.]

$62 \mathrm{M} 99$ None of the above, but in this section

\section{$62 \mathrm{Nxx}$ Engineering statistics}

62N05 Reliability and life testing 62N10 Quality control [See also 90Bxx.]

62 N 15 Stochastic differential and difference equations [See also 34F05, $60 \mathrm{H} 10,93 \mathrm{Exx}$.]

62 N99 None of the above, but in this section

62Pxx Applications \{See also $82-\mathrm{XX}, 90-\mathrm{XX}, 92-\mathrm{XX}$.

62P05 Actuarial mathematics

62P10 Biometrics

62P15 Psychometrics

62P20 Econometrics

62P25 Sociometrics

62 P99 None of the above, but in this section

\section{Q05 Statistical tables}

\section{5-XX NUMERICAL} ANALYSIS

65-00 Difficult to classify at the second level (must also be assigned at least one other classification number in this section) 
65-01 Elementary exposition (collegiate level)

65-02 Advanced exposition (research surveys, etc.)

65-03 Historical

\section{A05 Tables}

65Bxx Acceleration of convergence

65B05 Extrapolation to the limit, deferred corrections

65B10 Summation of series

65B15 Euler-MacLaurin formula

65B20 Poisson formula, etc.

65B99 None of the above, but in this section

65Cxx Numerical simulation \{See also 68A55.

65C05 Monte Carlo methods

65C10 Random number generation

$65 C 99$ None of the above, but in this section

65Dxx Numerical approximation \{Primarily algorithms; for theory, see $41-X X$.

65D05 Interpolation

65D10 Smoothing, curve fitting

65D15 Algorithms for functional approximation

65D20 Computation of special functions, construction of tables

65D25 Numerical differentiation

65D30 Numerical integration, quadrature, cubature, etc.

$$
\text { Yeh, J. }
$$

65D99 None of the above, but in this section

65E05 Numerical methods in complex analysis (potential theory, etc.) (For numerical methods in conformal mapping, see 30A28.

65Fxx Numerical linear algebra 65F05 Direct methods for linear systems and matrix inversion

$65 \mathrm{~F} 10$ Iterative methods for linear systems [See also 65N20.]

$\begin{array}{ll}\text { Block, Henry David } & \text { IP } \\ \text { Levin, Simon A. } & \text { IP }\end{array}$

65F15 Eigenvalues, eigenvectors

65F20 Overdetermined systems, pseudoinverses

65F25 Orthogonalization

65F30 Other matrix algorithms

65F35 Matrix norms, conditioning, scaling [See also 15A12, 15A60.]

65 F99 None of the above, but in this section

\section{G05 Roundoff error}

$65 \mathrm{Hxx}$ Nonlinear algebraic or transcendental equations

65H05 Single equations

$65 \mathrm{H} 10$ Systems of equations

65J05 Numerical analysis in abstract spaces

65K05 Mathematical programming and optimization techniques \{Computational aspects; for algorithms and theory, see 49Exx and 90Cxx.

Block, Henry David 1S

Levin, Simon A.

65Lxx Ordinary differential equations

$65 \mathrm{~L} 05$ Initial value problems

$65 \mathrm{~L} 10$ Boundary value problems

65L15 Eigenvalue problems

65 L99 None of the above, but in this section

65Mxx Partial differential equations, initial value problems

65M05 Derivation of finite difference approximations

$65 \mathrm{M} 10$ Stability and convergence of difference methods

65M15 Error bounds

65M20 Method of lines

65M25 Method of characteristics 
65M30 Improperly posed problems

$65 \mathrm{M} 99$ None of the above, but in this section
68-03 Historical

68A05 Programming theory

68A10 Algorithms [See also 02E10.]

65Nxx Partial differential equations, 68A15 Symbolic computation boundary value problems

65N05 Derivation of finite difference approximations

$65 \mathrm{~N} 10$ Convergence of difference methods

65 N 15 Error bounds

65 N20 Solution of difference equations [See also 65F10.]

$65 \mathrm{~N} 25$ Eigenvalue problems

65N30 Rayleigh-Ritz and Galerkin methods

65 N35 Collocation and related methods

65 N99 None of the above, but in this section

68A20 Computational complexity and efficiency

68A25 Automata theory [See also 02F10, 18B20, 94A30, 94A35.]

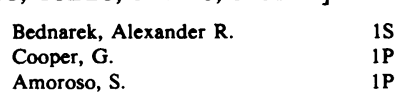

68A30 Linguistics, formal languages

68A35 Adaptive systems

68A40 Theorem proving

68A45 Artificial intelligence, pattern recognition

68A50 Information retrieval

68A55 Simulation [See also 65Cxx.]

65P05 Partial differential equations, miscellaneous problems

65Q05 Difference and functional equations, recurrence relations

Marzec, R. P.

Franks, R. L.

65R05 Integral and integro-differential equations

$65 \mathrm{~S} 05$ Graphical methods

65T05 Harmonic analysis and synthesis

68-XX COMPUTER

SCIENCE (For papers involving machine computations and programs in a specific mathematical area, see section -04 in that area $\}$

68-00 Difficult to classify at the second level (must also be assigned at least one other classification number in this section)

68-01 Elementary exposition (collegiate level)

68-02 Advanced exposition (research surveys, etc.)

PARTICLES AND SYSTEMS
PAC \{For relativistic mechanics, see $83 \mathrm{~A} 05$ and $83 \mathrm{Cl} 10$; for statistical mechanics, see $82-\mathrm{XX}$.

$70-00$ Difficult to classify at the second level (must also be assigned at least one other classification number in this section)

70-01 Elementary exposition (collegiate level)

70-02 Advanced exposition (research surveys, etc.)

70-03 Historical

70-04 Explicit machine computation and programs (not the theory of computation or programming)

\section{A05 Axiomatics}

70Bxx Kinematics

70B05 Kinematics of a particle

70B10 Kinematics of a rigid body

70B15 Mechanisms and linkages

70 B99 None of the above, but in this section

70Cxx Statics

$70 \mathrm{C05}$ Forces, fields 
$70 \mathrm{C} 10$ Potential energy

$70 C 99$ None of the above, but in this section

70Dxx Dynamics of a particle $\{$ See also $70 \mathrm{Hxx}$.

70D05 Newtonian dynamics

70D10 Lagrangian dynamics

70D99 None of the above, but in this section

\section{Exx Dynamics of a rigid body}

70E05 Motion of the gyroscope

$70 \mathrm{E} 10$ Motion of projectiles and rockets

$70 \mathrm{E} 15$ Motion of a general rigid body

70E20 Perturbation methods for Euler's equations

70E25 Poinsot method

70 E99 None of the above, but in this section

70Fxx Dynamics of a system of particles

70F05 Two-body problemn-body problem

70 F15 Celestial mechanics

70F20 Holonomic systems

70F25 Nonholonomic systems

70F30 Impulsive motion

70F35 Collisions

70F99 None of the above, but in this section

70Gxx General representations of dynamical systems

70 G05 Riemannian geometry, tensorial methods [See also 53A45, 53A50, 53B20.]

70G10 Generalized coordinates

70 G15 Space of events

70G20 Impulse-energy space

70 G25 Configuration space

70 G30 State space

70G35 Phase space

70G99 None of the above, but in this section
70Hxx Hamiltonian mechanics

70H05 Hamilton's equations

$70 \mathrm{H} 10$ Liouville's theorem

$70 \mathrm{H} 15$ Canonical transformations

$70 \mathrm{H} 20$ Hamilton-Jacobi equations

$70 \mathrm{H} 25$ Variational methods

$70 \mathrm{H} 99$ None of the above, but in this section

70Jxx Linear vibration theory

70J05 Finite degree of freedom systems

$70 \mathrm{~J} 10$ Normal modes of vibrations

$70 \mathrm{~J} 15$ Conservative systems

70J20 Nonconservative systems

70J25 Stability of oscillatory motions

$70 J 99$ None of the above, but in this section

$70 \mathrm{Kxx}$ Nonlinear oscillations

70K05 Phase plane analysis

70K10 Limit cycles

$70 \mathrm{~K} 15$ Ljapunov theorems

70K20 Stability

70K99 None of the above, but in this section

70L05 Random vibrations \{ See also 93Exx.\}

70Mxx Orbital mechanics

70M05 Satellite problems

70M10 Orbital stability

70M99 None of the above, but in this section

70N05 Exterior ballistics

70P05 Variable mass, rockets

\section{3-XX MECHANICS OF SOLIDS}

73-00 Difficult to classify at the second level (must also be assigned at least one other classification number in this section)

73-01 Elementary exposition (collegiate level)

73-02 Advanced exposition (research 
surveys, etc.)

73-03 Historical

73-04 Explicit machine computation and programs (not the theory of computation or programming)

\section{A05 Axiomatics}

73Bxx Continuum mechanics (For fluids, see 76Axx.

73B05 Constitutive equations

$73 \mathrm{~B} 10$ Isotropic functionals

73B15 Rotational groups [See also 22Exx.]

73B20 Simple materials

73B25 Multipolar stress theory

73B30 Thermodynamics of solids [For gases and fluids, see $80-\mathrm{XX}$.]

73B99 None of the above, but in this section

73Cxx Linear elasticity (For the biharmonic equation, see $31 \mathrm{~B} 30$; for acoustics, see 76Q05.\}

$73 \mathrm{CO5}$ Stress functions

$73 \mathrm{C} 10$ Saint-Venant's principle

$73 \mathrm{C} 15$ Uniqueness theorems

73C20 Strain energy methods

73C25 Thermal stress problems

73C30 Anisotropic bodies

73C35 Mixed boundary value problems [See also 45F05.]

73 C99 None of the above, but in this section

73Dxx Wave propagation in solids

73D05 Impact and explosion problems [See also 76L05.]

73D10 Integral transforms

73D15 Dilatational and shear waves

73D20 Surface waves

73D25 Wave diffraction and dispersion

73D99 None of the above, but in this section

\section{Exx Plasticity}

73E05 Yield criteria and flow rules
73E10 Method of successive

approximations

73E15 Slip-line theory

73E20 Limit analysis

73E25 Creep

73E99 None of the above, but in this section

\section{Fxx Viscoelasticity}

73F05 Creep and relaxation functionals

73F10 Correspondence principle

73F15 Time-dependent boundary value problems

73F20 Aging of materials

73F25 Environmental-dependent materials

73F99 None of the above, but in this section

73Gxx Finite deformations

73G05 Finite elasticity

73G10 Strain energy functions

73 G15 Finite viscoelasticity

73G20 Metal forming problems

73 G99 None of the above, but in this section

73Hxx Elastic stability

73H05 Buckling

73H10 Dynamic stability

73H99 None of the above, but in this section

\section{Jxx Aeroelasticity}

73J05 Interaction of aerodynamics and elasticity

73J10 Vibrations, flutter

73J15 Divergence

73J99 None of the above, but in this section

73Kxx Structural mechanics

73K05 Beams, columns

73K10 Plates

73K15 Membranes, shells

73K20 Composite structures

73K25 Finite element methods 
73K99 None of the above, but in this section

\section{Lxx Theory of shells}

73L05 Noneuclidean geometry, tensorial methods [See also 53A45.]

73L10 Anisotropic shells

73L15 Shell dynamics

73L99 None of the above, but in this section

\section{Mxx Fractural mechanics}

73M05 Brittle fracture, cracks

73M10 Fatigue

73M15 Ductile fracture

73M20 Material instability

73 M99 None of the above, but in this section
76-02 Advanced exposition (research surveys, etc.)

76-03 Historical

76-04 Explicit machine computation and programs (not the theory of computation or programming)

76Axx Constitutive equations (See also 35L65.

76A05 Non-Newtonian fluids

76A10 Viscoelastic fluids

76A99 None of the above, but in this section

76Bxx Incompressible, inviscid fluids, potential theory

76B05 Airfoil theory

76B10 Jets and cavities, free-streamline theory, water-entry problems, hydrofoil

\section{$73 N x x$ Geophysical solid mechanics theory}

\{See also 86-XX.

73N05 Global dynamics

73 N10 Earthquake problems

73N99 None of the above, but in this section

73Pxx Biomechanics of solids

73P05 Mathematical models of biological materials

73P10 Mechanics response

73P99 None of the above, but in this section

\section{Q05 Soil mechanics}

73R05 Electromagnetic elasticity

73Sxx Micromechanics of solids

73S05 Dislocation theory

73S99 Other micromechanics

76-XX FLUID MECHANICS

\{For general continuum mechanics, see $73 B x x$.

76-00 Difficult to classify at the second level (must also be assigned at least one other classification number in this section)

76-01 Elementary exposition (collegiate level)
76B15 Water waves, gravity waves; dispersion and diffraction, nonlinear interaction

76B20 Ship waves

76B25 Solitary and cnoidal waves

76B99 None of the above, but in this section

76Cxx Incompressible inviscid

fluids, vorticity flows

$76 \mathrm{C05}$ Vorticity flows

$76 \mathrm{C} 10$ Internal waves

$76 \mathrm{C} 15$ Atmospheric waves

76C20 Rossby waves

$76 \mathrm{C} 99$ None of the above, but in this section

76Dxx Incompressible viscous fluids

76D05 Navier-Stokes equations [See also 35Q10.]

76D10 Boundary-layer theory

76D15 Boundary-layer separation and reattachment

76D20 Higher-order effects in boundary layers

76D25 Wakes and jets

76D30 Singular perturbation problems

76D99 None of the above, but in this 
[Dec.

section

76Exx Hydrodynamic stability

76E05 Stability of parallel flows

76E10 Inertial instability

76E20 Instability of geophysical and astrophysical flows

76E25 Magnetohydrodynamic and electrohydrodynamic instabilities

76E30 Nonlinear effects

76E99 None of the above, but in this section

76F05 Turbulence (See also $60 \mathrm{Gxx}$, $60 \mathrm{Jxx}$.

\section{Gxx Subsonic flows}

76G05 Hodograph methods

76G10 Kármán-Tsien approximation

76 G15 Iterative methods

76G20 Free-streamline theory

$76 \mathrm{G} 99$ None of the above, but in this section

76H05 Transonic flows, limit lines

76Jxx Supersonic flows

76J05 Hodograph methods

$76 \mathrm{~J} 10$ Method of characteristics

76J99 None of the above, but in this section

76K05 Hypersonic flows
76E15 Convective instability

76Rxx Diffusion and convection

\{See also 60J60.

76R05 Forced convection

76R 10 Free convection

76R99 None of the above, but in this section

76S05 Flows in porous media

76T05 Two-phase and multiphase

flows

76U05 Rotating fluids

76V05 Stratified fluids

76W05 Magnetohydrodynamics and electrohydrodynamics

76X05 Ionized gas flow in electromagnetic fields

76Y05 Quantum hydrodynamics and relativistic hydrodynamics \{See also 83C55, 85A30.

76Zxx Biological fluid mechanics

76Z05 Physiological flows

$76 \mathrm{Z} 10$ Biopropulsion in water and in air

$76 Z 99$ None of the above, but in this section

\section{8-XX OPTICS, ELECTROMAGNETIC THEORY}

76L05 Shock waves and blast waves 78-00 Difficult to classify at the \{See also 73D05.\}

76M05 Nonhomentropic flows of compressible fluids

76N05 Boundary-layer theory of compressible fluids

76P05 Rarefied gas flows, Boltzmann equation (See also 82A05.\}

76Q05 Hydrodynamic sound, acoustics second level (must also be assigned at least one other classification number in this section)

78-01 Elementary exposition (collegiate level)

78-02 Advanced exposition (research surveys, etc.)

78-03 Historical

78-04 Explicit machine computation and programs (not the theory of computation or programming)

78A05 Geometric optics

78A10 Physical optics

78A15 Electron optics 
78A20 Space charge waves

78A25 Electromagnetic theory

78A30 Electro- and magnetostatics

78A35 Motion of charged particles

78A40 Waves and radiation

78A45 Diffraction, scattering [For WKB methods, see also 34E20.]

78A50 Antennas, wave-guides

78A55 Technical applications

78A57 Mathematically heuristic optics and electromagnetic theory (must also be assigned at least one other classification number in this section)

\section{0-XX CLASSICAL} THERMODYNAMICS, HEAT TRANSFER \{For thermodynamics of solids, see 73B30. \}

80-00 Difficult to classify at the second level (must also be assigned at least one other classification number in this section)

80-01 Elementary exposition (collegiate level)

80-02 Advanced exposition (research surveys, etc.)

80-03 Historical

80-04 Explicit machine computation and programs (not the theory of computation or programming)

80A05 Foundations

80A10 Classical thermodynamics

80A15 Thermodynamics of mixtures

80A20 Heat and mass transfer

80A25 Combustion, interior ballistics

80A30 Chemical kinetics

80A35 Mathematically heuristic classical thermodynamics (must also be assigned at least one other classification number in this section)

\section{1-XX QUANTUM MECHANICS}

81-00 Difficult to classify at the second level (must also be assigned at least one other classification number in this section)
81-01 Elementary exposition (collegiate level)

81-02 Advanced exposition (research surveys, etc.)

81-03 Historical

81-04 Explicit machine computation and programs (not the theory of computation or programming)

81A06 Relativistic theory

81A09 Selfadjoint operator theory in quantum mechanics, essential selfadjointness of the Hamiltonian

81A10 Perturbation theory

81 A12 Logical foundations of quantum mechanics

Morash, Ronald P.

81A15 Feynman integrals and graphs, applications of algebraic topology and algebraic geometry to these problems

81A17 Axiomatic quantum field theory; operator algebras

81A18 Constructive quantum field theory; models of quantum fields

81A19 Renormalization theory

81A20 Commutation relations

Prosser, Reese T.

81A24 Bethe-Salpeter equation

81A27 Current algebra

81A30 Broken symmetries

81A33 Covariant wave equations

81A36 Strong interaction

81A39 Electromagnetic interaction

81A42 Weak interaction

81A45 Potential scattering theory [For WKB methods, see also 34E20.]

$81 \mathrm{~A} 48 \mathrm{~S}$-matrix theory and other scattering theory

81A51 Dispersion theory

81A54 Applications of group theory to elementary particles

81A57 Other elementary particle theory 81 A60 Applications of group theory to nuclear physics

81A63 Other nuclear physics

81A66 Applications of group theory to atomic physics

81A69 Other atomic physics 
81A72 Applications of group theory to molecular physics

81A75 Other molecular physics

81 A78 General group representation motivated by physics but not covered by 81A54, 81A60, 81A66, 81A72, representations of concrete classical groups such as $\operatorname{SL}(n, C), U(p, q)$, etc.

Vasquez, Alphonse T.

Lazarov, Conno

81A81 Quantum mechanics of many-body systems

81A84 Superconductivity and superfluidity

81A87 Mathematically heuristic quantum mechanics (must also be assigned at least one other classification number in this section)

\section{2-XX STATISTICAL PHYSICS, STRUCTURE OF MATTER}

$82-00$ Difficult to classify at the second level (must also be assigned at least one other classification number in this section)

82-01 Elementary exposition (collegiate level)

82-02 Advanced exposition (research surveys, etc.)

82-03 Historical

82-04 Explicit machine computation and programs (not the theory of computation or programming)

82A05 Mathematical general statistical mechanics

82A15 Mathematical quantum statistical mechanics

82A25 Phase transitions

82A30 Statistical thermodynamics [See also 80 - XX.]

82A35 Irreversible thermodynamics

$82 \mathrm{~A} 40$ Kinetic theory of gases

$82 \mathrm{~A} 45$ Plasma

82A50 Liquids

82A55 Solids

82A60 Crystals

82A65 Metals

82A70 Transport processes [See also
85A25.]

82A75 Nuclear reactor theory

82A77 Mathematically heuristic statistical physics (must also be assigned at least one other classification number in this section)

\section{3-XX RELATIVITY}

83-00 Difficult to classify at the second level (must also be assigned at least one other classification number in this section)

83-01 Elementary exposition (collegiate level)

83-02 Advanced exposition (research surveys, etc.)

83-03 Historical

83-04 Explicit machine computation and programs (not the theory of computation or programming)

\section{A05 Special relativity \\ 83B05 Observational and experimental questions}

83Cxx General relativity

83C05 Einstein's equation

$83 \mathrm{C} 10$ Equations of motion

$83 \mathrm{C} 15$ Closed form solutions

$83 \mathrm{C} 20$ Classes of solutions

83C25 Approximation procedures, weak fields

83C30 Asymptotic procedures (radiation, news functions, etc.)

83C35 Gravitational waves

$83 \mathrm{C} 40$ Groups of motions, invariance groups, observation laws, etc.

83C45 Quantization of the gravitational field

83C50 Electromagnetic fields

83C55 Hydrodynamics [See also 76Y05.]

$83 C 99$ None of the above, but in this section

83D05 Relativistic gravitational theories other than Einstein's

83Exx Unified field theories

83E05 Geometrodynamics 
83E10 Asymmetric field theories

$83 \mathrm{E} 15$ Five- and higher-dimensional theories

83E99 None of the above, but in this section

\section{F05 Cosmology}

\section{5-XX ASTRONOMY AND} ASTROPHYSICS (For celestial mechanics, see 70F15.

85-00 Difficult to classify at the second level (must also be assigned at least one other classification number in this section)

85-01 Elementary exposition (collegiate level)

85-02 Advanced exposition (research surveys, etc.)

85-03 Historical

85-04 Explicit machine computation and programs (not the theory of computation or programming)

85A05 Galactic and stellar dynamics

85A10 Astronautics

85A15 Stellar structure

85A20 Stellar atmospheres

85A25 Radiative transfer

85A30 Hydrodynamic and hydromagnetic problems [See also 76Y05.]

85A35 Statistical astronomy

85A40 Cosmology [For relativistic cosmology, see 83F05.]

85A45 Radio astronomy

86-XX GEOPHYSICS \{See also $73 \mathrm{Nxx}, 76 \mathrm{U} 05,76 \mathrm{~V} 05$.

86-00 Difficult to classify at the second level (must also be assigned at least one other classification number in this section)

86-01 Elementary exposition (collegiate level)

86-02 Advanced exposition (research surveys, etc.)

$86-03$ Historical

86-04 Explicit machine computation and programs (not the theory of computation or programming)

86A05 Hydrology, hydrography, oceanography

86A10 Meteorology

86A15 Seismology

86A20 Potentials, prospecting

86A25 Geo-electricity and geomagnetism

86A30 Geodesy, mapping problems

86A35 Atmospheric physics

90-XX ECONOMICS, OPERATIONS RESEARCH, PROGRAMMING, GAMES

90-00 Difficult to classify at the second level (must also be assigned at least one other classification number in this section)

90-01 Elementary exposition (collegiate level)

90-02 Advanced exposition (research surveys, etc.)

90-03 Historical

90-04 Explicit machine computation and programs (not the theory of computation or programming)

90Axx Mathematical economics \{For econometrics, see 62P20.\} 90A05 Decision theory [See also 62Cxx.]

90A10 Utility theory

90A15 Economic models

90A20 Economic time series analysis [See also 62M10.]

90A99 None of the above, but in this section

90Bxx Operations research and management science

90B05 Logistics, inventory, storage

90B10 Flows in networks, deterministic

90B15 Flows in networks, probabilistic

90B20 Highway traffic

90B25 Reliability and maintenance [See also $60 \mathrm{~K} 20,62 \mathrm{~N} 05$.]

90B30 Production theory 
90B35 Scheduling theory

90B40 Search theory

90B99 None of the above, but in this section

90Cxx Mathematical programming (For dynamic programming, see 49Cxx; for papers emphasizing calculus of variations or involving abstract spaces, see 49Dxx.\}

$90 \mathrm{C05}$ Linear programming

$90 \mathrm{C} 10$ Integer programming

90C15 Stochastic programming

90C20 Quadratic programming

90C25 Convex programming

90C30 Nonlinear programming

90C35 Network programming, programming in networks

90C40 Markov programming

90C45 Markov renewal programming

90C50 Applications of mathematical programming

90C99 None of the above, but in this section

\section{Dxx Game theory}

90D05 2-person zero-sum games 90D10 n-person games, noncooperative 90D12 n-person games, cooperative, solution concepts

$$
\text { Parthasarathy, T. }
$$

$1 \mathrm{P}$

90D13 Games for a continuum of players

90D15 Multistage games, stochastic [See also 93E05.]

90D20 Multistage games, recursive

90D25 Differential games

90D30 Utility theory [See also 90A10.]

90D35 Decision theory [See also 62Cxx, 90A05.]

90D40 Game theory models [See also 65Cxx, 68A55.]

90D45 Applications of game theory

90D99 None of the above, but in this section

\section{2-XX BIOLOGY AND}

\section{BEHAVIORAL SCIENCES}

92-00 Difficult to classify at the second level (must also be assigned at least one other classification number in this section)

92-01 Elementary exposition (collegiate level)

92-02 Advanced exposition (research surveys, etc.)

92-03 Historical

92-04 Explicit machine computation and programs (not the theory of computation or programming)

92A05 Biology [See also 73Pxx, 76Zxx.]

92A10 Genetics

92A15 Population dynamics, epidemiology

92A20 Sociology

92A25 Psychology

\section{3-XX SYSTEMS, CONTROL}

(For optimal control (nonstochastic), see $49-\mathrm{XX}$. \}

93-00 Difficult to classify at the second level (must also be assigned at least one other classification number in this section)

93-01 Elementary exposition (collegiate level)

93-02 Advanced exposition (research surveys, etc.)

93-03 Historical

93-04 Explicit machine computation and programs (not the theory of computation or programming)

\section{Axx General}

93A05 Axiomatic system theory

93A10 General systems

93A15 Large scale systems (approximation techniques)

93A99 None of the above, but in this section

93Bxx Controllability, observability, and system structure

93B05 Controllability, observability, definitions and criteria

93B10 Canonical structure 
93B15 Realizability of systems from input-output data

93B20 Minimal systems representations 93B25 Algebraic theory of time-invariant systems

93B30 System identification

93B35 Sensitivity

93B99 None of the above, but in this section

93Cxx Control systems, guided systems

93C05 Linear

93C10 Nonlinear

93C15 Systems governed by ordinary differential equations

93C20 Systems governed by partial differential equations

93C22 Systems governed by integral equations

93C25 Systems in abstract spaces

93C30 Systems governed by functional relations other than differential or integral equations

93C40 Adaptive

Block, Henry David

Levin, Simon A.

93C45 Time-invariant

93C50 Time-dependent

93C55 Discrete-time

93C60 Continuous-time

93C99 None of the above, but in this section

\section{Dxx Stability}

93D05 Ljapunov stability

93D10 Popov-type stability of feedback systems

93D15 Stabilization of systems by feedback

93D99 None of the above, but in this section

\section{Exx Stochastic systems and} control

93E05 Stochastic games, stochastic differential games

62A10 The likelihood approach
93E10 Estimation problems (filtering, prediction, data smoothing, system identification)

93E15 Stochastic stability

93E20 Optimal stochastic control (continuous parameter)

93E25 Computational methods

93E99 None of the above, but in this section

94-XX INFORMATION AND COMMUNICATION, CIRCUITS, AUTOMATA For papers in pattern recognition, linguistics and formal languages, see 68A30, 68A45. \}

94-00 Difficult to classify at the second level (must also be assigned at least one other classification number in this section)

94-01 Elementary exposition (collegiate level)

94-02 Advanced exposition (research surveys, etc.)

94-03 Historical

94-04 Explicit machine computation and programs (not the theory of computation or programming)

94A05 Communication theory [See also 60J30.]

94A10 Coding theory

94A15 Information theory [See also 62B10.]

94A20 Circuits, networks; applications of graph theory and Boolean algebra

$$
\text { Rosenfeld, } M \text {. }
$$

94A25 Sequential machines

94A30 Automata, general [See also 02F10, 18B20, 68A25.]

Siromoney, Rani

Bednarek, Alexander $\mathbf{R}$.

Levin, Simon A

Block, Henry David

1P

1S

1S

94A35 Probabilistic automata

Santos, Eugene S.

$1 \mathbf{P}$ 